\title{
Magnetic Multilayer Structures for Spin Torque Nano Oscillator
}

\author{
Linqiang Luo \\ Chenzhou, Hunan, China \\ Bachelor of Science, Hunan Normal University, 2008 \\ Master of Science, Nanjing University, 2011
}

\begin{abstract}
A Dissertation presented to the Graduate Faculty
of the University of Virginia in Candidacy for the Degree of

Doctor of Philosophy

Department of Physics
\end{abstract}

University of Virginia

May, 2017 


\section{CONTENTS}

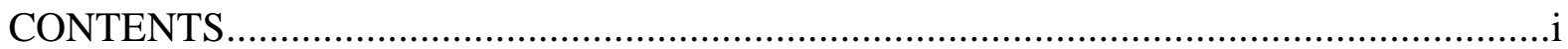

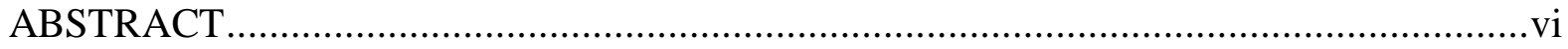

ACKNOWLEDGEMENTS .............................................................................. viii

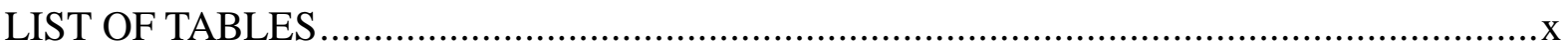

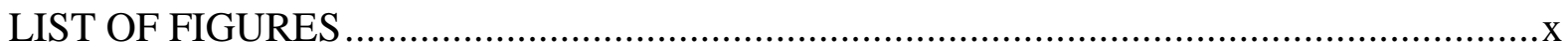

Chapter 1 Introduction ...........................................................................

1.1 An overview of the dissertation .............................................................. 1

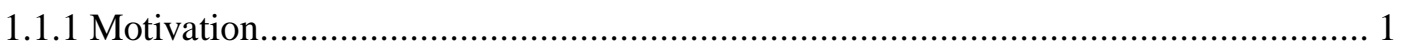

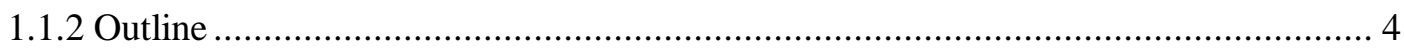

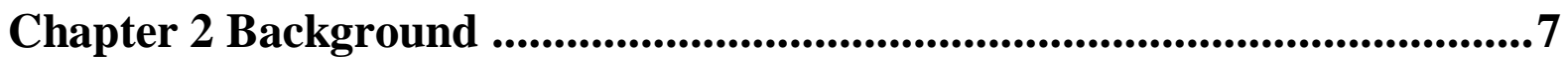

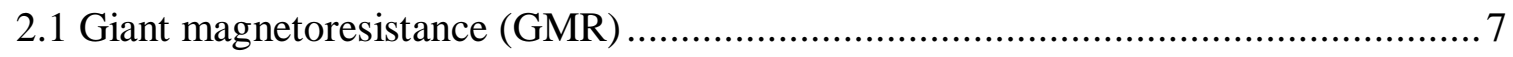

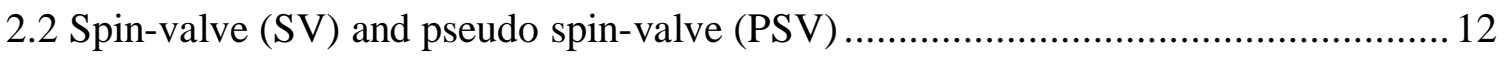

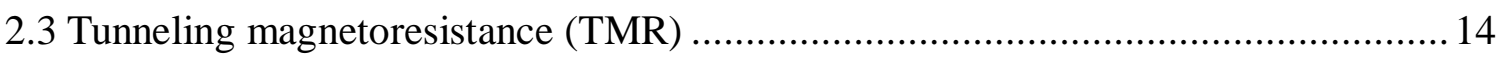

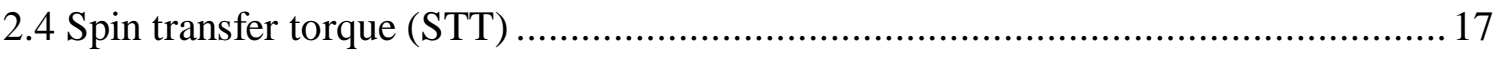

2.4.1 The model for spin transfer-Slonczewski model ......................................... 19

2.4.2 The critical current density $\left(J_{c}\right)$ for magnetization reversal ................................ 23

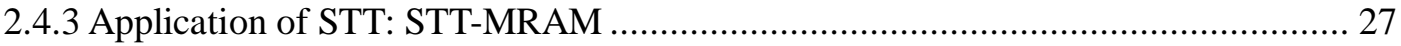




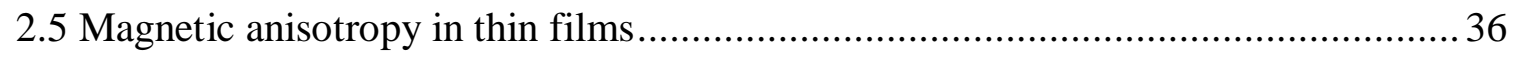

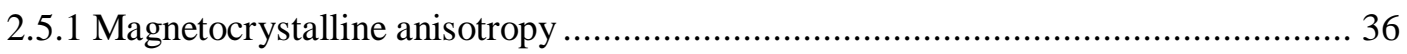

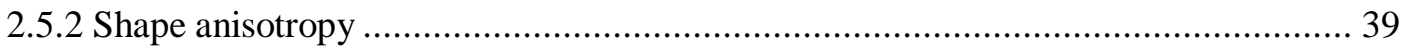

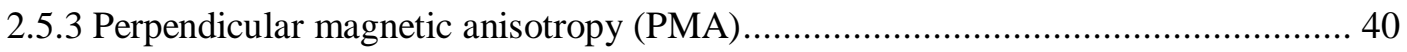

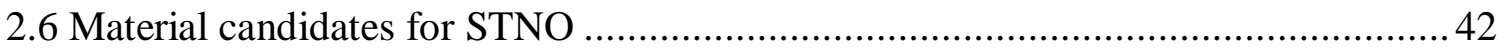

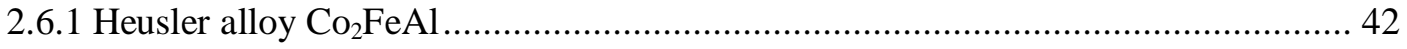

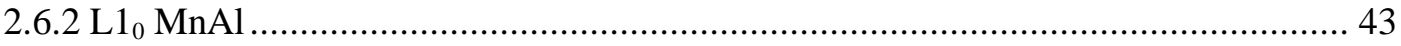

Chapter 3 Experimental techniques...........................................................46

3.1 Multilayer film fabrication by RBTIBD ............................................. 46

3.1.1 Deposition of epitaxy $\mathrm{MnAl}$ on $\mathrm{MgO}(001)$ substrate ........................................... 52

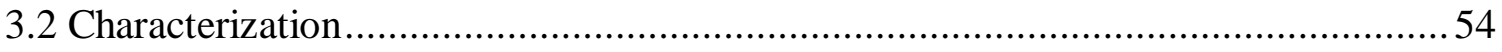

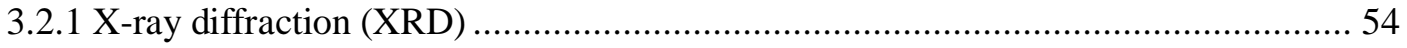

3.2.2 Physics property measurement system (PPMS) …............................................ 55

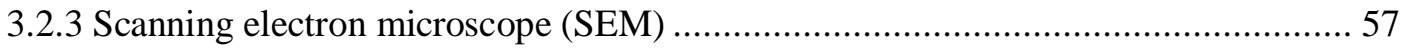

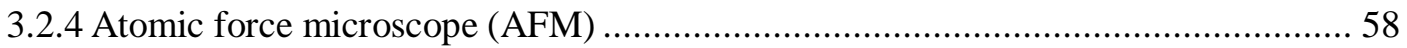

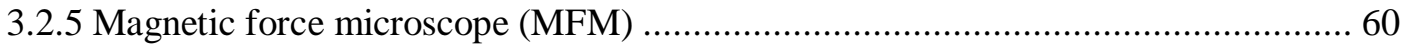

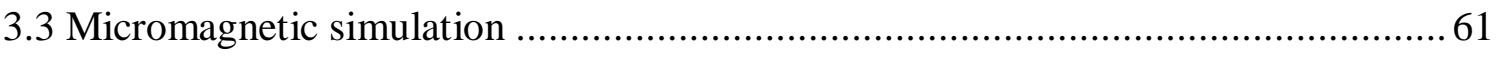

Chapter 4 Magnetic multilayers with $\mathrm{Co}_{2} \mathrm{FeAl}$..............................................63 
4.1 Introduction

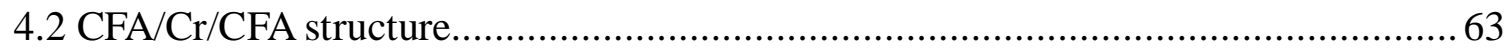

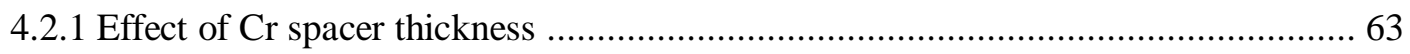

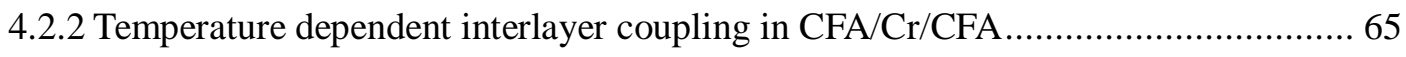

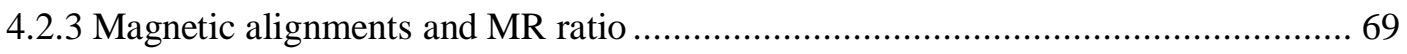

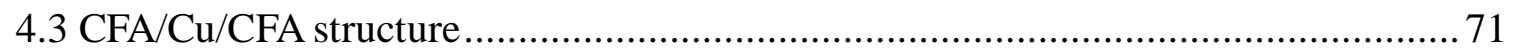

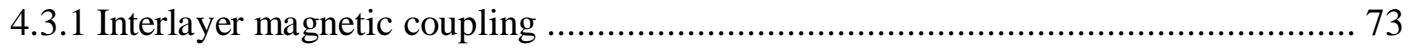

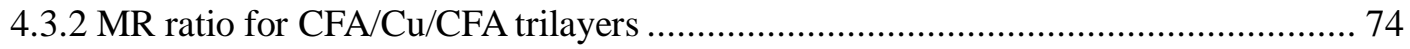

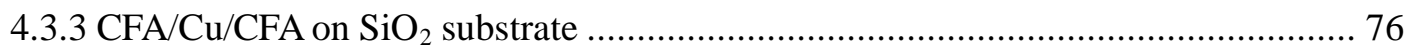

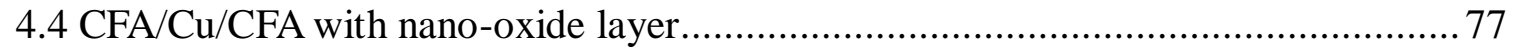

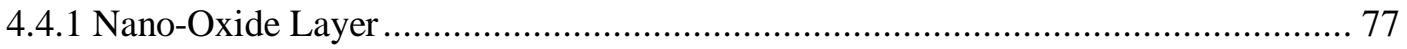

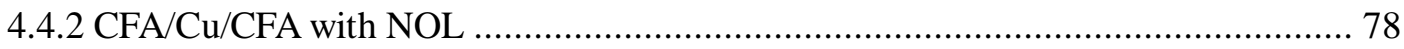

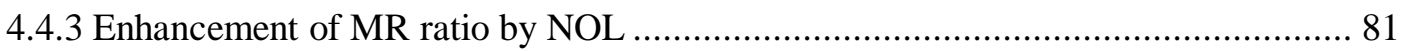

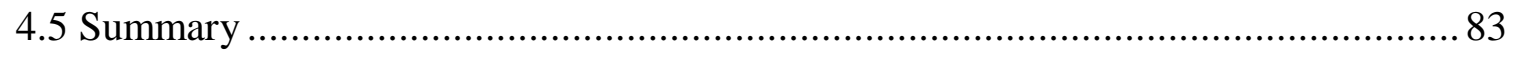

Chapter 5 L1 $1_{0} \mathrm{MnAl}$ and magnetic multilayers based on MnAl ..................85

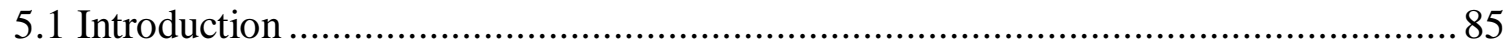

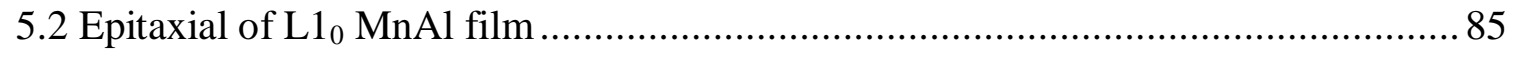

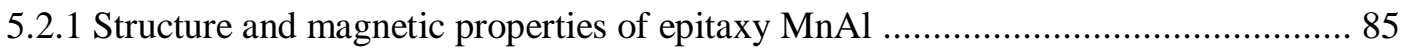

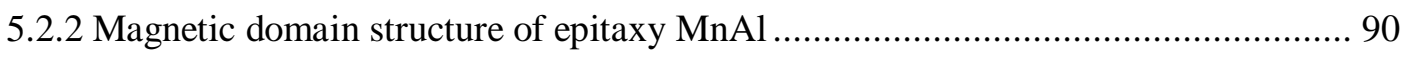

5.3 Magneto-transport and domain wall scattering in epitaxial $\mathrm{L1}_{0} \mathrm{MnAl}$ thin film...... 91 
5.3.1 Current-in-plane patterning

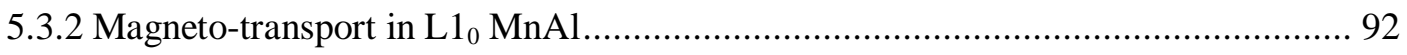

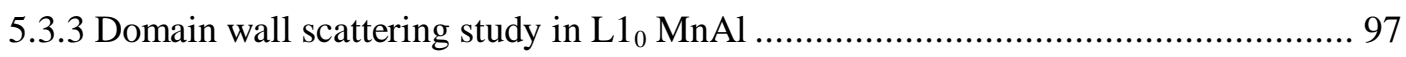

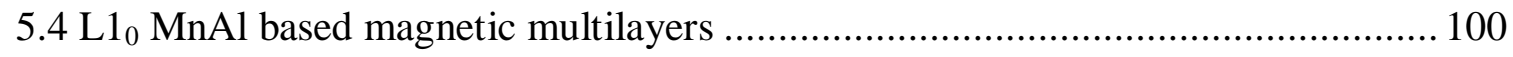

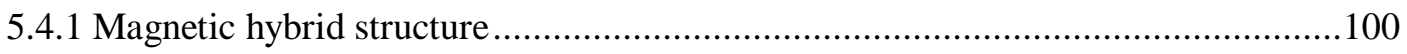

5.4.2 Magneto transport in the hybrid structure .................................................... 101

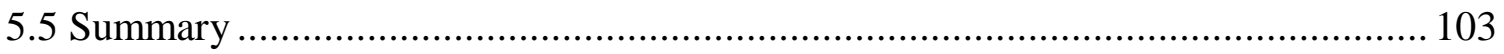

Chapter 6 Current perpendicular to plane (CPP) device fabrication ........ 104

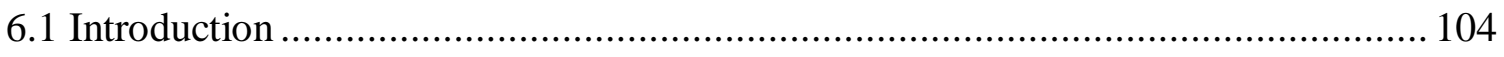

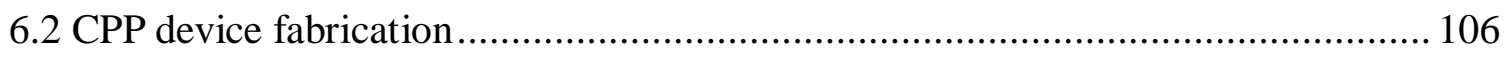

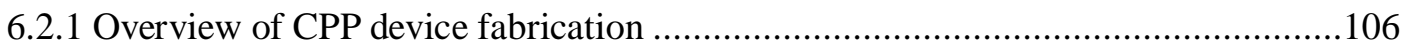

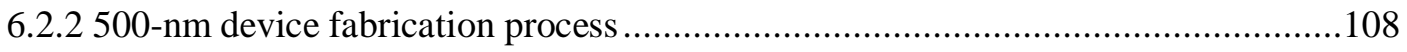

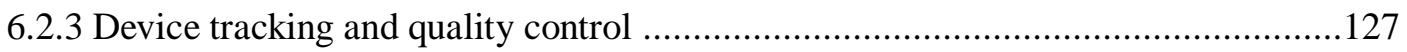

6.2.4 Another example: CPP patterning of magnetic tunnel junctions ..........................132

6.3 Limitations of the CPP fabrication by photolithography ................................ 135

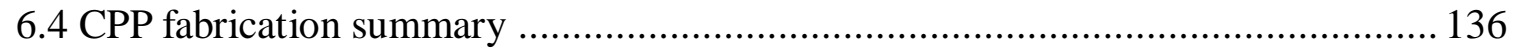

Chapter 7 Spin transfer in $\mathrm{Ni}_{80} \mathrm{Fe}_{20}$ based magnetic multilayers...............137

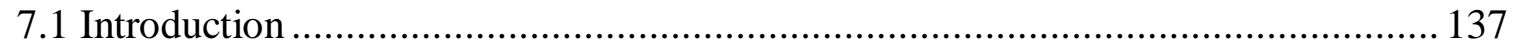

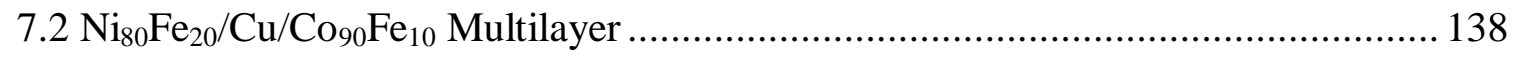


7.2.1 Coercivity tuning for $\mathrm{Co}_{90} \mathrm{Fe}_{10}$ reference layer

7.2.2 Effect of the $\mathrm{Cu}$ spacer thickness

7.2.3 Magneto-transport behaviors of the PSV with out-of-plane magnetic fields

7.3 Spin transfer in nanopillar device

7.3.1 Nanostructure of the STT device.

7.3.2 Coercive field of the nanopillar.

7.3.3 AMR effect of the nanopillar

7.3.4 Current induced magnetization reversal in 500nm pillar.

7.3.5 Comparison with the Slonczewski model

7.3.6 DC current induced magnetization oscillation in $500 \mathrm{~nm}$ pillar

7.4 Micromagnetic simulation

7.4.1 Micromagnetic simulation of switching in a $500 \mathrm{~nm}$ pillar

7.4.2 Micromagnetic simulation of oscillations in a $500 \mathrm{~nm}$ pillar........

7.5 Summary

Chapter 8 Conclusion and future plans

8.1 Research Summary

8.2 Future plans 


\begin{abstract}
The Spin-Torque Nano-Oscillators (STNOs) have been attracting tremendous attention as an innovative nano-scale microwave signal generator for modern electronics systems. The key features include large range of frequency tunability, nanoscale size and compatibility with standard silicon technology. This work explored different magnetic multilayers both from the material perspective and the device aspect with an ultimate goal to lower the critical current density of spin torque switching and to create coherent spin torque oscillation with output power towards the $\mu \mathrm{W}$ range.
\end{abstract}

The major task of this dissertation was to establish the benchmark for spin-torque nano-oscillators fabricated using the Bias Target Ion Beam Deposition (BTIBD) technique and the photolithography techniques. $\mathrm{A} \mathrm{Co}_{2} \mathrm{Fe} \mathrm{Al} / \mathrm{Cr} / \mathrm{Co}_{2} \mathrm{Fe} \mathrm{Al}$ trilayer was evaluated to have a small MR ratio $\sim 0.07 \%$ at room temperature. The small MR ratio will severely affect the output voltage and impede its application on spin-transfer oscillation devices. Optimizations were made on $\mathrm{CFA} / \mathrm{Cu} / \mathrm{CFA}$ and nano-oxide layer(NOL)/CFA/Cu/CFA multilayers to improve the MR ratio. Significant enhancement of MR ratio was observed in the NOL/CFA/Cu/CFA pseudo spin valve (PSV) due to the specular reflection from the NOL. The origin of the magnetoresistance in single layer $\mathrm{L}_{0} \mathrm{MnAl}$ was studied by linking the resistivity change to the domain wall (DW) scattering of charge carriers. Quantitative analysis on remanent states' MFM images and the corresponding resistivity confirmed the 
contribution of DWs to the electric resistivity of MnAl. Magnetic static and magneto transport properties for a magnetic hybrid multilayer configuration $\mathrm{CFA} / \mathrm{Cr} / \mathrm{MnAl}$ have been investigated for the purpose of spin transfer study. Low room temperature MR ratio of $\sim 0.05 \%$ was observed on the hybrid PSV possibly due to the small spin-polarization in the thin MnAl films.

From the device perspective, DC current induced magnetization reversal and magnetization oscillation was observed in $500 \mathrm{~nm}$ large size $\mathrm{Co}_{90} \mathrm{Fe}_{10} / \mathrm{Cu} / \mathrm{Ni}_{80} \mathrm{Fe}_{20}$ pillars. A perpendicular external field enhanced the coercive field separation between the reference layer $\left(\mathrm{Co}_{90} \mathrm{Fe}_{10}\right)$ and free layer $\left(\mathrm{Ni}_{80} \mathrm{Fe}_{20}\right)$ in the pseudo spin valve, allowing a large window of external magnetic field for exploring the free-layer reversal. A magnetic hybrid structure was achieved for the study of spin torque oscillation by applying a perpendicular field $>3 \mathrm{kOe}$. The magnetization precession was manifested in terms of the multiple peaks on the differential resistance curves. Depending on the bias current and applied field, the regions of magnetic switching and magnetization precession on a dynamical stability diagram has been discussed in details. Micromagnetic simulations are shown to be in good agreement with experimental results and provide insight for synchronization of inhomogeneities in large sized device. The ability to manipulate spin-dynamics on large size devices could be proved useful for increasing the output power of the spin-transfer nano-oscillators (STNOs). 


\section{ACKNOWLEDGEMENTS}

The completion of my dissertation would not be possible without the help and support of many people whom I had the privilege of working with. First and foremost, I would like to express my deepest appreciation to my advisor Prof. Stuart A. Wolf for the encouragement and unconditional support he has given to me. I really appreciate his efforts to help me succeed in every aspect of my Ph.D. career. I would like to sincerely thank my co-advisor Prof. Jiwei Lu for his professional mentorship. I thank him for his hands-on guidance on writing of manuscripts and my dissertation. I truly appreciate his unique perspective of raising questions that inspired me to think more deeply about physics. I am also very grateful to Dr. Nam Dao who had given me many suggestions on the design of experiments and results discussion. I would like to thank Prof. Mircea Stan, particularly his student Dr. Mehdi Kabir for all the productive discussion in our collaboration on the STNO project and for sharing with me his knowledge on micromagnetic simulation. I also thank our previous group member Dr. Yishen Cui, who was especially helpful at the beginning of my graduate research. I would also thank Dr. Michael Cyberey for sharing with me his knowledge on nano-size device fabrication techniques in clean room. I would also acknowledge all of the members of NanoStar, Physics Department, UVa clean room and IPM, especially Tonya Reynolds, Dawn Shifflett, Tammie Shifflett, Peter Cline, Vicky

Ingram, Alex Lobo, Joseph Beatrice, Beth Guyton, and Helen McLaughlin for all the 
supporting work that they have done these years to my research. I would like to express my appreciation to Prof. Nilanga Liyanage and the physics department for awarding me a fellowship for my dissertation year. I would also like to thank Prof. Joe Poon and Prof. Israel Klich for being in my review meeting committee for the past five years. I would like to express my special appreciation to all the former and current WOLF/LU group members including Salinporn Kittiwatanakul, Yuhan Wang, Mandy Gu, Nattawut Anuniwat, Wei Chen, Hongxue Liu, Yonghang Pei, Wenjing Yin and Ryan Comes. They are not only excellent teammates that I can seek suggestions for my research but also good friends whom I can ask for personal advice. I would like to thank my wife Mengfan for her support and faith she had in me for my pursuit of Ph.D. degree. Finally, I would like to express my deepest gratitude to my parents who have provided me unconditional love and given me courage and strength along the way in my life. 


\section{LIST OF TABLES}

Table 2-1 Directions of Easy, Medium, and Hard axis in a Cubic Crystal[65] ................38 Table 4-1 The comparison of the CFA coercive fields at different temperatures. Results for two interfaces with \& without NOL are compared........................................ 80

Table 6-1 Outline of the steps used to make CPP nano-pillars at UVa ........................ 128

\section{LIST OF FIGURES}

Figure 1.1 A chart showing the critical current density $\left(J_{C}\right)$ for MTJ switching in comparison with the maximum current that can be provided by a CMOS transistor. The gray line indicates the maximum currents that a CMOS transistor can provide at different sizes. The lines defined by the various symbols are the switching currents as a function of MTJ size for various values of the current densities. A reasonable operation margin (a factor of 4 (Red circle)) can be obtained at a current density of $\sim 5 \times 10^{5} \mathrm{~A} / \mathrm{cm}^{2}$ for a $45 \mathrm{~nm}$ MTJ, which means sufficient current can be provided by a CMOS transistor of the same size at that window. For a smaller junction the margin is even better. (Adapted from NSF EAGER Proposal, Prof. Stuart Wolf, UVa)

Figure 2.1 (a) a schematic plot is shown for the energy band structure of a $d$ transition metal $\mathrm{Cu}$. The density of states $N(E)$ is shown separately for the spin up and down electrons and where a simplified separation has been made between the $4 \mathrm{~s}$ and $3 \mathrm{~d}$ band energies. For the non-magnetic state these are identical for the two spins. All energy levels below the Fermi energy are occupied states (orange and blue). The colored area (orange + blue) corresponds to the total number of conduction electrons in the metal. (b) the corresponding picture is illustrated for Co, with a majority spin chosen to be in the up direction (blue area >orange area). This polarization is indicated by the thick blue arrow 
at the bottom figure to the right. (Adapted from Ref. [21]).....................................

Figure 2.2 Resistance change due to an external magnetic field for Fe/Cr multilayers[19]9 Figure 2.3 Mott's two-current model. (a) A schematic for the parallel (P) magnetization alignment. (b) Illustration of the electronic band structure for parallel alignment (the majority spin directions are indicated by two thick blue arrows at the bottom) (c) Equivalent circuit for the resistance of parallel alignment of magnetization. (d) A schematic for the antiparallel (AP) magnetization alignment. (e) Illustration of the electronic band structure for antiparallel alignment (the majority spin directions are indicated by the thick blue and orange arrows) (f) Equivalent circuit for the resistance of parallel alignment of magnetization. [Adapted based on Prof. Stu Wolf's lecture notes (UVa, 2013 Spring) and Ref.[21]] .11

Figure 2.4 Schematic diagram showing the formation of exchange bias. (i)-(v) shows the spin configuration of an FM/AFM bilayer at different stage of an exchange biased hysteresis loop. Note that the spin configurations are just a simple cartoon to illustrate the effect of the coupling and they are not necessarily accurate portraits of the actual rotation of the FM or AFM magnetizations. (Adapted from Ref. [29]). .14

Figure 2.5 Schematic of the spin-dependent tunneling process for (a) parallel and (b) anti-parallel states, according to Jullière's model. Dashed lines indicate the spin-conserved tunneling. (Reproduced from Ref.[29]).

Figure 2.6 Schematic showing electrons being spin-polarized along $S 1$ by the bottom magnetic layer and, in the act of rotating toward the new polarization direction $S 2$ in the free layer, transferring spin momentum to the free layer magnetic moment. If this torque is large enough it can overcome the damping and switch the magnetization of the free layer, or it can balance out the damping and leads to stable precession........................ 18

Figure 2.7 Simple illustration of the five-layer spin transfer system proposed by Slonczewski.(Adapted from Ref.[33]) 20 Figure 2.8 Typical DC current induced magnetization switching in magnetic multilayer nanopillars. The size of the pillar is shown in the SEM images. (Adapted from Prof. Stu

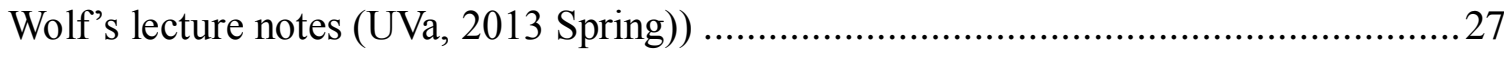

Figure 2.9 (a) Schematic diagram of MRAM using MTJ. (b) Schematic diagram of 
STT-MRAM. [Adapted from Prof. Stu Wolf's lecture notes (UVa, 2013 Spring)] .30

Figure 2.10 (a) Differential resistance versus current at different magnetic fields of 0(bottom), $0.5,1.0,1.5,2.0$ and $2.5 \mathrm{kOe}$. (b) RF power density spectra normalized by square of the injected current. The emission was observed for a GMR nano-pillar with in-plane magnetization under $-2 \mathrm{kOe}$ of the external field. (Adapted from Ref. [4]) .......31 Figure 2.11 Dynamic stability diagram obtained from the $\mathrm{Co} / \mathrm{Cu} / \mathrm{Co}$ in-plane nano-pillars. (Adapted from Ref. [4]). .33

Figure 2.12 (a) Schematic cross section of the magnetic point contact. (b) Cross-sectional SEM image of the two 80-nm-diameter contacts that were used to observe mutual coupling of the STO. (c) SEM top image of the coupled point contacts. (Adapted from Ref.[57])

Figure 2.13 (a) Magnetization curves for single crystals of nickel with cubic structure. (b) Magnetization curves for singe crystal of cobalt with hexagonal close-packed structure. (Adapted from Ref. [65])

Figure $2.14 \mathrm{L1}_{0}$ structure of $\tau-\mathrm{MnAl}$, which can be reduced into a distorted $\mathrm{B} 2$ structure. (Adapted from Ref.[95]). 45

Figure 3.1 Schematic layout of Mark II End-Hall ion source and HCES 5000 Hollow Cathode electron source. (Reproduced from Ref.[95]) 49

Figure 3.2 The schematic of the RBTIBD processing chamber. (Reproduce from Ref. [95]) 49

Figure 3.3 Schematics of the pulsed DC bias control. (Reproduced from Ref.[29]).......51 Figure 3.4 (a) SEM image for a new AFM tip; the inset shows the very end of the tip. (b) SEM image of a worn-out tip. The inset shows the end of tip with a flattened triangle shape.

Figure 4.1 (a) Hysteresis loops of a single layer CFA layer with $t=10 \mathrm{~nm}$. (b-f) Hysteresis loops for samples with different $\mathrm{Cr}$ thickness varied from $1.5 \mathrm{~nm}$ to $6 \mathrm{~nm}$. The samples were measured at room temperature.

Figure 4.2 MR and the corresponding hysteresis loop of the trilayers with $\mathrm{Cr}$ spacer thickness of $1.5 \mathrm{~nm}$. The curves are measured at different temperature varying from $300 \mathrm{~K}$ to $50 \mathrm{~K}$. 
Figure 4.3 (a) Experimental (black line with squares) and calculated hysteresis loops (red line) of the trilayer with $\mathrm{Cr}$ thickness $1.5 \mathrm{~nm}$ measured at different temperatures from $300 \mathrm{~K}$ to $50 \mathrm{~K}$. $J_{1}$ is assumed as a constant of $-0.006 \mathrm{erg} / \mathrm{cm}^{3}$. (b) Calculated $-J_{2}$ as a function of the measurement temperature. The blue triangles indicate the value for $-J_{1} . .68$ Figure 4.4 For the sample with a $\mathrm{Cr}$ thickness of $1.5 \mathrm{~nm}$, the experimental determined remanence $M_{r} / M_{s}$ ratio and $H s$ plotted as a function of temperature from $300 \mathrm{~K}$ to $50 \mathrm{~K}$.

Figure 4.5 MR for the trilayer sample measured at room temperature $(300 \mathrm{~K})$ and $50 \mathrm{~K}$. The $\mathrm{Cr}$ spacer thickness is $1.5 \mathrm{~nm}$ for the sample. The black and green arrows indicate the field sweeping direction. The schematic diagrams illustrate the corresponding magnetic states (states \#1 to \#4) for different magnetoresistance regime on the MR curve guided by green arrows.

Figure 4.6 The hysteresis loops for sample A and B, corresponding to the structure $\mathrm{MgO}$ (substrate)// CFA(11 nm) and $\mathrm{MgO}($ substrate $) / / \mathrm{Ru}(20 \mathrm{~nm}) / \mathrm{CFA}(11 \mathrm{~nm})$ respectively.

Figure 4.7 AFM image showing the topography of sample A and B. The roughness is given by the RMS value.

Figure 4.8 The hysteresis loops for samples with different $\mathrm{Cu}$ spacer thicknesses were measured at room temperature. The structure for the sample was $\mathrm{MgO}$ (substrate)// $\mathrm{Ru}(20 \mathrm{~nm}) / \mathrm{CFA}(3.5 \mathrm{~nm}) / \mathrm{Cu}(\mathrm{x}) / \mathrm{CFA}(3.5 \mathrm{~nm}) / \mathrm{Ru}(3 \mathrm{~nm})$. .74

Figure 4.9 MR for the CFA/Cu5/CFA sample before \& after annealing measured at 300K.

Figure 4.10 The hysteresis loop (a) and the corresponding MR curve (b) measured at room temperature. The sample was grew on $\mathrm{SiO}_{2}$ substrate with a structure $\mathrm{Ru} 20(\mathrm{~nm}) /$ $\mathrm{Co}_{2} \mathrm{FeAl}(3.5 \mathrm{~nm}) / \mathrm{Cu}(6 \mathrm{~nm}) / \mathrm{Co}_{2} \mathrm{FeAl}(1.7) / \mathrm{Ru}(3)$ 77

Figure 4.11 $M-H$ loop for the NOL/CFA/Cu/CFA sample measured at $150 \mathrm{~K}$ 80 Figure 4.12 MR for the NOL/CFA/Cu/CFA sample measured at $150 \mathrm{~K}$. The arrows indicate the field sweeping direction. The sample was annealed at $350{ }^{\circ} \mathrm{C}$ for 12 seconds under high vacuum.

Figure 4.13 Schematic drawing of the spin diffusion length. During the propagation of xiii 
the electron, the electron moves opposite to the electric field $E$ on average. The average distance between two scattering events is the mean free path. For metals at room temperature, the typical mean free path is about $10 \mathrm{~nm}$. The average distance between two spin-flip events corresponds to the spin diffusion length $l_{\mathrm{sf}}$. (Adpted from Ref. [133])

Figure 5.1 2ө XRD scan of MnAl deposited on Cr-buffered $\mathrm{MgO}$ substrate showing the fundamental (002) and superlattice (001) peaks. 87

Figure 5.2 $\Phi$ scans on $\mathrm{L}_{0} \mathrm{MnAl}$ deposited on a Cr-buffered $\mathrm{MgO}$ substrate at a growth temperature $200{ }^{\circ} \mathrm{C}$. The green line represents data taken at $2 \theta$ of $41.12^{\circ}(\mathrm{MnAl}(011)$ peak), the red one for data taken at $44.17^{\circ}(\mathrm{Cr}$ (011) peak) and the blue one for data taken at $62.27^{\circ}$ ( $\mathrm{MgO}(022)$ peak). The right side is the schematic diagram of the epitaxial relationships at $\mathrm{MgO} / \mathrm{Cr}$ and $\mathrm{Cr} / \mathrm{MnAl}$ interfaces. 88

Figure 5.3 In-plane (Blue triangles) and out-of-plane (Red dots) hysteresis loops for $\mathrm{MnAl}$ films measured at room temperature.

Figure $5.42 \mu \mathrm{m} \times 2 \mu \mathrm{m}$ (a) AFM topography image for the MnAl films and (b) the corresponding MFM image (or phase image) after demagnetized at $30 \mathrm{kOe}$ along the perpendicular direction. The surface roughness RMS is around $2 \AA . . . . \ldots \ldots \ldots \ldots \ldots \ldots \ldots . . . . . . . . . .91$ Figure 5.5 MR curves on a $50 \mu \mathrm{m}$ wide Hall bar measured at temperature from $50 \mathrm{~K}$ to $320 \mathrm{~K}$. The external field was applied out of plane. The inset shows the Hall bar pattern with the longitudinal measurement configuration .93 Figure 5.6 (a) Coercivity (Red dots) versus the MR peak positions (Blue squares) for temperature range $175 \mathrm{~K}$ to $320 \mathrm{~K}$. The inset shows the Hall resistivity versus the perpendicular applied field at $300 \mathrm{~K}$. (b) The MR enhancement at the coercive field and $\left(\rho_{x y} / \rho_{x x}\right)^{2}$ vs. temperature.

Figure 5.7 MR curves measured at temperature $250 \mathrm{~K}$ (Blue dots) and $50 \mathrm{~K}$ (Red triangles). The external field was applied in plane and along the longitudinal direction..96 Figure 5.8 (a) The resistivity measured with the field applied in the longitudinal direction (Red dots) and the perpendicular direction (Blue triangles), corresponding to $\rho_{\text {long }}$ and $\rho_{\text {perp }}$ respectively. The remanent states are marked as A and B. (b)\&(c) MFM phase images corresponding to remanent states $\mathrm{A}$ and $\mathrm{B}$. The domain boundaries have been 
highlighted in green color. Density of domains in state A: $5.57 / \mu \mathrm{m}^{2}$ and B: $4.67 / \mu \mathrm{m}^{2} \ldots .98$ Figure 5.9 In-plane (a) and Out-of-plane (b) hysteresis loops of the magnetic hybrid structure Cr40nm / MnAl20nm / Cr2nm / $\mathrm{Co}_{2} \mathrm{FeAl} 3.5 \mathrm{~nm} / \mathrm{Ru} 5 \mathrm{~nm}$ measured at different temperatures from $300 \mathrm{~K}$ to $50 \mathrm{~K}$. 101

Figure 5.10 (a) M-H loop of the pseudo spin-valve Cr40nm / MnAl20nm / Cr2nm / $\mathrm{Co}_{2} \mathrm{FeAl3} .5 \mathrm{~nm} / \mathrm{Ru} 5 \mathrm{~nm}$ at $150 \mathrm{~K}$. (b) The corresponding MR(in $\Omega$ ) measured at $150 \mathrm{~K}$.

Figure 6.1 (a) Schematic of a CIP patterning structure. (b) A Hall bar structure with CIP geometry. (c) Schematic of a CPP patterning structure. (d) A magnetic structure with CPP

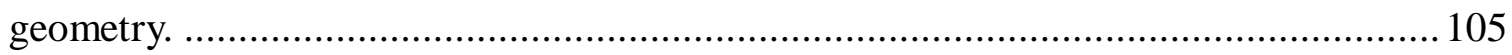

Figure 6.2 The multilayer structure deposited by BTIBD, the Au/Ti layer is deposited afterward. 110

Figure 6.3 Photolithography masks. (a) Layer 1 is to define the bottom electrodes. (b) STNO pillars are defined by layer 2. (c) Layer 3 is to open the contact area in the passivation layer. (d) Layer 4, to define the top contact pads. 110 Figure 6.4 (a) A schematic showing the cross section of the bottom electrode. (b) A SEM image of the bottom electrode.

Figure 6.5 (a) A $5 \mu \mathrm{m} \times 5 \mu \mathrm{m}$ AFM scan on the edge of an etched step. (b) A 3D view of the step with scale showing the etching depth of $12.53 \mathrm{~nm}$. 116 Figure 6.6 To precisely monitor the ion-milling, the hysteresis loop of the test sample has been measured before and after etching. Quantitative information such as coercive fields, magnetization $\left(M_{\mathrm{NiFe}}, M_{\mathrm{CoFe}}\right)$ can be extracted from the Hloop, which are used to determine the time for the etching endpoint. (a) The hysteresis loop measured before ion milling. (b) The hysteresis loop measured after the layers above CoFe have been removed.

Figure 6.7 (a) A SEM image showing the photoresist pillar after development. The inset shows the sidewall re-deposition after ion milling. (b) A resist pillar with over-exposure. (c) A V-neck shape resist pillar with under-exposure time. (d) Comparison of the same resist pillar before and after etching. 122

Figure 6.8 Schematic diagram showing the cross section of the device. The photoresist 
(PR) remains above the pillar after etching.

Figure 6.9 Deposition of $\mathrm{SiO}_{2}$ to cover the bottom electrodes and to insulate the sidewall of the device pillar.

Figure 6.10 Side view and top view showing a crater-shape opening for the device pillar that is buried and surrounded by the $\mathrm{SiO}_{2}$ (Schematically demonstrated in Figure 6.9). The brighter circle seen in the top-view is due to the charge accumulation during the SEM scan. 125

Figure 6.11 Schematic showing the device after the $\mathrm{SiO}_{2}$ over the bonding pads has been removed with a buffered oxide etch (BOE) etch.

Figure 6.12 (a) A microscope image showing an array of devices after the seed layer have been removed. (b) The STNO device with special designed contact pads. 128

Figure 6.13 Resistance versus temperature curve is used to evaluate the fabrication quality. (a) A linear decreasing curve indicates a metallic structure. (b) The abnormal R-T curve could indicate the remaining of PR above the pillar. The sharp jump on $\mathrm{R}$ is coming from the loose contact of wire bonding.

Figure 6.14 MTJ photolithography mask. (a) Layer 1 is to define the bottom contacts. (b) MTJ pillars with various aspect ratios are defined by layer 2. (c) Layer 3 is to open the via area in the passivation layer. (d) Layer 4, to define the top contact.

Figure 6.15 Flow chart of MTJ fabrication process: (a) Definition of the bottom contact. (b) Definition of the junction through ion milling down to the bottom Ru contact layer. (c) Definition of the via and top contact pads by $\mathrm{SiO}_{2}$ passivation and Au evaporation...... 134 Figure 6.16 Optical image showing elongated MTJ pillars with different aspect ratio of $10 \times 50 \mu \mathrm{m}^{2}($ Left $)$ and $10 \times 20 \mu \mathrm{m}^{2}$ (Right) 135

Figure 7.1 (a) The hysteresis loops showing thickness dependent $H_{c}$ for $\mathrm{Co}_{90} \mathrm{Fe}_{10}$ single layers. (b) The plot of coercivie fields for $\mathrm{Co}_{90} \mathrm{Fe}_{10}$ versus the thicknesses. 139 Figure 7.2 Experimental hysteresis loops of samples (a-d) with different $\mathrm{Cu}$ thickness varied from $3 \mathrm{~nm}$ to $8 \mathrm{~nm}$. The samples were measured at room temperature with the magnetic field applied in plane. The arrow marks the transition for $\mathrm{Ni}_{80} \mathrm{Fe}_{20}$ and $\mathrm{Co}_{90} \mathrm{Fe}_{10}$.

Figure 7.3 (a) Magnetoresistance measured by Van der Pauw configuration on the full 
film with $\mathrm{Cu}=8 \mathrm{~nm}$. (b) The MR ratios for the PSV (as shown inset) with different $\mathrm{Cu}$ thickness are plotted together for comparison.

Figure 7.4 RT magnetoresistance curve (solid red) measured on the $20 \mathrm{~nm} \mathrm{Ru} / 2.2 \mathrm{~nm}$ $\mathrm{Co}_{90} \mathrm{Fe}_{10} / \mathbf{5 n m} \boldsymbol{C u} / 6.5 \mathrm{~nm} \mathrm{Ni}{ }_{80} \mathrm{Fe}_{20} / 5 \mathrm{~nm} \mathrm{Ru}$ PSV structure with an out-of-plane magnetic field. The blue curve shows the corresponding hysteresis loop on the out-of-plane direction.

Figure 7.5 (a) Zoom in on the low-field peaks in Figure 7.4. (b) Magnetoresistance curve on the same PSV measured with in-plane field. The arrows indicate the direction of the field sweeping. The dashed circle indicates the scale for the coercive field. (c) Rotation angle curves for structure $20 \mathrm{~nm} \mathrm{Ru} / 6.5 \mathrm{~nm} \mathrm{Ni}{ }_{80} \mathrm{Fe}_{20} / 5 \mathrm{~nm} \mathrm{Ru}$ (blue triangles) and structure 20nm Ru/2.2nm $\mathrm{Co}_{90} \mathrm{Fe}_{10} / 5 \mathrm{~nm} \mathrm{Ru}$ (red dots). The schematic diagrams show the relative magnetic configuration for the two layers. The black arrows indicate the position for the maximum angle difference between the two layers' magnetization. 145 Figure 7.6 (a) Schematic representation of the patterned pseudo-spin-valve sample. The bottom $\mathrm{Co}_{90} \mathrm{Fe}_{10}$ layer is partially patterned. The arrow shows the electron flow direction for negative current. (b) A SEM image of the patterned pillar with a diameter of $500 \mathrm{~nm}$.

(c) The optical microscope image of a device with CPW contacts.

Figure 7.7 Current perpendicular to plane (CPP) MR curve on the patterned sample. The black arrows show the direction of the field sweep.

Figure 7.8 Sheet resistance $\mathrm{R}_{\mathrm{S}}$ versus applied magnetic field $\mathrm{H}$ of the continuous $\mathrm{Co}_{90} \mathrm{Fe}_{10}$ layer, measured with the magnetic field perpendicular to the film plane, at room temperature.

Figure 7.9 Differential resistance versus biased direct current $\left(\mathrm{I}_{\mathrm{B}}\right)$ with out-of-plane magnetic fields of 0 (bottom, red) and 200 Oe (blue). The current sweep starts at 0mA and the arrows indicate the direction of the current sweep. The sharp transition happens at $I_{\mathrm{C}}{ }^{\mathrm{P}-\mathrm{AP}}=-48 \mathrm{~mA}$ (current density of $2.45 \times 10^{7} \mathrm{~A} / \mathrm{cm}^{2}$ ) and $I_{\mathrm{C}}{ }^{\mathrm{AP}-\mathrm{P}}=47 \mathrm{~mA}$ (current density of $2.39 \times 10^{7} \mathrm{~A} / \mathrm{cm}^{2}$ ) for $H=0$. At $H=200 \mathrm{Oe}$, the switching currents are $I_{\mathrm{C}}{ }^{\mathrm{P}-\mathrm{AP}}=$ $-47.2 \mathrm{~mA}$ (current density of $2.40 \times 10^{7} \mathrm{~A} / \mathrm{cm}^{2}$ ) and $I_{\mathrm{C}}{ }^{\mathrm{AP}-\mathrm{P}}=46 \mathrm{~mA}$ (current density of $2.35 \times 10^{7} \mathrm{~A} / \mathrm{cm}^{2}$ ). The inset shows the minor MR hysteresis loop for $\mathrm{Ni}_{80} \mathrm{Fe}_{20}$ layer (free layer). The black arrows indicate the direction of the field sweep. 152 
Figure 7.10 Critical current for reversal of magnetization between the AP and P states at different fields, where the circles (red) correspond to the critical current for AP-P transition $J_{C}{ }^{A P-P}$ and squares (blue) correspond to the critical current for P-AP transition $J_{C}^{P-A P}$.

Figure $7.11 d V / d I$ versus $I_{B}$ for (a) $H=3.5 \mathrm{kOe}$ and (b) $H=4.0 \mathrm{kOe}$, the scans are reversible with both current directions. The inset shows the second derivative of $d V / d I$ indicating the relative amplitude, width and position of the peaks. 156 Figure 7.12 Experimentally determined $J_{B}-H$ (Out-of-Plane field) dynamic stability diagram for the patterned sample showing the parallel or antiparallel states where each symbol corresponds to a distinct change in the resistance. Below $3.2 \mathrm{kOe}$, the symbols are from hysteretic switching where the circles (red) are $J_{C}{ }^{A P-P}$ and squares (blue) are $J_{C}$ $P-A P$. For field larger than $3.2 \mathrm{kOe}$, the triangles (green) refer to the peaks on the differential resistance curves. The dashed line corresponds to the reversible peaks at $H=$ $3.5 \mathrm{kOe}$ [Figure 7.11(a)].

Figure 7.13 Micromagnetic simulation showing the evolution of $\mathrm{m}_{\mathrm{x}}$ component at a current density of $3 \times 10^{7} \mathrm{~A} / \mathrm{cm}^{2}$ with an out-of-plane field $\mathrm{H}=200$ Oe. The flip-over of a $C$-state is demonstrated by the spatial magnetization distribution $1 \rightarrow 2 \rightarrow 3 \rightarrow 4$. The cores are added for illustration purpose.

Figure 7.14 (a) The temporal magnetization component evolutions of $m_{x}$ with different current densities: $3.0 \times 10^{7} \mathrm{~A} / \mathrm{cm}^{2}$ (red), $2.0 \times 10^{7} \mathrm{~A} / \mathrm{cm}^{2}$ (green) and $1.0 \times 10^{7} \mathrm{~A} / \mathrm{cm}^{2}$ (blue). (b) The simulated evolution of the total energy for injected current density of $3.0 \times 10^{7} \mathrm{~A} / \mathrm{cm}^{2}$ (red) and $1.0 \times 10^{7} \mathrm{~A} / \mathrm{cm}^{2}$ (blue). The inset shows a schematic diagram of the energy barrier between the parallel and antiparallel state. 162

Figure 7.15 The magneto-static simulation results showing the spatial magnetization distribution with cross-section view for the whole structure (a) and top view for the free layer (b)

Figure 7.16 (a) The temporal magnetization component evolutions of $m_{x}, m_{y}$ under an out-of-plane field of $3.5 \mathrm{kOe}$ and with a constant current density of $3.0 \times 10^{7} \mathrm{~A} / \mathrm{cm}^{2}$. (b) The precession orbits of the free layer magnetization. (c)\&(d) The evolution of the spatial magnetization distribution at the corresponding time marked under them. The 
non-coherent process as shown in (c) features a non-uniform multiple domain oscillation while (d) features a coherent magnetization rotation. 163 Figure 7.17 At $H=3.5 \mathrm{kOe}$, different current density leads to distinct stabilized precession orbits and frequencies. The main precession frequency $f$ is determined by taking the Fourier transforms of $m_{x}$. At $2.0 \times 10^{7} \mathrm{~A} / \mathrm{cm}^{2}$ (black), $3.0 \times 10^{7} \mathrm{~A} / \mathrm{cm}^{2}$ (red) and $3.4 \times 10^{7} \mathrm{~A} / \mathrm{cm}^{2}$ (blue), the simulated oscillation has the frequency of $3.15 \mathrm{GHz}, 10.5 \mathrm{GHz}$ and $14.6 \mathrm{GHz}$ respectively. 165

Figure 7.18 (a) The temporal magnetization component evolutions of $m_{x}, m_{y}$ under an out-of-plane field of $5.0 \mathrm{kOe}$ and with a constant current density of $3.0 \times 10^{7} \mathrm{~A} / \mathrm{cm}^{2}$. (b) The precession orbits of the free layer magnetization. (c) The evolution of the spatial magnetization distribution at the corresponding time marked under them. 167

Figure 8.1 Schematic diagram of the all-perpendicular structure and the corresponding In-plane (blue) and out-of-plane (red) $M-H$ loops for the all-perpendicular pseudo spin-valve. The $M-H$ curves are measured at room temperature. 


\section{Chapter 1 Introduction}

\subsection{An overview of the dissertation}

\subsubsection{Motivation}

The last few decades have witnessed a continuous race for miniaturization and cost reduction of electronic components, usually described by "Moore's law". Lately, spintronics has emerged as an extremely fruitful direction of research beyond Moore's law, and application of the spin degree of freedom of the electron due to its compatibility with the exponentially expanding CMOS electronics [1]. One of the latest major applications of spintronics is the development of Spin Transfer Torque Magneto-resistive Random Access Memory (STT-MRAM) that can surpass mainstream DRAM memories because of its compatibility with conventional CMOS processing and its non-volatile properties [2].

One of the key issues of STT-MRAM technology is the reduction in the STT writing current density of the magnetic tunnel junction storage cell. As shown in Figure 1.1, the integration of MRAM cells with CMOS transistors requires a writing current density lower

than $5 \times 10^{5} \mathrm{~A} / \mathrm{cm}^{2}$. However, to date, in the best case, the critical current density required with current MTJ materials remains above $1 \times 10^{6} \mathrm{~A} / \mathrm{cm}^{2}[3]$--- still too high for application, hence, more innovations are needed in engineering the magnetic multilayer structures and to optimize the ferromagnetic material's intrinsic properties. 


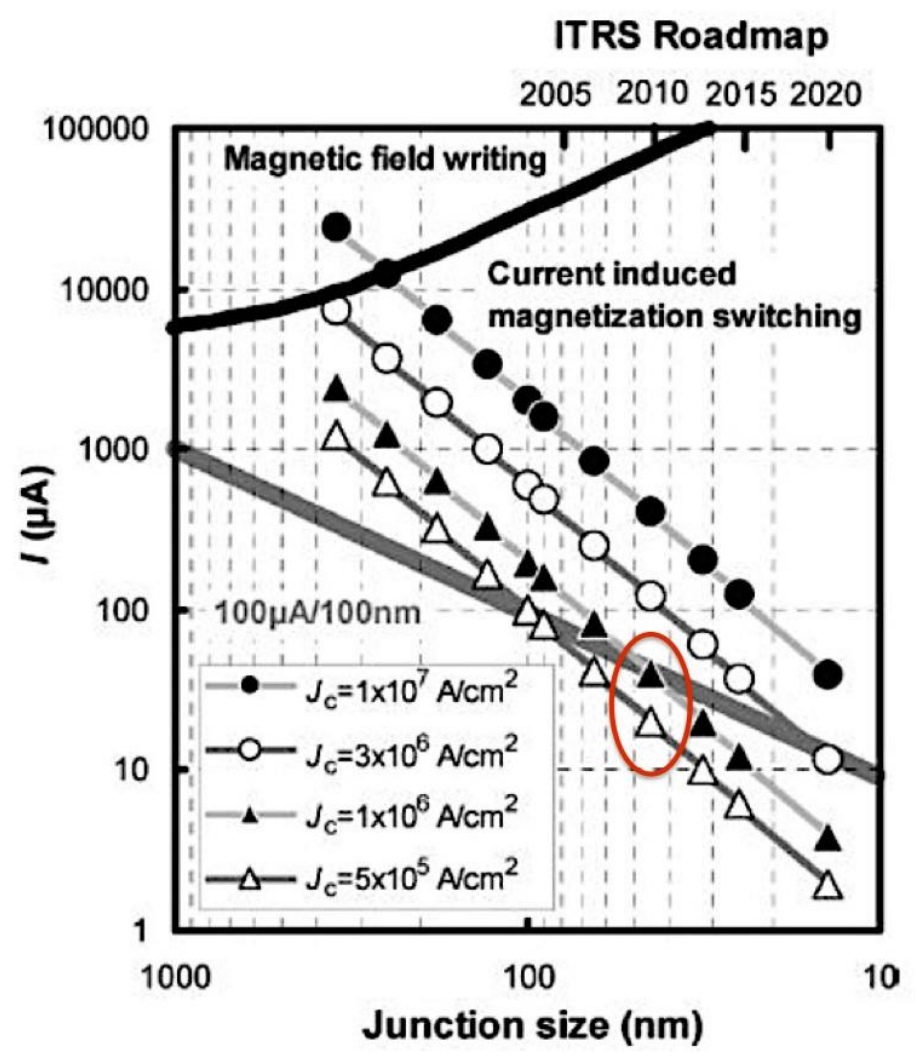

Figure 1.1 A chart showing the critical current density $\left(J_{C}\right)$ for MTJ switching in comparison with the maximum current that can be provided by a CMOS transistor. The gray line indicates the maximum currents that a CMOS transistor can provide at different sizes. The lines defined by the various symbols are the switching currents as a function of MTJ size for various values of the current densities. A reasonable operation margin (a factor of 4 (Red circle)) can be obtained at a current density of $\sim 5 \times 10^{5} \mathrm{~A} / \mathrm{cm}^{2}$ for a 45 nm MTJ, which means sufficient current can be provided by a CMOS transistor of the same size at that window. For a smaller junction the margin is even better. (Adapted from NSF EAGER Proposal, Prof. Stuart Wolf, UVa) 
Another spintronics application, Spin-Torque Nano-Oscillators (STNOs), has been attracting tremendous attention as an innovative nano-scale microwave signal generator for modern electronics systems. The microwave oscillations generated by the STNOs are highly tunable by adjusting the DC current and applied magnetic field, with frequencies ranging from a few $\mathrm{GHz}$ to $100 \mathrm{GHz}$ and linewidths on the order of $\sim 100 \mathrm{MHz}[4,5]$. Furthermore, the nanometer sized devices are among the smallest microwave oscillators yet developed[6] and their compatibility with standard silicon processing opens the possibility for on-chip applications [7, 8]. However, the bottlenecks for the widespread application of STNOs lies in the enhancement of the output power above the current limit of $\sim 0.5 \mu \mathrm{W}[9]$.

For magnetic multilayers, nano-pillars incorporating perpendicular magnetic anisotropy (PMA) ferromagnets, there are predictions for a reduced critical current density and an increased speed of switching [10-12]. Moreover, such devices would be able to generate oscillations requiring low or even zero external magnetic fields [13-17]. Given this context, this dissertation is focused on the exploration of innovated magnetic multilayers systems utilizing PMA materials with an ultimate goal to lower the switching current density of STT devices and to create coherent oscillation with output powers larger than $0.5 \mu \mathrm{W}$. 


\subsubsection{Outline}

This dissertation is dedicated to the study of spin-transport in magnetic multilayers and magnetic nanostructures. Several magnetic multilayer systems have been explored in this work for the purpose of benchmarking the spin-torque nano-oscillators.

In Chapter 2, I will introduce the fundamental physics of spintronics and the discovery of the spin-transfer torque effect. The Slonczewski model will be outlined to quantify the spin transfer effects in magnetic multilayer systems. I will also detail the impact of the spin-transfer effect on STT-MRAM and on the development of STNOs. Fundamental properties and characterization of perpendicular magnetic anisotropy materials will also be discussed.

Experimental techniques used in this work including thin film fabrication and characterizations are described in Chapter 3 to familiarize the reader with the systems.

Chapter 4 presents the study on $\mathrm{Co}_{2} \mathrm{FeAl}$ based magnetic multilayer structures. The dependence of interlayer couplings on temperature in the pseudo spin-valve is studied in $\mathrm{CFA} / \mathrm{Cr} / \mathrm{CFA}$ trilayers. $\mathrm{CFA} / \mathrm{Cu} / \mathrm{CFA}$ tri-layers have also been made to study how a spacer layer of $\mathrm{Cu}$ would affect the interlayer coupling and the MR ratio compared with a $\mathrm{Cr}$ spacer. The epitaxial growth of the CFA layer was suggested to be crucial to obtain a high spin-polarization for CFA and hence a high MR ratio. A CFA/Cu/CFA pseudo spin-valve with a nano-oxide layer (NOL) shows promise to significantly increase the MR ratio and the mechanism is discussed in detail. 
Chapter 5 is focused on $\mathrm{L} 1_{0} \mathrm{MnAl}$ with strong perpendicular magnetic anisotropy. The magneto-transport properties of MnAl as a function of temperature was studied in a Hall bar patterned sample. An MR enhancement was attributed to the increase in the magnetic domain walls based on a quantitative correlation between the domain wall density and the resistivity. Spin-transport on $\mathrm{L}_{0} \mathrm{MnAl}$ based magnetic multilayer, including a $\mathrm{MnAl} / \mathrm{Cr} / \mathrm{CFA}$ magnetic hybrid structure is discussed.

As the spin-transfer effect is primarily studied in devices where the current is run perpendicular to the plane of the magnetic layers, in Chapter 6, I will describe the fabrication techniques developed at UVa to fabricate nano-size and micro-size current-perpendicular-to-plane (CPP) structures. I used photolithographic techniques to fabricate magnetic pillars with electrodes oriented in such a way as to allow current to run perpendicular to the magnetic layers. Using the fabrication technique outlined in that chapter, I successfully fabricated $500 \mathrm{~nm}$ nanopillar devices.

In Chapter 7, the spin-transfer effect is benchmarked in the NiFe based multilayer structure deposited by the Biased Target Ion Beam Deposition (BTIBD) system. I discuss the spin transfer effect including the spin-torque induced magnetization reversal and magnetization oscillation that is studied in $500 \mathrm{~nm}$ large size $\mathrm{Co}_{90} \mathrm{Fe}_{10} / \mathrm{Cu} / \mathrm{Ni}_{80} \mathrm{Fe}_{20}$ pillars. A perpendicular external field enhanced the coercive field separation between the reference layer $\left(\mathrm{Co}_{90} \mathrm{Fe}_{10}\right)$ and free layer $\left(\mathrm{Ni}_{80} \mathrm{Fe}_{20}\right)$ in the pseudo spin valve, allowing a large window of external magnetic field for exploring the free-layer reversal. A magnetic 
hybrid structure was achieved for the study of spin torque oscillation by applying a perpendicular field $>3 \mathrm{kOe}$. The magnetization precession was manifested in terms of the multiple peaks on the differential resistance curves. Depending on the bias current and applied field, the regions of magnetic switching and magnetization precession on a dynamical stability diagram has been discussed in detail. Finally, I carried out micromagnetic simulations based on the Landau-Lifshitz-Gilbert-Slonczewski (LLGS) equation to better interpret the magnetization switching and oscillations observed experimentally on the large sized nanopillar. Micromagnetic simulations are shown to be in good agreement with experimental results and provide insight for synchronization of inhomogeneities in large sized device.

The dissertation is summarized in Chapter 8 along with directions for future work. 


\section{Chapter 2 Background}

\subsection{Giant magnetoresistance (GMR)}

GMR is one of the most important phenomenon that spintronics is based on. It is caused by spin-dependent scattering [18]. The effect typically happens in multilayer structures composed of ferromagnetic materials, such as $\mathrm{Fe}$, $\mathrm{Co}$, Ni or their alloys separated by non-magnetic metal interlayers such as $\mathrm{Cu}$ or $\mathrm{Cr}$ [18-20]. The phenomenon originates from the spin-split energy bands that form a different density of states (DOS) at the Fermi surfaces for spin-up and spin-down electrons. The density of states, $N(E)$, represents the number of electrons in the system having energy within the interval ( $E$, $E+d E)$.

a)

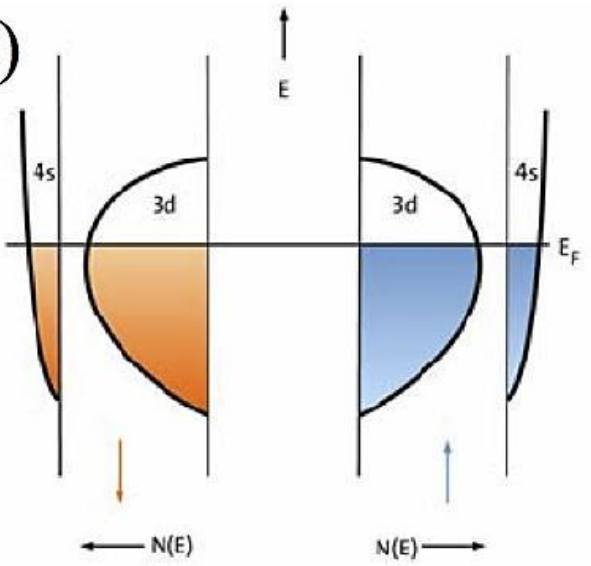

b)

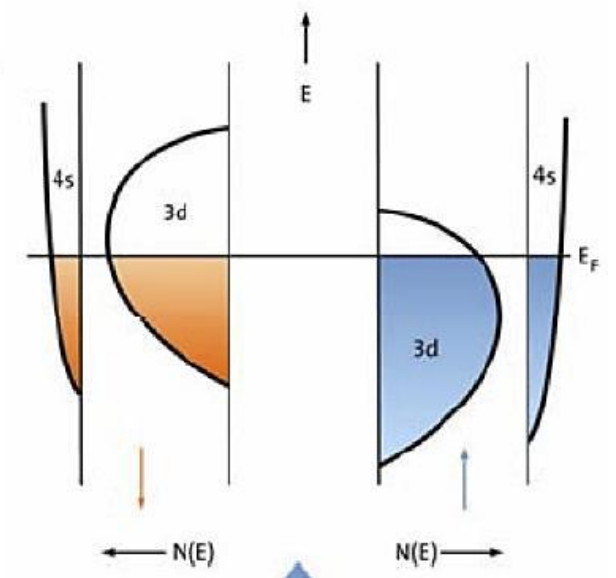

Figure 2.1 (a) a schematic plot is shown for the energy band structure of a $d$ transition metal $\mathrm{Cu}$. The density of states $N(E)$ is shown separately for the spin up and down 
electrons and where a simplified separation has been made between the $4 \mathrm{~s}$ and $3 \mathrm{~d}$ band energies. For the non-magnetic state these are identical for the two spins. All energy levels below the Fermi energy are occupied states (orange and blue). The colored area (orange + blue) corresponds to the total number of conduction electrons in the metal. (b) the corresponding picture is illustrated for Co, with a majority spin chosen to be in the up direction (blue area >orange area). This polarization is indicated by the thick blue arrow at the bottom figure to the right. (Adapted from Ref. [21])

Figure 2.1 shows schematically the density of states (DOS) of $\mathrm{Cu}$ and $\mathrm{Co}$. The electronic states are composed of wide $s$-bands and narrow $d$-bands. For $\mathrm{Cu}$, there are equal numbers of spin up and spin down electrons ending up with no net magnetization, and the spin polarization is zero. Spin polarization is one of the most important parameters in spintronics and is defined as [22]:

$$
\mathrm{P}=\frac{\mathrm{N} \uparrow-\mathrm{N} \downarrow}{\mathrm{N} \uparrow+\mathrm{N} \downarrow}
$$

where $N \uparrow(N \downarrow)=$ density of states at the Fermi level for spin up (down). For ferromagnetic metal this leads to a net spin polarization $0<P \leq 1$.

The conductivity depends on the spin due to the strong spin-dependent scattering that occurs on the N/F interface. Considering electron transport through the non-magnetic metal/ferromagnet/non-magnetic metal structure, the conductivity is mainly determined by the incident un-polarized 4 s electrons which are easily mobile due to the large energy range 
of the bands. As illustrated in Figure 2.1(b), for Co with spin up as the majority spin, there are many more spin down states than spin up states at the Fermi surface. According to the Pauli exclusion principle, only one electron can occupy a particular state. Therefore the spin down 4 s electrons experience a much stronger scattering giving rise to a considerable resistance at the N/F interface compared with the spin up 4 s electrons. As a result, the spin down electrons are more likely to be reflected due to the strong scattering while the spin up electrons are transmitted due to the lower scattering probability.

The first observation of a large GMR was reported independently by Fert and Grünberg in $\mathrm{Fe} / \mathrm{Cr}$ multilayers $[19,23]$. Figure 2.2 shows the experimental results for $\mathrm{Fe} / \mathrm{Cr}$ multilayers. The resistance is high when the alignment is antiparallel (AP) and is low when the alignment is parallel $(\mathrm{P})$.

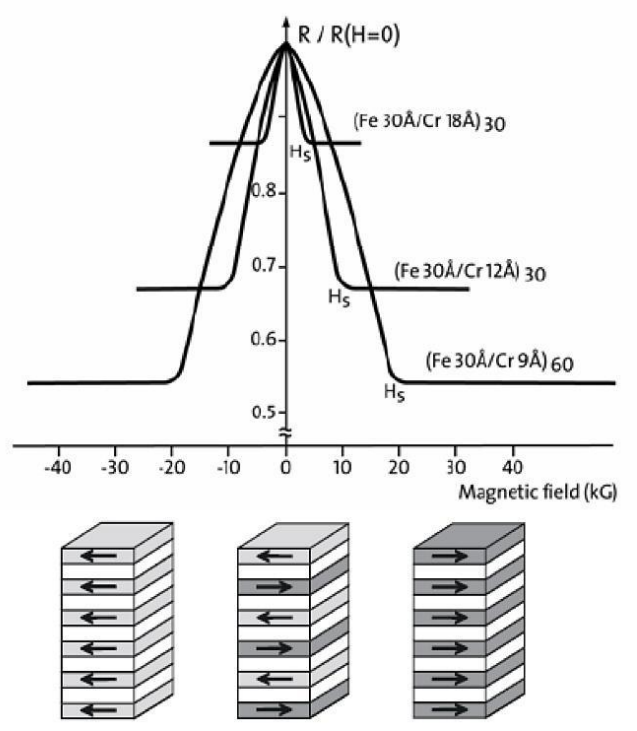

Figure 2.2 Resistance change due to an external magnetic field for Fe/Cr multilayers[19] 
The magnitude of the MR is expressed by the so-called MR ratio, defined as:

$$
M R=\frac{R_{A P}-R_{P}}{R_{P}}
$$

where $R_{A P}$ and $R_{P}$ are the resistance in $\mathrm{AP}$ and $\mathrm{P}$ alignment of the magnetization of the magnetic layers.

To understand the GMR effect, we consider the case where the current traverses a trilayer composed of a non-magnetic layer sandwiched by two ferromagnetic layers. Mott's two-current model is applicable to GMR in multilayers as a first approximation. In Chapter 5, I will discuss the fact that the spin-diffusion length also affects the GMR. For simplicity, here I will assume no spin-flipping occurs during the time the electron propagates through the trilayers. The two-current model is schematically described in Figure 2.3, where we ascribe two different resistances to each ferromagnetic layer in two cases: $R_{\uparrow}$ corresponds to those electrons that pass through the ferromagnet with the spin orientation parallel to the local magnetization, while $R_{\downarrow}$ means the spin orientation of the electrons is antiparallel to the local magnetization.

When the magnetization is in parallel alignment as shown in Figure 2.3(a),(b)\&(c), the spin up electrons experience less scattering and have a combined resistance of $2 \mathrm{R} \uparrow$, and the spin down electrons experience a total resistance of $2 R_{\downarrow}$, the total resistance in the parallel alignment is $R_{P}=2 R_{\uparrow} R_{\downarrow} /\left(R_{\uparrow}+R_{\downarrow}\right)$. Similarly, as shown in Figure 2.3 (d), (e)\&(f), the antiparallel alignment yields the total resistance of $R_{A P}=\left(R_{\uparrow}+R_{\downarrow}\right) / 2$. The difference in resistance between the two cases becomes: $\Delta \mathrm{R}=\mathrm{R}_{\mathrm{AP}}-\mathrm{R}_{\mathrm{P}}=(1 / 2)\left(\mathrm{R}_{\downarrow}-\mathrm{R}_{\uparrow}\right)^{2}$ 
$/\left(R_{\uparrow}+R_{\downarrow}\right)$. Thus the larger the difference between $R \uparrow$ and $R \downarrow$ the larger the magnetoresistance. This expression clearly shows that the magnetoresistance effect arises from the spin-dependent resistive behavior.

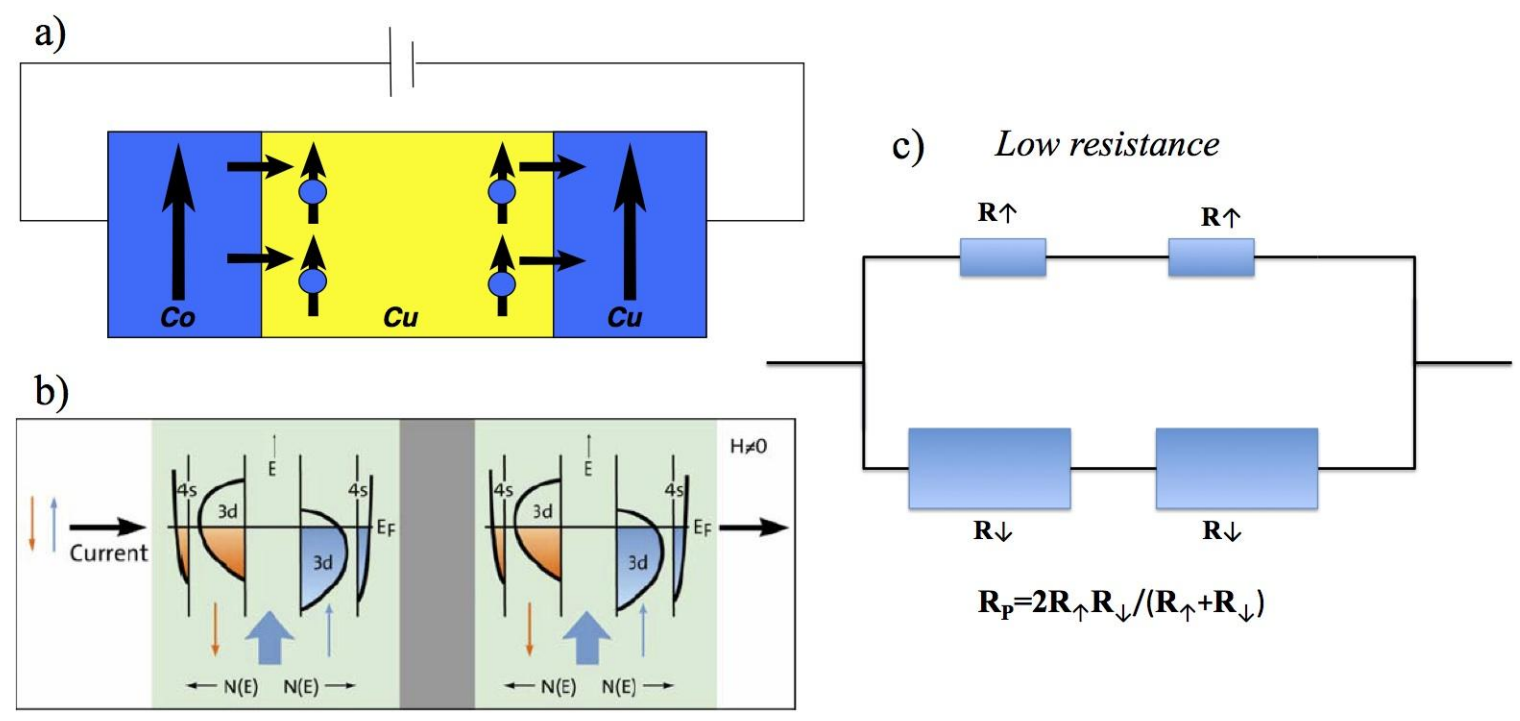

\section{d)}

e)
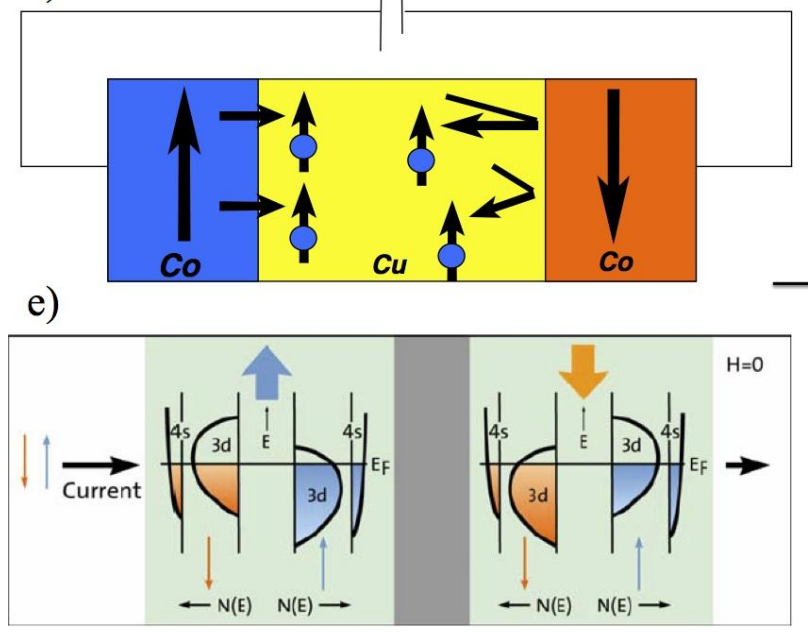

\section{f) High resistance}

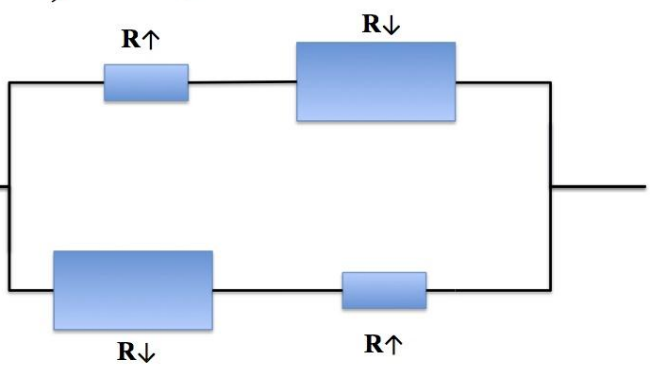

$$
\mathbf{R}_{\mathrm{AP}}=\left(\mathbf{R}_{\uparrow}+\mathbf{R}_{\downarrow}\right) / 2
$$

Figure 2.3 Mott's two-current model. (a) A schematic for the parallel (P) magnetization alignment. (b) Illustration of the electronic band structure for parallel alignment (the majority spin directions are indicated by two thick blue arrows at the bottom) (c) Equivalent circuit for the resistance of parallel alignment of magnetization. (d) A 
schematic for the antiparallel (AP) magnetization alignment. (e) Illustration of the electronic band structure for antiparallel alignment (the majority spin directions are indicated by the thick blue and orange arrows) (f) Equivalent circuit for the resistance of parallel alignment of magnetization. [Adapted based on Prof. Stu Wolf's lecture notes (UVa, 2013 Spring) and Ref.[21]]

\subsection{Spin-valve (SV) and pseudo spin-valve (PSV)}

Technological applications of GMR, for example, sensors, require a sharp response of the magnetization direction to the external magnetic field within a few Oe. To achieve such sensitivity, a four-layer structure is prepared with a tri-layer (F-N-F) and one of the ferromagnetic layers adjacent to an antiferromagnetic layer. This ferromagnetic layer is pinned by the antiferromagnetism and it is very hard to cause its magnetization direction to rotate, and only the other magnetic layer responds to the external magnetic field and its magnetization direction rotates in response to a small magnetic perturbation. This kind of four layer structure is called a spin-valve(SV) [24, 25], wheras the trilayer without a pinning layer is called a pseudo spin-valve(PSV).

Instead of employing different values of coercivity to distinguish between the two $\mathrm{F}$ layers as in pseudo spin-valves, as described above, the spin-valves have a fixed layer that is usually pinned in a particular direction through an effect called "exchange bias". By 
placing a ferromagnetic layer in contact with an antiferromagnetic (AF) layer, the ferromagnetic layer is biased in a direction defined by the antiferromagnet [26]. In Figure 2.4, it has been shown that the direction of this pinning can be defined by annealing the AF-F bilayer in the presence of a magnetic field above the Néel temperature of the antiferromagnet, the temperature above which the antiferromagnet becomes paramagnetic [27]. Upon cooling below the Néel temperature the antiferromagnet relaxes into its lowest energy state, which is determined by the magnetization direction of the F layer, resulting in a pinning of the $\mathrm{F}$ layer. Some of the various AF materials that are typically used for exchange biasing spin valves are FeMn, PtMn, and IrMn, all of which are chosen because of their high exchange bias field, larger than $150 \mathrm{Oe}$, and the stability of the exchange bias field to well above room temperature [28]. 


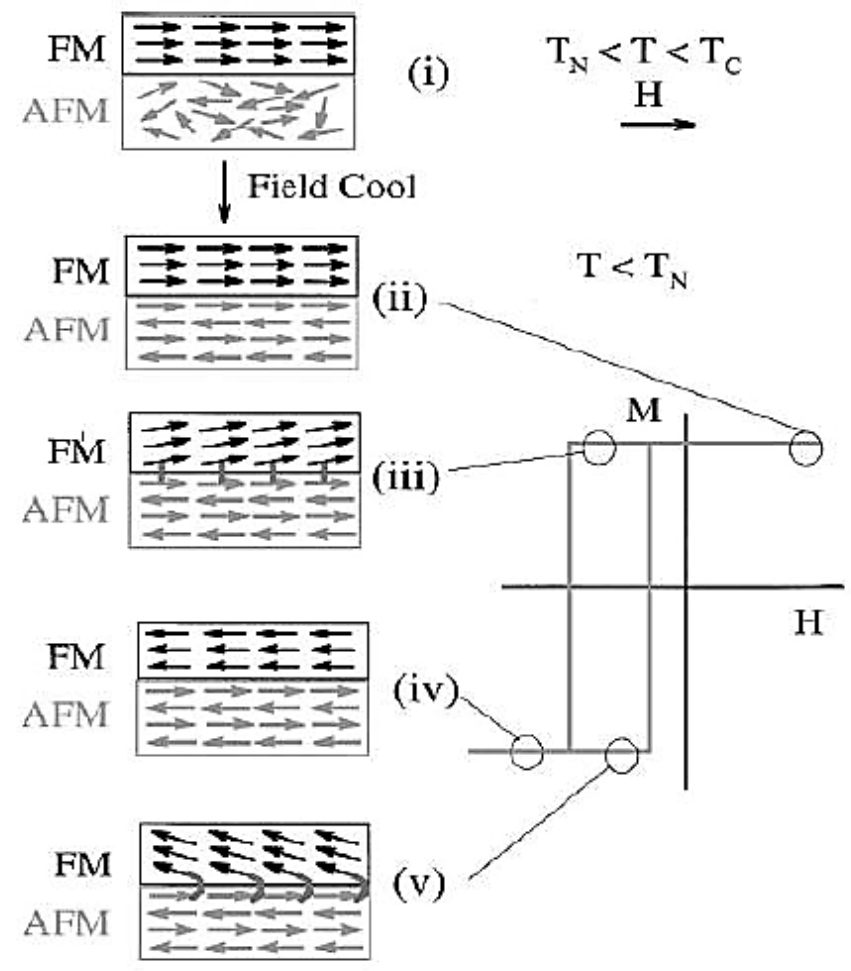

Figure 2.4 Schematic diagram showing the formation of exchange bias. (i)-(v) shows the spin configuration of an FM/AFM bilayer at different stage of an exchange biased hysteresis loop. Note that the spin configurations are just a simple cartoon to illustrate the effect of the coupling and they are not necessarily accurate portraits of the actual rotation of the FM or AFM magnetizations. (Adapted from Ref. [29])

\subsection{Tunneling magnetoresistance (TMR)}

The successful development of STT-MRAM relies on another important phenomenon in spintronics, the tunneling magnetoresistance(TMR). In a magnetic tunnel junction (MTJ), two conducting electrodes made of ferromagnetic materials are separated by a thin 
insulating tunnel barrier (for example, $\mathrm{MgO}$ or $\mathrm{Al}_{2} \mathrm{O}_{3}$ ). When a current passes through the tunnel barrier, the resistance depends on the relative orientation of the magnetizations of the two magnetic layers. In most cases, the resistance becomes higher when the magnetizations of the two magnetic electrodes are aligned antiparallel than that in parallel.

Thus the TMR ratio is defined as:

$$
T M R=\frac{G_{P}-G_{A P}}{G_{P}}=\frac{R_{A P}-R_{P}}{R_{P}}
$$

where $G_{P}$ and $G_{A P}$ are the conductance when the two ferromagnetic materials have their magnetizations parallel and antiparallel, and $R_{P}$ and $R_{A P}$ are the resistances in the parallel and antiparallel states, respectively.

(a)
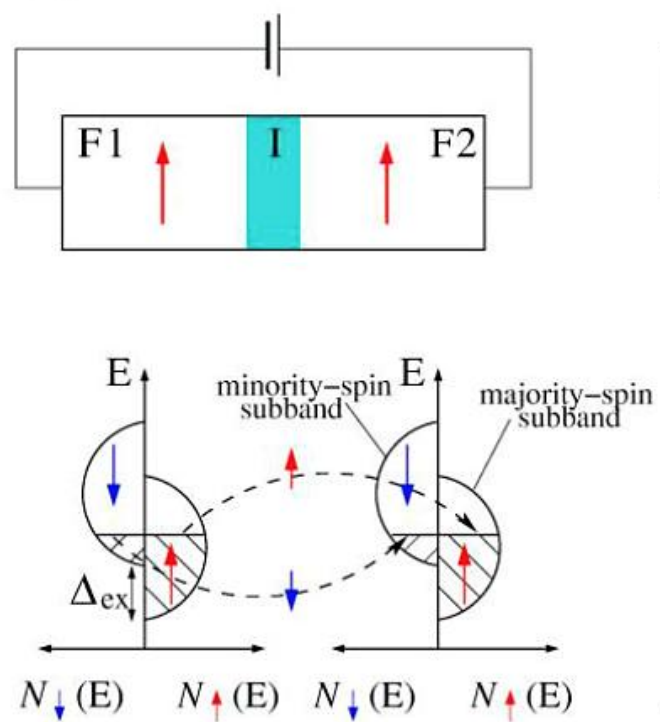

(b)
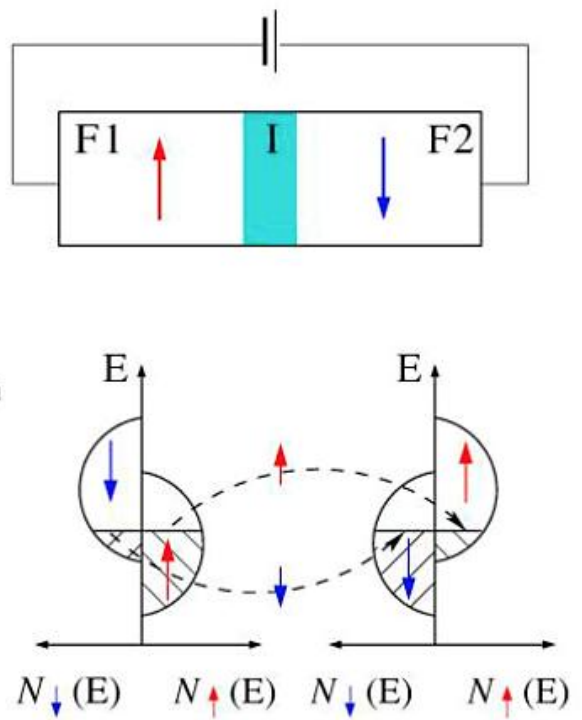

Figure 2.5 Schematic of the spin-dependent tunneling process for (a) parallel and (b) anti-parallel states, according to Jullière's model. Dashed lines indicate the spin-conserved tunneling. (Reproduced from Ref.[29]) 
The origin of TMR can be explained in terms of Julière's model by considering the difference in the density of states at the Fermi level for spin up and spin down electrons [30]. Figure 2.5 shows a schematic representation of the spin-dependent tunneling process for the two different magnetization configurations: parallel (P) and antiparallel (AP). At each configuration the majority spin state is energetically lowered due to exchange splitting. This ends up with a different density of states at the Fermi level for spin up and spin down electrons. As the spin-polarized electrons tunneling, the spin is known to be conserved during tunneling [30]. In the tunneling process, it is assumed that tunneling conductance is proportional to the product of the density of states of the two ferromagnetic layers. Then the conductance for the parallel and antiparallel configurations can be expressed as $[30,31]$ :

$$
\begin{gathered}
G_{P} \propto n_{\uparrow}(1) \cdot n_{\uparrow}(2)+n_{\downarrow}(1) \cdot n_{\downarrow}(2)=\frac{1+P_{1} P_{2}}{2} \\
G_{A P} \propto n_{\uparrow}(1) \cdot n_{\downarrow}(2)+n_{\downarrow}(1) \cdot n_{\uparrow}(2)=\frac{1-P_{1} P_{2}}{2}
\end{gathered}
$$

where $n_{\uparrow}(1$ or 2$)$ and $n_{\downarrow}(1$ or 2$)$ represent the density of states for spin-up and spin-down electrons at the Fermi energy level of the electrode 1 or 2 , respectively. $\mathrm{P}_{1}$ and $\mathrm{P}_{2}$ in the above equation are the spin-polarization factors of the two electrodes and defined as:

$$
\mathrm{P}=\frac{\mathrm{n} \uparrow-\mathrm{n} \downarrow}{\mathrm{n} \uparrow+\mathrm{n} \downarrow}
$$


By combining equation (2-3), (2-4), (2-5) and (2-6), the TMR ratio can be written as:

$$
T M R=\frac{2 P_{1} P_{2}}{1-P_{1} P_{2}}
$$

In principle, the TMR ratio becomes infinitely large when electrodes with $100 \%$ spin-polarization factor are used.

\subsection{Spin transfer torque (STT)}

While the GMR and TMR effect serve as the basis for spintronics, it is the spin-transfer effect that opens up the new era of nano-scale devices for memory and other electronic applications. Slonczewski and Berger first predicted that spin polarized DC currents passing through a spin-valve or MTJ multilayer device can transport angular momentum from one magnetic layer to another, thereby exerting a torque on the local magnetization $[32,33]$. Figure 2.6 shows a schematic diagram for the spin-transfer effect. When the electrons flowing through a SV or MTJ nano-magnet, the electrons with random spin-orientation at the beginning will get spin-polarized along the local magnetization direction of the reference layer or the pinned layer. As the electrons move on and pass through the free layer, the electrons' spin orientation rotates toward the direction of the free layer's magnetization. Examining the initial and finial angular momentum for the electron, there is a change of the angular momentum, which results into a torque. According to the angular momentum conservation, the same amount of torque must be exerted on the free 
layer's magnetic moments in return, which is called the spin-transfer torque.
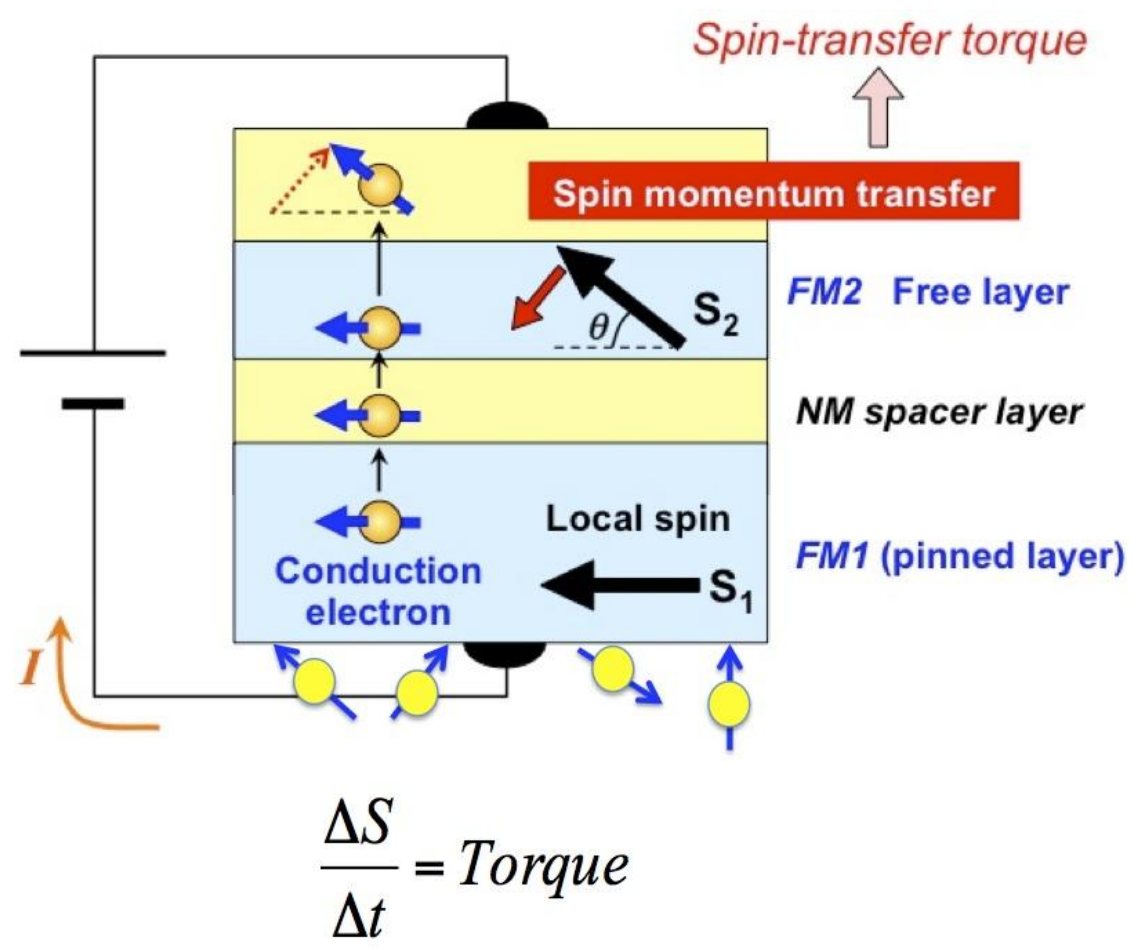

Figure 2.6 Schematic showing electrons being spin-polarized along $\vec{S}_{1}$ by the bottom magnetic layer and, in the act of rotating toward the new polarization direction $\vec{S}_{2}$ in the free layer, transferring spin momentum to the free layer magnetic moment. If this torque is large enough it can overcome the damping and switch the magnetization of the free layer, or it can balance out the damping and leads to stable precession. 


\subsubsection{The model for spin transfer-Slonczewski model}

Slonczewski (1996) and Berger (1996) predicted that electron currents in magnetic multilayer devices could transport angular momentum from one magnetic layer to another, therefore exerting a torque on the local magnetization [33, 34]. In their model, they assume ballistic electron transport and use the WKB approximation for electron transport. This torque is quantified and is then inserted into the Landau-Lifshitz-Gilbert (LLG) equation as an additive term. I will briefly describe the derivation of this spin transfer torque, as originally outlined by Slonczewski, for the simplest ideal magnetic trilayer structure [33, $35]$.

Figure 2.7 shows the schematic of the spin transfer system used in the Slonczewski model. The system is composed of five metallic layers, $\mathrm{A} / \mathrm{F}_{1} / \mathrm{B} / \mathrm{F}_{2} / \mathrm{C}$. The vectors $\hbar \overrightarrow{S_{1}}$ and $\hbar \overrightarrow{S_{2}}$ are the total spin momentum per unit area of the ferromagnets $F_{1}$ and $F_{2}$, with $\theta$ being the angle between $\overrightarrow{S_{1}}$ and $\overrightarrow{S_{2}}$. The spin torque is generated when a current flows through the stack. 


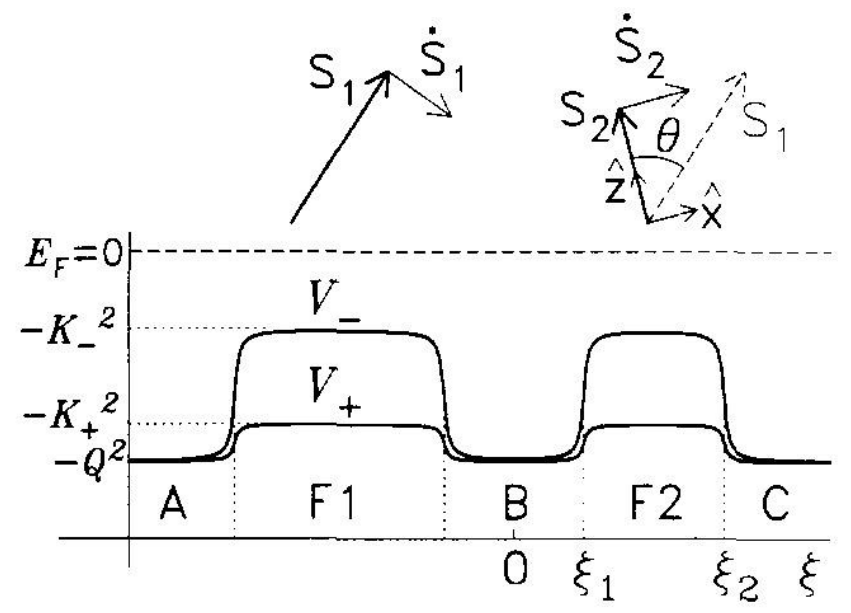

Figure 2.7 Simple illustration of the five-layer spin transfer system proposed by Slonczewski.(Adapted from Ref.[33])

Now we treat $F_{1}$ as a polarizer and consider the three-layer system of $B / F_{2} / C$ in the case where the incident electron has its spin along $\overrightarrow{S_{1}}$. We translate the vectors into the reference frame where $\overrightarrow{S_{2}}=S_{2} \hat{z}$ is the axis of spin quantization in this frame, and $\left(\overrightarrow{S_{1}} \times \overrightarrow{S_{2}}\right) /\left(S_{1} S_{2}\right)$ is in the $\hat{y}$ direction. By transforming the incident electron state into the reference frame, $\overrightarrow{S_{1}}$ can be expressed as $(\cos (\theta / 2), \sin (\theta / 2))$, where $\cos (\theta / 2)$ is the spin up component in the spin-quantization frame, and $\sin (\theta / 2)$ is the spin down component. The exchange interaction gives the potential $V \pm(\xi)$ where $\xi$ is the position coordinate that is perpendicular to the plane of the multilayer, and the " \pm " corresponds to the majority/minority spin bands. $k \pm$ corresponds to the wave vector with the energy at $V \pm$ in Figure 2.7.

In the free electron approximation, given an electron with energy $E$ we can describe 
the wave vector along $\xi$ in terms of $E, V \pm(\xi)$, and $k_{p}$, where $\overrightarrow{k_{p}}$ is the wave vector perpendicular to axis $\xi$. Employing a unit system in which $\hbar^{2} /\left(2 m_{e}^{2}\right)$ is unity, these wave numbers are given by the formula:

$$
k_{ \pm}=\left(E-k_{p}^{2}-V_{ \pm}\right)^{1 / 2}
$$

In the WKB approximation the wave function of an electron is:

$$
\psi(x)=\frac{A}{k^{1 / 2}} \mathrm{e}^{\mathrm{i} \int k(x) d x}
$$

where $k$ is the wave vector and " $\mathrm{A}$ " is a constant. Incorporating the electron's wave function and the spin momentum matrix, for the tri-layer system, the wave function becomes:

$\psi(x)$

$=\left(k_{+}^{-\frac{1}{2}}(\xi) \exp \left(i \oint_{0}^{\xi} d \xi^{\prime} k_{+}\left(\xi^{\prime}\right)\right) \cos \left(\frac{\theta}{2}\right), k_{-}^{-\frac{1}{2}}(\xi) \exp \left(i \oint_{0}^{\xi} d \xi^{\prime} k_{-}\left(\xi^{\prime}\right)\right) \sin \left(\frac{\theta}{2}\right)\right)$

Using this wave function we can define the Pauli-spin flux as[33]:

$$
\begin{gathered}
\phi_{+}(\xi)=\phi_{x}+i \phi_{y}=i\left(\frac{d \psi_{+}^{*}}{d \xi} \psi_{-}^{*}+\psi_{+}^{*} \frac{d \psi_{-}^{*}}{d \xi}\right) \\
\phi_{\mathrm{z}}(\xi)=\operatorname{Im}\left(\psi_{+}^{*} \frac{d \psi_{+}}{d \xi} \pm \psi_{-}^{*} \frac{d \psi_{-}}{d \xi}\right)
\end{gathered}
$$

Within regions $\mathrm{B}$ and $\mathrm{C}$, for the state with wave function of (2-10) the Pauli-spin flux approaches:

$$
\phi_{+}(\xi)=\exp \left(i \int_{0}^{\xi}\left(k_{-}-k_{+}\right) d \xi^{\prime}\right) \sin (\theta), \phi_{\mathrm{z}}=\cos \theta
$$


Now we consider the conservation of angular momentum as the electrons pass through the second magnet. Any angular momentum that the electrons lose will be transferred to $F_{2}$. $\Delta \mathrm{S}_{2}$ is the sum of the inward spin fluxes from $\mathrm{B} \& \mathrm{C}$ sides of the layer $\mathrm{F}_{2}$. So:

$$
\begin{aligned}
\Delta S_{2, x}+\mathrm{i} \Delta S_{2, y} & =\frac{\phi_{+}(0)-\phi_{+}(+\infty)}{2} \\
& =\frac{1}{2}\left(1-\exp \left(i \int_{0}^{\xi}\left(k_{-}-k_{+}\right) d \xi^{\prime}\right)\right) \sin (\theta)
\end{aligned}
$$

For the $z$ component: $\Delta S_{2, z}=0$

The mean of the spin transfer averaged with respect to the direction of electron motion is given by Eq. (2-14):

$$
\left\langle\Delta S_{2}\right\rangle=(\sin (\theta), 0,0) / 2
$$

This simply means the total absorption of the transverse $(x)$ spin component by $\mathrm{F}_{2}$ layer.

Extending this simple model to include the whole five-layer system, the spin transfer torque is expressed:

$$
d \vec{S}_{1,2} / d t=(J g / e) \hat{S}_{1,2} \times\left(\hat{s}_{1} \times \hat{S}_{2}\right)
$$

where $J$ is the current density, $\hat{s}_{1,2}$ are unit vectors $\hat{s}_{\mathrm{i}}=\overrightarrow{\mathrm{s}_{l}}, \frac{\mathrm{s}_{\mathrm{i}}}{}$, and $g$ is the scalar factor defined as $[33,36]$ :

$$
g(\theta, P)=\frac{P \Lambda^{2}}{\left(\Lambda^{2}+1\right)+\left(\Lambda^{2}-1\right) \cos \theta}, \Lambda^{2}=G R_{A P}
$$

where $P$ is the spin polarization in the $\mathrm{F}$ layers and $G$ is the conductance. 


\subsubsection{The critical current density $\left(J_{c}\right)$ for magnetization reversal}

To understand how the spin transfer torque affects the magnetic moment, the Slonczewski term Eq. (2-16) was included in the Landau-Lifshitz-Gilbert (LLG) equation, the equation that describes the spin dynamics. For simplicity, I will first consider a system with only uniaxial anisotropy $K_{u}$ where the anisotropy gives an angle dependent energy to the nano-magnet of the form $E=K_{u} \sin ^{2} \theta=\frac{H_{u} M_{s}}{2} \sin ^{2} \theta, K_{u}$ is the uniaxial anisotropy constant, $M_{s}$ the saturation magnetization and $H_{u}$ is the uniaxial anisotropy field, $\theta$ is the angle between the magnetization and the anisotropy axis. A fixed frame is defined by orthogonal unit vectors $\hat{a}, \hat{b}, \hat{c}$, of which $\hat{c}$ is set to the uniaxial anisotropy direction. Assume that the magnet behaves as a single domain, this gives:

$$
d \overrightarrow{S_{2}} / d t=\overrightarrow{S_{2}} \times\left\{\gamma H_{U}\left(\hat{c} \cdot \hat{S}_{2}\right) \hat{c}-\alpha \frac{d \overrightarrow{S_{2}}}{d t}-\frac{J g(\theta, P)}{e S}\left(\hat{s}_{1} \times \hat{S}_{2}\right)\right\}
$$

Here $\alpha$ is the Gilbert damping constant. In the absence of the spin-transfer term and damping, the solution of this equation is elliptical precession of about the $c$-axis with $\overrightarrow{S_{2}}=(A \sin \theta \cos (\omega t), A \sin \theta \sin (\omega t), \cos (\theta))$. The amplitude 'A' will be decaying with time when include the damping term, while the spin transfer term can amplify or attenuate the amplitude depending on the current direction.

For small amplitude precession around $\theta=0$, assume $S_{2}$ has the easy axis directions along $\pm \hat{c}$, the averaged rate of change for the total energy is [37]:

$$
\left.\left\langle\frac{d E}{d t}\right\rangle \approx \kappa H_{u} \cos (\theta)\left\{-\alpha \gamma H_{u} \cos (\theta)+\frac{J g(\theta, P)}{S e}\right)\right\}
$$


Where $\kappa$ is a coefficient, for small angle $\theta \cong 0$, if $\left\langle\frac{d E}{d t}\right\rangle=0$, then $\mathrm{F}_{2}$ will be in a stable state since perturbation or precession at this condition will return to the low energy state of $\theta=0$. And if the current is ramped up, the system will remain stable until the critical current density is reached, in other words, the system can switch from parallel $(\theta=0)$ to antiparallel $(\theta=\pi)$ when:

$$
J>J_{C}^{P-A P}=\frac{\alpha \gamma H_{u} e S}{g(0, P)}
$$

To be more general, we can rewrite the above equation in terms of the critical current $\left(I_{c}\right)$, volume $(V)$ and saturation magnetization $(M s)$ :

$$
I>I_{C}^{P-\mathrm{A} P}=\frac{\alpha \gamma H_{U} M_{s} V}{g(0, P)}
$$

By similar argument, antiparallel $(\theta=\pi)$ to parallel alignment when:

$$
I>I_{C}^{A P-P}=\alpha \gamma H_{u} M_{s} V / g(\pi, P)
$$

In the case for $\mathrm{Ni}_{80} \mathrm{Fe}_{20} / \mathrm{Cu} / \mathrm{Co}_{90} \mathrm{Fe}_{10}$ structure that will be discussed in Chapter 7, when electrons are passing from the reference layer $\left(\mathrm{Co}_{90} \mathrm{Fe}_{10}\right)$ to the free layer $\left(\mathrm{Ni}_{80} \mathrm{Fe}_{20}\right)$, the electrons first get spin-polarized along $\vec{m}_{\mathrm{COFe}}$ (spin-up for example), the spin-up electrons then exert a torque on $\vec{m}_{N i F e}$. Once that torque is big enough to overcome the damping, the $\vec{m}_{N i F e}$ switches into the parallel state. This current density is defined as the critical current density, $J_{C}^{A P-P}$. In the parallel to antiparallel transition, when electrons are injected from the $\mathrm{Ni}_{80} \mathrm{Fe}_{20}$ layer to the $\mathrm{Co}_{90} \mathrm{Fe}_{10}$ layer, the spin down electrons from $\vec{m}_{\text {NiFe }}$ reflect off of the $\mathrm{Ni}_{80} \mathrm{Fe}_{20} / \mathrm{Cu}$ interface and transfer the angular momentum to $\vec{m}_{\text {CoFe }}$. 
These spin down electrons exert a torque that switch the nano-magnet into the antiparallel state when the critical current density is reached, $J_{C}^{P-A P}$.

More quantitative calculations had been carried out based on the Landau-Lifshitz-Gilbert-Slonczewski equations to take into account of not only the anisotropy fields but also the demagnetizing field $2 \pi M_{S}$, dipolar field $H_{d i p}$, and external fields $[36,38,39]$. For in-plane magnetized devices, the critical currents for reversal from parallel to antiparallel (P-AP) and antiparallel to parallel (AP-P) are given, respectively, by:

$$
\begin{gathered}
I_{C}^{P-A P} \approx \frac{A \alpha M_{S} V}{g(0, P)}\left(H+H_{d i p}+H_{K \|}+2 \pi M_{S}\right) \\
I_{C}^{A P-P} \approx \frac{A \alpha M_{S} V}{g(\pi, P)}\left(H+H_{d i p}-\left(H_{K \|}+2 \pi M_{S}\right)\right)
\end{gathered}
$$

where $M_{S}, V$ and $\alpha$ are the saturation magnetization, volume and Gilbert damping constant for the free layer, respectively, and $P$ is the spin polarization of the current. The scalar factor $g$ depends on the relative angle of the reference-layer and free-layer magnetizations, and spin polarization $P$. The factor $A$ is a constant coefficient of the order of $10^{11} \mathrm{mAOe}^{-1}$ $\mathrm{emu}^{-1} . H, H_{d i p}$ and $H_{K / /}$ are the in-plane applied field, the dipole field from the reference layer acting on the free layer and the uniaxial in-plane anisotropy field, respectively. The factor $2 \pi M_{S}$ arises from the demagnetizing field of the thin-film geometry. For the out-of-plane case, the critical currents are given by [38]:

$$
I_{C}^{P-A P} \approx \frac{A \alpha M_{S} V}{g(0, P)}\left(-H-H_{d i p}+H_{K \perp}-4 \pi M_{S}\right)
$$




$$
I_{C}^{A P-P} \approx \frac{A \alpha M_{S} V}{g(\pi, P)}\left(-H-H_{d i p}-\left(H_{K \perp}-4 \pi M_{S}\right)\right)
$$

where $H_{K \perp}$ is the perpendicular uniaxial anisotropy field of the free layer, and $H$ and $H_{d i p}$ are now fields perpendicular to the film. In this geometry the demagnetizing field is collinear to the anisotropy field giving an effective perpendicular anisotropy field $H_{K}=$ $H_{K \perp}-4 \pi M_{S}$ where $H_{K}$ must be greater than zero for perpendicular anisotropy. 


\subsubsection{Application of STT: STT-MRAM}

The spin-transfer torque can switch the magnetization of the free layer, which was predicted theoretically $[32,33]$ and was then experimentally demonstrated in a $\mathrm{Co} / \mathrm{Cu} / \mathrm{Co}$ nano-pillar with in-plane magnetization $[4,40]$. Figure 2.8 shows the hysteretic switching of magnetization by DC currents in nanopillars demonstrated in Cornell University and Grandis Inc.

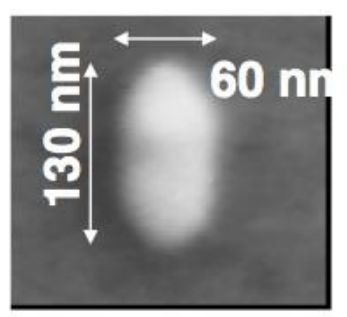

Cornell, 2000

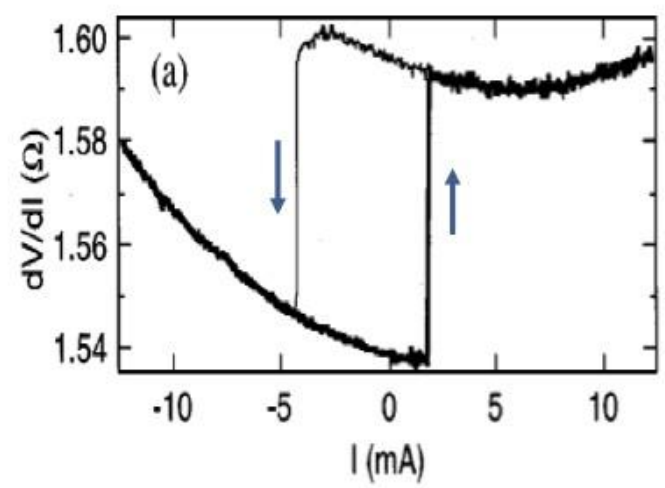

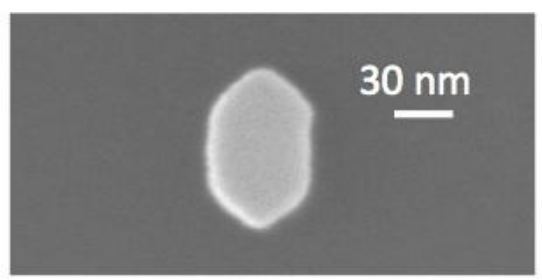

Grandis, 2004

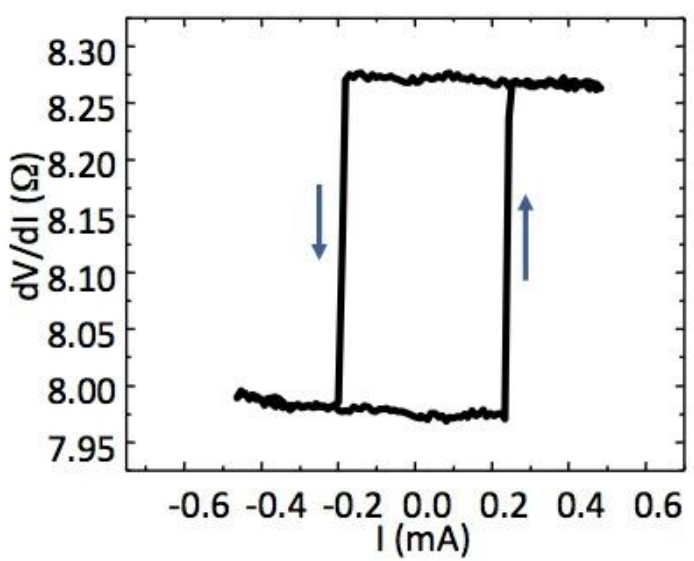

Figure 2.8 Typical DC current induced magnetization switching in magnetic multilayer nanopillars. The size of the pillar is shown in the SEM images. (Adapted from Prof. Stu Wolf's lecture notes (UVa, 2013 Spring)) 
The theoretical prediction of the critical current density for switching in in-plane magnetic devices was expressed in (2-25) and (2-16) and can be combined as:

$$
I_{C} \approx \frac{A \alpha M_{S} V}{P g(\theta)}\left(H+H_{d i p} \pm\left(H_{K}+2 \pi M_{S}\right)\right)
$$

The " \pm " sign depends on the electron flow direction.

Many efforts have been carried out to reduce the critical current density of the magnetization switching based on Eq. (2-27). For example, reducing $M_{S}$ by using $\mathrm{CoFeB}$ as the free layer [41]; increasing $P$ by using a double spin-filter structure [42], a synthetic antiferromagnetic pinning structure [43], inserting a Ru spin scattering layer [44]; or by reducing the damping constant by rare-earth doping [45]. Another approach is to introduce inhomogeneity into the nano-magnets and to achieve the reversal by domain wall propagation $[46,47]$.

It is noteworthy to point out that multilayers incorporating perpendicular magnetic anisotropy (PMA) electrodes are of special interest from the application point of view. The energy barrier of the bit, such as in a hard disk drive (HDD) recording cell, against thermal fluctuation of the magnetization is $M_{S} V H_{K} / 2$, where $H_{K}=H_{K \perp}-4 \pi M_{S}$ for perpendicular anisotropy devices. Thus, one potential advantage to the PMA device is that the critical currents for switching (for small $H$ and $H_{d i p}$ ) the magnetization is directly proportional to the anisotropy, hence, the stability of the bit. However, according to Eq. (2-27), for the in-plane devices, the current must overcome the additional demagnetizing factor $2 \pi M_{S}$ that does not contribute to the stability of the bit against thermal fluctuations, 
but suppresses spin-torque induced switching.

STT-MRAM evolved from the Magnetic-resistive Random Access Memory (MRAM). MRAM is a non-volatile memory that could be powered off and on very rapidly without loss of information. It is a big step forward to get rid of standby power [48]. Conventional MRAM [as shown in Figure 2.9(a)] has several limitations, for example, creating high enough magnetic fields requires very high currents and writing power. Secondly, with miniaturization one must increase the anisotropy field to increase the thermal stability $\left(K_{U} V / k_{B} T\right)$, while this is at the cost of increasing the switching magnetic fields, hence the switching currents. Moreover, the crosstalk between adjacent writing lines decreases the writing reliability. The development of spin-transfer torque MRAM (STT-MRAM) overcomes those limitations. A schematic diagram of an STT-MRAM is shown in Figure 2.9(b). The word line is connected to the gate of a transistor which is used to select the MTJ to be written or read. The writing is highly selective and is done by applying either a positive or negative spin-polarized current between the bit line and source line. The reading is done by applying a weaker current to the bit line to sense the resistance of the MTJ. The architecture no longer requires a write line to create a magnetic field, so it is highly scalable. Besides, STT switching depends on current density rather than the current itself, hence, the writing currents scales down with cell size. Many studies on the prospects for scaling down have been carried out [49, 50]. Further improvements in the STT-MRAM can be achieved by bringing down the switching current density below $5 \times 10^{5} \mathrm{~A} / \mathrm{cm}^{2}$ and to 
decrease the write time below approximately $5 \mathrm{~ns}$ [51].
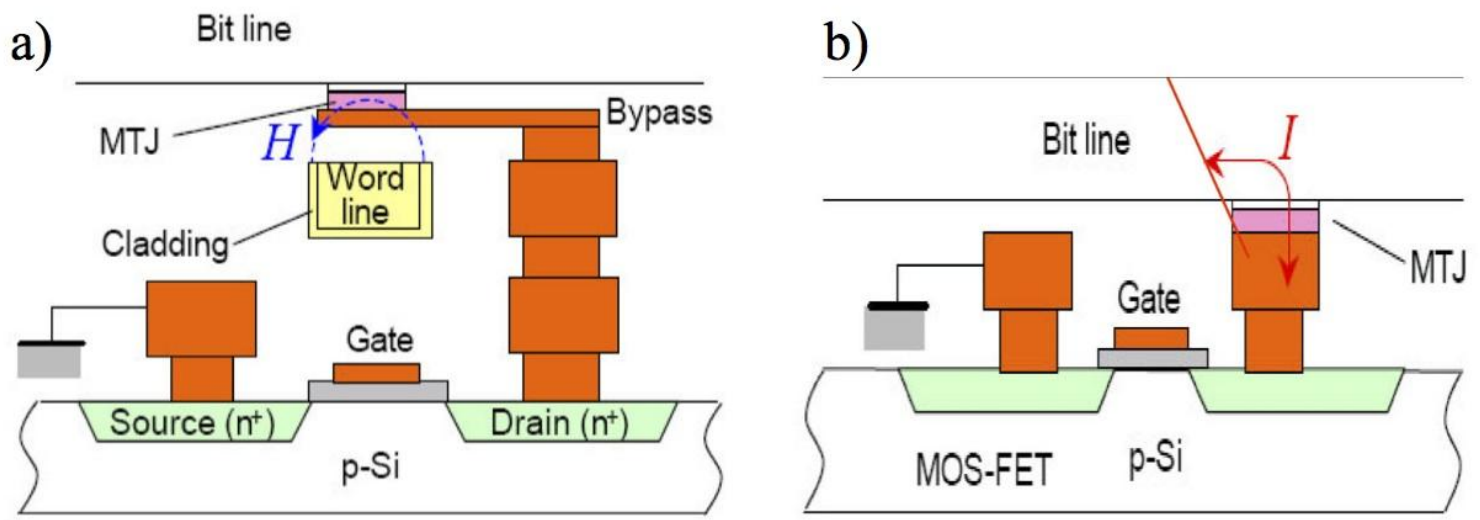

Figure 2.9 (a) Schematic diagram of MRAM using MTJ. (b) Schematic diagram of STT-MRAM. [Adapted from Prof. Stu Wolf's lecture notes (UVa, 2013 Spring)]

\subsubsection{Application of STT: Spin torque nano-oscillator (STNO)}

On the other hand, spin-transfer torque can excite precession of the free layer magnetic moment leading to sustained oscillation at microwave frequencies once the STT balances out the damping $[4,5,52]$. The first complete observation of microwave emission from magnetic nano-pillars with in-plane magnetization was performed by Kiselev et al. in 2003 [4]. They used a $\mathrm{Co} / \mathrm{Cu} / \mathrm{Co}$ GMR nano-pillar with a size of $\sim 130 \times 70 \mathrm{~nm}^{2}$. As shown in Figure 2.10(a), for fields smaller than the coercive field of the free layer, the spin-polarized currents produce hysteretic switching between the parallel and antiparallel states. While for field larger than 600 Oe, the current produced peaks in the differential resistance, which was associated with the spin torque oscillation. During the oscillation, the STT drove the 
free layer magnetic moment into a cyclic trajectory with frequency typically in the $\mathrm{GHz}$ range. Because of the GMR effect, the resistance of the pillar also oscillates with the continuous precession of the free layer magnetic moments. The oscillation was detected by measuring the power spectrum using a bias-tee and amplifier circuit. The observed spectra taken under an external field of -2kOe are shown in Figure 2.10(b). For current up to 2.4 $\mathrm{mA}$, the spectrum intensity was almost unchanged. The peak frequency matched with the FMR (ferromagnetic resonance) frequency of the free layer, which can be deducted from the Kittel equation [53]. A further increase of the currents results in a strong increase in the peak height and lowering of the peak frequency (red shift).
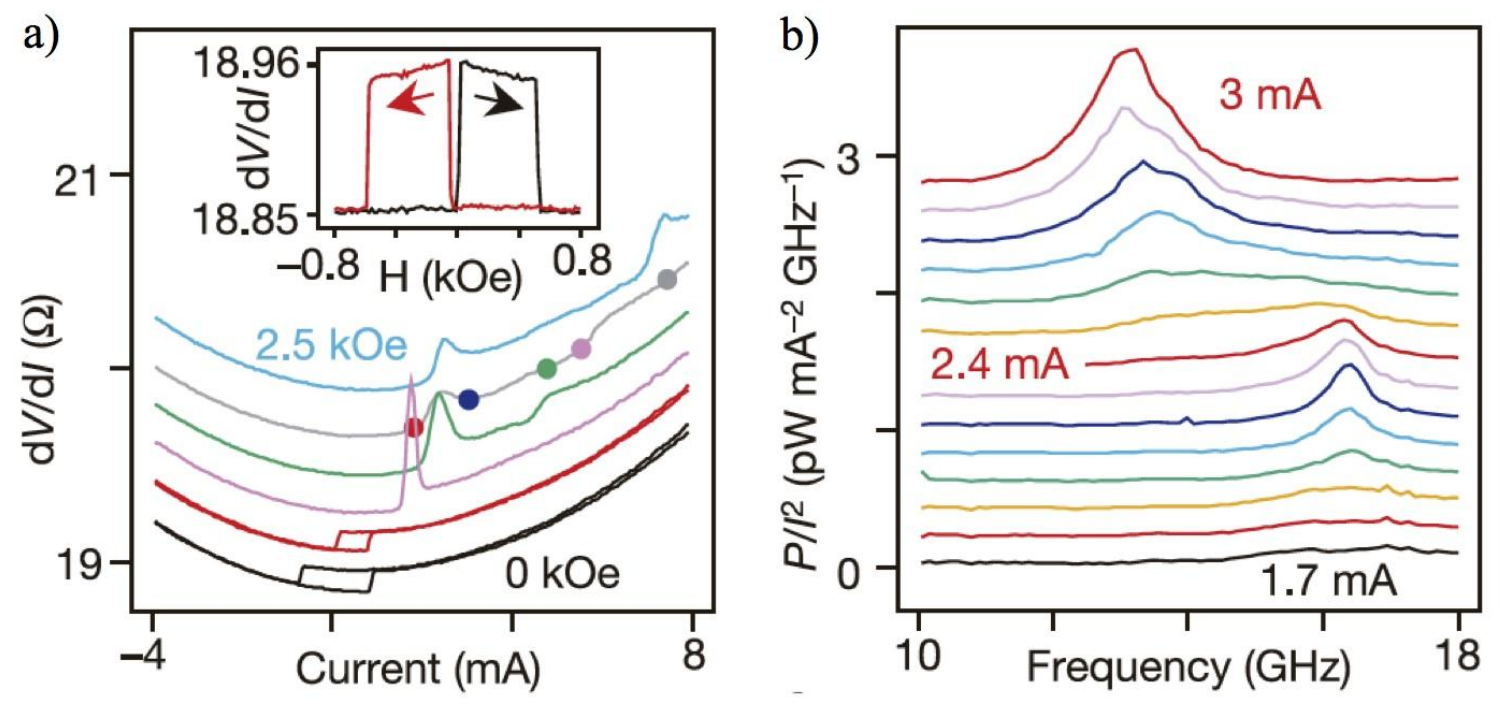

Figure 2.10 (a) Differential resistance versus current at different magnetic fields of O(bottom), $0.5,1.0,1.5,2.0$ and $2.5 \mathrm{kOe}$. (b) RF power density spectra normalized by square of the injected current. The emission was observed for a GMR nano-pillar with in-plane magnetization under $-2 \mathrm{kOe}$ of the external field. (Adapted from Ref. [4]) 
The dynamic stability diagram of the nano-pillar with in-plane magnetization obtained by Kiselev et al. is illustrated in Figure 2.11. For a zero external field and zero current the system is in the bistable state (P/AP in the figure). The application of a positive (negative) current causes the free layer magnetization to undergo a transition from the $\mathrm{P}(\mathrm{AP})$ to $\mathrm{AP}(\mathrm{P})$ state and stabilizes the AP (P) state [dotted line (i)]. The system shows hysteresis along the line (i). For zero current, if we apply a negative (positive, not shown in figure) external field, the system switches to the P (AP) state [along dotted line (ii)]. The system again shows hysteresis along the line (ii). For fields larger than $\sim 1 \mathrm{kOe}$, the switching does not occur, instead, the S, L, W correspond to the oscillation regimes. With a further increase of current, the precession changes orbits from small angle oscillation to large angle oscillation (regime S) and then to an out-of-plane oscillation regime L [along dotted line (iii)]. When an intermediate negative field and a large positive current were applied, a new phase "W" (shown in Figure 2.11) appeared. The very wide spectra observed in region $\mathrm{W}$ were attributed to a chaotic motion of the spins [4]. The overall dynamic diagram was well explained by micromagnetic simulations including the region "W" [54]. It was shown that vortex generation and annihilation were the main origin of the chaotic behaviour in "W" [54]. 


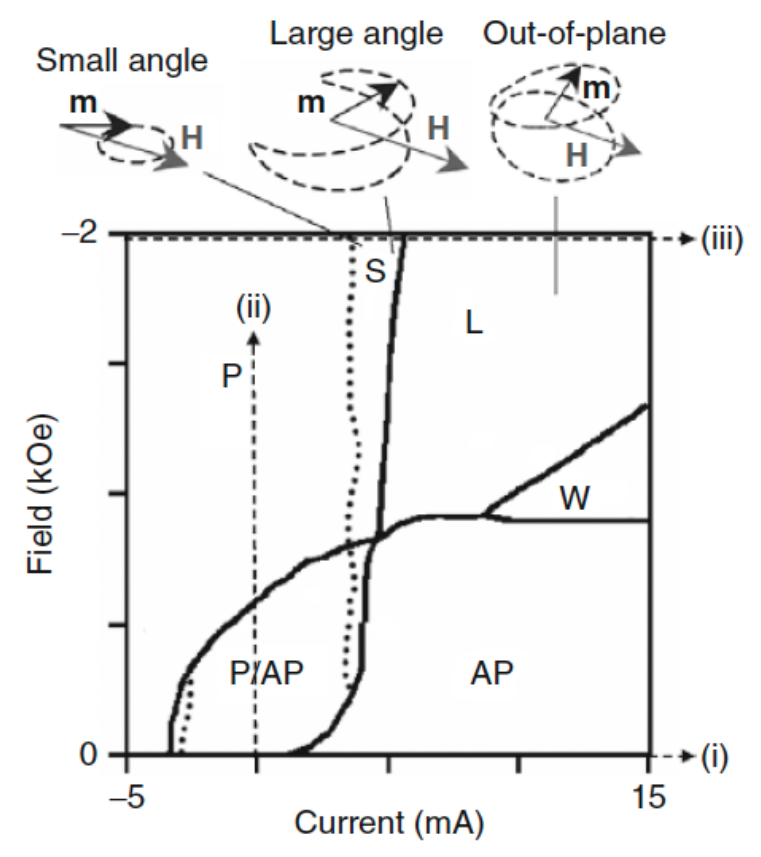

Figure 2.11 Dynamic stability diagram obtained from the $\mathrm{Co} / \mathrm{Cu} / \mathrm{Co}$ in-plane nano-pillars. (Adapted from Ref. [4])

Apart from in-plane magnetic nano-pillars, the spin torque oscillation has also been observed in all-perpendicular nano-pillars with both reference layer and free layer having perpendicular anisotropy[38] and in magnetic hybrid nano-pillars [17, 55]. Rippard et al. have also demonstrated spin torque oscillations in nano-contacts [5, 56]. A schematic structure of the nano-contact is shown in Figure 2.12. 


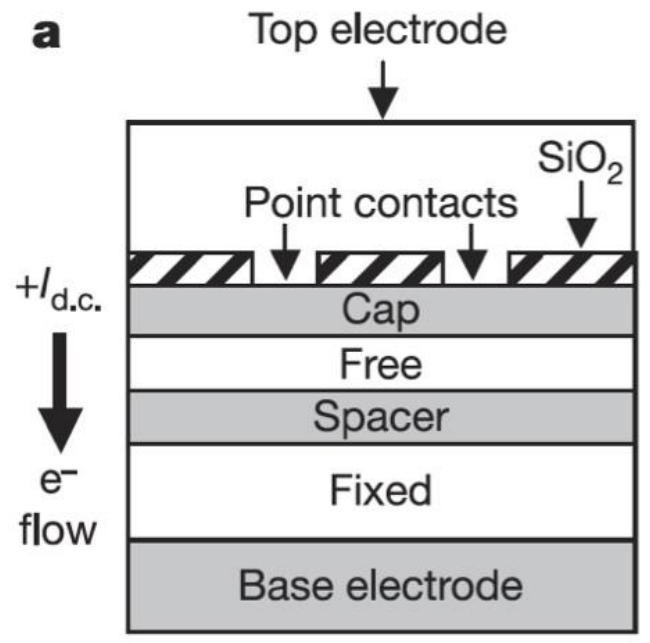

b Points contacts

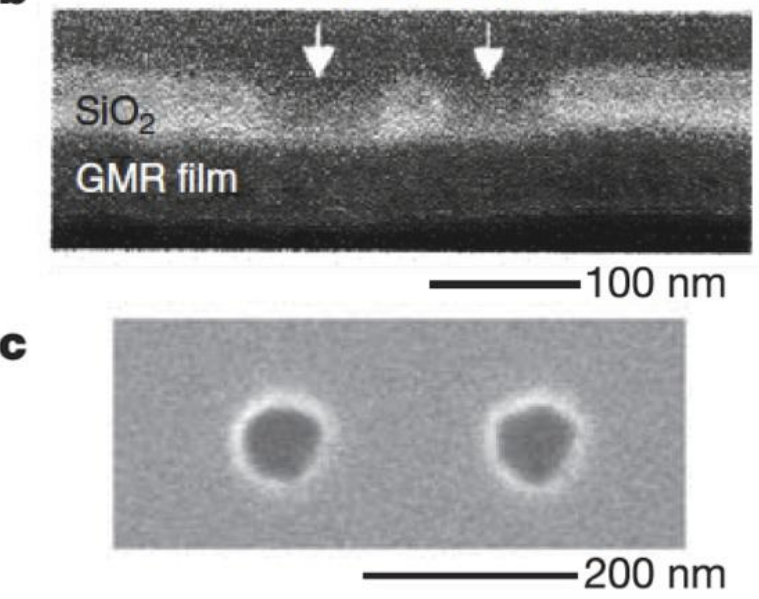

Figure 2.12 (a) Schematic cross section of the magnetic point contact. (b) Cross-sectional SEM image of the two 80-nm-diameter contacts that were used to observe mutual coupling of the STO. (c) SEM top image of the coupled point contacts. (Adapted from Ref.[57])

To date, the largest output power obtained in STNO structures is $\sim 0.5 \mu \mathrm{W}[58]$ — far too low for applications, such as resonators, nano-scale transmitters and receivers, signal mixers, signal amplifiers and so on, which require levels at least in the $\mu \mathrm{W}$ range [59]. According to Ref. [4], [58] and [60], the output power of the spin torque oscillation in the nano-pillar with in-plane magnetization can be expressed as:

$$
\begin{gathered}
P_{\text {Output }} \cong \eta\left(M R^{\prime}\right)^{2} \frac{R\left(\theta_{0}\right) J^{2}}{8}\left(\frac{\theta_{\text {max }}}{2}\right)^{2} \sin ^{2} \theta_{0} \\
P_{\text {output }} \cong \eta\left(M R^{\prime}\right)^{2} \frac{R\left(\theta_{0}\right) J^{2}}{8}\left(\frac{\theta_{\max }}{2 \sqrt{2}}\right)^{4} \cos ^{2} \theta_{0}
\end{gathered}
$$




$$
\begin{gathered}
M R^{\prime}=\frac{R_{\pi}-R_{0}}{R\left(\theta_{0}\right)} \\
\eta=\frac{4 R\left(\theta_{0}\right) Z_{0}}{\left(R\left(\theta_{0}\right)+Z_{0}\right)^{2}}
\end{gathered}
$$

where $P_{\text {Output }}$ and $P_{\text {Output }}^{\prime}$ are output power for the fundamental frequency and first harmonic frequency, respectively. $J$ is the current density required to excite the oscillation. $\theta_{0}$ and $\theta_{\max }$ are the average and maximum angle between the free layer and fixed layer magnetization during oscillation. $R(\theta)$ is the resistance when the relative angle between the two layer's magnetization is equal to $\theta . \eta$ corresponds to the impedance match between wave guide and the nano-pillar device. $\eta=1$ means a perfect impedance match (when $R\left(\theta_{0}\right)=Z_{0}$ ). In Eqs. (2-28) and (2-29), $\theta_{\max }$ is assumed to be small [4]. If $\theta_{\max }$ is large, $\frac{\theta_{\max }}{2}$ in (2-28) and $\left(\frac{\Delta \theta}{2 \sqrt{2}}\right)^{2}$ in (2-29) should be replaced by $B_{1}\left(\theta_{\max }\right)$ and $B_{2}\left(\theta_{\max }\right)$ respectively. Here, $B_{n}(\theta)$ is the Bessel function.

According to Eqs. (2-28) and (2-29), in order to achieve higher output power it is important to a have large MR ratio and to fabricate the device with an impedance match to the wave guide $(50 \Omega)$. It was reported that by replacing a GMR pillar with an MTJ the output power could be significantly increased [58, 61].

It has also been suggested that the output power could be increased by mutually phase-locking two nano-contact STNOs in close proximity [57, 59]. By changing the current passing through one of the STNOs, the oscillation frequencies of these two oscillators approached each other. When the difference of the two frequencies became 
small enough, the frequencies were suddenly synchronized and the two oscillators started to oscillate coherently at the same frequency. In addition, this coupling doubled the total output power of these two oscillators (the output was the square of the number of oscillators synchronized). In this way, if we can achieve coherent coupling of n oscillators, we may get $\mathrm{n}^{2}$ time larger output power [62].

\subsection{Magnetic anisotropy in thin films}

The advance of spintronics also relies on optimizing the ferromagnetic material's intrinsic properties. Magnetic anisotropy is one of the key properties that can be tuned. Ferromagnetic materials generally exhibit "easy" and "hard" directions that the magnetizations align along leading to different hysteresis loops in the two directions. The phenomenon is called magnetic anisotropy, which means the magnetic properties depend on the direction in which they are measured. The magnetic anisotropy is of considerable practical interest for various technological applications [63, 64]. For instance, materials with very large magnetic anisotropy are suitable in permanent magnets, while materials with small magnetic anisotropy are appropriate in magnetic field sensors.

\subsubsection{Magnetocrystalline anisotropy}

Magnetocrystalline anisotropy is intrinsic to the material and is due mainly to spin-orbit coupling [65]. The spins are coupled to the orbits through the spin-orbit 
interaction, while the orbit is strongly coupled to the lattice; as a result, the spins are affected by the crystal lattice. This results in the total magnetic energy being dependent on the orientation of the magnetization relative to the crystalline axis and symmetry of the crystal.
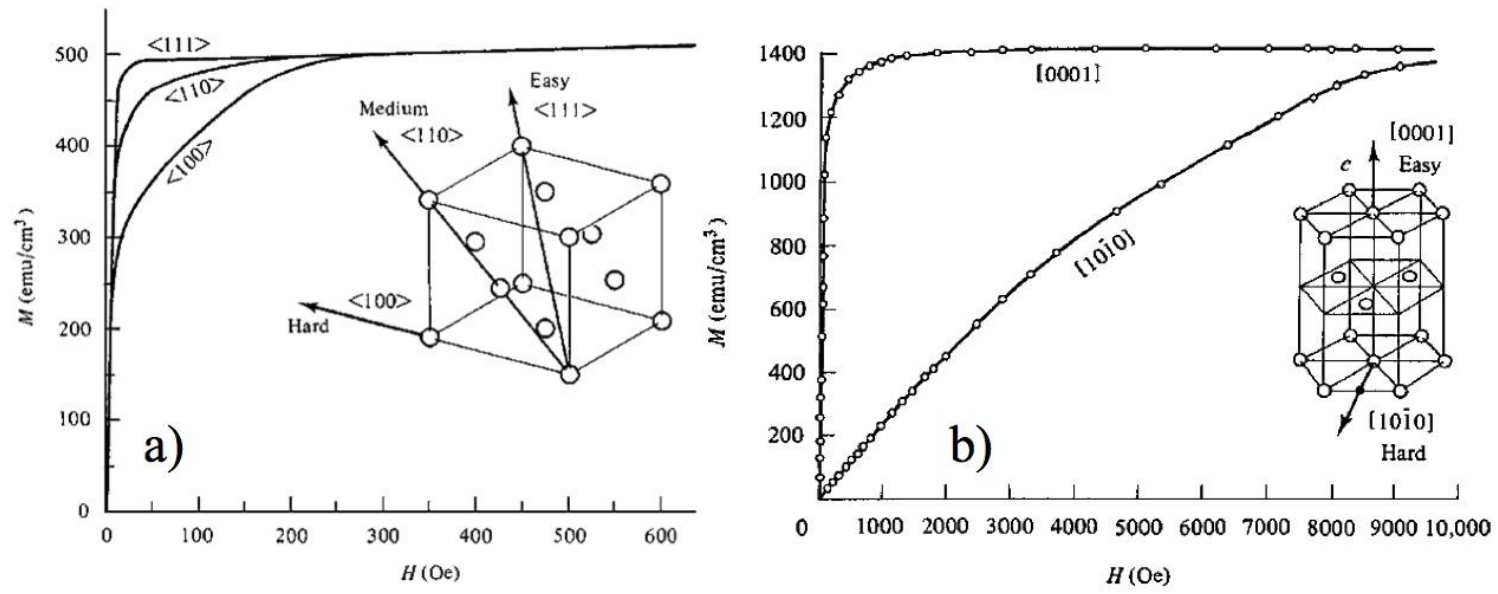

Figure 2.13 (a) Magnetization curves for single crystals of nickel with cubic structure. (b) Magnetization curves for singe crystal of cobalt with hexagonal close-packed structure. (Adapted from Ref. [65])

The magnetocrystalline anisotropy appears differently depending upon the symmetry of the material. The strength of the anisotropy is expressed by the magnitude of the anisotropy constants $K_{1}, K_{2}$, etc. In cubic crystals of high symmetry [for example, $\mathrm{Ni}$ as shown in Figure 2.13(a)], the anisotropy energy per volume can be written as [65]:

$$
E=K_{0}+K_{1}\left(\alpha_{1}^{2} \cdot \alpha_{2}^{2}+\alpha_{2}^{2} \cdot \alpha_{3}^{2}+\alpha_{3}^{2} \cdot \alpha_{1}^{2}\right)+K_{2}\left(\alpha_{1}^{2} \cdot \alpha_{2}^{2} \cdot \alpha_{3}^{2}\right)+\cdots
$$

where $\alpha_{1}, \alpha_{2}$, and $\alpha_{3}$ are the cosines of the angles that the magnetization $M s$ makes with 
respect to the crystal axes. $K_{1}, K_{2}$, etc. are expressed in $\mathrm{erg} / \mathrm{cm}^{3}$ (cgs) or $\mathrm{J} / \mathrm{m}^{3}$ (SI).

When $K_{2}$ is zero, the sign of $K_{1}$ determines the direction of easy axis. For positive $K_{l}$, $E_{100}<E_{110}<E_{111}$, and $<100>$ is the easy direction, for example, the single crystal iron. For negative $K_{1}, \quad E_{111}<E_{110}<E_{100}$, and $<111>$ is the easy axis. $K_{1}$ is negative for nickel.

When $K_{2}$ is not zero, the easy axis depends on the values of both $K_{1}$ and $K_{2}$. Table 2-1 summarizes the easy, medium, and hard direction in a cubic crystal for various ranges of constants.

Table 2-1 Directions of Easy, Medium, and Hard axis in a Cubic Crystal[65]

\begin{tabular}{|c|c|c|c|c|c|c|}
\hline$K_{1}$ & + & + & + & - & - & - \\
\hline$K_{2}$ & $+\infty$ to & $-9 K_{l} / 4$ to & $-9 K_{l} / 4$ to & $-\infty$ to & $9\left|K_{l}\right| / 4$ to \\
$9\left|K_{l}\right|$ & $9\left|K_{l}\right|$ to \\
$+\infty$
\end{tabular}

For structure of lower symmetry, such as a hexagonal closed-packed structure[Figure 2.13(b)], the anisotropy energy per volume can be described as [66]:

$$
E=K_{0}+K_{1} \sin ^{2} \theta+K_{2} \sin ^{4} \theta+\cdots
$$

where $\theta$ is the angle between $M s$ and the $c$ axis. With $K_{1}, K_{2}$ both positive, the energy $E$ is 
minimum for $\theta=0$, and the $c$ axis is the easy axis. A crystal with a single easy axis is referred as a uniaxial crystal. If $K_{1}, K_{2}$ are both negative, the minimum energy is obtained when $\theta=90^{\circ}$. This creates an easy plane of magnetization that is perpendicular to the $c$-axis in the hexagonal structure.

\subsubsection{Shape anisotropy}

A magnetized body will produce "magnetic charges" or poles at the surface. This surface "charge" distribution is itself another source of a magnetic field, called the demagnetizing field [67]. Magnetic shape anisotropy originates from the magnetic dipolar interaction, which is normally represented by the demagnetizing field. Since the internal demagnetizing field is less along a long axis than that along a short axis, it is naturally easier to induce a magnetization along the long direction of a non-spherical geometry than along the short direction. This is referred to as the shape anisotropy. In the thin film samples, the shape anisotropy is particularly important because the thickness of the thin film is normally much shorter than the in-plane length or width. Thus, the shape anisotropy is primarily responsible for the in-plane magnetization usually observed.

The demagnetizing field $H_{d}$ is proportional to the magnetization that creates it [68]:

$$
\vec{H}_{d}=-N_{d} \vec{M}
$$

where $N_{d}$ is the demagnetizing factor and depends mainly on the shape of the magnet. The sum of the demagnetizing factor is a constant: 


$$
N_{a}+N_{b}+N_{c}=4 \pi
$$

where $a, b$ and $c$ describe the 3 dimensions of a magnet. For continuous thin films that have much smaller thickness than the length and width, thus all factors are zero except for the direction perpendicular to the layer ( $N a$ for instance). In order to treat shape anisotropy quantitatively, the magnetostatic energy $E_{m s}$ is expressed as:

$$
E_{m s}=-\frac{1}{2} \vec{H}_{d} \cdot \vec{M}=\frac{1}{8 \pi} \int H_{d}^{2} d v
$$

where $d v$ is an element of volume and the integration extends over all space.

For thin films, the magnetostatic energy becomes:

$$
E_{m s}=\frac{1}{2}\left(M_{S} \cos \theta\right)^{2} N_{a}=2 \pi M_{S}^{2} \cos ^{2} \theta
$$

where $\theta$ is the angle between $M$ and the direction perpendicular to the film. The shape anisotropy is given by:

$$
K_{S}=E_{m s}(\theta=0)-E_{m s}\left(\theta=\frac{\pi}{2}\right)=2 \pi M_{S}^{2}
$$

Eq. (2-38) indicates that in the thin film geometry the magnetostatic energy favors an in-plane orientation for the magnetization with an anisotropy energy barrier of $2 \pi M_{S}^{2}$.

\subsubsection{Perpendicular magnetic anisotropy (PMA)}

It has been experimentally revealed that many magnetic thin films or multilayers exhibit strong perpendicular magnetic anisotropy (PMA) that can overcome the in-plane shape anisotropy. The perpendicular magnetic anisotropy energy constant $K_{P}$ can be 
phenomenologically divided into two contributions as [69, 70]:

$$
K_{P}=K_{e f f}=K_{V}+\frac{K_{S}}{t}
$$

Where $K_{\text {eff }}$ is the effective anisotropy energy per volume, $K_{V}$ is the bulk contribution of the anisotropy energy, $K_{S}$ is the interfacial contribution of the anisotropy energy, and $t$ is the thickness of the magnetic layer. In this work, I will introduce the strong PMA material of $\mathrm{L} 1_{0} \mathrm{MnAl}$ with $K_{V}$ being the dominant contribution from magnetocrystalline anisotropy. The $K_{S}$ can be attributed to an Néel-type surface contribution [71]. It has been suggested that $K_{S}$ was the main contribution leading to the PMA at a $\mathrm{Co}_{2} \mathrm{Fe} \mathrm{Al} / \mathrm{MgO}$ interface [72]. $K_{\text {eff }}$ can be expressed in terms of the uniaxial anisotropy energy and the in-plane shape anisotropy:

$$
K_{\text {eff }}=\left|K_{U}\right|-2 \pi M_{S}^{2}
$$

where $K_{U}$ is the uniaxial anisotropy arising from the magnetocrystalline anisotropy or interface anisotropy. Keff must be greater than zero for overall perpendicular remanence. Thus $K_{U}$ can be obtained from Eq. (2-40) [73].

The perpendicular magnetic anisotropy $\left(K_{e f f}\right)$ can be measured by fitting the hysteresis loop or by calculating, on a graph of $M-H$ loop, the area included between the magnetization curves between the perpendicular and in-plane directions, the demonstration of this for $\mathrm{L} 1_{0} \mathrm{MnAl}$ can be found in Chapter 5 and Ref.[65]. The anisotropy energy equals the energy stored in a crystal when it is magnetized to saturation in a non-easy direction. Hence, if we can determine the work done on the crystal to bring it to saturation, we can 
equate $E$ and $W$ and so determine the anisotropy constants. The work done in magnetization is simply the area between the $M-H$ curve and the $M$-axis and is evaluated as:

$$
E=W=\int_{0}^{M} H d M
$$

where $M$ is in emu/ $\mathrm{cm}^{3}$, and $E$ is in $\mathrm{erg} / \mathrm{cm}^{3}$. Note the area method yields anisotropy constants assuming no domain wall pinning effect [74].

\subsection{Material candidates for STNO}

\subsubsection{Heusler alloy $\mathrm{Co}_{2} \mathrm{FeAl}$}

The Heusler alloy $\mathrm{Co}_{2} \mathrm{FeAl}$ has attracted a great deal of interest in application to STT-MRAM due to the prediction of high spin polarization and low damping parameter [75]. It was considered as a promising electrode for perpendicular MTJs because of the PMA at the $\mathrm{Co}_{2} \mathrm{FeAl} / \mathrm{MgO}$ interfaces [72].

Although the spin polarization efficiency was predicted to be $\sim 100 \%$ in ideal Heusler-alloys that exhibit a band gap in the minority spin, $\mathrm{Co}_{2} \mathrm{Fe} \mathrm{Al}$ is not considered as an ideal half metal $[76,77]$. Miura etc. suggested that the charge transfer from the Co minority spin states to the Fe minority spin states results in the Fe 3d minority conduction band shifting towards the Fermi level and brings in additional states, thus destroying the half 
metallicity [77]. The theoretical calculation yielded a spin polarization in $\mathrm{Co}_{2} \mathrm{FeAl}$ about $30 \%$ using the KKR method[77] and 38\% by the SPD method [78].

\subsubsection{L1 $1_{0} \mathrm{MnAl}$}

Permanent magnetic thin films with perpendicular magnetic anisotropy (PMA) have been of great interest due to their application in perpendicular magnetic recording and spintronic devices $[79,80]$. Among the $\mathrm{L}_{0}$ intermetallics, MnAl shows attractive magnetic properties [81, 82]. Bulk MnAl has been reported with the saturation magnetization of 490 $\mathrm{emu} / \mathrm{cm}^{3}$ along with large perpendicular uniaxial magnetocrystalline anisotropy $K_{U} \sim 10^{7}$ $\mathrm{erg} / \mathrm{cm}^{3}$ [83]. The high $K_{U}$ makes it promising as a magnetic fixed layer for perpendicular magnetic tunnel junctions (p-MTJs) [84]. Moreover, the low damping constant of MnAl $(\sim 0.006)[84]$ is very desirable for low energy dissipation spin torque devices such as spin-transfer torque magnetic tunnel junctions and spin torque nano-oscillators (STNO) [85]. Typically STNO operation requires very large external magnetic fields, however recent innovation utilizing PMA materials has made it possible to operate at low field or even at zero field [13-17].

The effect of magnetic domain walls (DWs) on the spin transfer torque behavior of nano-structures, e.g. nano-wire particularly, is a topic of great interest for fundamental studies and for domain wall motion related applications [86-88]. However, the 
measurement of DWs' resistance remains challenging because the resistance from DWs scattering is very small and can be comparable with the anisotropic magneto-resistance (AMR). Epitaxial films with strong perpendicular magnetic anisotropy, such as hcp(0001) Co [89, 90], L1 ${ }_{0} \mathrm{FePt}[91]$ and FePd [92], are ideal systems for studying DWs resistance because the magnetization inside the domains is always perpendicular to the current, which excludes the AMR effect.

The metastable $\tau$ phase of $\mathrm{MnAl}$ is the only ferromagnetic phase of this binary intermetallic and only forms in a relatively small window of chemical composition close to $\mathrm{Mn}_{50} \mathrm{Al}_{50}$ [83]. Ferromagnetic $\tau-\mathrm{MnAl}$ has an $\mathrm{L}_{0}$ structure, which can be reduced to a tetragonally distorted B2 ordered structure with a lattice parameter c $\sim 3.57 \AA$ (Figure 2.14) [83, 93]. The chemical ordering between $\mathrm{Mn}$ and $\mathrm{Al}$ is very important for the ferromagnetism in $\tau$-MnAl. According to the Bethe-Slater curve, a positive exchange interaction between 3d-electrons of adjacent $\mathrm{Mn}$ atoms tending to align the Mn moments ferromagnetically when the neighboring Mn has an interatomic distance larger than $2.57 \AA$ [94]. Epitaxial growth of the film is therefore necessary to achieve a high chemical ordering. 


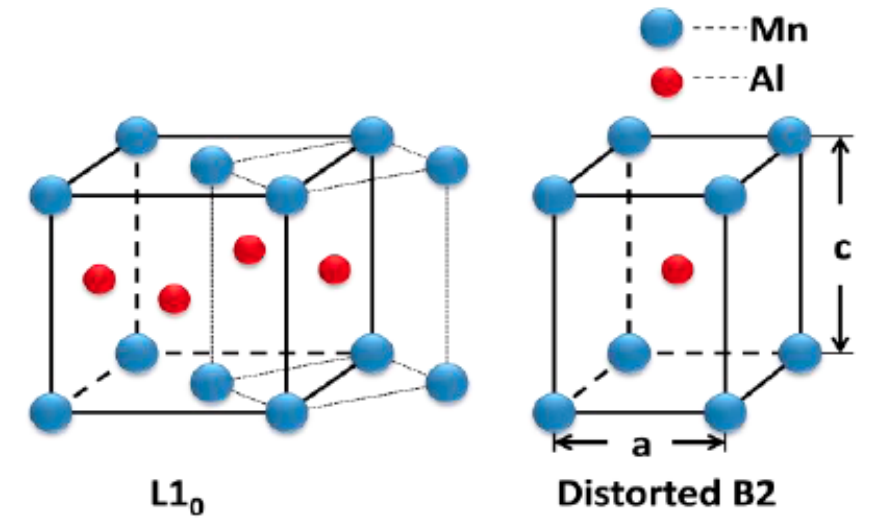

Figure 2.14 $\mathrm{L}_{0}$ structure of $\tau-\mathrm{MnAl}$, which can be reduced into a distorted $\mathrm{B} 2$ structure. (Adapted from Ref.[95]) 


\section{Chapter 3 Experimental techniques}

\subsection{Multilayer film fabrication by RBTIBD}

Up to date, magnetron (or RF diode) sputtering and ion beam deposition are the two deposition methods most successfully applied to deposit magnetic multilayers. However, theses two conventional methods were not ideally suited for optimal GMR structure deposition. For example, magnetron and RF diode systems operate at high pressures and the adatom flux is substantially scattered by the low energy background gas during transport from target to substrate [96]. The ion-beam deposition, on the other hand, can only be operated using relatively high sputtering ion energies and relatively low pressure with no substantial background gas scattering [97]. This is because at low beam voltage, it is more difficult to focus the sputtering ion beam, meaning a larger fraction of the ion beam could miss the targets and sputter off undesired materials from the vacuum system hardware, resulting in overspill contamination [98]. Using higher acceleration voltages can better focus the ion beam on the target. Unfortunately, higher accelerating voltage gives the adatoms higher kinetic energies when they reach the substrate, causing intermixing at the interfaces, which can significantly damage the GMR properties [99]. Low energy IBD would improve the uniformity of the film thickness and reduce interlayer mixing but requires a large target to avoid overspill sputtering. Because the majority of the ions are focused only near the center of the target, this results in poor utilization of the target 
material.

The reactive biased target ion beam deposition (RBTIBD) technique was developed to overcome the problems of the conventional magnetron sputtering and IBD method [100, 101]. RBTIBD allows a low energy ion beam deposition while keeping the overspill contamination minimized [101]. One of the drawbacks of RBTIBD is that it cannot directly sputter oxide targets. This limitation can be overcome by introducing oxygen during the sputtering processes. The RBTIBD system is co-designed by University of Virginia and 4-Wave Inc. The magnetic multilayer stacks discussed in this work were all deposited in the RBTIBD system.

RBTIBD has a main process chamber connected to a cryogenic pump, maintaining its base pressure $\sim 10^{-8}$ Torr. A load lock houses a substrate stage assembly, allowing sample loading and retrieving without breaking the main chamber vacuum. A mechanical pump is used to pump down the load lock down to $\sim 75 \mathrm{mT}$ before opening the isolation gate valve between the main chamber and load lock chamber. Cooling water is provided to both the system and the compressor of the cryogenic pump by a chiller.

Ultra high purity (UHP) Ar gas (99.999\%) is used by the ion source to generate the ion beam plasma. There are three gas lines connected to the ion source in the chamber. Two of them are Ar gas lines, one through the anode and the other through the cathode. An additional line of $\mathrm{O}_{2}$ gas or $\mathrm{O}_{2} / \mathrm{Ar}$ mixture can be activated during sputtering when necessary for oxide deposition. The gas flow rates are controlled by digital mass flow 
controllers $\left(\mathrm{MFC}, \mathrm{MKS}^{\mathrm{TM}}\right)$.

The key component of the novel RBTIBD system is the low energy broad beam ion source, and it is the combination of a Mark $\mathrm{II}^{\mathrm{TM}}$ end-Hall ion source and and HCES 5000 hollow cathode electron source as shown in Figure 3.1. The Mark II end-Hall ion source acts as the anode ionizing the inert Ar gas fed into the source. The end-Hall ion source is made with hard and soft magnetic materials and a gas inlet. The hollow cathode consists of a narrow tube, a "keeper" surrounding the cathode, and an emission end. The hollow cathode generates the electrons that flow toward to the anode of the end-Hall source where the ions are created. The electric and magnetic fields near the anode determine both ion and electron path lengths, control the ionization in the discharge, as well as accelerate the ions out of the anode [101]. The most essential advantage of this ion source is that it can provide inert ions with very low energy (5 eV-50 eV) and rather broader energy range. This feature is in sharp contrast to the conventional IBD system equipped with grid ion beam source where only ions narrowly distributed in a high energy range are allowed so that the ion beam can be focused onto the target to reduce overspill contamination. 


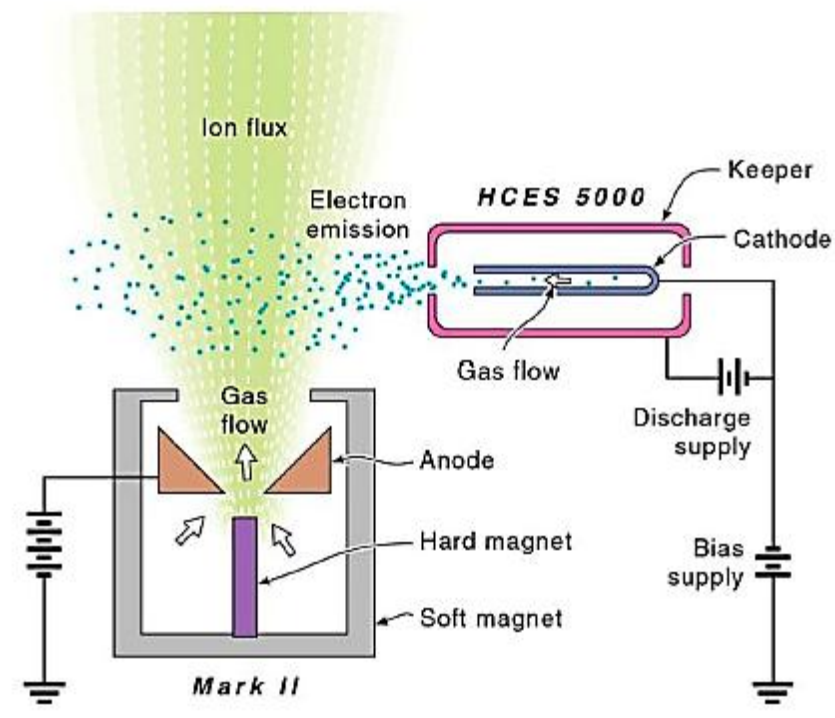

Figure 3.1 Schematic layout of Mark II End-Hall ion source and HCES 5000 Hollow Cathode electron source. (Reproduced from Ref.[95])

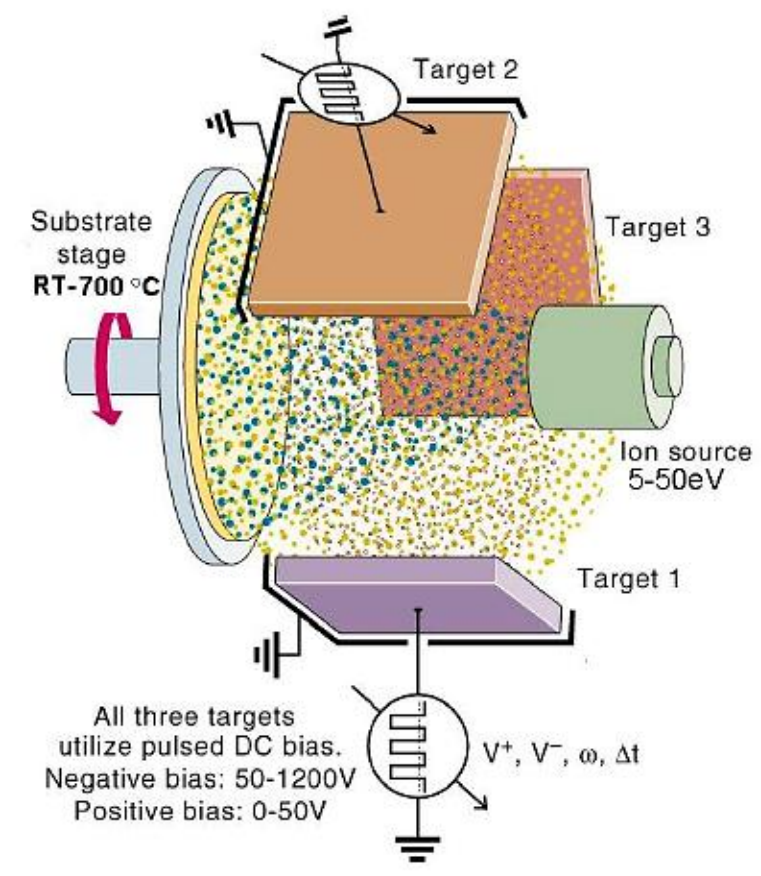

Figure 3.2 The schematic of the RBTIBD processing chamber. (Reproduce from Ref. [95]) 
The sputtering of biased targets happens in the processing chamber. The schematic illustration of the RBTIBD processing chamber is shown in Figure 3.2. Six $100 \mathrm{~mm}$ diameter metallic targets sit in a hexagonal housing facing each other. The ion source and rotatable $100 \mathrm{~mm}$ diameter sample stage lie along the center axis on either side of the target housing. A two-stage target shutter carousel (not shown in Figure 3.2) simultaneously exposes three targets, while blocking the other three targets.

A large negative bias (up to $1.2 \mathrm{kV}$ ) and a small positive one (up to $50 \mathrm{~V}$ ) are applied alternatively to the target (pulsed DC bias mode). During the negative bias the ions are accelerated toward the target, inducing sputtering. During a positive bias, no sputtering occurs, but the target poisoning in oxide depositions can be reduced effectively by discharging any dielectric material buildup during the negative bias. The pulse width and frequency of the DC bias can be programmed independently for the three channels. As shown in Figure 3.3, two targets are connected to a pulsar through a relay switch. The two-stage target shutter exposes three targets simultaneously $(1 / 3 / 5$ or $2 / 4 / 6)$ to the ion beam but only the biased targets are sputtered. The design of the target shutter allows co-sputtering of up to three targets. By systematic modulation of the relative pulse period of each selected target during the co-sputtering process, a wide range of phase space in alloy material system can be explored.

It is worth mentioning that part of the ion beam will directly bombard the samples on the substrate stage with an un-accelerated low energy $(\sim 40 \mathrm{eV})$ beam, resulting in 
un-desired surface roughness. To minimize this effect, a beam blocker is installed between the anode and the substrate stage, which significantly reduces the direct ion bombardment.

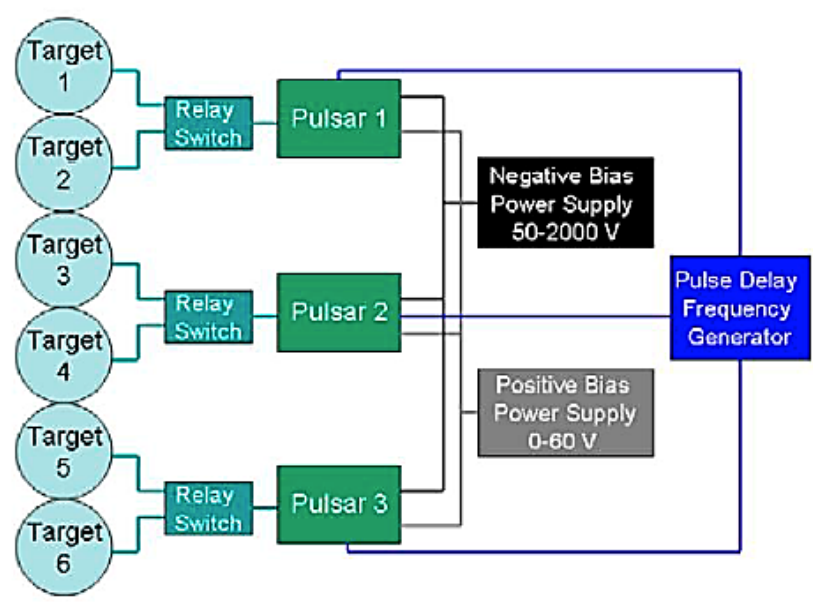

Figure 3.3 Schematics of the pulsed DC bias control. (Reproduced from Ref.[29])

The RBTIBD system also has an advantage of making depositions at elevated temperatures by using a heated sample stage. As shown in Figure 3.2, the sample stage is vertically positioned and rotates along the same axis shared by the ion source during the deposition. Tantalum was chosen as the material for the wafer carrier because of its relatively high melting temperature, and low thermal expansion coefficient. An infrared heating lamp (Heraeus Nobel Light 80mm Omega Emitter) installed right behind the wafer carrier helps to heat the stage. The stage temperature can be controlled by a variable voltage input according to a pre-determined calibration curve. The target surface temperature calibration is carried out by attaching a standard K-type thermocouple directly 
to the sample stage surface and reading out the temperature value via a vacuum feed-through. The infrared lamp is capable of heating the stage up to $550^{\circ} \mathrm{C}$.

For the multilayer preparation, the base pressure is pumped down below $8 \times 10^{-8}$ Torr before sputtering. The substrates are exposed to the low energy $(\sim 35 \mathrm{eV})$ un-accelerated ions for 5 minutes to remove the surface contamination before the deposition.

Post-annealing treatments are used to improve the crystallinity of the thin films or to establish the exchange bias for MTJs. The furnace in our lab uses forming gas (95\% Ar and $5 \% \mathrm{H}_{2}$ ) to prevent surface oxidation during annealing. The magnetic field can be adjusted up to $3 \mathrm{kOe}$ using an electromagnet. The set point and ramp-up rate for the temperature can be controlled by a PID (proportional-integral-derivative feedback controller) system. Rapid thermal annealing (RTA) in the UVa clean room facility is also used for some of my multilayers allowing for a much faster heating and cooling process. The RTA process was

controlled by a thermocouple in a vacuum environment of $\sim 10^{-5}$ Torr. The annealing temperature can be adjusted from $350{ }^{\circ} \mathrm{C}$ up to $600{ }^{\circ} \mathrm{C}$ with a ramp rate up to $20^{\circ} \mathrm{C} / \mathrm{s}$.

\subsubsection{Deposition of epitaxy $\mathrm{MnAl}$ on $\mathrm{MgO}(001)$ substrate}

A MnAl film was synthesized by co-sputtering of $\mathrm{Mn}$ and $\mathrm{Al}$ targets in the Biased Target Ion Beam Deposition system (BTIBD). Cubic $\mathrm{MgO}(001)$ substrates were selected 
to provide a seeding effect. The complete structure of the thin film was $\mathrm{MgO}$ (substrate) / $40 \mathrm{~nm} \mathrm{Cr} / 20 \mathrm{~nm} \mathrm{MnAl} / 10 \mathrm{~nm}$ Cr. In order to further reduce the lattice mismatch between the $\mathrm{MgO}$ substrate and the $\mathrm{MnAl}(\sim 6.7 \%)$, a $40 \mathrm{~nm}$ Cr buffer layer is used. Cr has a Body Centered Cubic (BCC) structure with a lattice constant of $\sim 2.89 \AA$ when grown on $\mathrm{MgO}(001)$ substrates. The thin $\mathrm{Cr}$ capping layer was added to prevent $\mathrm{MnAl}$ from oxidization.

During the co-sputtering process, the relative compositions of $\mathrm{Mn}$ and $\mathrm{Al}$ were well controlled to be $\sim 50: 50$ by varying the target pulse frequency and positive pulse width. The base pressure of the vacuum chamber is $\sim 5 \times 10^{-8}$ Torr. The deposition pressure is $\sim 1.60 \times 10^{-4}$ Torr with the flowing of Ar gas. Prior to the deposition, the MgO substrates were in situ annealed at $500^{\circ} \mathrm{C}$ for 10 minutes, the surface of which was cleaned by Ar ions with an energy of $\sim 35 \mathrm{eV}$ for 5 minutes. Prior to the growth of each layer, the corresponding targets were pre-sputtered for 5 minutes to remove any possible contamination during the deposition. The growth temperature was set to $200^{\circ} \mathrm{C}$ for a preliminary crystallization of $\mathrm{Cr}$ and $\mathrm{MnAl}$ layers and to enhance the epitaxial growth of each layer. The samples were post-annealed at $400^{\circ} \mathrm{C}$ for 1 minute in a Rapid Thermal Annealing (RTA) furnace under vacuum condition.

For characterization, with the techniques introduced in the following sections, the film thickness and phase composition were characterized using a high resolution X-ray diffractometer (Smart-lab, Rigaku Inc.). The in-plane and out-of-plane magnetic hysteresis 
loops were measured by a vibrating sample magnetometer from $50 \mathrm{~K}$ to $320 \mathrm{~K}$ (PPMS 6000, Quantum Design). Lastly, a CoCr coated Si tip was used in a magnetic force microscope (Cypher, Asylum Research) to study the magnetic domain structure at room temperature.

\subsection{Characterization}

\subsubsection{X-ray diffraction (XRD)}

The crystal structures of the thin films are characterized using X-ray diffraction techniques. According to Bragg's law, $2 d \sin \theta=n \lambda$ where $\mathrm{n}$ is an integral, and $\lambda$ is the wavelength of the incident wave. One can determine the crystal structure and lattice parameters $(d)$ according to the diffraction angles $(\theta)$ of the Bragg peaks, or predict the positions of the Bragg peaks with knowledge of the crystal structure. Besides, one can determine the degree of certain chemical ordering based on the diffraction patterns, that is important for ordered alloys such as $\mathrm{L} 1_{0} \mathrm{MnAl}$.

A Rigaku Smart lab XRD system performed all the structure characterizations for this work especially for $\mathrm{L} 1_{0} \mathrm{MnAl}$, the XRD result for which will be discussed in detailed in Chapter 5. XRR is another option in XRD to characterize the thickness of the multilayer films and is used for thickness characterization of all the multilayer structures in this work.

The X-ray source is operated at an accelerating voltage of $44 \mathrm{keV}$ and $40 \mathrm{Amps}$. $\mathrm{Cu} \mathrm{Ka}$ 
radiation is used with a wavelength $\sim 1.54 \AA$. $\mathrm{A} G(2 \times 220)$ mirror monochromator was used for high resolution X-ray experiments.

\subsubsection{Physics property measurement system (PPMS)}

Most of the characterization of the magnetic properties that is discussed later in the dissertation including the hysteresis loops and the magneto-transport is carried out by the PPMS system. There are two options in the PPMS system, the Vibrating Sample Magnetometer (VSM) option and the Electrical Transport Option (ETO). The basic measurement of the VSM is accomplished by oscillating the sample near a detection (pickup) coil and synchronously detecting the voltage induced. The basic principle of operation for a vibrating sample magnetometer is that a changing magnetic flux will induce a voltage in a pickup coil. The time-dependent induced voltage is given by the following equation:

$$
V_{\text {Coil }}=\frac{d \Phi}{d t}=\left(\frac{d \Phi}{d z}\right)\left(\frac{d \mathrm{z}}{d t}\right)
$$

In Eq.(3-1), $\Phi$ is the magnetic flux enclosed by the pickup coil, $z$ is the vertical position of the sample with respect to the coil, and $t$ is time. For a sinusoidally oscillating sample position, the voltage is based on the following equation:

$$
\text { Vcoil }=2 \pi f C M A \sin (2 \pi f t)
$$

In Eq.(3-2), $C$ is a coupling constant, $M$ is the DC magnetic moment of the sample, $A$ is 
the amplitude of oscillation (1-3mm), and $f$ is the frequency of oscillation $(40 \mathrm{~Hz})$. By measuring the coefficient of the sinusoidal voltage response, the magnetic moment can be acquired. A lock-in circuit is utilized when measure the induced voltage to exclude the effect from the flux change of external fields.

The magnetic hysteresis loops are measured using the VSM option in PPMS 6000 (Quantum Design) and Versalab (Quantum Design). Both systems are equipped with a superconducting magnet providing a maximum field up to $70 \mathrm{kOe}$ for PPMS and $30 \mathrm{kOe}$ for Versalab. The temperature range can be adjusted from $2 \mathrm{~K} \sim 300 \mathrm{~K}$ in the PPMS and from $50 \mathrm{~K} \sim 400 \mathrm{~K}$ in the Versalab system. The system is especially useful to study the temperature dependent magnetic behaviors.

The ETO mode has a 2-channel measurement system that uses an AC current excitation $(0.4-177 \mathrm{~Hz})$ and lock-in voltage detection to perform 4-wire measurements of electrical resistance, $I-V$ curve, or Hall effect in samples with resistances of up to several mega-ohms. It is worth mentioning that the current source can superimpose a DC bias current $I_{\text {bias }}(10 \mathrm{nA}-70 \mathrm{~mA})$ on an AC signal, making it possible to use the lock-in to study differential resistance $d V / d I$ versus $I_{\text {bias }}$.

One big advantage for the ETO mode is that it can carry out the electrical measurement with adjustable external magnetic field under different temperatures. This enables us to study the magneto-transport properties, such as GMR/TMR, of the magnetic structures with at different magnetic fields and at different temperatures. The differential resistance 
function is applied for the spin-transfer study in nano-sized devices and will be discussed in Chapter 7.

\subsubsection{Scanning electron microscope (SEM)}

The SEM (ZEISS Gemini) in the UVa clean room facility is used during the nano-device fabrication discussed in this work (Chapter 6). The scanning electron microscope has advantages over a conventional light microscope in its high magnification and large depth of field. With the help of the SEM, the three dimensional (3D) appearance of the patterned sample can be obtained.

For routine scanning electron microscope images, secondary electrons (SE) form the usual image of the surface [102]. Secondary electrons are low energy electrons formed by inelastic scattering and have energy of less than $50 \mathrm{eV}$. The low energy of these electrons allows them to be collected easily. This is achieved by placing a positively biased grid on the front of the SE detector, which is positioned off to one side of the specimen. The positive grill attracts the negative electrons and they go through it into the Everhart-Thornley detector.

The major influence on SE signal-generation is the shape (topography) of the specimen surface [103]. Secondary electrons provide particularly good edge detail. Edges (and often pointy parts) look brighter than the rest of the image because they produce more 
electrons. A tilting angle of 45 degree is normally applied to the sample stage during the imaging of the patterned sample to better inspect the edge or sidewall of the devices.

\subsubsection{Atomic force microscope (AFM)}

AFM was heavily used to inspect the morphology and surface roughness for films deposited by the RBTIBD system. Besides, it helps to visualize the 3-D image of the nano-devices and can provide height profile information. AFMs operate by measuring the force between a probe and the sample. The probe typically has a sharp tip, which is a 3 6 $\mu \mathrm{m}$ tall pyramid with $15 \sim 40 \mathrm{~nm}$ end radius (Figure 3.4 shows the SEM images for Si AFM tip). Though the lateral resolution of AFM is low $(\sim 30 \mathrm{~nm})$ due to the convolution, the vertical resolution can be up to $0.1 \mathrm{~nm}$.

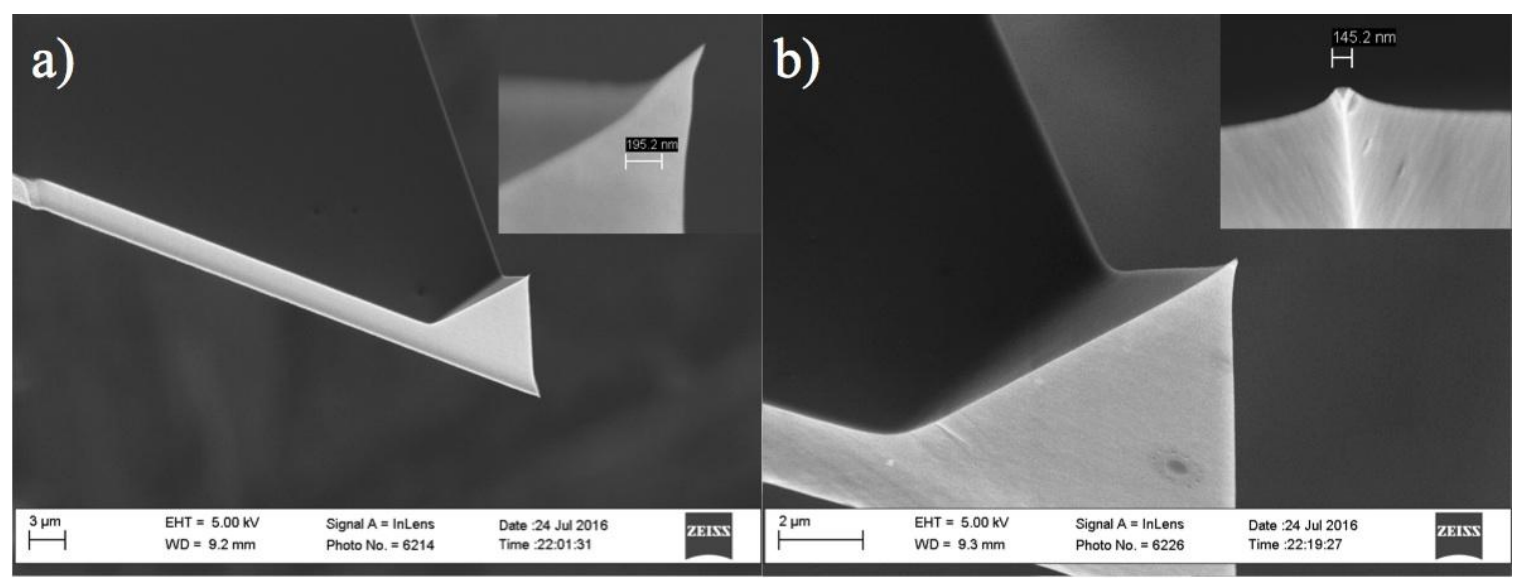

Figure 3.4 (a) SEM image for a new AFM tip; the inset shows the very end of the tip. (b)

SEM image of a worn-out tip. The inset shows the end of tip with a flattened triangle shape. 
To acquire the image resolution, AFMs can generally measure the vertical and lateral deflections of the cantilever by using an optical lever. The optical lever operates by reflecting a laser beam off the cantilever. The reflected laser beam strikes a position-sensitive photo-detector consisting of four-segment photodiode arrays. The differences between the segments of photo-detector of signals indicate the position of the laser spot on the detector and thus the angular deflections of the cantilever.

The scanner is made of peizo-ceremics. Piezoelectric ceramics are a class of materials that expand or contract when in the presence of a voltage gradient. Piezo-ceramics make it possible to create three-dimensional positioning with high precision. During the scan, there is a "fast" axis and "slow" axis. The combining of both scan result in a zig-zag scanning trajectory across the sample surface.

In contact mode, AFMs use feedback to regulate the force on the sample. The AFM not only measures the force on the sample but also regulates it, allowing acquisition of images at very low forces. The feedback loop consists of the tube scanner that controls the height of the tip; the cantilever and optical lever, which measures the local height of the sample; and a feedback circuit that attempts to keep the cantilever deflection constant by adjusting the voltage applied to the scanner.

In a tapping mode, a cantilever is oscillating in free air at its resonant frequency. A piezo excites the cantilever's substrate vertically, causing the tip to bounce up and down. 
As the cantilever bounces vertically, the reflected laser beam is deflected in a regular pattern over a photodiode array, generating a sinusoidal electronic signal. Although the piezo continues to excite the cantilever's substrate with the same energy, the tip is deflected when encountering the surface. The reflected laser beam reveals information about the vertical height of the sample surface and some characteristics of the sample material itself. These material characteristics may include elasticity ("hardness"), magnetic and/or electric forces [104].

\subsubsection{Magnetic force microscope (MFM)}

MFM was used to characterize the magnetic domain information and MFM images were discussed in detail in Chapter 5. In MFM, a tapping cantilever equipped with a special tip is first scanned over the surface of the sample to obtain topographic information. The tip is then raised just above the sample surface using lift-mode. The surface topography is scanned while being monitored for the influence of magnetic forces. These influences are measured using the principle of force gradient detection. In the absence of magnetic forces, the cantilever has a resonant frequency $f_{0}$. This frequency is shifted by an amount $\Delta f$ proportional to vertical gradients in the magnetic forces on the tip. The shifts in resonant frequency tend to be very small, typically in the range $1-50 \mathrm{~Hz}$ for cantilevers having a resonant frequency $f_{0} \sim 100 \mathrm{kHz}$ [104]. The frequency shifts is reflected on the phase image, 
on which the contrast indicates the different magnetization direction. MFM is used to image the magnetic domains for $\mathrm{MnAl}$ and will be referred in Chapter 5 .

\subsection{Micromagnetic simulation}

To gain a full understanding of the spin torque switching and oscillation, micromagnetic simulations were carried out based on the Landau-Lifshitz-Gilbert-Slonczewski (LLGS) equation [105, 106]:

$$
\frac{d \vec{M}}{d t}=\gamma \vec{M} \times \vec{H}_{e f f}-\frac{\alpha}{M_{S}}\left(\vec{M} \times \frac{d \vec{M}}{d t}\right)-\frac{\gamma \hbar}{2 \mu_{0} e} \frac{J g(\theta, P)}{M_{S} d} \vec{M} \times\left(\vec{M} \times \vec{m}_{f i x}\right)
$$

where $\vec{M}$ is the unit vector of the free layer magnetization, $M_{S}$ is the saturation magnetization of the free layer, $\vec{m}_{f i x}$ is the unit vector of the reference layer magnetization, $\vec{H}_{e f f}$ is the effective magnetic field, $\alpha$ is the Gilbert damping constant, $\gamma=g \mu_{B} / \hbar$ is the gyromagnetic ratio expressed through the Lande g-factor, Bohr magneton $\mu_{B}$, and Planck constant $\hbar, J$ is the current density, $d$ is the free layer thickness. $g(\theta, P)$ is the scalar factor depending on the spin polarization $P$ and the angle $\theta$ between the reference layer and free layer's magnetization. The first term is the precession torque in the effective field $\vec{H}_{e f f}$, the second term is the Gilbert damping torque, and the third term is the spin-transfer torque.

Micromagnetic simulations were carried out based on the LLG equation including a spin-torque term [37]. The parameters used to model the $\mathrm{NiFe}$ layer were $M_{S}=650 \mathrm{emu} / \mathrm{cm}^{3}$, 
exchange stiffness constant of $A=1.3 \times 10^{-6} \mathrm{erg} / \mathrm{cm}$, and damping constant of $\alpha=0.009$. The mesh size was $10 \times 10 \times 3.25 \mathrm{~nm}^{3}$. The spin polarization parameter $P$ was assumed to be 0.4 . 


\section{Chapter 4 Magnetic multilayers with $\mathrm{Co}_{2} \mathrm{FeAl}$}

\subsection{Introduction}

In this chapter, the effort to engineer the structure of $\mathrm{Co}_{2} \mathrm{FeAl}$ based magnetic multilayers for use as a spin-torque oscillator by varying the spacer material and adding a nano-oxide layer (NOL) is described. Magnetic static and magneto-transport properties of the different magnetic multilayer structures are discussed in details. To improve the magnetoresistance ratio (MR) of those multilayers, different approaches including adjusting of spacer thicknesses, changing of spacer materials and adding of NOL are implemented.

\subsection{CFA/Cr/CFA structure}

\subsubsection{Effect of Cr spacer thickness}

For a pseudo spin-valve (PSV), the separation of magnetic coercive field between the reference layer and free layer is very important since there is no pinning layer. One way to achieve the magnetic separation is to tune the spacer thickness to have an antiferromagnetic coupling. In order to look at the interlayer couplings, magnetic hysteresis loops for $\mathrm{CFA}(5 \mathrm{~nm}) / \mathrm{Cr}(\mathrm{x}) / \mathrm{CFA}(5 \mathrm{~nm}) / \mathrm{Al}(5 \mathrm{~nm})$ with different $\mathrm{Cr}$ spacer thicknesses were measured as shown in Figure 4.1. In the case of $\mathrm{Cr}=3 \mathrm{~nm}, 4 \mathrm{~nm}, 5 \mathrm{~nm}$ and 
$6 \mathrm{~nm}$, the two magnetic layers are ferromagneticlly coupled with each other leading to a single hysteresis loop. The M-H loops for them are similar to the CFA single layer M-H loop [Figure 4.1(a)] with a coercive field $H_{C} \sim 20$ Oe. For $\mathrm{Cr}=1.5 \mathrm{~nm}$, the interlayer coupling is antiferromagnetic, where the loop is split and each layers' moment can be determined[Figure 4.1(f)].
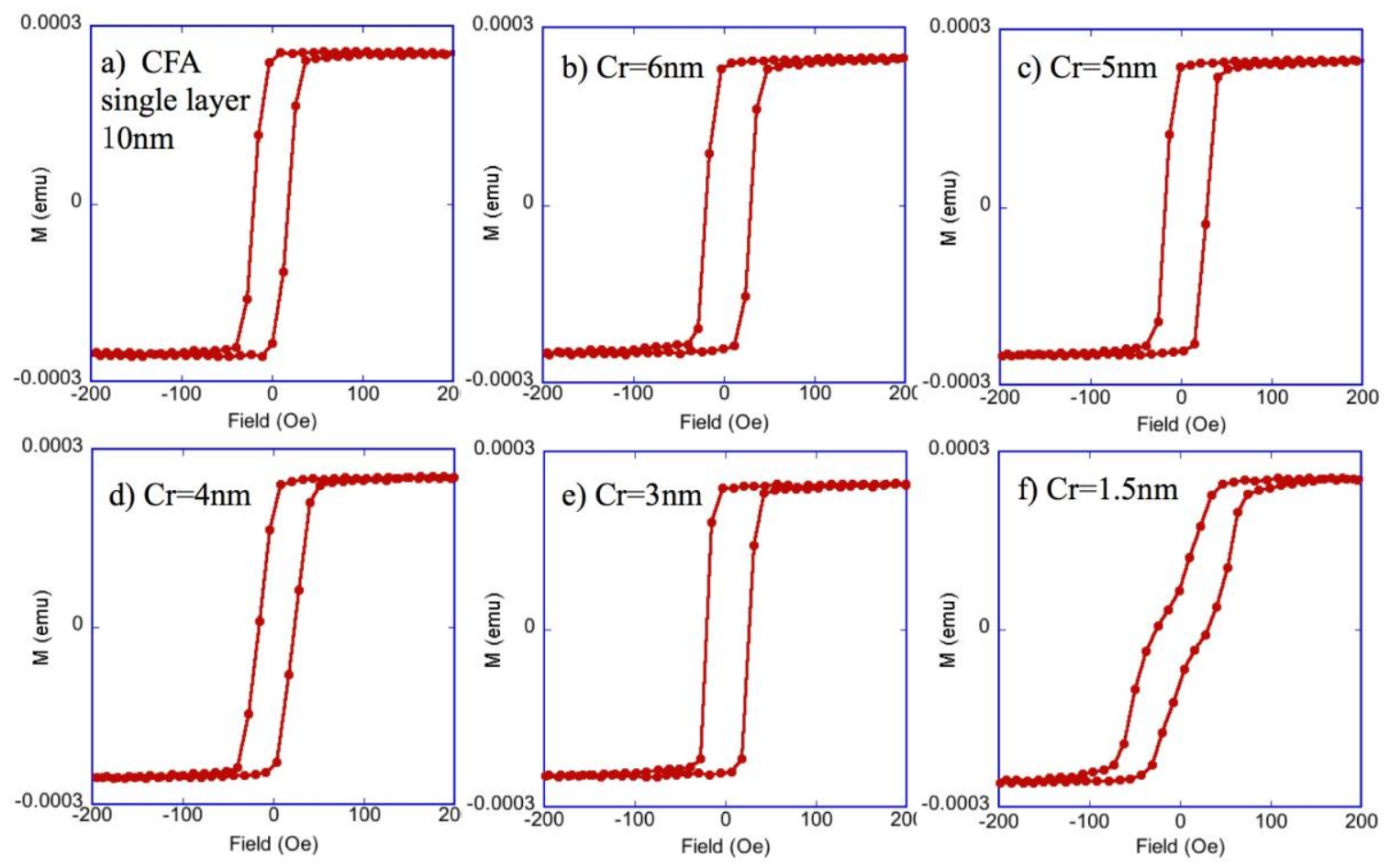

Figure 4.1 (a) Hysteresis loops of a single layer CFA layer with $\mathrm{t}=10 \mathrm{~nm}$. (b-f) Hysteresis loops for samples with different $\mathrm{Cr}$ thickness varied from $1.5 \mathrm{~nm}$ to $6 \mathrm{~nm}$. The samples were measured at room temperature. 


\subsubsection{Temperature dependent interlayer coupling in $\mathrm{CFA} / \mathrm{Cr} / \mathrm{CFA}$}

Samples with $\mathrm{Cr}$ spacer thickness of $1.5 \mathrm{~nm}$ are then used for the study of magneto transport in the pseudo spin-valve structure. Figure 4.2 shows the MR and the corresponding hysteresis loop measured at different temperature from $300 \mathrm{~K}$ to $50 \mathrm{~K}$.
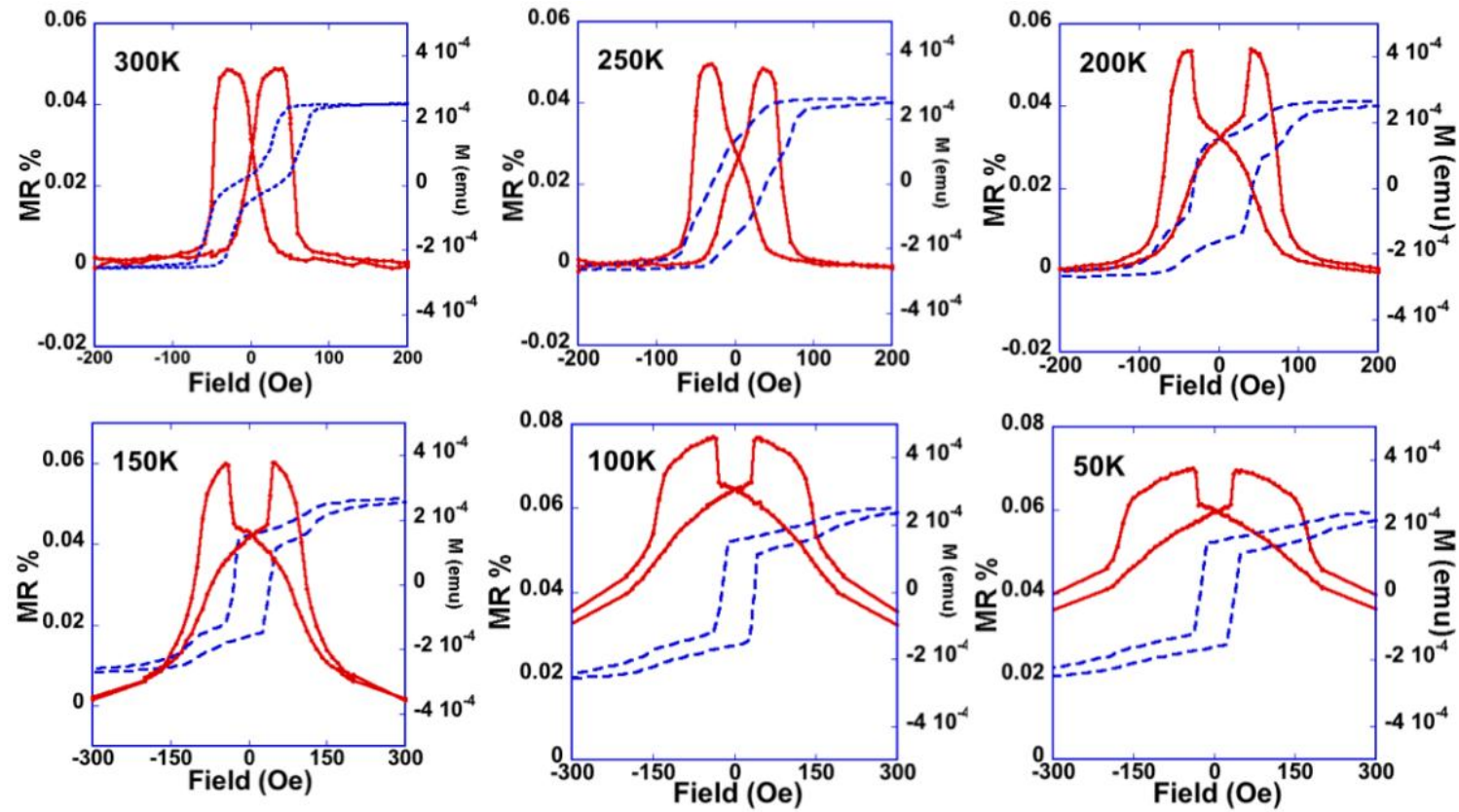

Figure 4.2 MR and the corresponding hysteresis loop of the trilayers with $\mathrm{Cr}$ spacer thickness of $1.5 \mathrm{~nm}$. The curves are measured at different temperature varying from $300 \mathrm{~K}$ to $50 \mathrm{~K}$.

To gain a better understanding of the MR behavior, I performed numerical simulation of the magnetic hysteresis loops to quantitatively describe the interlayer couplings. The magnetization curves for CFA/Cr/CFA trilayers have been simulated by using the total energy expression of $E_{T}=E_{K}+E_{Z}+E_{J}$, where the anisotropy energy $E_{K}$, the Zeeman 
energy $E_{Z}$, and exchange coupling energy $E_{J}$ can be expressed as follows [107]:

$$
\begin{gathered}
E_{K}=K_{a} t_{a} \sin ^{2} \alpha \cos ^{2} \alpha+K_{b} t_{b} \sin ^{2} \beta \cos ^{2} \beta \\
E_{Z}=-M_{a} t_{a} H \cos \alpha-M_{b} t_{b} H \cos \beta \\
E_{J}=-J_{1} \cos (\alpha-\beta)-J_{2} \cos ^{2}(\alpha-\beta)
\end{gathered}
$$

$K, t$, and $M$ are the first order cubic magnetocrystalline anisotropy energy constant, the thickness of CFA layers, and the saturation magnetization, respectively. The subscripts $a$ and $b$ represent the bottom and top CFA layers, respectively. $J_{1}$ and $J_{2}$ are the exchange constants of the first two terms of the general expansion of exchange coupling energy, which are known as the bilinear and biquadratic coupling parameters. A positive $J_{l}$ leads to a ferromagnetic coupling state, while negative $J_{1}$ and $J_{2}$ correspond to the $180^{\circ}$ and $90^{\circ}$ coupling configuration, respectively. $H$ is the applied magnetic field and a (or b) represents the in-plane angle between the direction of $H$ and $M$ of the bottom (or top) CFA layer in plane. $M-H$ loops of a CFA/Cr/CFA trilayer have been simulated by minimizing the total energy $E_{T} . M, t$, and $K$ are $1000 \mathrm{emu} / \mathrm{cm}^{3}, 5 \mathrm{~nm}$, and $3 \times 10^{3} \mathrm{erg} / \mathrm{cm}^{3}$ respectively, which can be obtained from experiments. Note this model assumes coherent, single domain switching of $\mathrm{Co}_{2} \mathrm{FeAl}$.

The red lines in Figure 4.3(a) represented the simulated $M-H$ curves assuming the bilinear coupling $J_{l}$ is constant at $-0.006 \mathrm{erg} / \mathrm{cm}^{3}$. The major feature of the experimental magnetization curves was reproduced in the calculated curves, in spite of the deviation of the coercive field due to the single domain assumption. Figure 4.3(b) showed the simulated 
magnitude $-J_{2}$ as a function of the temperature. A similar trend was also observed in $\mathrm{Fe} / \mathrm{Cr} / \mathrm{Fe}$ and other systems [108-110]. According to Figure 4.3(b), the strength of the bilinear antiferromagnetic coupling $J_{l}$ was comparable to the strength of the biquadratic coupling $J_{2}$ at $300 \mathrm{~K}$. As the temperature was reduced to/below $250 \mathrm{~K}$, the biquadratic coupling became dominant and increased significantly at low temperature.

In Ref.[109], it was shown that the Néel temperature of $\mathrm{Cr}$ would be reduced significantly as the film thickness decreased. As the thicknesses decreased below $5 \mathrm{~nm}, \mathrm{Cr}$ transformed from an antiferromagnetic ordering to a paramagnetic ordering [109]. Therefore, the biquadratic coupling cannot be related to the antiferromagnetic ordering in Cr. However, the near-linear increase of the biquadratic coupling strength with decreasing temperature indicates that the origin of the biquadratic coupling could be described using the thickness fluctuation model which creates biquadratic coupling through a combination of interface roughness and intra-layer ferromagnetic exchange [111]. 


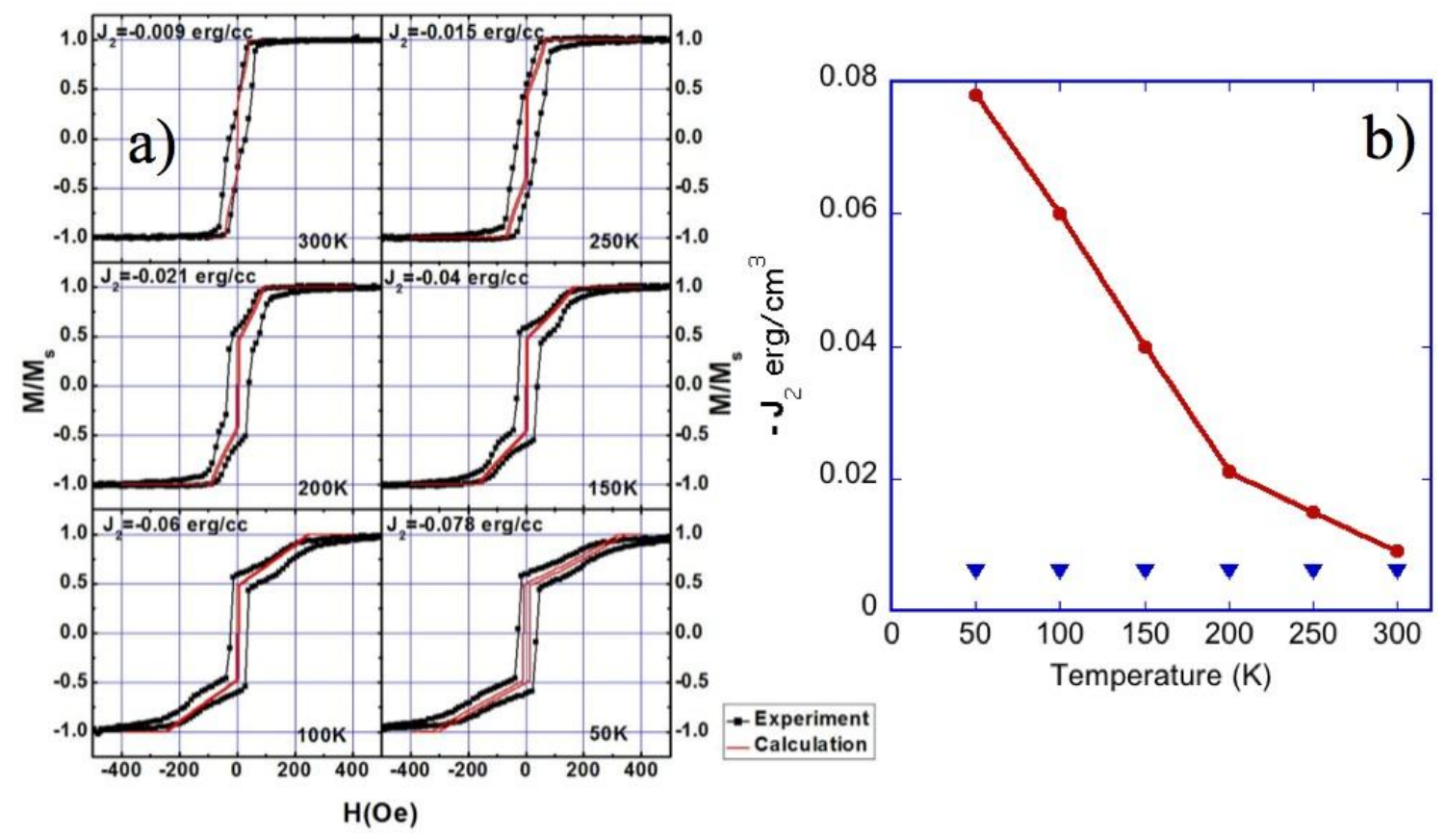

Figure 4.3 (a) Experimental (black line with squares) and calculated hysteresis loops (red line) of the trilayer with $\mathrm{Cr}$ thickness $1.5 \mathrm{~nm}$ measured at different temperatures from $300 \mathrm{~K}$ to $50 \mathrm{~K}$. $J_{l}$ is assumed as a constant of $-0.006 \mathrm{erg} / \mathrm{cm}^{3}$. (b) Calculated $-J_{2}$ as a function of the measurement temperature. The blue triangles indicate the value for $-J_{l}$.

To study the impact of $J_{l}$ and $J_{2}$ on the magnetization and the saturation field, the remanence $M_{r} / M_{s}$ ratio and $H s$ were plotted as a function of temperature as shown in Figure 4.4. The saturated field $H s$ increased up to 305 Oe at $50 \mathrm{~K}$. The remanence $M_{r} / M_{s}$ ratio increased to a maximum value of $\sim 0.58$ as the temperature decreased and almost kept constant as the temperature further decreased beyond $250 \mathrm{~K}$. The behavior for $M_{r} / M_{s}$ ratio and $H s$ agreed well with the trend for $J_{1}$ and $J_{2}$ as demonstrated in Figure 4.3(b). At 300K, the contribution from the bilinear antiferromagnetic coupling $J_{1}$ and the biquadratic 
coupling $J_{2}$ was comparable leading to a coupling close to $180^{\circ}$, and thus the $M_{t} / M_{s}$ ratio was close to 0 . However, as the temperature was reduced to/below $250 \mathrm{~K}$, the contribution of the biquadratic coupling became dominant, and thus the constant $M_{r} / M_{s}$ becoming close to the value of $0.5[112,113]$. As for $H s$, its magnitude increased to overcome the increasing strength of biquadratic coupling so that the two ferromagnetic layers can be aligned parallel with each other [112].

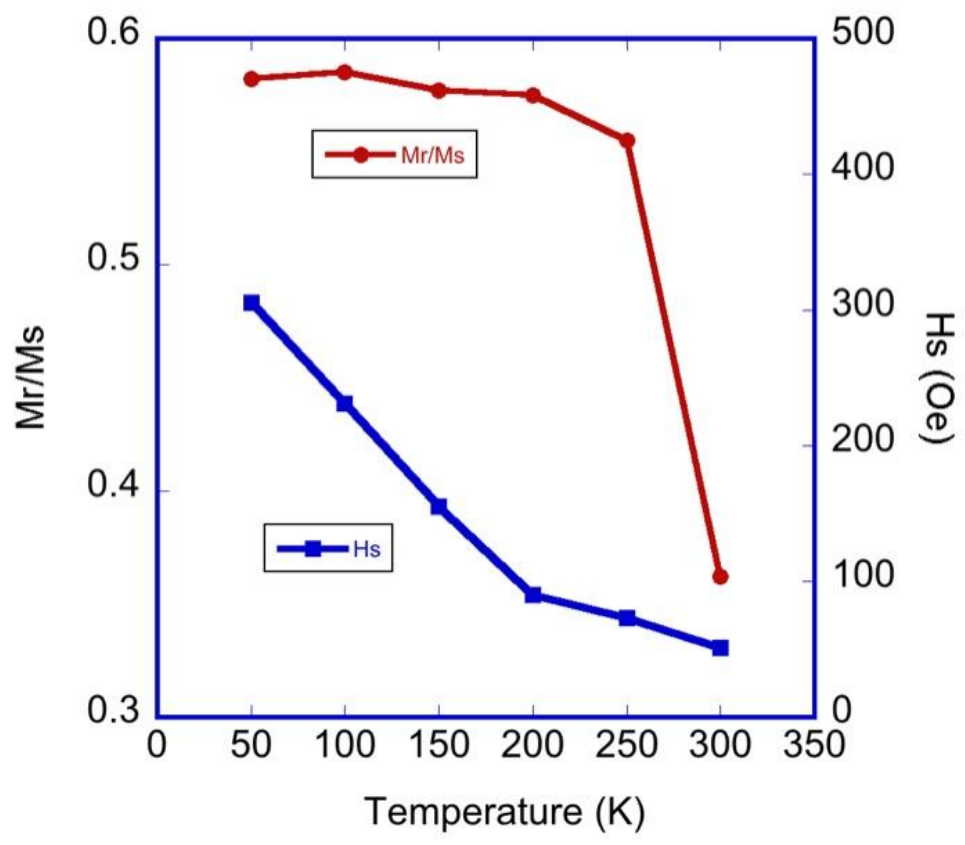

Figure 4.4 For the sample with a $\mathrm{Cr}$ thickness of $1.5 \mathrm{~nm}$, the experimental determined remanence $M_{r} / M_{s}$ ratio and $H s$ plotted as a function of temperature from $300 \mathrm{~K}$ to $50 \mathrm{~K}$.

\subsubsection{Magnetic alignments and MR ratio}

The numerical simulation helps to better understand the magnetic alignment states at 
different fields on the MR curves. For example, at $300 \mathrm{~K}$ [Figure 4.5(a)], the simulation indicated a negative value for both $J_{1}$ and $J_{2}$ with comparable strength and this led to an antiferromagnetic coupling. Experimentally, two plateaus were observed in the region between $-15 \mathrm{Oe}$ (or $15 \mathrm{Oe}$ ) to $50 \mathrm{Oe}$ (or $-50 \mathrm{Oe}$ ) corresponding to the antiparallel alignment of the two layer's magnetization. A field larger than 50 Oe saturates both layers and leads to a parallel alignment. At $50 \mathrm{~K}$ [Figure 4.5(b)], the biquadratic coupling became dominant leading to intermediate states(not completely parallel or anti-parallel). The gradual increase of MR from -200 Oe (or 200 Oe) to $30 \mathrm{Oe}$ (or -30 Oe) corresponded to the rotation of the top layer magnetization from state \#1 to a near $90^{\circ}$ position(state \#2) with respect to the bottom layer's magnetization. And the step corresponds to the sharp change from the $90^{\circ}$ position(\#2) to an antiparallel position(\#3).
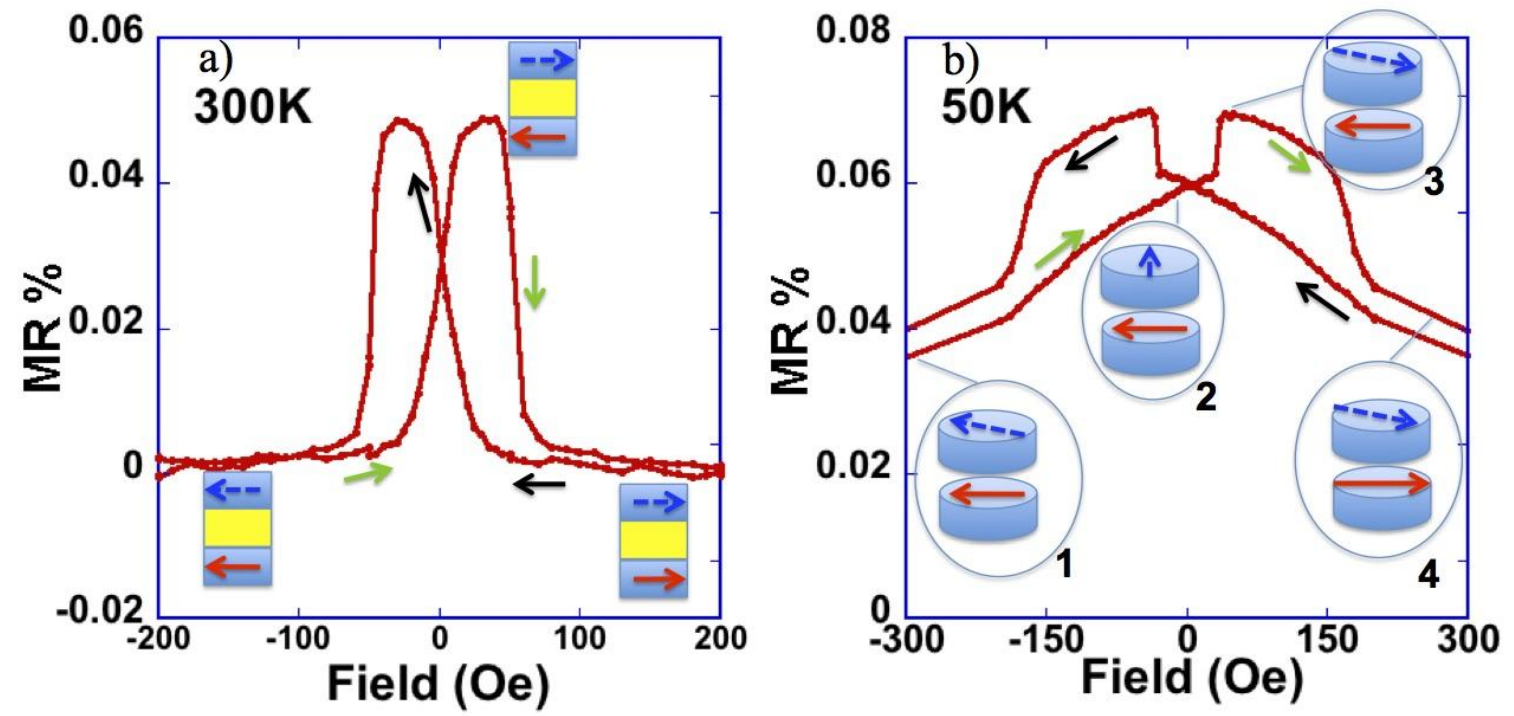

Figure 4.5 MR for the trilayer sample measured at room temperature $(300 \mathrm{~K})$ and $50 \mathrm{~K}$. The $\mathrm{Cr}$ spacer thickness is $1.5 \mathrm{~nm}$ for the sample. The black and green arrows indicate the 
field sweeping direction. The schematic diagrams illustrate the corresponding magnetic states (states \#1 to \#4) for different magnetoresistance regime on the MR curve guided by green arrows.

\subsection{CFA/Cu/CFA structure}

$\mathrm{Cu}$ has been commonly used as a spacer material due to its large spin diffusion length at room temperature $\sim 170 \mathrm{~nm}$ [114]. In order to improve the MR ratio of the CFA based pseudo spin-valves, I replaced $\mathrm{Cr}$ spacer with $\mathrm{Cu}$. The multilayers structures of $\mathrm{Ru}(20 \mathrm{~nm})$ $/ \mathrm{CFA}(3.5 \mathrm{~nm}) / \mathrm{Cu}(\mathrm{x}) / \mathrm{CFA}(3.5 \mathrm{~nm}) / \mathrm{Ru}(3 \mathrm{~nm})$ were deposited on $\mathrm{MgO}(001)$ substrates with $\mathrm{Cu}$ spacer thickness in the range of $3 \mathrm{~nm}$ to $10 \mathrm{~nm}$.

Figure 4.6 shows the comparison of hysteresis loops for two CFA samples with and without the Ru buffer layer. It turned out that the insertion of a Ru buffer layer can significantly enhance the $H_{c}$ of CFA from 25 Oe to 90 Oe. The increase of coercivity cannot be attributed to the surface roughness. The roughness of the two samples was measured by AFM as shown in Figure 4.7. The two samples have a small RMS roughness very close to each other indicating a very smooth Ru buffer layer for both layers.

The coercivity enhancement is possibly related to the strain effect caused by the lattice

mismatch. The lattice mismatch between the lattice parameters $\mathrm{a}_{\mathrm{CFA}} / \sqrt{2}$ and $\mathrm{a}_{\mathrm{MgO}}$ is small $\sim 3.7 \%$ [115] compared with the lattice mismatch between the CFA/Ru interface $\sim 8 \%$ and 
$\mathrm{MgO} / \mathrm{Ru}$ interface $\sim 11 \%$ [116]. The presence of lattice mismatch induced strain may give rise to the magneto-elastic anisotropy and therefore lead to the enhancement of the coercivity [117], yet more work need to be done to understand the intrinsic mechanism for the increase of coercivity.

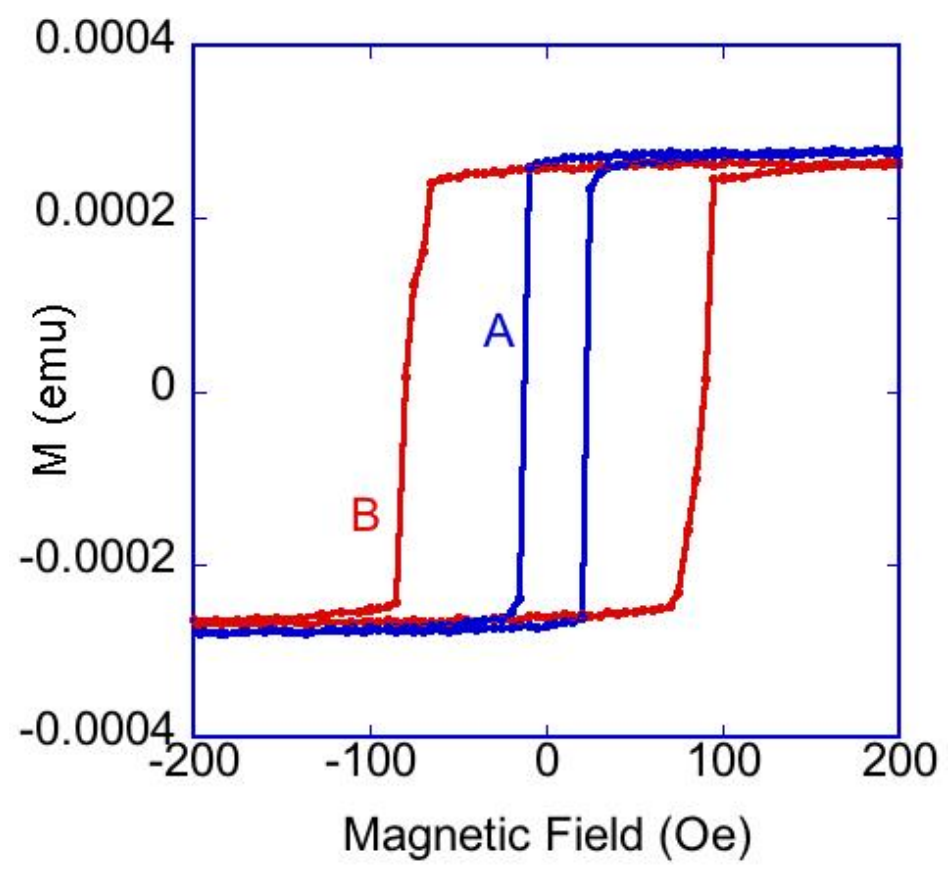

Figure 4.6 The hysteresis loops for sample A and B, corresponding to the structure $\mathrm{MgO}$ (substrate)// $\mathrm{CFA}(11 \mathrm{~nm})$ and $\mathrm{MgO}$ (substrate)// $\mathrm{Ru}(20 \mathrm{~nm}) / \mathrm{CFA}(11 \mathrm{~nm})$ respectively. 


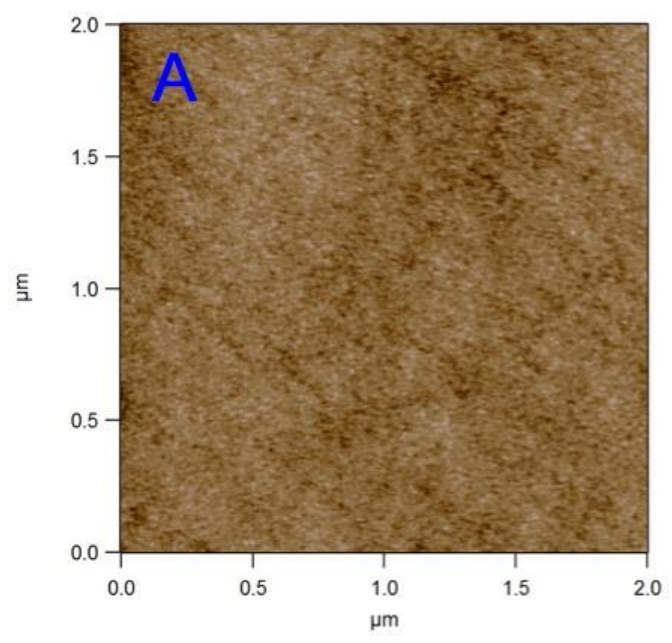

$\mathrm{RMS}=0.089 \mathrm{~nm}$

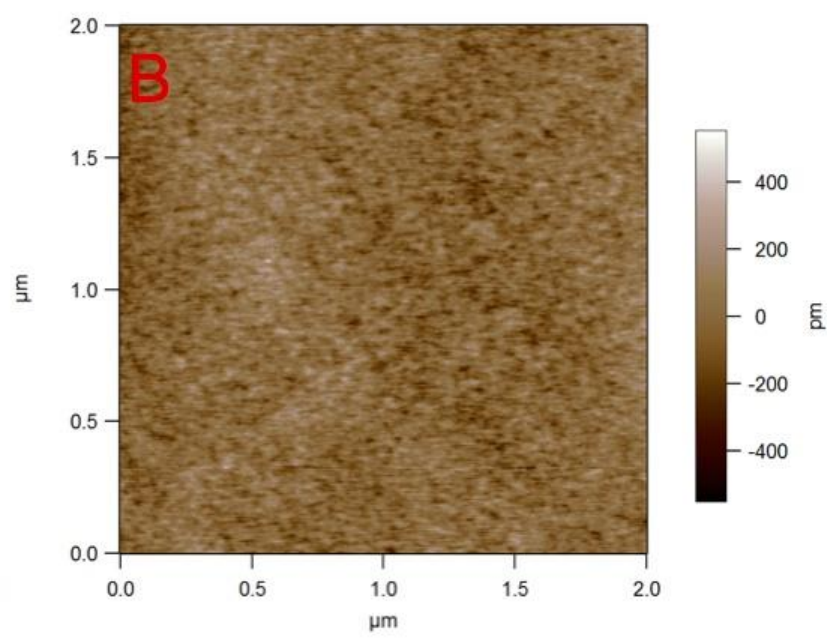

$\mathrm{RMS}=0.093 \mathrm{~nm}$

Figure 4.7 AFM image showing the topography of sample A and B. The roughness is given by the RMS value.

\subsubsection{Interlayer magnetic coupling}

The hysteresis loops of $\mathrm{CFA} / \mathrm{Cu}(\mathrm{x}) / \mathrm{CFA}$ with different $\mathrm{Cu}$ spacer thicknesses were shown in Figure 4.8. The two CFA layers were ferromagneticlly coupled with each other with $\mathrm{Cu}=3 \mathrm{~nm}$ and showed sign of separation from coupling beyond a $\mathrm{Cu}$ thickness of 5 nm. Unlike the trilayer with a $\mathrm{Cr}$ spacer, there was no antiferromagnetic coupling at room temperature. According to the AFM measurement, the CFA3.5nm/Cu surface roughness increased with the increase of $\mathrm{Cu}$ thickness, so samples with $\mathrm{Cu}=5 \mathrm{~nm}$ were used for the follow-on study. 


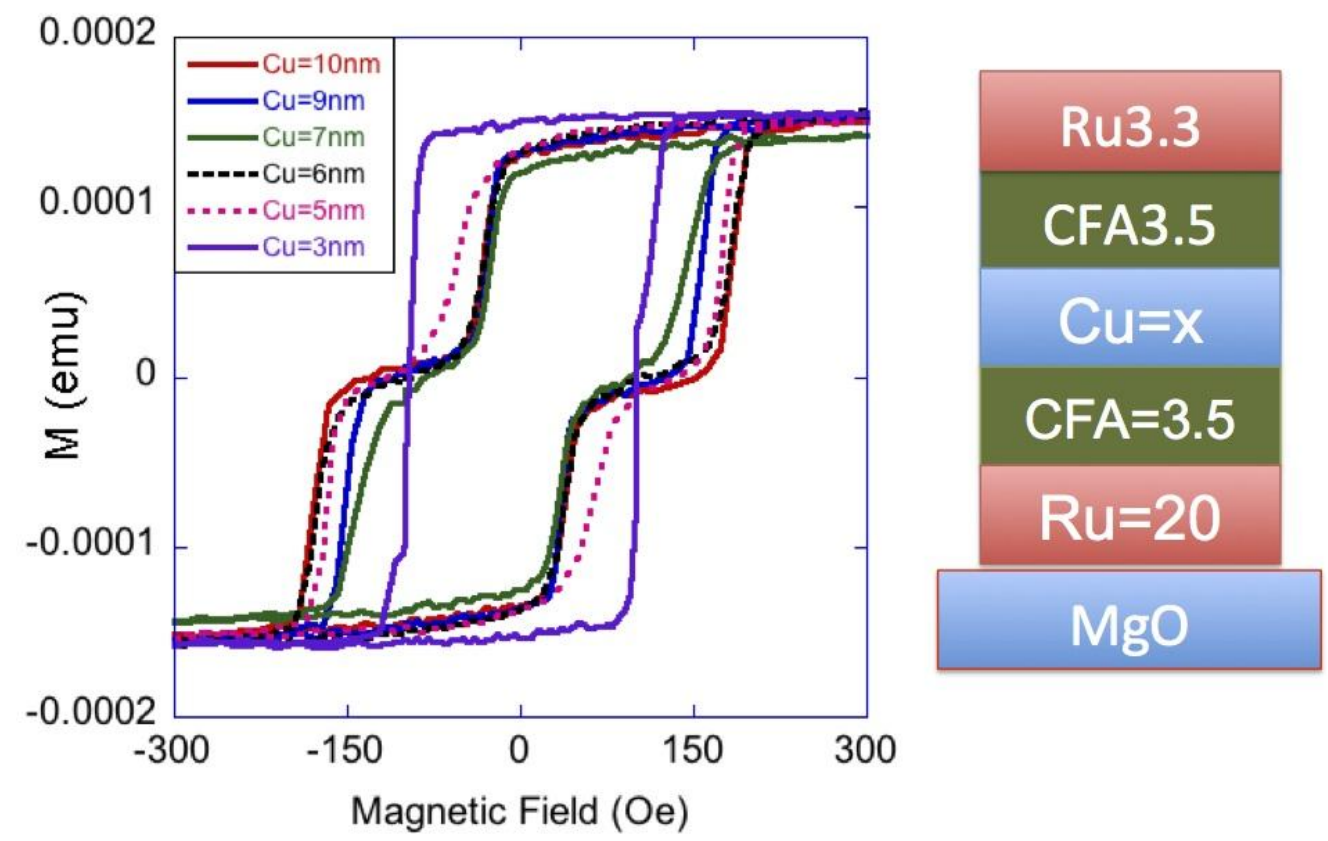

Figure 4.8 The hysteresis loops for samples with different $\mathrm{Cu}$ spacer thicknesses were measured at room temperature. The structure for the sample was $\mathrm{MgO}$ (substrate)// $\mathrm{Ru}(20 \mathrm{~nm}) / \mathrm{CFA}(3.5 \mathrm{~nm}) / \mathrm{Cu}(\mathrm{x}) / \mathrm{CFA}(3.5 \mathrm{~nm}) / \mathrm{Ru}(3 \mathrm{~nm})$.

\subsubsection{MR ratio for $\mathrm{CFA} / \mathrm{Cu} / \mathrm{CFA}$ trilayers}

A very small $\mathrm{MR}$ ratio was observed on the $\mathrm{CFA} / \mathrm{Cu}(5 \mathrm{~nm}) / \mathrm{CFA}$ as-deposited sample as shown in Figure 4.9(a). At room temperature, the MR ratio is $\sim 0.02 \%$, which is much smaller than the value observed on the CFA/Cr1.5/CFA sample. We suspected the low chemical ordering of the as-deposited CFA layer might affect the spin-polarization. To improve the chemical ordering, annealing was carried out using a RTA at $350{ }^{\circ} \mathrm{C}$ for 20 minutes. The MR ratio increased to $\sim 0.04 \%$ after annealing as shown in Figure 4.9(c). 
Higher temperature annealing at $450{ }^{\circ} \mathrm{C}$ deteriorated the GMR performance due to the promotion of interlayer diffusion between the $\mathrm{CFA} / \mathrm{Cu}$ interfaces.
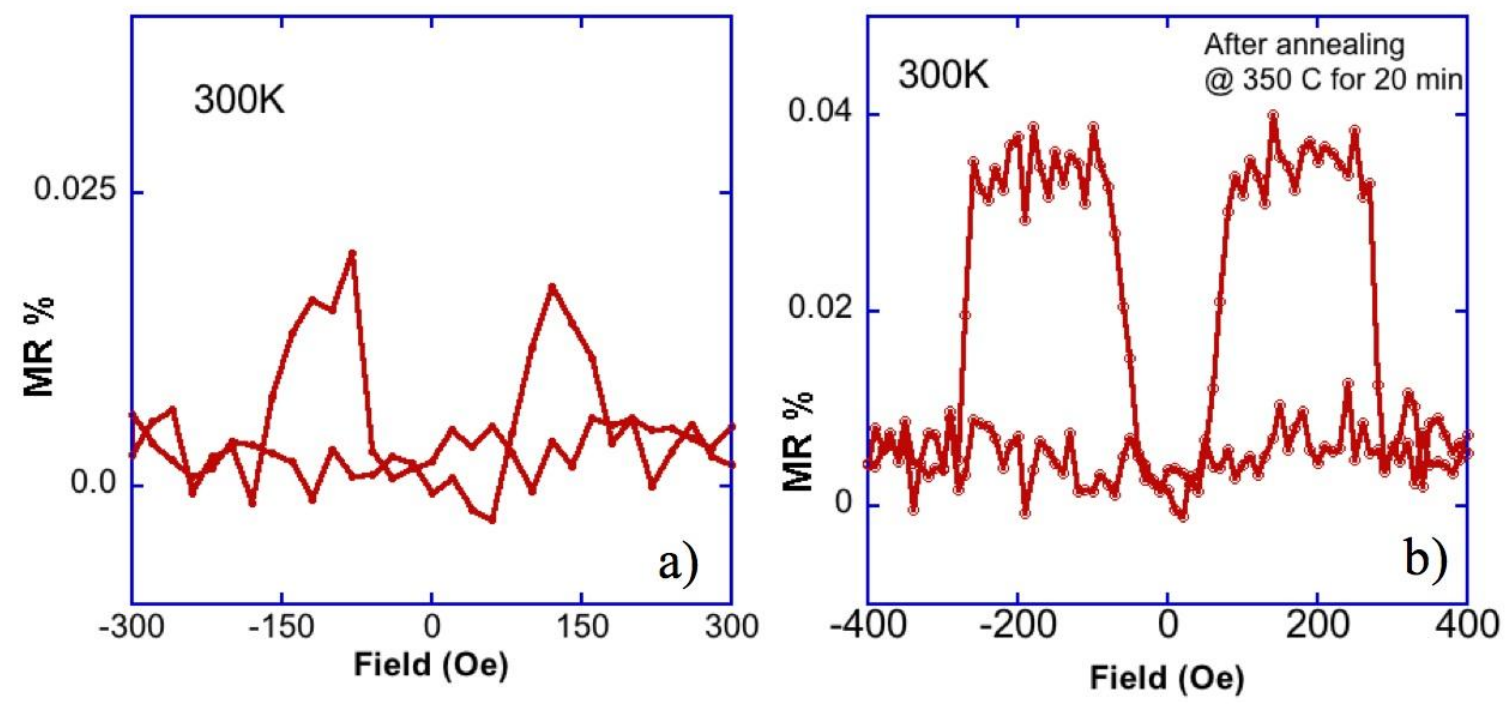

Figure 4.9 MR for the CFA/Cu5/CFA sample before \& after annealing measured at 300K.

Magnetic tunnel junction structure of $\mathrm{CFA} / \mathrm{MgO} / \mathrm{CoFe} / \mathrm{IrMn}$ had been reported to have TMR ratio as high as $330 \%$ at room temperature. The high TMR ratio was ascribed to the epitaxial growth of the CFA layer on $\mathrm{Cr}$ buffered $\mathrm{MgO}$ substrate that led to an ideal B2 structure of CFA [118]. Our previous work also revealed a spin-polarization ratio of $\sim 54 \%$ at $4.2 \mathrm{~K}$ for $50 \mathrm{~nm}$ CFA layer grew epitaxial on $\mathrm{MgO}(001)$ substrate [95]. However, the XRD results indicated the Ru buffered CFA/Cu/CFA structure did not have an epitaxial relationship and therefore the CFA layers did not have an ideal B2 structure, which was necessary for obtaining a high spin-polarization. Moreover, the top CFA layer was suggested to have an even smaller spin-polarization ratio than the bottom one due to the 
disordered crystalline structure and the building-up of surface roughness [119]. To improve, $\mathrm{Cr}$ buffer layer could be used for the $\mathrm{CFA} / \mathrm{Cu} / \mathrm{CFA}$ structure and a top pinning layer will be needed to achieve separation between the top and bottom CFA electrodes' coercive fields.

\subsubsection{CFA/Cu/CFA on $\mathrm{SiO}_{2}$ substrate}

In order to study the magnetoresistance by using the current perpendicular to plane (CPP) method, deposition of the multilayer structure on the $\mathrm{SiO}_{2}$ substrate was desired. To carry this out, new recipes were explored to deposit the $\mathrm{CFA} / \mathrm{Cu} / \mathrm{CFA}$ trilayer on $\mathrm{SiO}_{2}$ substrate. The magnetic separation between the two ferromagnetic layers is achieved by adjusting the thickness of the free layer. Figure 4.10(a) shows the hysteresis loop for the structure $\mathrm{SiO}_{2}$ (substrate)//Ru20(nm) $/ \mathrm{CFA}(3.5 \mathrm{~nm}) / \mathrm{Cu}(6 \mathrm{~nm}) / \mathrm{CFA}(1.7) / \mathrm{Ru}(3)$. A MR of $\sim 0.04 \%$ at $300 \mathrm{~K}$ was observed on the sample by Van der Pauw method on the continuous film. The change of substrate from $\mathrm{MgO}$ to $\mathrm{SiO}_{2}$ did not improve the MR ratio. As aforementioned, the successful establishment of B2 structure for CFA was crucial for a high spin-polarization and hence a high MR ratio. Further work to grow an epitaxial CFA electrode on $\mathrm{SiO}_{2}$ will be required to achieve a higher MR ratio. 

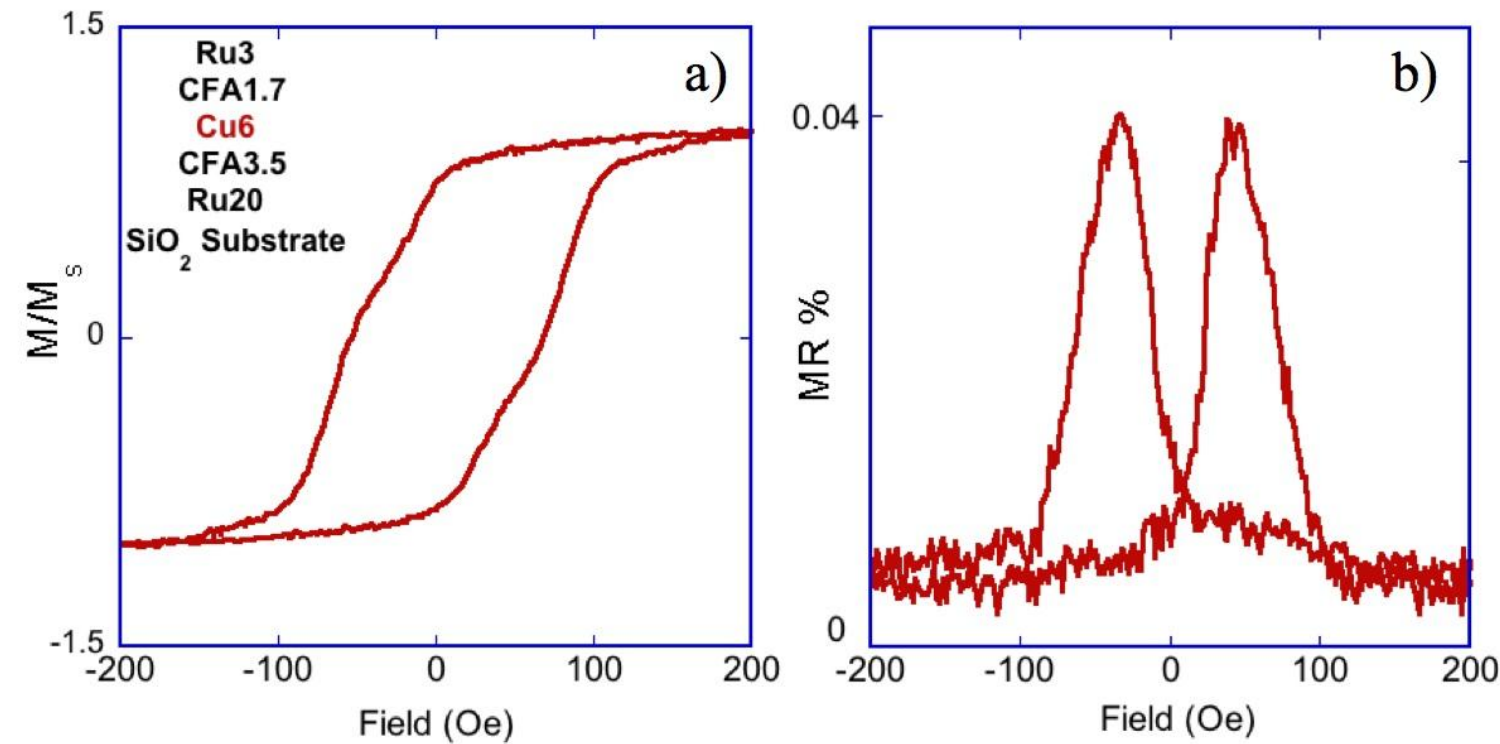

Figure 4.10 The hysteresis loop (a) and the corresponding MR curve (b) measured at room temperature. The sample was grew on $\mathrm{SiO}_{2}$ substrate with a structure $\mathrm{Ru} 20(\mathrm{~nm})$ / $\mathrm{Co}_{2} \mathrm{FeAl}(3.5 \mathrm{~nm}) / \mathrm{Cu}(6 \mathrm{~nm}) / \mathrm{Co}_{2} \mathrm{FeAl}(1.7) / \mathrm{Ru}(3)$

\subsection{CFA/Cu/CFA with nano-oxide layer}

\subsubsection{Nano-Oxide Layer}

Several approaches had been carried out to improve the MR ratio as aforementioned, however, none of them showed significant increase of MR for the PSV. It has been reported that Nano-oxide layers (NOL) inserted at the top surface of a spin valve could very effectively increase the MR ratio $[120,121]$. The enhancement of the MR was attributed to the specular reflection due to the presence of NOLs. The specular reflection of 
spin-polarized electrons from the oxide interface conserved the electrons' spin-direction and enhanced the spin-dependent scattering at the Ferromagnetic/Non-magnetic interface [122]. Several possible ways to enhance the specular reflection had been reported, such as depositing noble metals on top of SVs to obtain an atomic-smooth surface [123], using oxides as the pinning layer [124], or inserting a NOL to increase the potential barrier of electrons at the interfaces [120]. In the BTIBD system, a NOL can be introduced by bombarding the surface with an oxygen plasma, by nature oxidation in $\mathrm{Ar} / \mathrm{O}_{2}$ environment, or by reactive sputtering of a target material (Co in our case) with $\mathrm{O}_{2}$. To date, there are few reports on NOL based SVs or PSVs with full-Heusler alloy electrodes. In this section, I present the preliminary results of a significant enhancement of MR by inserting the NOL into the CFA based magnetic multilayers.

\subsubsection{CFA/Cu/CFA with NOL}

The film was deposited on a $\mathrm{SiO}_{2}$ substrate with a structure of $\mathrm{Ta}(10 \mathrm{~nm}) / \mathrm{CoO}(1.5 \mathrm{~nm})$ $/ \mathrm{Co}(0.5 \mathrm{~nm}) / \mathrm{CFA}(3.5 \mathrm{~nm}) / \mathrm{Cu}(8 \mathrm{~nm}) / \mathrm{CFA}(1.7 \mathrm{~nm}) / \mathrm{Ta}(5 \mathrm{~nm})$. The thickness of $\mathrm{CoO}$ and Co layer was estimated based on the deposition rate and oxidation time of Co layer. The thickness for the $\mathrm{Cu}$ spacer and the top CFA layer is optimized to balance between the interlayer coupling and surface roughness. The NOL layer, which is the $\mathrm{CoO}$, is formed by exposing a $\sim 1.5 \mathrm{~nm}$ Co layer to the $\mathrm{O}_{2}$ plasma $(\sim 100 \mathrm{~W})$ without breaking the vacuum. A very thin layer of $\mathrm{Co}$ is deposited on top of $\mathrm{CoO}$ to prevent the oxidation of the CFA layer. 
Annealing treatment was carried out for all samples listed in this section at $350{ }^{\circ} \mathrm{C}$ for $12 \mathrm{~s}$ at high vacuum using an RTA.

Bulk $\mathrm{CoO}$ is an antiferromagnetic material with an Néel temperature of $\sim 291 \mathrm{~K}$. It is noteworthy to mention that $\mathrm{CoO} / \mathrm{Co}$ interface tends to enhance the coercivity due to the strong exchange bias when the temperature is below the Néel temperature $[125,126]$. Table 4-1 shows the comparison of coercive fields at different temperatures. Significant coercivity enhancement was obtained when the temperature is lowered to/below $150 \mathrm{~K}$. According to Ref.[127], the exchange bias fields data can be fitted in a model $\mathrm{H}_{E}=$ $\mathrm{H}_{E O}\left(1-\mathrm{T} / \mathrm{T}_{B}\right)^{\mathrm{n}}$ with $\mathrm{T}<\mathrm{T}_{B}$, which resulted in $\mathrm{H}_{E O} \sim 6549 \mathrm{Oe}, \mathrm{T}_{B} \sim 187 \mathrm{~K}$ and $\mathrm{n} \sim 1.2$. The fact that the exchange bias occurred only below the blocking temperature $T_{B} \sim 2 / 3 T_{N}$ was quantitatively explained by the Stiles and McMichael model [128]. The $\mathrm{H}_{E O}$ obtained in the $\mathrm{CoO} / \mathrm{Co}(5 \AA) / \mathrm{CFA}(35 \AA)$ system was nearly an order larger than the one on $\mathrm{CoO} / \mathrm{Co}(160 \AA)$ system $\sim 500$ Oe [127]. The different behavior might be explained by the change of reversal mechanism from domain wall motion to coherent domain rotation, which had been predicted in certain bilayer systems and depended on the thickness and grain size of the FM layer $[125,129]$. Figure 4.11 shows a distinct magnetic separation between the two CFA layers at $150 \mathrm{~K}$, the one with larger coercive fields ( 925 Oe) corresponds to the CFA layer at the bottom. 


\begin{tabular}{|c|c|c|}
\hline $\begin{array}{c}H_{c} \text { at different } \\
\text { Temperature }\end{array}$ & $\begin{array}{c}\text { Ta/Co/CFA } \\
(\text { Oe })\end{array}$ & $\begin{array}{c}\text { Ta/CoO/Co/CFA } \\
(\text { Oe })\end{array}$ \\
\hline $300 \mathrm{~K}$ & 21.2 & 20.2 \\
\hline $250 \mathrm{~K}$ & 25.5 & 30.5 \\
\hline $200 \mathrm{~K}$ & 30.3 & 76.1 \\
\hline $150 \mathrm{~K}$ & 32.5 & 924.5 \\
\hline $100 \mathrm{~K}$ & 42.5 & 2328.5 \\
\hline $50 \mathrm{~K}$ & 53.7 & 4310.5 \\
\hline
\end{tabular}

Table 4-1 The comparison of the CFA coercive fields at different temperatures. Results for two interfaces with \& without NOL are compared.
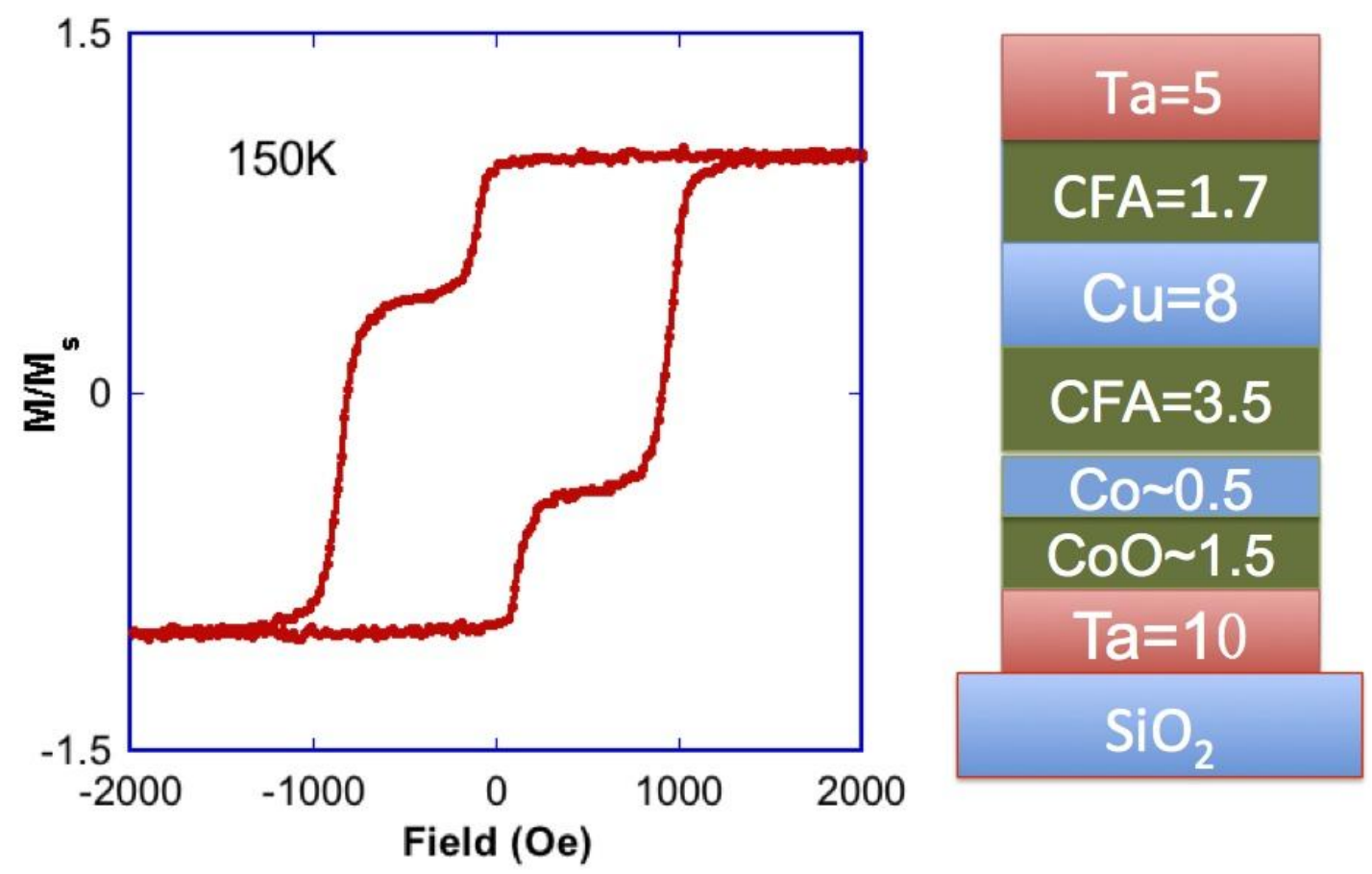

Field (Oe)

Figure 4.11 $M-H$ loop for the NOL/CFA/Cu/CFA sample measured at $150 \mathrm{~K}$. 


\subsubsection{Enhancement of MR ratio by NOL}

The MR ratio observed on the NOL/CFA/Cu/CFA structure is $\sim 0.8 \%$ at $150 \mathrm{~K}$. The MR ratio with the NOL is more than an order of magnitude higher than the one observed on $\mathrm{CFA} / \mathrm{Cr} / \mathrm{CFA}(0.06 \%$ at $150 \mathrm{~K})$ and on $\mathrm{CFA} / \mathrm{Cu} / \mathrm{CFA}(0.05 \%$ at $150 \mathrm{~K})$. Figure 4.12 shows the plateau region corresponding to an antiparallel alignment between $\sim 100$ Oe to $\sim 900$ Oe, which agrees with the hysteresis loop as shown in Figure 4.11. A broad plateau regime is also observed due to the large enhancement of coercive field by the $\mathrm{CoO}$ layer.

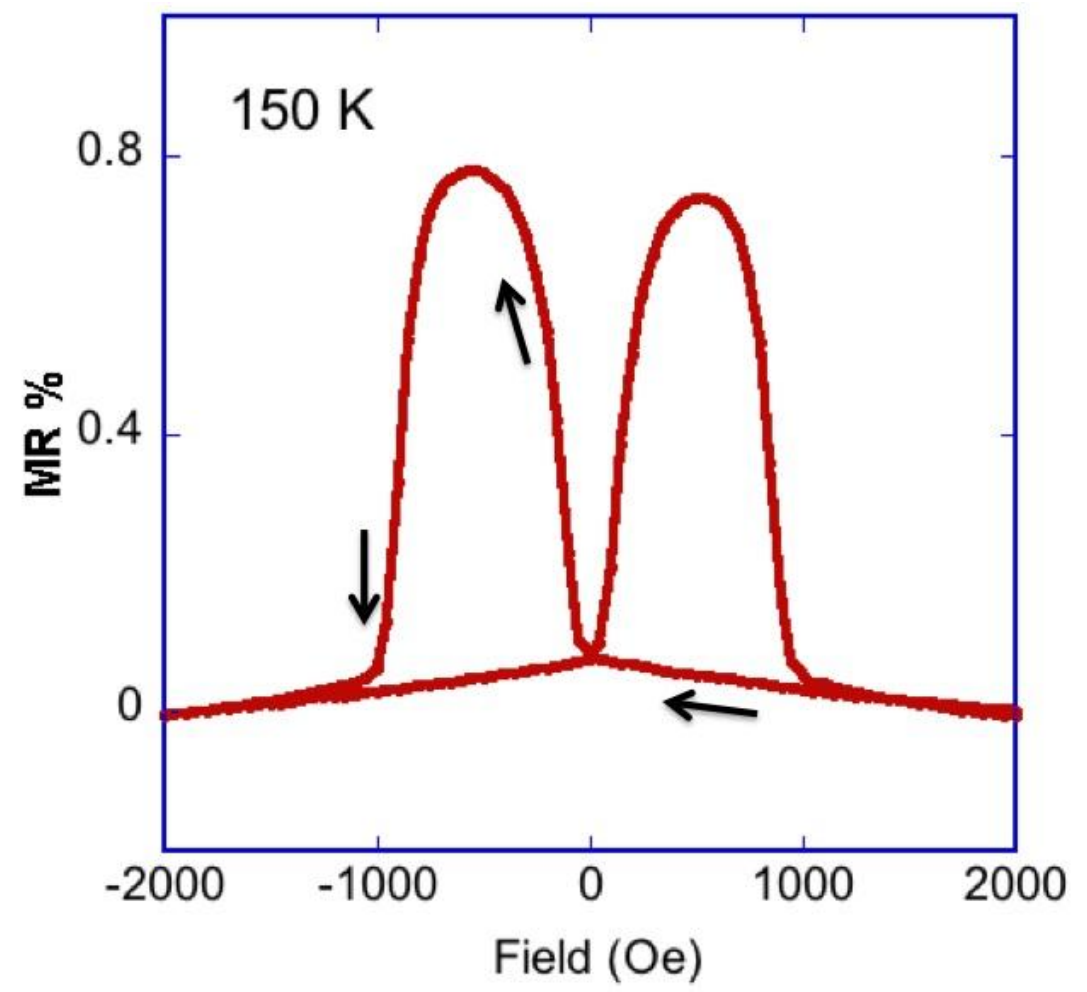

Figure 4.12 MR for the NOL/CFA/Cu/CFA sample measured at $150 \mathrm{~K}$. The arrows indicate the field sweeping direction. The sample was annealed at $350{ }^{\circ} \mathrm{C}$ for 12 seconds under high vacuum. 
The classic two-current series resistor model for CPP-GMR assumes no spin-flipping (or infinite spin-diffusion length) during the propagation of the electrons through the trilayer. However, according to Ref.[130], a finite spin-diffusion length $\left(l_{\mathrm{sf}}\right)$ will affect the MR ratio once $l_{\mathrm{sf}}<<t_{\mathrm{F}}, \mathrm{t}_{\mathrm{F}}$ is the ferromagnetic layer film thickness. Under such a scenario, the electrons only conserve their spin-direction for a very short distance during the propagation and therefore the spin-dependent scattering at the N/F interface is reduced. Figure 4.13 schematically shows the spin-flipping of an electron during the drift in a conductive material. For CIP-GMR, the scaling length is the relatively short electron mean free path (MFP). The experimental results show that the $l_{\text {sf }}$ of $\mathrm{Co}_{90} \mathrm{Fe}_{10}$ is about $25 \mathrm{~nm}$ [131] and for CoFeAlSi is about 3nm [132]. CFA has the same structure as CoFeAlSi except that half of the $\mathrm{Al}$ atoms are substituted by $\mathrm{Si}$ atoms; hence the MFPs of CFA and CFAS should be very close and smaller than $3 \mathrm{~nm}$. The small MFP is likely to severely affect the MR ratio on the CFA based PSVs. For current in plane measurements, the NOLs extend the MFP for the spin-polarized electrons through specular reflection at the F/NOL interface and enhance the MR ratio. 


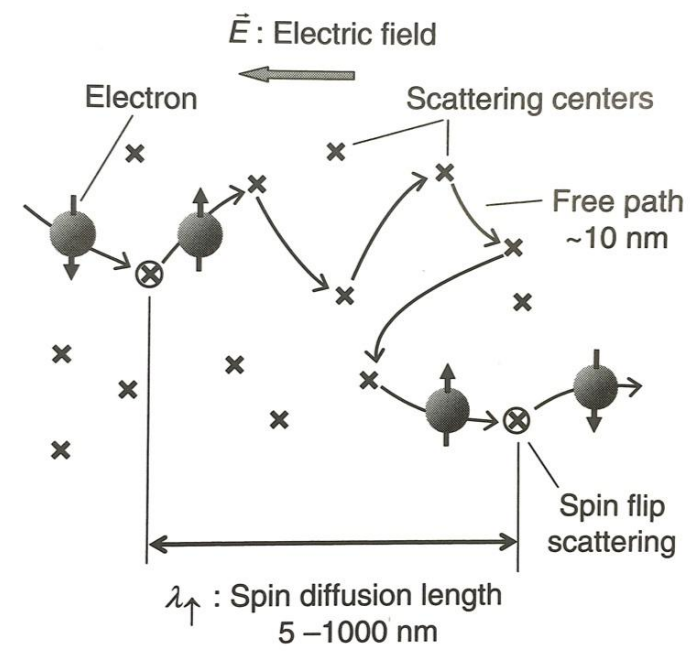

Figure 4.13 Schematic drawing of the spin diffusion length. During the propagation of the electron, the electron moves opposite to the electric field $E$ on average. The average distance between two scattering events is the mean free path. For metals at room temperature, the typical mean free path is about $10 \mathrm{~nm}$. The average distance between two spin-flip events corresponds to the spin diffusion length $l_{\text {sf }}$. (Adpted from Ref. [133])

\subsection{Summary}

In this chapter I studied the static magnetic and magneto transport properties of $\mathrm{Co}_{2} \mathrm{FeAl}$ based magnetic pseudo spin-valve (PSV) structures fabricated by BTIBD. Without using an antiferromagnetic pinning layer, several possible ways were introduced to achieve a magnetic separation for the PSV, such as by antiferromagnetic coupling, utilizing different seeding layer, by adjusting the ferromagnetic layer thickness, or by inserting a $\mathrm{CoO} \mathrm{NOL}$. 
Starting from the CFA/Cr/CFA trilayers, I studied the temperature dependent interlayer coupling. Numerical simulation was performed to understand the transition from $180^{\circ}$ coupling to $90^{\circ}$ coupling, which also explained the different magnetic states on the MR curve. A very small MR ratio was observed at room temperature of $\sim 0.05 \%$, and the MR increased to only $\sim 0.07 \%$ at $50 \mathrm{~K}$ for the CFA/Cr/CFA structure. As introduced in Chapter 1, the output power of STNO would be proportional to (MR ${ }^{2}$. Therefore, a small MR ratio will impede its application as a spin-torque oscillator. Additionally, optimizations were made by changing the spacer to $\mathrm{Cu}$ in $\mathrm{CFA} / \mathrm{Cu} / \mathrm{CFAPSV}$ and by adding a nano-oxide layer in NOL/CFA/Cu/CFA structure to improve the MR ratio. A large enhancement of MR ratio was observed in the optimized $\mathrm{NOL} / \mathrm{CFA} / \mathrm{Cu} / \mathrm{CFA}$ structure due to the specular reflection from the NOL. The results for the NOL structure shows a promising trend to increase the MR ratio, however further optimization is necessary for room temperature MR enhancement. Further improvement of the MR ratio will include the epitaxial deposition of the CFA electrode on different substrates and the optimization of the CFA crystalline in the multilayer structures. 


\section{Chapter $5 \mathrm{L1}_{0} \mathrm{MnAl}$ and magnetic multilayers based on MnAl}

\subsection{Introduction}

In this Chapter, $\mathrm{L1}_{0} \mathrm{MnAl}$ 's magneto-transport properties as a function of temperature are investigated using a Hall bar structure. In addition, the magnetic domain structures were characterized by magnetic force microscope (MFM) and the impact of domain wall scattering on magneto-resistance was analyzed. The DW effect studied here sheds light on understanding the spin-transfer on large size nano-pillars with inhomogeneities, which will be discussed in Chapter 7. Moreover, $\mathrm{L}_{0} \mathrm{MnAl}$ based magnetic hybrid structure is explored for the purpose of spin transfer study.

\subsection{Epitaxial of $\mathrm{L1}_{0} \mathrm{MnAl}$ film}

\subsubsection{Structure and magnetic properties of epitaxy MnAl}

The structural properties of MnAl films were characterized by the XRD $2 \theta$ scan as shown in Figure 5.1. The peak at $42.92^{\circ}$ corresponds to the diffraction of the $\mathrm{MgO}$ substrate. The peak at $64.34^{\circ}$ was due to the diffraction of (002) $\mathrm{Cr}$ planes indicating the bcc crystallization of the $\mathrm{Cr}$ layer. In addition to the diffraction peaks of the $\mathrm{MgO}$ substrate 
and $\mathrm{Cr}$ seed layer, two diffraction peaks of $\mathrm{MnAl}$ were observed at $25.21^{\circ}$ and $51.59^{\circ}$, corresponding to the (001) peak and (002) peak respectively. The appearance of the (002) peak implied the presence of the tetragonal body centered lattice in the film, regardless of the chemical arrangement of $\mathrm{Mn}$ and $\mathrm{Al}$. The superlattice (001) peak indicated the presence of a B2 structure of $\mathrm{MnAl}$, an alternative ordering between the $\mathrm{Mn}$ and $\mathrm{Al}$ atomic planes along the [001] direction, as shown in Figure 5.1 inset. The order parameter $S$ for the MnAl was estimated to be $\sim 0.89$ using integrated intensities of the (001) and (002) peaks[134]. This indicated a very high degree of chemical ordering in the MnAl thin film ( $S=1$ represents perfect chemical ordering [134]).

$\Phi$ scans were similarly taken with $2 \theta$ positions corresponding to the $\tau$-MnAl (011), $\mathrm{Cr}(011)$ and $\mathrm{MgO}(022)$ peaks to confirm the epitaxial growth of each layer (Figure 5.2). In this case, an in-plane $45^{\circ}$ orientation offset occurred between the (001) $\mathrm{Cr}$ and $(001) \mathrm{MgO}$ planes while planes (110) $\mathrm{Cr} / /(100) \mathrm{MgO}$ along the $c$-axis. On the other hand, the $\tau$-MnAl grew on the $\mathrm{Cr}$ layer coherently with planes (001) $\tau$-MnAl//(001)Cr and (100) $\tau-\mathrm{MnAl} / /(100) \mathrm{Cr}$. The schematic diagram for the described lattice matching is presented in Figure 5.2. From the $2 \theta$ values of the MnAl (001) and (011) peaks, one can use Bragg's Law to calculate lattice constants $a=2.78 \AA$ (in plane) and $c=3.53 \AA$ (out of plane) with c/a $\sim 1.27$, which suggested a very good tetragonal distortion in comparison with bulk $\mathrm{L}_{0}$ $\operatorname{MnAl}(\mathrm{a}=2.77 \AA$ and $\mathrm{c}=3.57 \AA$ with $\mathrm{c} / \mathrm{a}=1.29)$ [83]. 


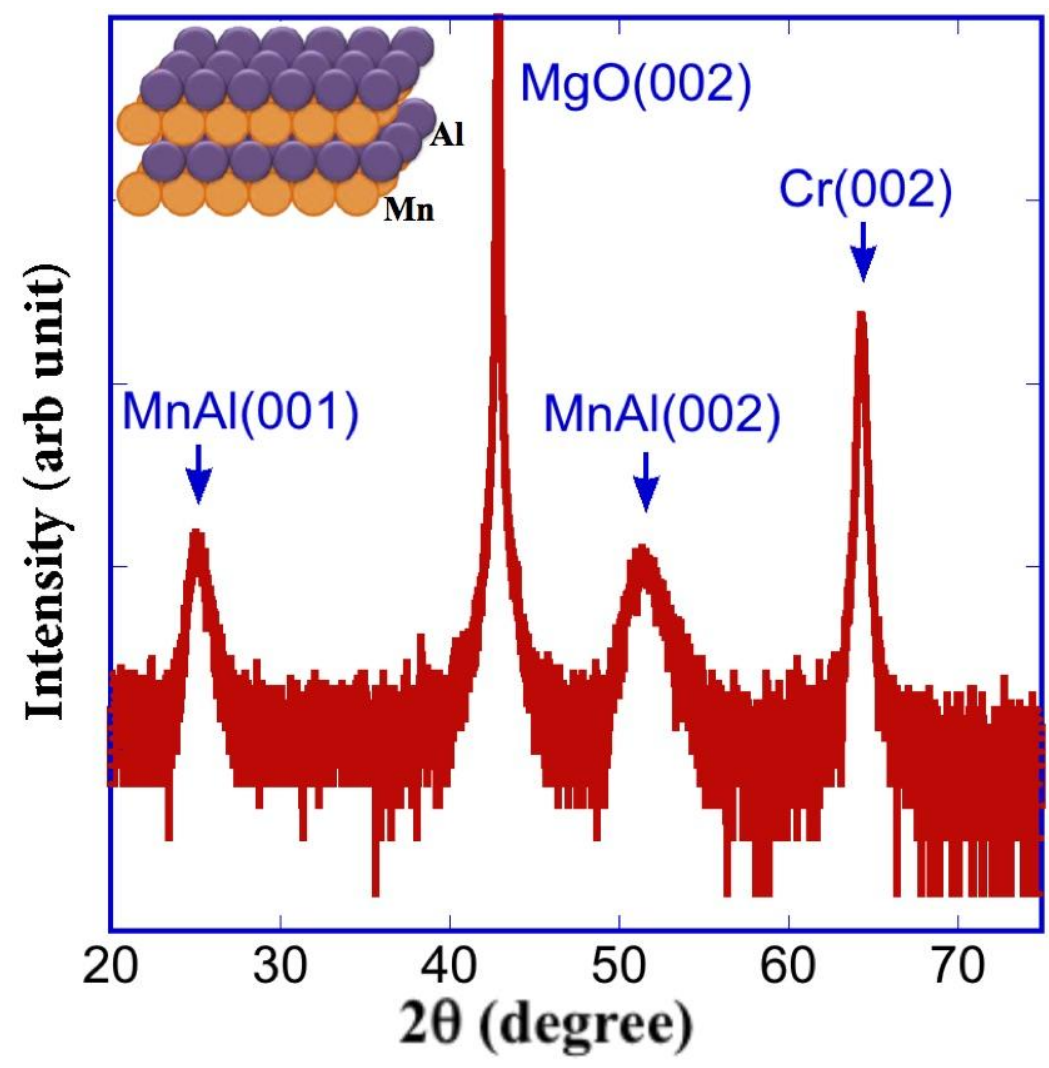

Figure 5.1 2ө XRD scan of MnAl deposited on Cr-buffered $\mathrm{MgO}$ substrate showing the fundamental (002) and superlattice (001) peaks. 


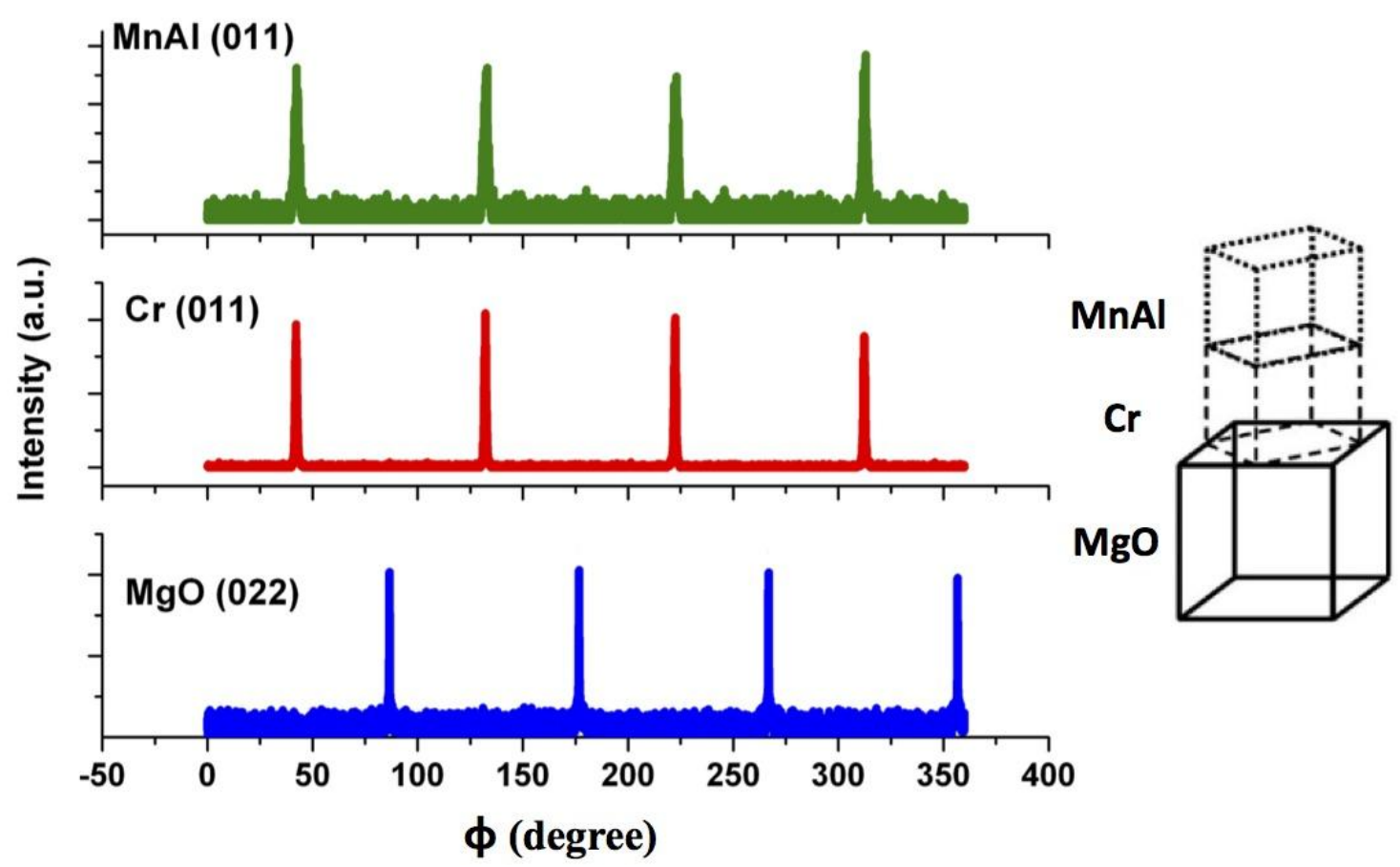

Figure 5.2 $\Phi$ scans on $\mathrm{L}_{0} \mathrm{MnAl}$ deposited on a Cr-buffered $\mathrm{MgO}$ substrate at a growth temperature $200{ }^{\circ} \mathrm{C}$. The green line represents data taken at $2 \theta$ of $41.12^{\circ}(\mathrm{MnAl}(011)$ peak), the red one for data taken at $44.17^{\circ}$ ( $\mathrm{Cr}(011)$ peak) and the blue one for data taken at $62.27^{\circ}(\mathrm{MgO}(022)$ peak). The right side is the schematic diagram of the epitaxial relationships at $\mathrm{MgO} / \mathrm{Cr}$ and $\mathrm{Cr} / \mathrm{MnAl}$ interfaces.

The room temperature $\mathrm{M}-\mathrm{H}$ loops (Figure 5.3) showed a clear indication of perpendicular magnetic anisotropy (PMA). The saturation magnetization $\left(M_{S}\right)$ was calculated to be $462.5 \mathrm{emu} / \mathrm{cm}^{3}$, and the magnetic anisotropy energy $\left(K_{U}\right)$ was $\sim 5.3 \times 10^{6}$ $\mathrm{erg} / \mathrm{cm}^{3}$. Both values were comparable to those of the bulk [83]. It is worth mentioning that 
these values were considerably higher than the corresponding values for MnAl films directly deposited on an $\mathrm{MgO}$ substrate [135]. This is because the $\mathrm{Cr}$ seed layer has a much smaller lattice mismatch with $\mathrm{MnAl}$ as compared to $\mathrm{MgO}$ (6.7\% for $\mathrm{MnAl}$ on $\mathrm{MgO}$ ) [136], which lead to a larger tetragonality in $\mathrm{MnAl}$ with a c/a 1.27, while the c/a is $\sim 1.0$ for $\mathrm{MnAl}$ that is grown directly on the $\mathrm{MgO}$ substrate. As predicted [93, 137], the c/a of 1.27 resulted in a higher magnetic moment.

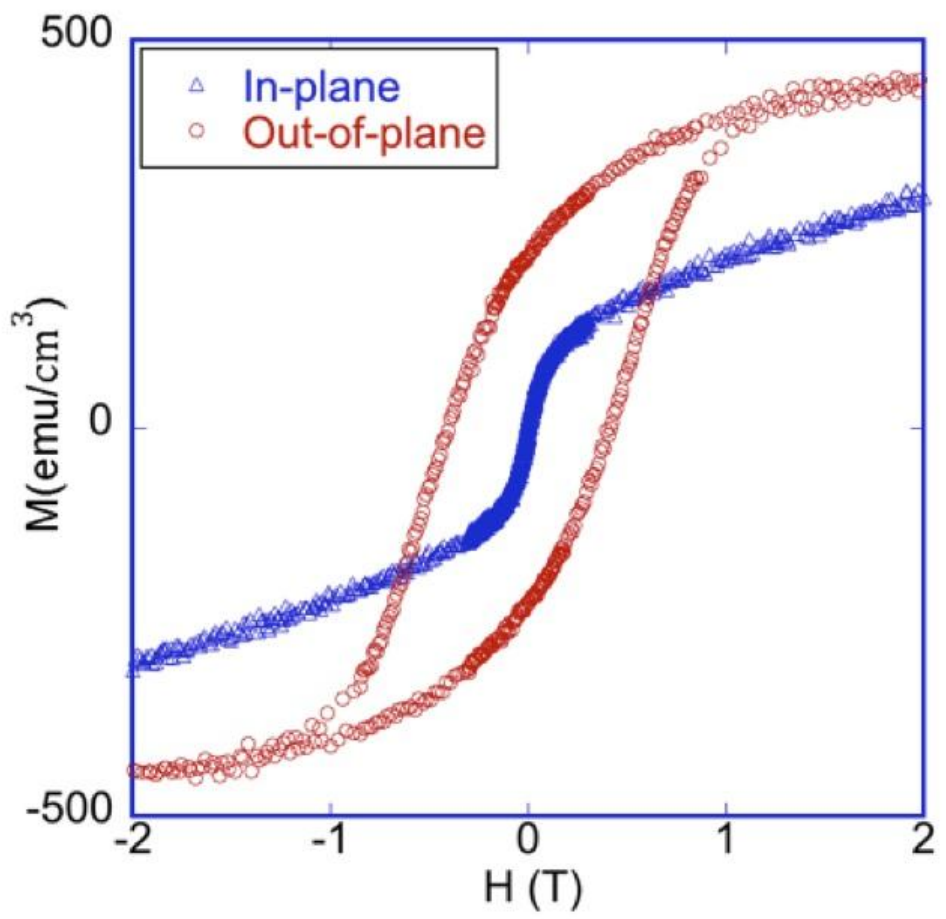

Figure 5.3 In-plane (Blue triangles) and out-of-plane (Red dots) hysteresis loops for MnAl films measured at room temperature. 


\subsubsection{Magnetic domain structure of epitaxy MnAl}

The magnetic domains of the MnAl films were characterized by MFM as shown in Figure 5.4(b). Maze-like stripe domains were observed in the MFM phase image, which was expected for magnetic thin films with strong PMA. The magnetic domain structure is strongly dependent on the ratio of anisotropy to magneto-static energy: $Q=K_{U} / 2 \pi M_{S}^{2}$, where $K_{U}$ is the uniaxial anisotropy constant, $M_{S}$ the saturation magnetization [92]. The calculated $Q$ was $\sim 4$ based on the values of $K_{U}$ and $M_{S}$ from the VSM results at room temperature. For $Q>1$, stripe domains that intersect the surface with PMA are energetically favored $[92,138]$. This can be understood in terms of the balance between anisotropy energy and the demagnetizing energy. The demagnetizing energy prefers an in-plane magnetization for thin films due to the relatively small dipolar interaction at that direction (see discussion in Session 2.5.2), while the perpendicular anisotropy tends to bring the magnetization out-of-plane. Once the PMA become dominant in the system, the stripe domain is formed with an averaged domain size $\sim 230 \mathrm{~nm}$. 

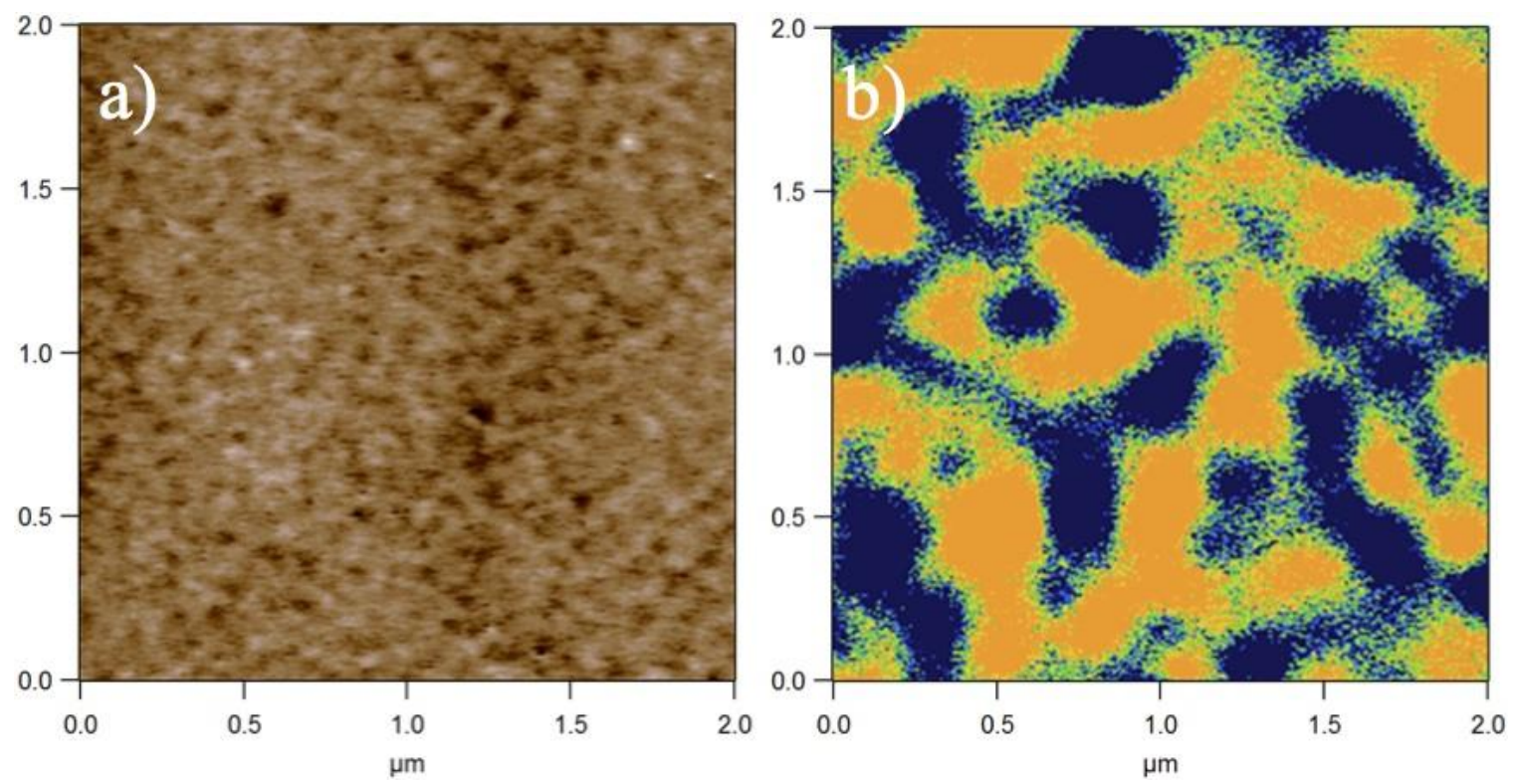

Figure $5.42 \mu \mathrm{m} \times 2 \mu \mathrm{m}$ (a) AFM topography image for the MnAl films and (b) the corresponding MFM image (or phase image) after demagnetized at $30 \mathrm{kOe}$ along the perpendicular direction. The surface roughness RMS is around $2 \AA$.

\subsection{Magneto-transport and domain wall scattering in epitaxial}

\section{$\mathrm{L1}_{0}$ MnAl thin film}

\subsubsection{Current-in-plane patterning}

In order to study the magneto-transport properties of MnAl films, the samples were patterned into a standard Hall bar geometry (as shown in Figure 5.5 inset) using a two-step

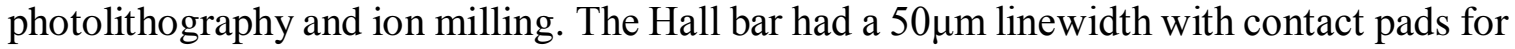
measurement of both longitudinal and transverse (Hall) resistance. Longitudinal 
magneto-resistance $\left(R_{x x}\right)$ measurements were performed on the Hall bar in the temperature range of $50 \mathrm{~K}$ to $320 \mathrm{~K}$ and in magnetic field up to $30 \mathrm{kOe}$, with the field applied: (1) in the film plane and parallel to the electrical current [longitudinal configuration], and (2) perpendicular to the film plane [perpendicular configuration]. The difference of spatial geometry was achieved by manually rotating the sample with respect to the field direction. Low bias currents (0.1-2.0mA) were employed for magneto-transport measurement in order to minimize the thermal effect.

\subsubsection{Magneto-transport in $\mathrm{L1}_{0} \mathrm{MnAl}$}

Figure 5.5 shows the isothermal magnetoresistance ratio (MR) (defined as $\Delta R / R=[R(H, T)-R(0, T)] / R(0, T))$ versus magnetic field curves, which were measured from 50 $\mathrm{K}$ to $320 \mathrm{~K}$ with the field applied out of plane. Above $175 \mathrm{~K}$, the sign of the MR was negative, and an evident two-peak shape was observed. A sign change in MR occurred near $175 \mathrm{~K}$, at which temperature the co-existence of both a negative and positive MR was apparent. Below $175 \mathrm{~K}$, The sign of MR was positive and increased monotonically when the temperature was reduced. 


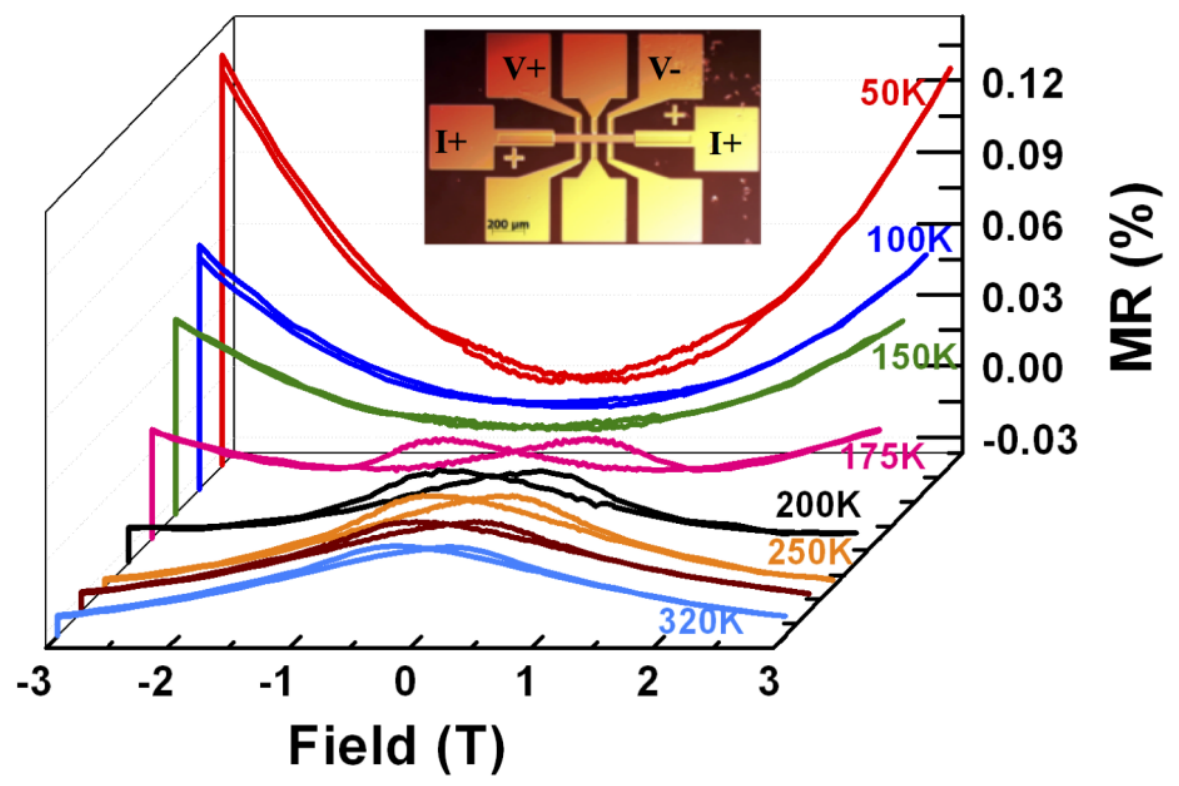

Figure 5.5 MR curves on a $50 \mu \mathrm{m}$ wide Hall bar measured at temperature from $50 \mathrm{~K}$ to $320 \mathrm{~K}$. The external field was applied out of plane. The inset shows the Hall bar pattern with the longitudinal measurement configuration.

The Hall resistivity $\left(\rho_{x y}\right)$ of the MnAl Hall bar was measured from $175 \mathrm{~K}$ to $320 \mathrm{~K}$. The inset in Figure 5.6(a) shows the Hall resistivity $\left(\rho_{x y}\right)$ as a function of field at $300 \mathrm{~K}$. The Hall resistivity, according to Pugh's equation[139], is given by:

$$
\rho_{x y}=R_{0} H_{z}+R_{s} M_{z}
$$

where $H_{z}$ is the perpendicular field, $M_{z}$ is the magnetization, $R_{0}$ and $R_{s}$ are the ordinary and anomalous Hall coefficients. The first linear component comes from the normal Hall effect, while the second extraordinary component is ascribed to the anomalous Hall Effect (AHE). The Hall resistivity followed the hysteretic behavior of the magnetization[140], from which the coercivity $\left(H_{c}\right)$ was extracted at a given temperature. 

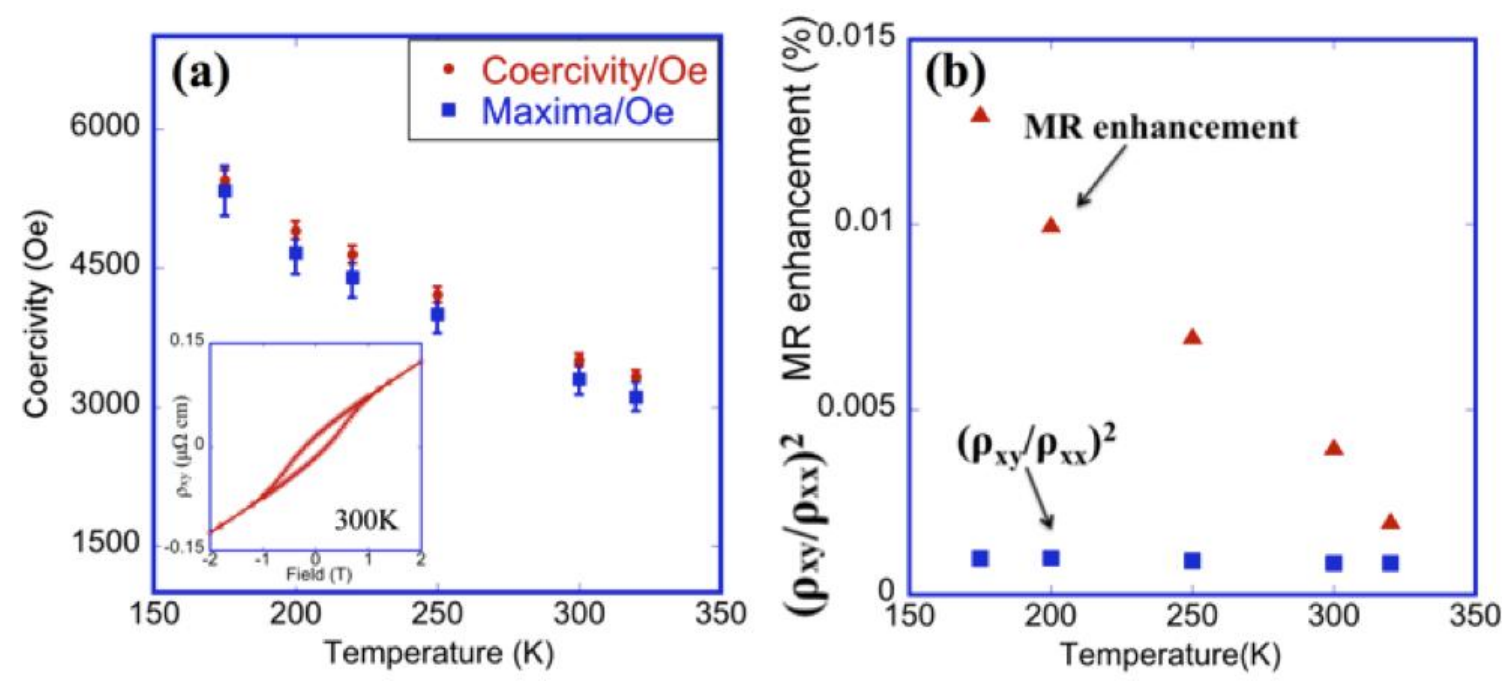

Figure 5.6 (a) Coercivity (Red dots) versus the MR peak positions (Blue squares) for temperature range $175 \mathrm{~K}$ to $320 \mathrm{~K}$. The inset shows the Hall resistivity versus the perpendicular applied field at $300 \mathrm{~K}$. (b) The MR enhancement at the coercive field and $\left(\rho_{x y} / \rho_{x x}\right)^{2}$ vs. temperature.

From $175 \mathrm{~K}$ to $320 \mathrm{~K}$, the peak positions were extracted and compared with the $H_{C}$ obtained from the AHE loop as shown in Figure 5.6(a). The MR reached a maximum when the magnetization was close to zero at $\pm H_{C}$. This implied the existence of magnetic inhomogeneity [141]. In the MnAl films, the inhomogeneity was a consequence of the magnetic domain walls that cause strong spin-dependent scattering on the nanoscale. At the coercive field, more magnetic domains were present thanks to the field-induced domain wall motion and/or domain rotation during the magnetization reversal $[142,143]$. The increase in the domain density led to a substantial increase in the domain wall induced 
scattering of charge carriers, resulting in the enhancement of the resistance.

Extrinsic mechanisms such as anisotropic magnetoresistance (AMR), which contributed to the MR as reported previously [144], did not have a significant effect on the MR in MnAl, because the angle between the magnetization and current was kept unchanged during the sweeping of the external magnetic field.

Berger also proposed that the Hall effect led to a zigzag pathway for the electric current going through stripe domains, and a concomitant increase in the resistivity by $\left(\rho_{x y} / \rho_{x x}\right)^{2}[145]$. In the case of MnAl thin film, $\left(\rho_{x y} / \rho_{x x}\right)^{2}$ was calculated based on the Hall measurements. The results were then compared with the MR enhancement at the coercive field from $175 \mathrm{~K}$ to $320 \mathrm{~K}$ as shown in shown in Figure 5.6(b). The MR enhancement (defined as $\left[R\left(H_{C}\right)-R(0)\right] / R(0)$ ) at coercivity of $0.013 \%$ was one order of magnitude larger than $\left(\rho_{x y} / \rho_{x x}\right)^{2}(\sim 0.001 \%)$ at $175 \mathrm{~K}$. Thus, the Hall effect contribution was of insufficient magnitude to explain the MR enhancement observed here.

Below 150K, the MR became positive, and was quadratic with respect to the external magnetic field, which can be attributed to the Lorentz force on the trajectories of the electrons. The Lorentz force leads to an orbital motion for electrons in an out-of-plane magnetic field, and the Lorentz MR is predicted to increase quadratically as a function of the B field [146, 147].

The MR measured with the field direction parallel to the current direction at $50 \mathrm{~K}$ and $250 \mathrm{~K}$ are shown in Figure 5.7. The sign of the MR was negative for both temperatures and 
no quadratic relation was observed. Here the Lorentz effect was minimized due to the parallel alignment between the electric current and magnetic field. This confirms the fact that the positive MR at low temperatures was caused by the extrinsic contribution from the Lorentz effect. At $50 \mathrm{~K}$ with high magnetic field, the MR showed an upturn to become positive, which may be due to the small misalignment $\left(<5^{\circ}\right)$ of the hall bar with the field direction. It is noted that the Lorentz MR can be suppressed due to the decrease of mean scattering time when the temperature increases [147], which may explain the sign change in the MR at around $175 \mathrm{~K}$.

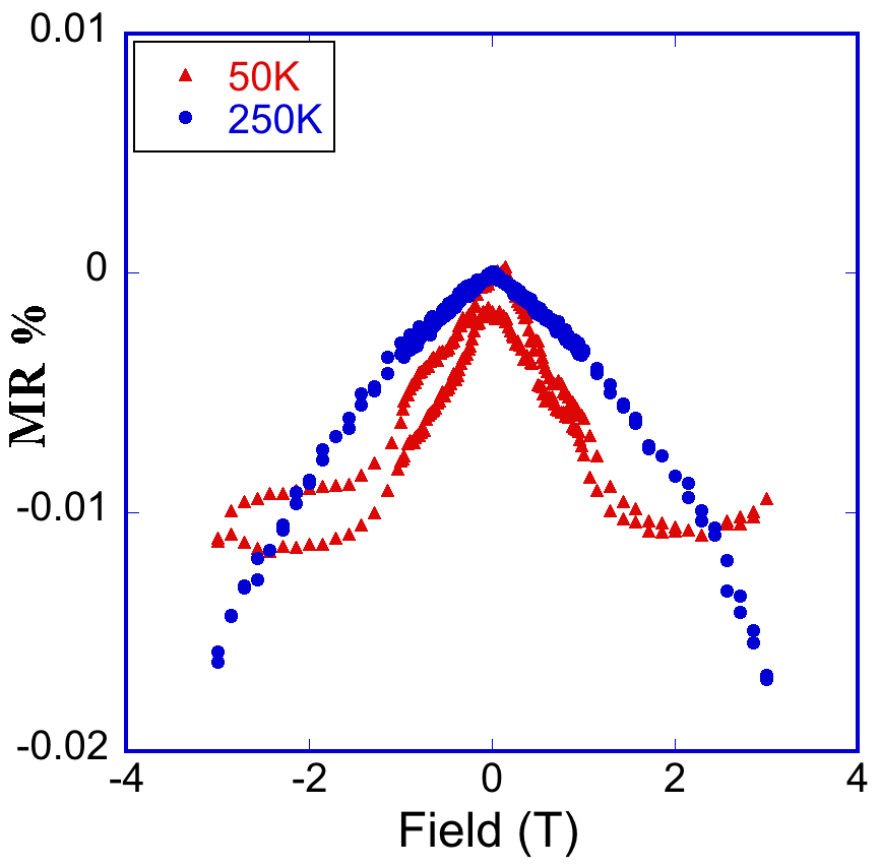

Figure 5.7 MR curves measured at temperature $250 \mathrm{~K}$ (Blue dots) and $50 \mathrm{~K}$ (Red triangles). The external field was applied in plane and along the longitudinal direction. 


\subsubsection{Domain wall scattering study in $\mathrm{L} 1_{0} \mathrm{MnAl}$}

Resistivity measurements with the external field applied in the longitudinal direction $\left(\rho_{\text {long }}\right)$ and the perpendicular direction $\left(\rho_{\text {perp }}\right)$ are shown in Figure 5.8(a), where $\rho_{\text {long }}$ is larger than $\rho_{\text {perp. }}$. MFM images of the same film were scanned in an area of $100 \mu \mathrm{m}^{2}$. Figure 5.8(b) and Figure 5.8(c) show the measured MFM images corresponding to the remanent states of longitudinal and perpendicular measurements, which are marked as points A and B in Figure 5.8(a) respectively. The MFM image demonstrated a larger domain size in B, which can be ascribed to the result of the magnetization process [148]. After removing the out-of-plane field, the uniform magnetized domain broke up into multiple domains to minimize the demagnetizing energy. The magnetic moments in B had to overcome the uniaxial anisotropy and rotate toward the opposite direction to form a domain wall. In the case of A, since the magnetic field already aligned the magnetic moments to an in-plane direction, the magnetic moments could either rotate up or down with no preferred orientation, and therefore it was much easier to form magnetic domains in A. By defining the boundary of domains in the MFM images using the software WSxM [149], the number of domains can be counted statistically and the domain density has been calculated, which gives the domain density of $5.57 / \mu \mathrm{m}^{2}$ and $4.67 / \mu \mathrm{m}^{2}$ for case $\mathrm{A}$ and case $\mathrm{B}$, respectively. The in-plane field leads to a higher domain density and thereby a higher density of DWs, which in turn gives rise to the resistivity. 


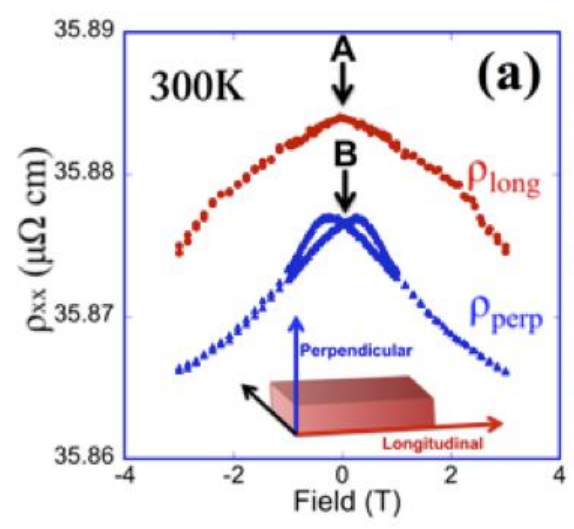

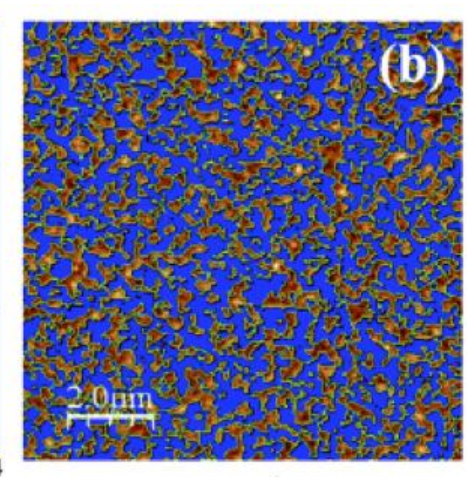

$\mathbf{A}$

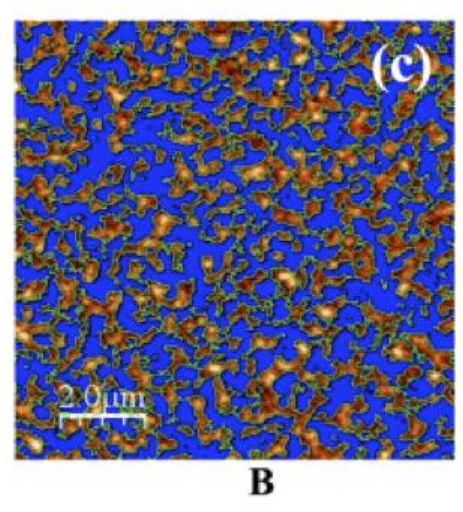

Figure 5.8 (a) The resistivity measured with the field applied in the longitudinal direction (Red dots) and the perpendicular direction (Blue triangles), corresponding to $\rho_{\text {long }}$ and $\rho_{\text {perp }}$ respectively. The remanent states are marked as A and B. (b)\&(c) MFM phase images corresponding to remanent states A and B. The domain boundaries have been highlighted in green color. Density of domains in state A: $5.57 / \mu \mathrm{m}^{2}$ and B: $4.67 / \mu \mathrm{m}^{2}$

In order to extract the DWs scattering contribution to the resistivity quantitatively, the resistivity is expressed by [92]

$$
\begin{aligned}
& \rho_{\text {long }}=\rho_{s}+\frac{\delta_{w}}{d_{\text {long }}} \rho_{D W}+\rho_{A M R / /} \approx \rho_{s}+\frac{\delta_{w}}{d_{\text {long }}} \rho_{D W} \\
& \rho_{\text {perp }}=\rho_{s}+\frac{\delta_{w}}{d_{\text {perp }}} \rho_{D W}+\rho_{A M R \perp} \approx \rho_{s}+\frac{\delta_{w}}{d_{\text {perp }}} \rho_{D W}
\end{aligned}
$$

where $\rho_{\text {long }}$ and $\rho_{\text {perp }}$ are the measured resistivity, $\rho_{s}$ is the resistivity in the saturated state, $\rho_{D W}$ is the extra domain wall induced resistivity, $\rho_{A M R / /}$ and $\rho_{A M R} \perp$ are the AMR contributions corresponding to the cases A and B. At low magnetic field, the MFM images revealed an out-of-plane magnetization for both cases, so the $\rho_{A M R}$ is estimated to be much 
smaller than $\rho_{s}$. In the equation, $d_{\text {long }}$ and $d_{\text {perp }}$ are the average domain sizes, which were estimated from the MFM images to be $200 \mathrm{~nm}$ and $275 \mathrm{~nm}$ for A and B, respectively. Meanwhile, $\delta_{w}$ is the domain wall width. For films with $Q>1$, the domain walls are assumed to be Bloch walls in the center of the film [150]. The wall width is given by equation [148]

$$
\delta_{\text {Bloch }}=\pi \sqrt{A / K_{U}}
$$

where $A$ is the exchange stiffness constant and $K_{U}$ is the uniaxial anisotropy constant $5.3 \times 10^{6} \mathrm{erg} / \mathrm{cm}^{3}$. The exchange stiffness for $\mathrm{L} 1_{0} \mathrm{MnAl}$ has been estimated by [151]

$$
A=3 k_{B} T_{C} / 2 z a
$$

where $k_{B}$ is the Boltzmann constant $\left(1.38 \times 10^{-16} \mathrm{erg} / \mathrm{K}\right), T_{C}$ is the Curie temperature $(690 \mathrm{~K})$ [152], $z=8$ is the number of nearest neighbor atoms, and $a$ is the lattice constant $(2.80 \AA)$. Therefore, $A=6.38 \times 10^{-7} \mathrm{erg} / \mathrm{cm}$. The wall width for MnAl was then calculated to be $\delta_{w}$ $\sim 10.9 \mathrm{~nm}$, which was comparable to the values found in FePt $\left(\delta_{w}=6 \mathrm{~nm}\right)[153]$ and $\mathrm{CoCrPt}$ $\left(\delta_{w}=14 \mathrm{~nm}\right)$ [151]. By combining Eqs. (5-2) and (5-3), and substituting in the above-mentioned information, the equations can be solved in terms of $\rho_{D W} / \rho_{s}$. The DWs scattering contribution to the resistivity is calculated to be $\rho_{D W} / \rho_{s}=1.75 \%$ for the maze states as shown in Figure $5.8(\mathrm{~b}) \&(\mathrm{c})$. This value was smaller than the current-perpendicular-to-wall (CPW) (8.2\%) DWs contribution but larger than the current-in-wall (CIW) (1.3\%) DWs contribution for FePd that had parallel stripe domains [92]. Unlike FePd, MnAl had a maze-like stripe domain that possibly results in an average 
of current conduction channels between CPW and CIW geometry. Based on the averaging of the conductions along the two orientations, the value was in good agreement with the theoretical prediction of Levy and Zhang for typical ferromagnetic materials [87].

\section{$5.4 \mathrm{L1}_{0} \mathrm{MnAl}$ based magnetic multilayers}

\subsubsection{Magnetic hybrid structure}

Pseudo spin-valve structures made of Cr40nm / MnAl20nm / Cr2nm / $\mathrm{Co}_{2} \mathrm{FeAl} 3.5 \mathrm{~nm}$ /Ru5nm were deposited by BTIBD on an $\mathrm{MgO}$ substrate. This is called the magnetic hybrid structure because of the perpendicular magnetic anisotropy and the in-plane anisotropy for $\mathrm{MnAl}$ and $\mathrm{Co}_{2} \mathrm{FeAl}$ respectively.

The in-plane hysteresis loop was measured at different temperatures from $50 \mathrm{~K}$ to 300 $\mathrm{K}$ as shown in Figure 5.9(a). The sharp transition close to the zero magnetic field corresponds to the magnetization reversal of the $\mathrm{Co}_{2} \mathrm{FeAl}$. The magnetization reversal in $\mathrm{Co}_{2} \mathrm{FeAl}$ is mainly accomplished by domain wall moving due to its in-plane anisotropy, while the reversal for $\mathrm{MnAl}$ is mostly achieved by magnetization rotation due to its strong perpendicular anisotropy. The $\mathrm{MnAl}$ layer has a much larger coercive field than $\mathrm{Co}_{2} \mathrm{Fe} \mathrm{Al}$, which agrees with the prediction of the Stoner-Wohlfarth model [154]. The increase of the coercive fields might be related to the enhancement of the PMA for the MnAl layer as the temperature decreased. The same trend was observed for coercive fields in the out-of-plane 
direction, as shown in Figure 5.9(b).
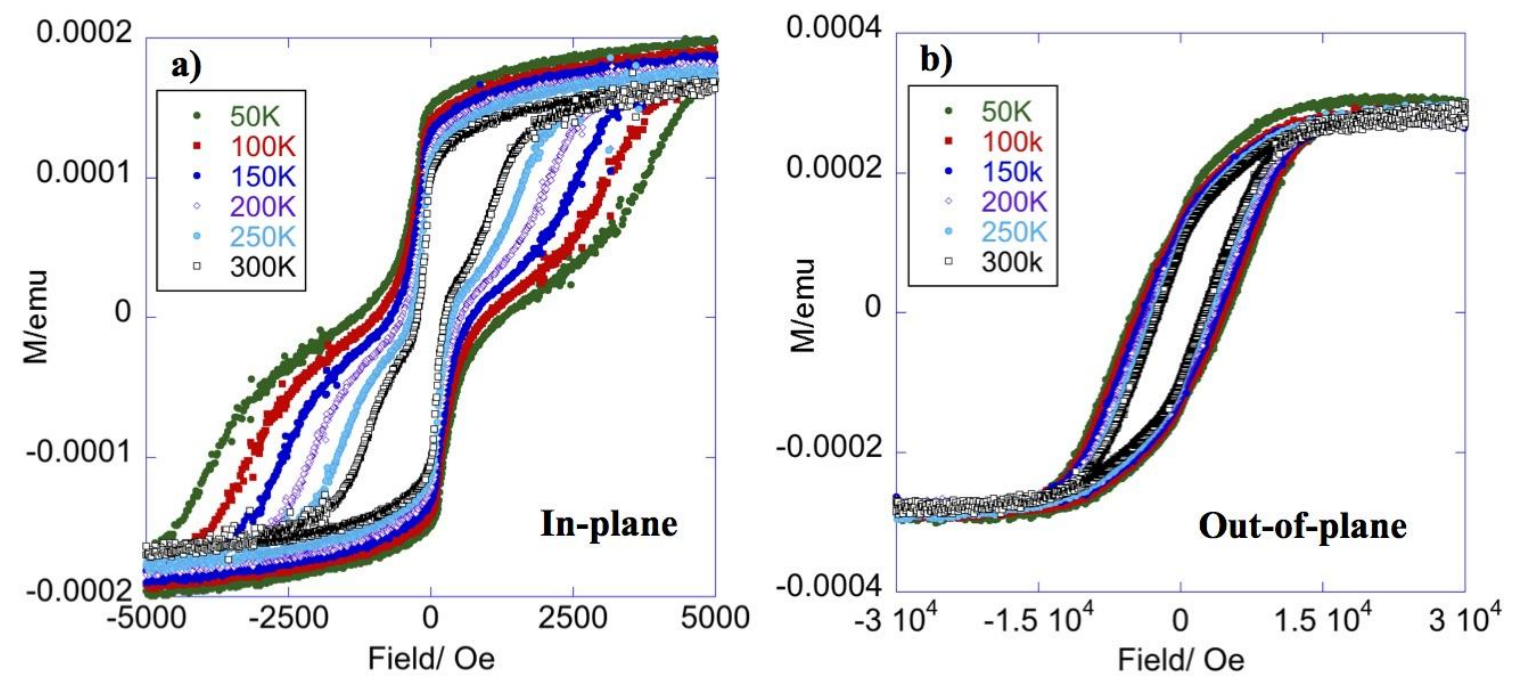

Figure 5.9 In-plane (a) and Out-of-plane (b) hysteresis loops of the magnetic hybrid structure Cr40nm / MnAl20nm / Cr2nm / $\mathrm{Co}_{2} \mathrm{FeAl} 3.5 \mathrm{~nm} / \mathrm{Ru} 5 \mathrm{~nm}$ measured at different temperatures from $300 \mathrm{~K}$ to $50 \mathrm{~K}$.

\subsubsection{Magneto transport in the hybrid structure}

Figure 5.10 shows the in-plane hysteresis loop (a) and the corresponding magneto resistance curve (b) at $150 \mathrm{~K}$. The curves at room temperature shows similar trend. The magnetic states are schematically shown beside the curve in Figure 5.10(b). Again, the relative sharp transition can be attributed to the reversal of the $\mathrm{Co}_{2} \mathrm{FeAl}$ layer, while the gradual change is mainly due to the rotation of the MnAl magnetization. A very small MR ratio of $0.05 \%$ was observed in the pseudo spin-valve at $150 \mathrm{~K}$. It has been reported that TMR was not observed in magnetic tunnel junction made of substrate/Cr(40) $/ \mathrm{MnAl}(5)$ 
$/ \mathrm{MgO}(2) / \mathrm{Co}_{50} \mathrm{Fe}_{50}(5) / \mathrm{Ta}(5)$ (unit in $\mathrm{nm}$ ) due to the lattice mismatch between $\mathrm{MnAl}$ and $\mathrm{MgO}[84]$. However, in our case, the $\mathrm{MnAl} / \mathrm{Cr}$ interface is coherent in lattice constant and is very smooth with RMS $\sim 2 \AA$.

In similar $\mathrm{L}_{0} \mathrm{FePt}$ system, low GMR $\sim 0.56 \%$ was observed in the $\mathrm{FePt} / \mathrm{Cu} / \mathrm{NiFe}$ structure at $300 \mathrm{~K}$ and TMR ratio $\sim 40 \%$ was observed at $4.2 \mathrm{~K}$ on FePt based MTJ structures [155-157]. Experimental result revealed a spin polarization of $\sim 40 \%$ in $\mathrm{L} 1_{0} \mathrm{FePt}$ film with a high chemical ordering constant of $S \sim 0.9$ [158]. Based on the first-principle calculations of spin-polarized density of states (DOS) in MnAl and FePt, the spin polarization of $\mathrm{L} 1_{0} \mathrm{MnAl}$ was estimated to be much smaller than $\mathrm{L} 1_{0} \operatorname{FePt}[93,159,160]$. More experiments using the technique of Point Contact Andreev Reflection spectroscopy (PCAR) need to be carried out to confirm the spin-polarization value in $\mathrm{L} 1_{0} \mathrm{MnAl}$.
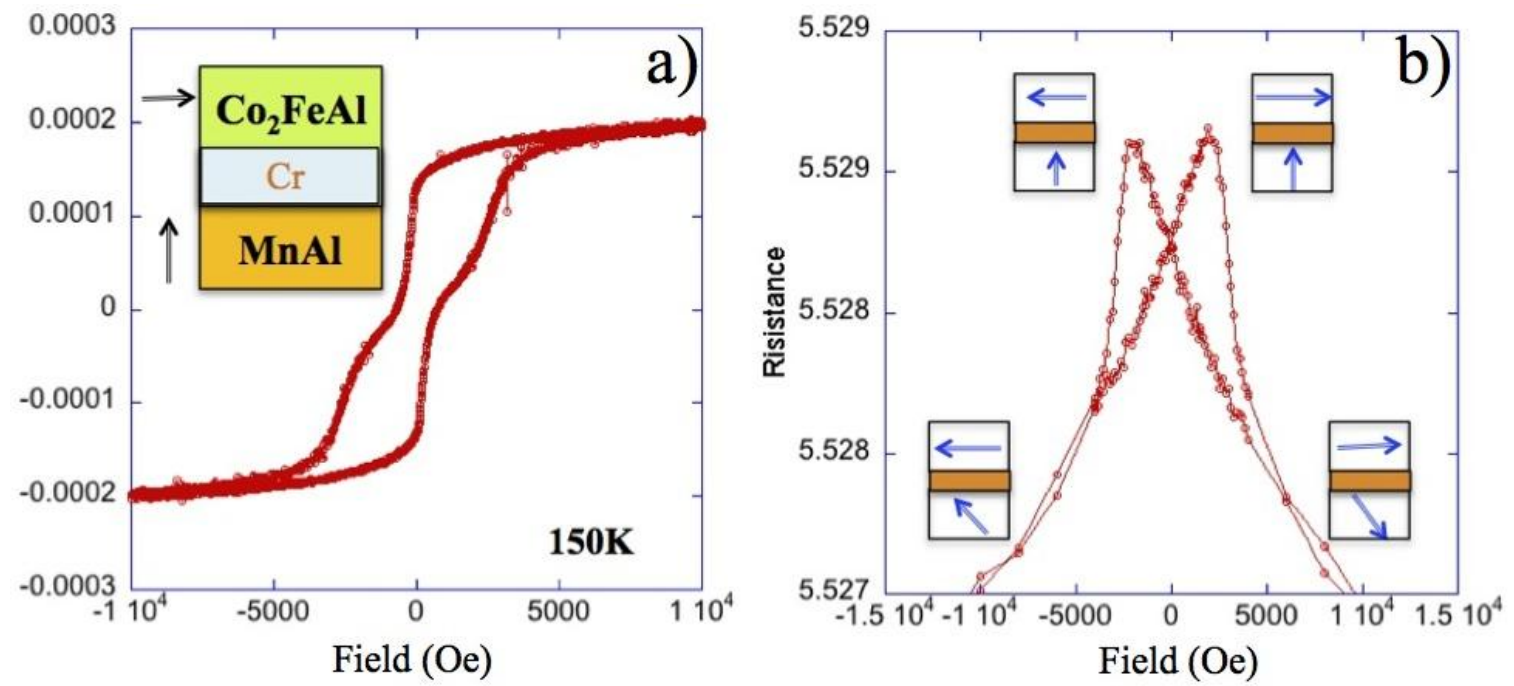

Figure 5.10 (a) M-H loop of the pseudo spin-valve Cr40nm / MnAl20nm / Cr2nm / $\mathrm{Co}_{2} \mathrm{FeAl3} .5 \mathrm{~nm} / \mathrm{Ru} 5 \mathrm{~nm}$ at $150 \mathrm{~K}$. (b) The corresponding MR(in $\Omega$ ) measured at $150 \mathrm{~K}$. 


\subsection{Summary}

In this Chapter, magneto-transport properties of epitaxial $\mathrm{L}_{0} \mathrm{MnAl}$ films with high chemical ordering $\sim 0.89$ and strong PMA $\sim 5.3 \times 10^{6} \mathrm{erg} / \mathrm{cm}^{3}$ have been investigated. A temperature-dependent magneto-resistance change was observed on the Hall bar patterned sample. From $320 \mathrm{~K}$ to $175 \mathrm{~K}$, the low field MR enhancement was linked to the DWs scattering of charge carriers. Further analysis on remanent states' MFM images and the corresponding resistivity demonstrated the contribution of DWs to the electric resistivity of MnAl. A MR sign change occurred around $175 \mathrm{~K}$, and the MR turned positive and quadratic with respect to field when the temperature was below 175K. The longitudinal measurements suggest that the MR from the Lorentz effect became dominant at temperatures below $175 \mathrm{~K}$.

Magnetic hybrid structure and all-perpendicular structure PSVs have been fabricated by taking advantage of the strong PMA in MnAl. Magnetic static and magneto transport properties for both configurations have been studied. Low MR value of $\sim 0.05 \%$ was observed on the hybrid PSV possibly due to the small spin-polarization in the thin MnAl films. Further confirmation can be carried out using the PCAR technique. 


\section{Chapter 6 Current perpendicular to plane (CPP) device fabrication}

\subsection{Introduction}

The fabrication techniques used to make magnetic nanostructures can vary widely depending on the type of magnetic nanostructure being studied. The two common types of patterned magnetic nanostructures are nanostructures in the current-in-plane (CIP) geometry, and nanostructures in the current-perpendicular-to-plane (CPP) geometry. These different geometries are presented in Figure 6.1. In the current-in-plane (CIP) geometry, the current flows along the layers, and the electrodes are located on one side of the structure. The CIP geometry can be achieved through a two-step photolithography process. A typical CIP pattering is the Hall bar structure as shown in Figure 6.1(b). However, the CIP geometry is limited for GMR measurement on metallic magnetic multilayers and cannot be applied to TMR measurement due to the large resistivity of the barrier layer in a magnetic tunnel junction. In the current perpendicular to plane (CPP) geometry, the current carrying leads are coupled to the nanostructure perpendicular to the layers, so that the current through the device runs perpendicular to the plane of the layers. The CPP geometry needs the top and bottom contact electrodes to be defined separately, and therefore requires multi-steps photolithography processing. 
a)

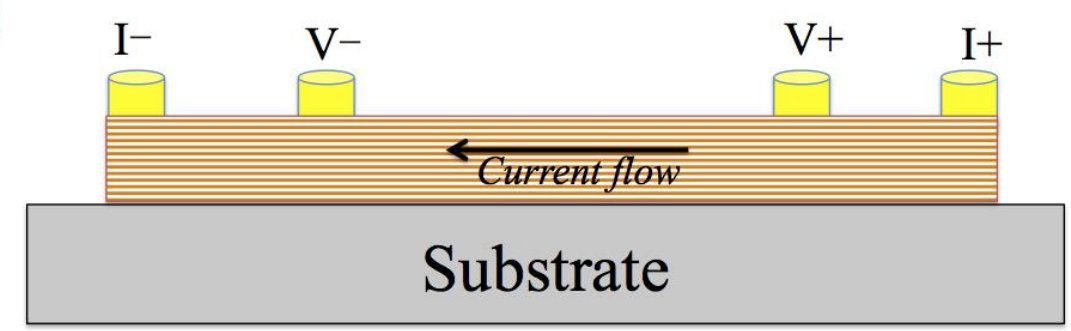

b)

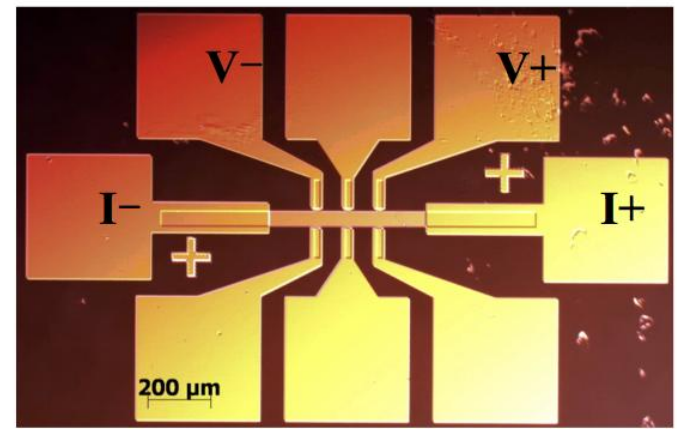

c)

c) $\mathrm{V}+\quad \mathrm{I}+$

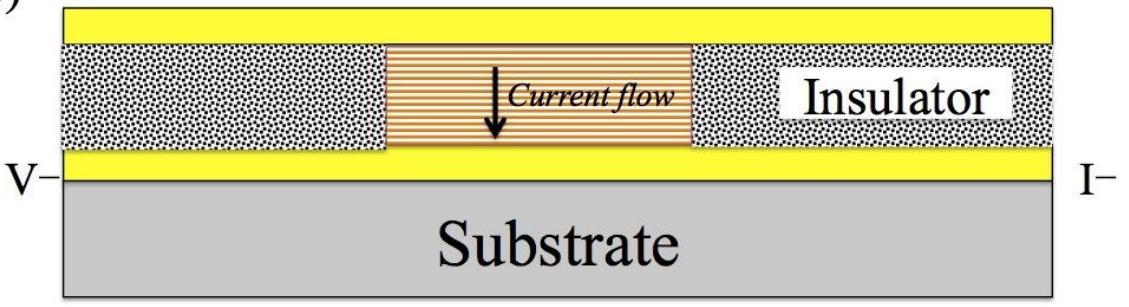

d)

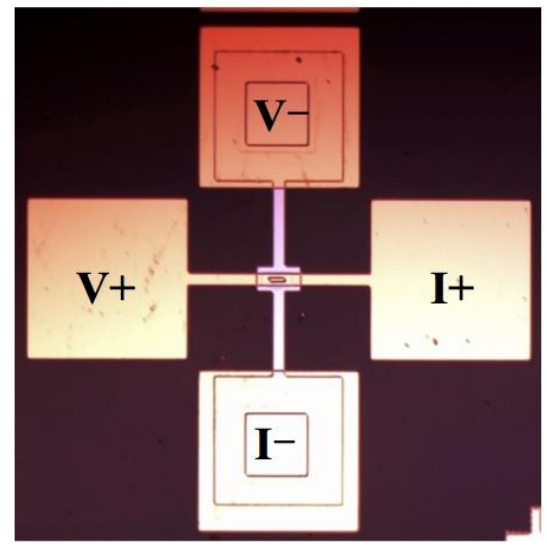

Figure 6.1 (a) Schematic of a CIP patterning structure. (b) A Hall bar structure with CIP geometry. (c) Schematic of a CPP patterning structure. (d) A magnetic structure with CPP geometry. 
Depending on the size of the nanostructure, successfully fabricating CPP devices can be quite difficult, especially as the device dimensions shrink to about equal to the wavelength of the light used [161] ( $\lambda \sim 400 \mathrm{~nm}$ for the Hg lamp source in UVa clean room). This chapter will focus on techniques that I have developed to fabricate $500 \mathrm{~nm}$ CPP spin-torque nano-oscillator structures at the clean room facility.

\subsection{CPP device fabrication}

\subsubsection{Overview of CPP device fabrication}

Current-perpendicular-to-the-plane (CPP) magnetic nanostructures can be fabricated with two general techniques, an additive process and a subtractive process. An additive process is a process where the nanostructure is defined by adding material in order to form the nanostructure, while subtractive process is a process where one first starts with a bulk film and removes material in order to define the nanostructure.

One technique for fabricating CPP devices that uses an additive process is the liftoff process [162]. First a bottom metal electrode is defined and an insulating material is deposited above the electrode. A small diameter hole is etched in this insulating layer. Next, a magnetic multilayer is deposited onto this structure. At the location of the hole the multilayer will deposit in the hole on the bottom electrode. Away from the hole, material is deposited above the insulator. Enough material is deposited so that the hole is filled up, and the bottom electrode makes contact to the continuous multilayer film, through the 
nanostructures. The multilayer film that deposits over the insulating material acts as the top electrode. The critical step in this process is the deposition of the metallic multilayers that make up the nanostructure. For proper functioning of a multilayer device, the individual layers should be uniform and the layers should not be shorted at the edges. However, deposition into a hole will inherently make the layers non-uniform due to shadowing effects and may even result in shorting at the edges of the device. In a word, when the devices of interest become too small, and the edges dominate the function, this fabrication technique ceases to be effective.

At UVa, instead of depositing a pillar, we start with a bulk film and use a subtractive process to leave a pillar above a metal electrode. We then insulate the top of the pillar from the bottom electrode to protect from shorts. The insulation is then selectively removed until only the top of the pillar is uncovered. Finally, a top electrode is added. This leaves our device of interest sandwiched between a top and bottom electrode. In general, the quality of these devices depends primarily on the ability to grow uniform and smooth magnetic layers, and also on the ability of the subtractive process to define the nanostructure without leaving any material on the edge of the device, which can cause shorting. With a subtractive process there is no limitation as to the type of deposition process that can be used to grow the magnetic multilayer, so any suitable high quality deposition technique could be used. Modern deposition techniques can grow multilayer films that are extremely uniform ( $\pm 1 \%$ across a 5 inch wafer) and smooth (roughness $<2 \AA$ ) [163]. Finally, the 
photolithography process, with proper control, can be tailored to leave little or no unwanted material at edge of the device pillar, eliminating shorting.

\subsubsection{0-nm device fabrication process}

The CPP fabrication process starts with the growth of the continuous films that will eventually be patterned into the nanopillar. The films are grown on 2-inch (100) orientation Si wafers, which have been thermally oxidized. Si wafers without a high temperature oxidation process can conduct current, thus artificially altering the measured transport properties of our CPP devices. An X-Ray Reflection (XRR) measurement shows a $200 \mathrm{~nm}$ thick $\mathrm{SiO}_{2}$ is deposited on top of $\mathrm{Si}$, which is sufficient to prevent the conduction of current through the substrate.

Wafers larger than 2 inch could be used, however, no real advantage is gained. This is due to two reasons, uniformity issues and processing time. Certain processing parameters, such as deposition rates or etching rates, can vary across the wafer in the processing systems available to us. Because our ability to fabricate working devices depends heavily on these parameters, their uniformity, or rather non-uniformity, can lead to functional devices in one section of the wafer and nonfunctional devices in another section of the wafer. Add to this that certain UVa clean room facility tools limit the usable area of the wafer to slightly less than the size of a 2-inch wafer, we see that larger size wafers will not result in a gain in the number of functional devices per wafer. In addition, the use of larger 
wafers will slow down fabrication time.

The continuous films are deposited by the BTIBD sputtering system. The details of the multilayer deposition process can be found in Chapter 3. The complete structure of the thin film was $\mathrm{SiO}_{2}$ (substrate)/20 nm Ru/2 nm CoFe/5 nm Cu/6 nm NiFe/5 nm Ru, where the $20 \mathrm{~nm} \mathrm{Ru}$ layer is used to reduce the contact resistance, and the thin $\mathrm{Ru}$ capping layer is added to prevent NiFe from oxidization. It is important to keep the thickness of the bottom electrode relatively thick such that the resistance of the leads is smaller than the actual patterned device. This reduces the effect of spreading resistance in the leads [164], an effect due to the current exiting the nanostructure and spreading as it enters the electrode, which can lead to a dominance of the electrical transport properties by the leads themselves rather than the device. Since this spreading resistance term will not be magnetic field dependent, this effect will artificially reduce our GMR ratio. However, care must be taken as to not use too thick a bottom electrode, as this will increase the roughness of the films, and therefore affect the interlayer coupling between the fixed layer and free layer. For the $20 \mathrm{~nm}$ thick bottom Ru electrode we find the roughness RMS is around $2 \AA$ while a $50 \mathrm{~nm}$ thick Ru layer yields a roughness RMS of $5 \AA$. 


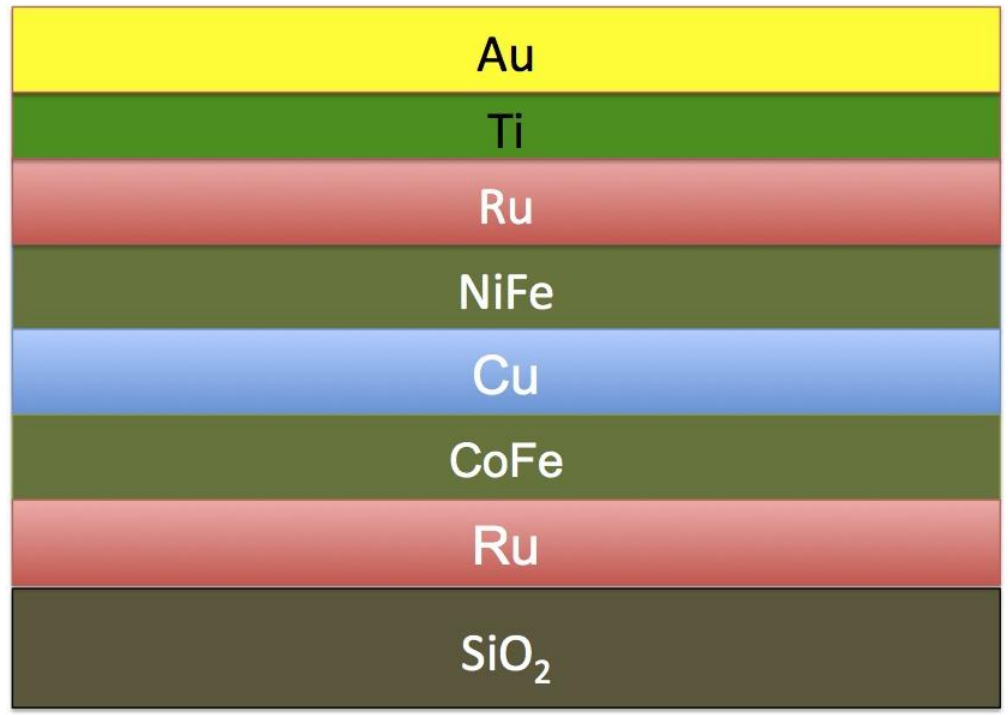

Figure 6.2 The multilayer structure deposited by BTIBD, the Au/Ti layer is deposited afterward.

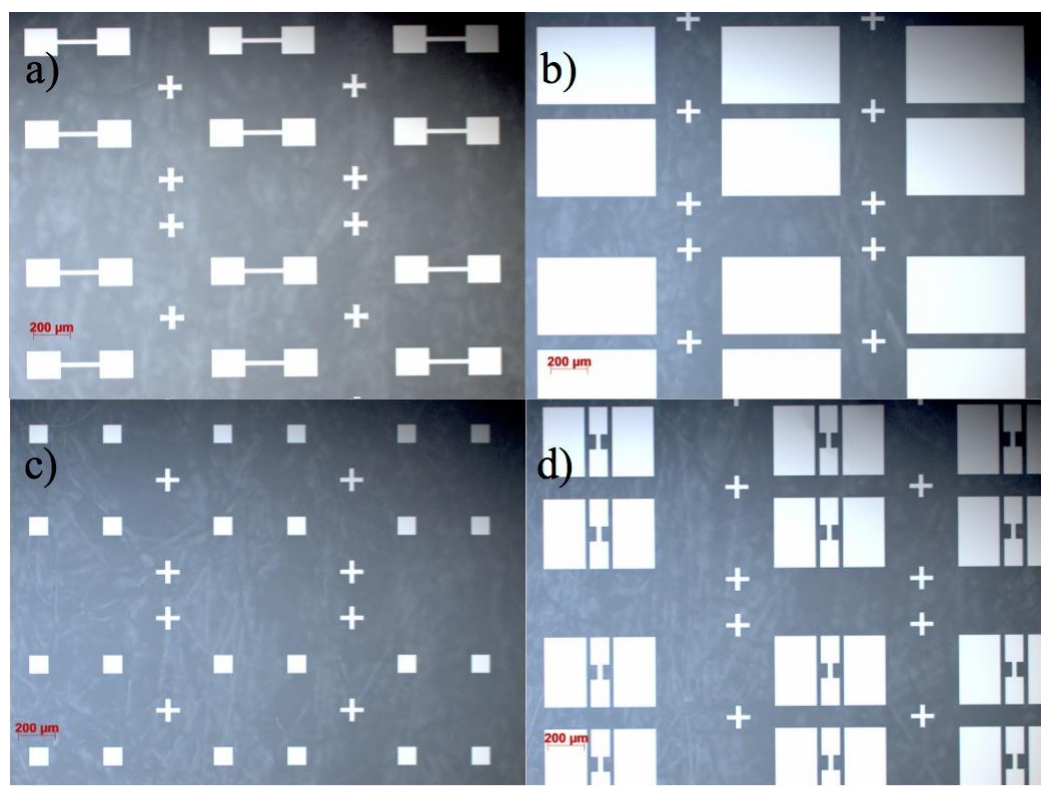

Figure 6.3 Photolithography masks. (a) Layer 1 is to define the bottom electrodes. (b) STNO pillars are defined by layer 2. (c) Layer 3 is to open the contact area in the passivation layer. (d) Layer 4, to define the top contact pads.

Before the patterning process, the thin film is capped with Ti $2 \mathrm{~nm} / \mathrm{Au} 15 \mathrm{~nm}$ by an 
Electron Beam Directed Vapor Deposition (EB-DVD) process. The multilayer structure is illustrated in Figure 6.2. The Titanium(Ti) layer is inserted to increase the adhesion of $\mathrm{Au}$ to the $\mathrm{Ru}$ surface. Au is used as the top layer of the capping stack because of its low reactivity. It does not form a native oxide layer, so it is relatively easy to make electrical contact to this material. Most importantly, Au does not react with photo, electron beam resist or even the $\mathrm{O}_{2}$ plasma, which is important when patterning the film.

\section{Defining the bottom electrode}

The first step in the patterning process is to define the bottom electrode and leads. This is done with photolithography and ion milling. The normal process for photolithography starts by cleaning the wafers with the standard technique (spin with Isopropyl alcohol, Ethanol, Methanol) then $110{ }^{\circ} \mathrm{C}$ hot plate baking for 5 minutes. The hexamethyldisilzane (HMDS) is spin-coated on the wafer surface to promote the adhesion of photoresist. Next AZ 5214 photoresist is applied by spinning at 4000 RPM for 30 seconds. After spinning I bake the wafer on a $100{ }^{\circ} \mathrm{C}$ hotplate for 90 seconds. Once the resist is spun on the wafer, the MJB4 (Suss Microtec Inc.) is used to expose the desired pattern on the wafer with an exposure light density of $75 \mathrm{~mJ} / \mathrm{cm}^{2}$ and an exposure time of 15 seconds. The MJB4 projects the pattern from a mask onto a wafer. The first layer mask is shown in Figure 6.3(a). After the exposure the resist is developed in AZ 400K developer (1:4 with DI water) for 50 second with slight agitation. The wafer is then post-baked on $110{ }^{\circ} \mathrm{C}$ hot plate for 5 
minutes, which is necessary in order to harden the photoresist and improve adhesion of the photoresist to the wafer surface. Then, the wafer is transferred into a beaker of DI water. Finally the wafer is removed, sprayed with DI water, and blown dry.

This photolithography recipe has a disadvantage that it tends to leave a thin layer of photoresist residue on the wafer, even after development. This layer of residue can interfere with following fabrication steps, so it must be removed. This is done by briefly exposing the wafer to $\mathrm{O}_{2}$ plasma, which etches the residual resist. The Au capping layer effectively protects the underneath multilayer structure since $\mathrm{Ru}$ tend to react with $\mathrm{O}_{2}$ at high temperature. For this step we use the MARCH $\mathrm{O}_{2}$ plasma system (Nordson Inc.) with $\mathrm{O}_{2}$ flow rate of 40 SCCM, 100 mTorr, 100 Watts for 15 seconds.

In order to define the leads, the exposed metal needs to be removed. For this, I use the ion milling system in UVa clean room (Oxford Instruments Plasmalab System 100). In ion milling, high energy (>150 eV) $\mathrm{Ar}^{+}$ions are directed toward a substrate, where they the sputter material away. It is important to know that different materials will have different ion mill rates. This depends on the size of the atom and its nearest neighbor binding energy. Au, with a low nearest neighbor binding energy and large atomic mass, has a high ion-milling rate.

In this ion-milling step we mill through the entire multilayer stack, down to the $\mathrm{SiO}_{2}$ layer. To prevent excessive overheating of the wafer during the etching process, L-grease has been applied to the backside of the wafer to aid the heat transfer. A platter with Helium 
flowing on the back is used for mounting the wafer to facilitate the heat transfer from the wafer to the environment. Besides, the wafer has been placed in a relative low-temperature chamber cooled down by liquid nitrogen. I tested the etching under a series of temperature ranging from $0{ }^{\circ} \mathrm{C}$ to $50{ }^{\circ} \mathrm{C}$, and $10^{\circ} \mathrm{C}$ turned out to be adequate to keep the photoresist from overheating. For our typical film thicknesses, this corresponds to a mill time of $\sim 480$ seconds. It is important that the initial milling is not done in one long run, instead the mill time must be broken up into 60 seconds intervals. Again, this is to prevent excessive overheating of the photoresist by the incident ion beam, which can make its removal extremely difficult.

Photoresist that has been heated during ion milling does not readily dissolve in acetone, and as a result a somewhat elaborate resist strip process has been developed. First the wafer is soaked in acetone for 30 minutes. This will cause the damaged photoresist to start flaking. Then the wafer is agitated to remove these flakes. This soak and agitation process is repeated several times, each time using a clean acetone beaker. When there are no more visible flakes the wafer is placed in an acetone beaker that is placed in a low-power ultrasound bath for around 5 minutes. The wafer is then removed, sprayed with IPA, and blown dry. A MARCH $\mathrm{O}_{2}$ plasma process will also be applied to further remove the photoresist residue. Figure 6.4(a) shows a schematic cross section of the device and Figure 6.4(b) shows the corresponding SEM image with bonding pads and leads after the resist has been removed. 


\section{Defining the nanostructure}

In this step a $500 \mathrm{~nm}$ pillar is defined only by using a photolithography process. First I use our standard photolithography recipe to clean and bake the wafer passed on from the previous step. Next I apply a negative photoresist AZ nLOF 2020 and spin at 6000 RPM for 30 seconds. After spinning, the wafer is pre-baked on a $110^{\circ} \mathrm{C}$ hotplate for 60 seconds. Since the minimum feature on the mask is $500 \mathrm{~nm}$ and this feature has to be defined exactly in the center of the bottom layer, an automatic alignment system need to be used to precisely align the cross-mark and control the exposure process. This is done on the EVG 600 photolithography system. The exposure light density is $85 \mathrm{~mJ} / \mathrm{cm}^{2}$. After the exposure, the wafer is post-baked on a $110{ }^{\circ} \mathrm{C}$ hotplate for 60 seconds and then is developed in 300-MIF developer for 30 seconds with slight agitation. Finally the wafer is removed, sprayed and cleaned with DI water, and blown dry. The thickness profile measured with a profilometer reveals a photoresist layer around $1.5 \mu \mathrm{m}$.

The wafer is then dry etched with $\mathrm{Ar}^{+}$using the same recipe as the first layer. It is important that the CoFe layer is only partially patterned so that it is relatively insensitive to the spin transfer torques due to its large volume and its coupling to the extended film. To achieve this purpose, I tested a series of etching times varying from 60 s to 500 s on small pieces of test wafers. Then the etched step is measured by atomic force microscopy (AFM). Figure 6.5 shows a typical step with a 300s etching time. The average etching rate on our multilayer structure at $10{ }^{\circ} \mathrm{C}$ is calculated to be $\sim 0.05 \mathrm{~nm} / \mathrm{s}$. 

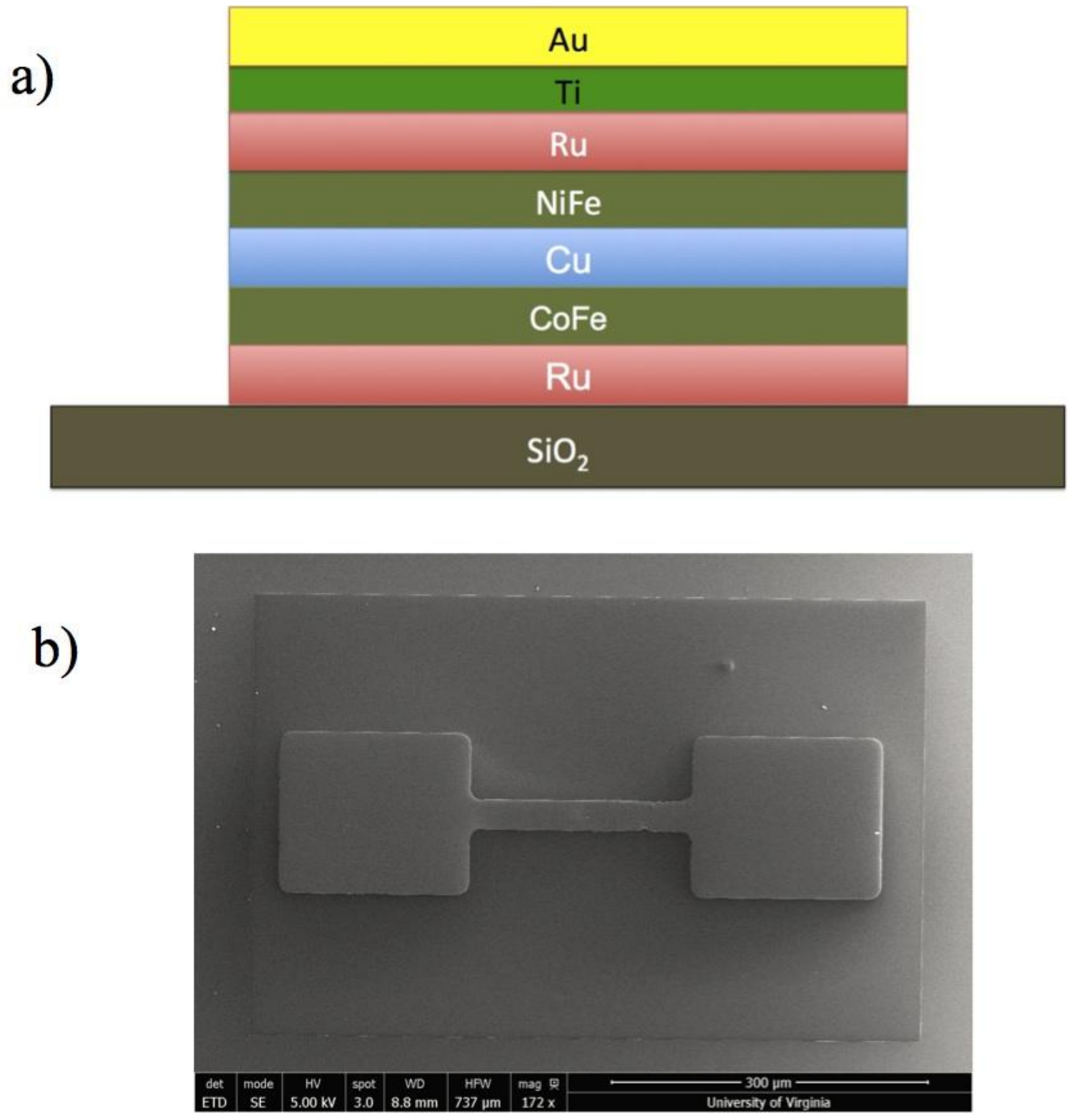

Figure 6.4 (a) A schematic showing the cross section of the bottom electrode. (b) A SEM image of the bottom electrode. 

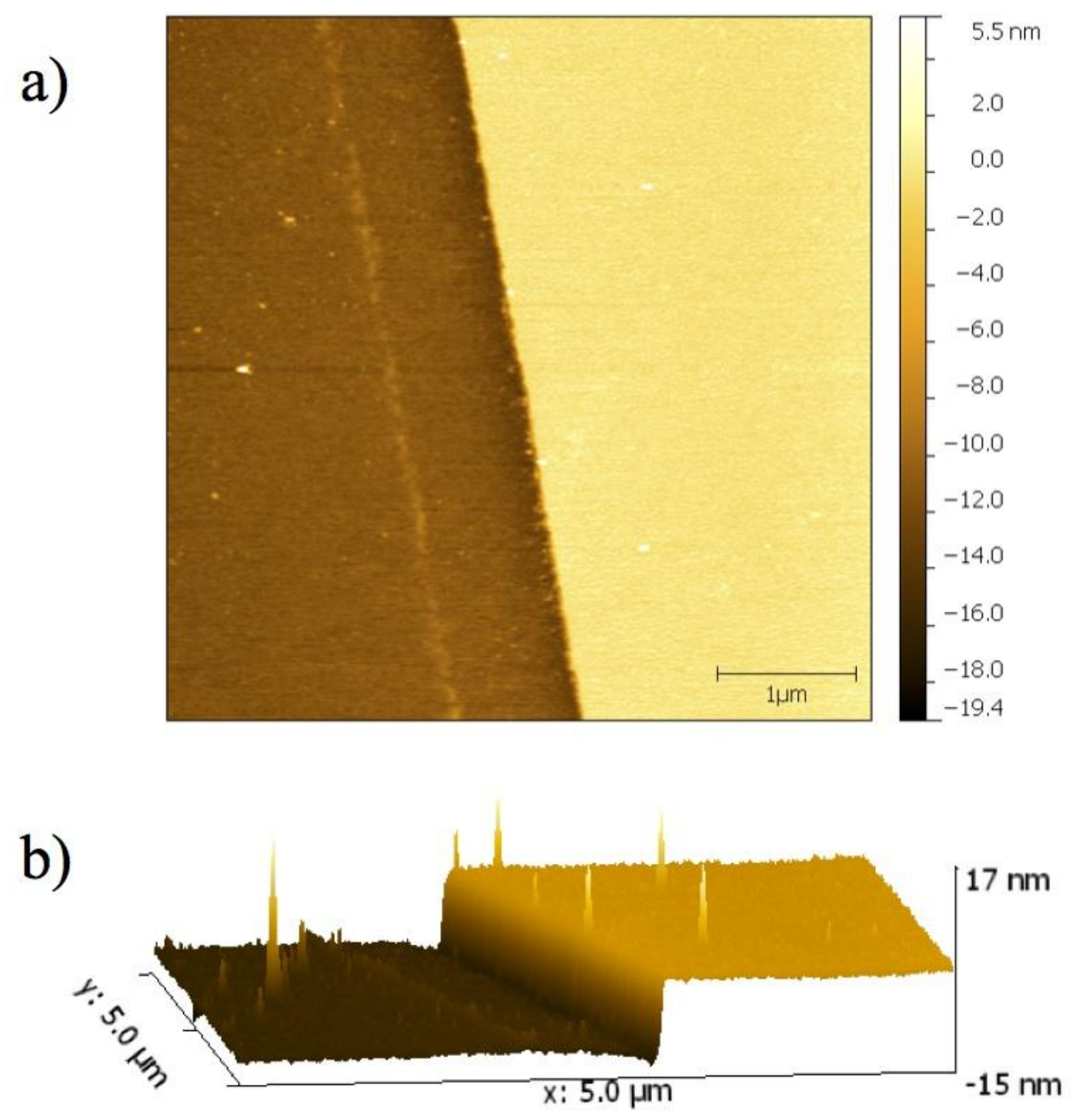

Figure 6.5 (a) A $5 \mu \mathrm{m} \times 5 \mu \mathrm{m}$ AFM scan on the edge of an etched step. (b) A 3D view of the step with scale showing the etching depth of $12.53 \mathrm{~nm}$. 
Since the etching depth might not strictly follow the linear relationship with the etching time, I developed an alternative way to monitor the ion milling by looking at the change of hysteresis loop utilizing vibrating sample magnetometer (VSM). Figure 6.6(a) shows the hysteresis loop of a pseudo spin valve structure with two ferromagnetic layers. The larger coercive field corresponds to $\mathrm{CoFe}$, while the smaller one corresponds to $\mathrm{NiFe}$. Based on the HLoop, $M_{\mathrm{NiFe}}-M_{\mathrm{CoFe}}=4.67 \times 10^{-5} \mathrm{emu}$, and $M_{\mathrm{NiFe}}+M_{\mathrm{CoFe}}=1.86 \times 10^{-4} \mathrm{emu}$, a simple calculation concludes that: $M_{\mathrm{NiFe}}=1.16 \times 10^{-4} \mathrm{emu}$ and $M_{\mathrm{CoFe}}=7.0 \times 10^{-5} \mathrm{emu}$. To calibrate the etching time, we etch the above-mentioned PSV test wafer and measured the M-H loop again as shown in Figure 6.6(b). After the etching, the MH loop shows a coercive field corresponding to single CoFe layer. Besides, the total magnetization $\sim 6.32 \times$ $10^{-5}$ after etching is slightly smaller than the initial value for CoFe single layer, which conforms the partial etching for the CoFe layer. 

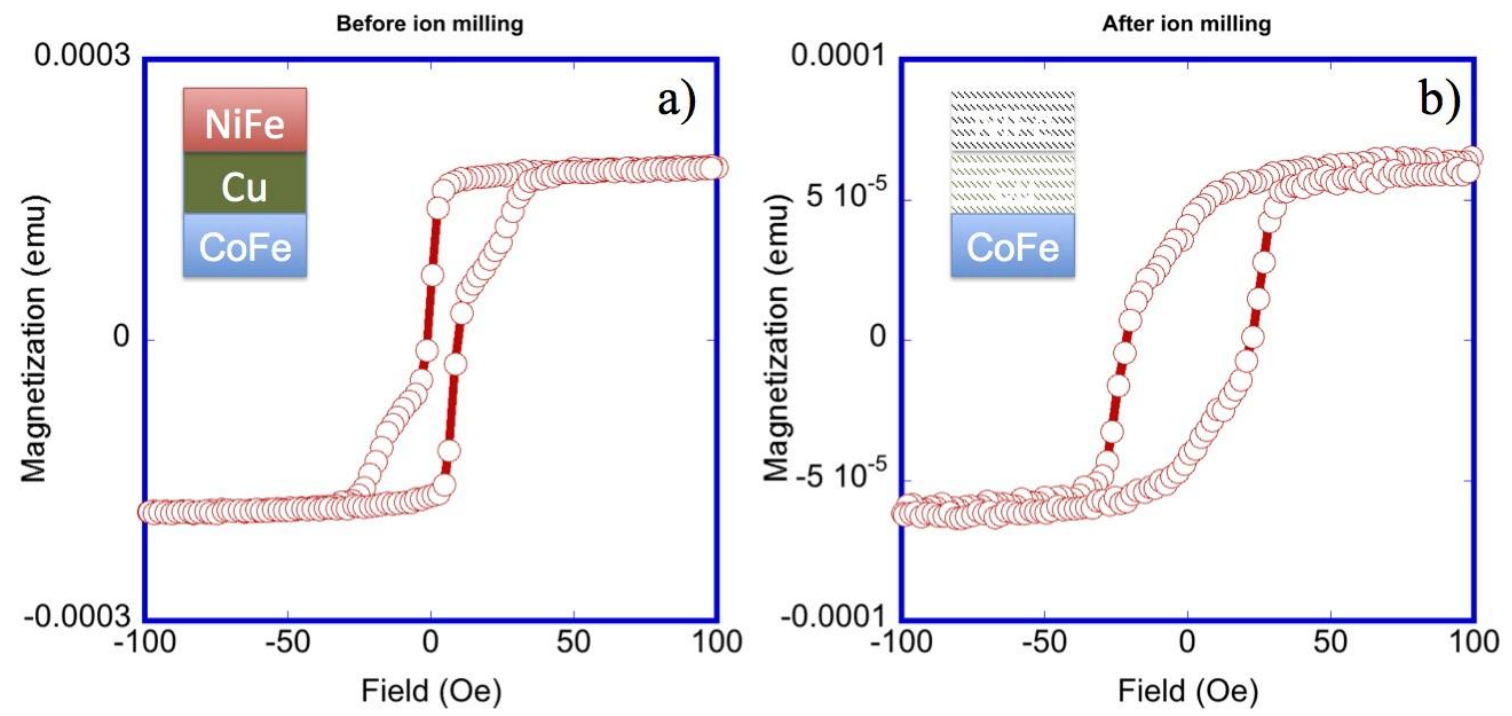

Figure 6.6 To precisely monitor the ion-milling, the hysteresis loop of the test sample has been measured before and after etching. Quantitative information such as coercive fields, magnetization $\left(M_{\mathrm{NiFe}}, M_{\mathrm{CoFe}}\right)$ can be extracted from the Hloop, which are used to determine the time for the etching endpoint. (a) The hysteresis loop measured before ion milling. (b) The hysteresis loop measured after the layers above CoFe have been removed. 
In this ion mill step, a common problem is the re-deposition of the etched materials. This means some of the milled material will deposit on the sidewalls of the pillar and create a short between the fixed layer and free layer. I explored three different exposure scenarios and developed an innovated way that could prevent the shunting on the sidewall. Figure 6.7 shows the SEM image of the photoresist after development for the three exposure scenarios. With an exposure time of $12 \mathrm{~s}$ at $7.0 \mathrm{~mW} / \mathrm{cm}^{2}$, a cylinder shape resist pillar is formed after development as shown in Figure 6.7(a). The diameter is slightly bigger than the pattern size on the mask $(500 \mathrm{~nm})$ due to the diffraction effect[165]. An example of re-deposition on the edge is shown in Figure 6.7 (a) inset. Comparatively, Figure 6.7 (b) shows the result when I over expose the photoresist. The photoresist is wider in diameter $(\sim 791.7 \mathrm{~nm})$ on the bottom than on the top. Since the ion mill at 0 angle, the widest diameter of the photoresist pillar will be directly transferred to the device pillar. As a result, the device pillar will have a much larger size than desired in this scenario. To address the re-deposition issue and to improve the device size, our innovated approach is to create an undercut on the photoresist by under-exposure. Figure 6.7 (c) demonstrates a V-neck shape photoresist pillar with an undercut on the bottom. The diameter on the bottom is $\sim 373.8$ $\mathrm{nm}$ and the largest diameter on the top gives a desired value $\sim 510.2 \mathrm{~nm}$. Most importantly, with this configuration, most of the re-deposited materials will be attached to the photoresist instead of falling to the sidewall of the pillar. This is demonstrated in Figure 6.7 (d), the ideal V-shape PR protects the bottom from the re-deposition by drawing the 
re-deposited materials on its surface leading to a slightly larger diameter $\sim 532.3 \mathrm{~nm}$ comparing with $510.2 \mathrm{~nm}$. The device pillar on the bottom is narrow and clean with a diameter $\sim 500 \mathrm{~nm}$. Those re-deposited materials on the PR are later on removed during liftoff.

The photoresist on the pillar is left without liftoff, so that during the subsequent passivation step the pillars will be protected from $\mathrm{SiO}_{2}$ deposition. The device diagram at this step is shown in Figure 6.8.

a)

\section{Exposure to UV : \\ $\mathrm{t}=12 \mathrm{~s} @$ $7.0 \mathrm{~mW} / \mathrm{cm}^{2}$}

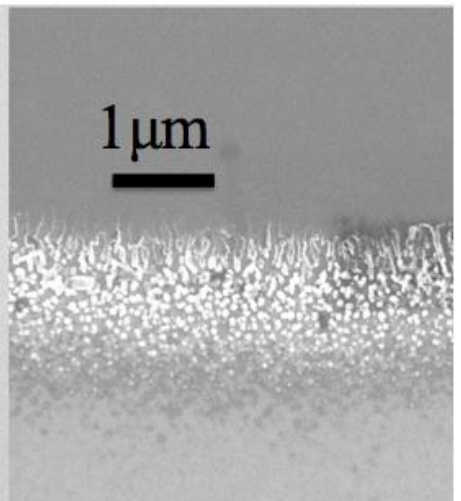

\section{7nm}

\begin{tabular}{|llll|}
$1 \mu \mathrm{m}$ & $\begin{array}{lll}\text { EHT }=1.50 \mathrm{kV} & \text { Signal } A=S E 2 & \text { Date }: 2 \text { Aug } 2015 \\
\text { WD }=6.6 \mathrm{~mm} & \text { Photo No. }=3354 & \text { Time }: 17: 26: 38\end{array}$ \\
\hline
\end{tabular}


b)

\section{Over \\ Exposure to \\ UV : \\ $\mathbf{t}>12 \mathrm{~s} @$ \\ $7.0 \mathrm{~mW} / \mathrm{cm}^{2}$}

\section{$791.7 \mathrm{~nm}$}

$\begin{array}{llll}1 \mu \mathrm{m} & \text { EHT }=2.50 \mathrm{kV} & \text { Signal } A=\text { SE2 } & \text { Date }: 27 \text { Sep } 2015 \\ \text { WD }=6.5 \mathrm{~mm} & \text { Photo No. }=3925 & \text { Time }: 16: 15: 28\end{array}$

c)

\section{Under \\ Exposure to \\ UV : \\ $\mathbf{t}<12 \mathrm{~s} @$ \\ $7.0 \mathrm{~mW} / \mathrm{cm}^{2}$}

$373.8 \mathrm{~nm}$ 
d)

\section{Before Etching After Etching}

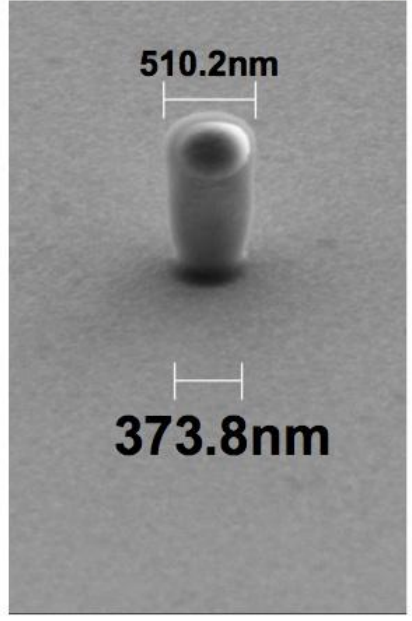

$532.3 \mathrm{~nm}$

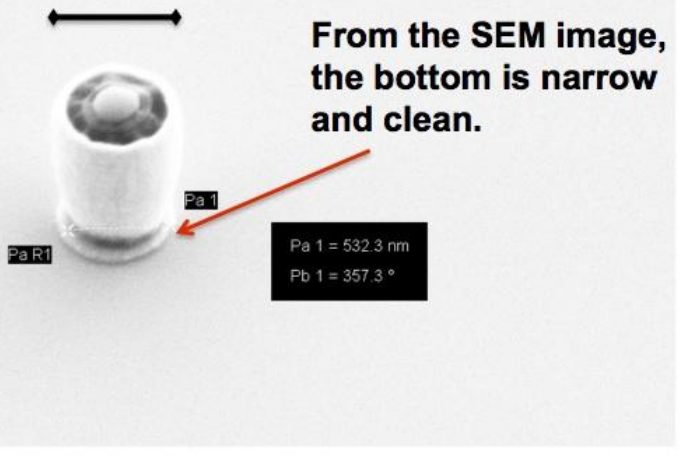

Figure 6.7 (a) A SEM image showing the photoresist pillar after development. The inset shows the sidewall re-deposition after ion milling. (b) A resist pillar with over-exposure. (c) A V-neck shape resist pillar with under-exposure time. (d) Comparison of the same resist pillar before and after etching.

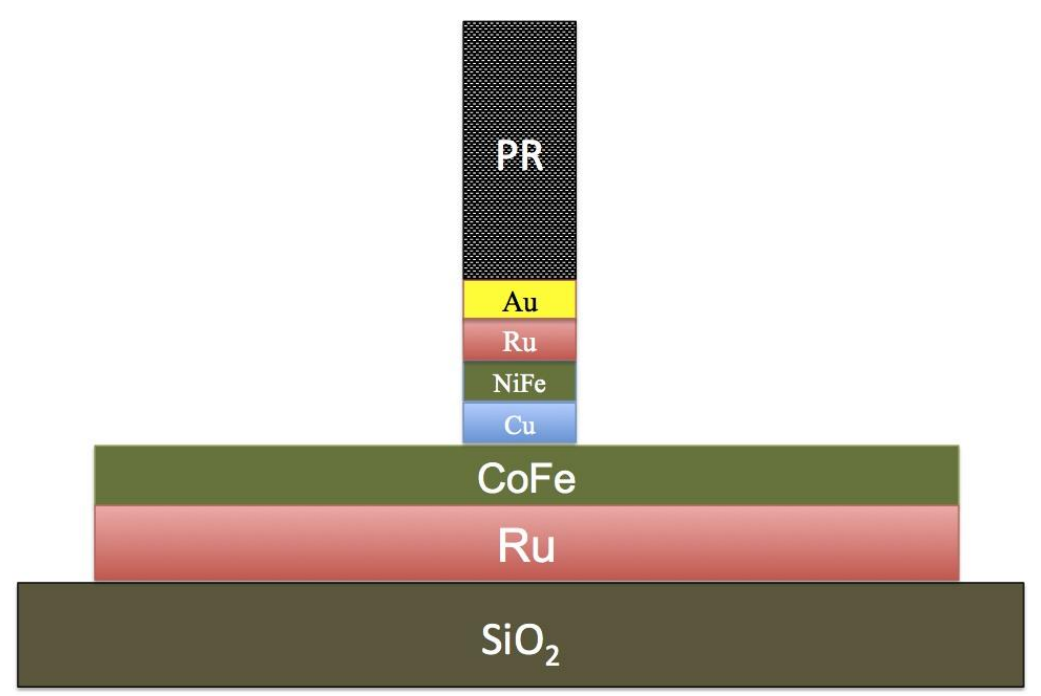

Figure 6.8 Schematic diagram showing the cross section of the device. The photoresist 
(PR) remains above the pillar after etching.

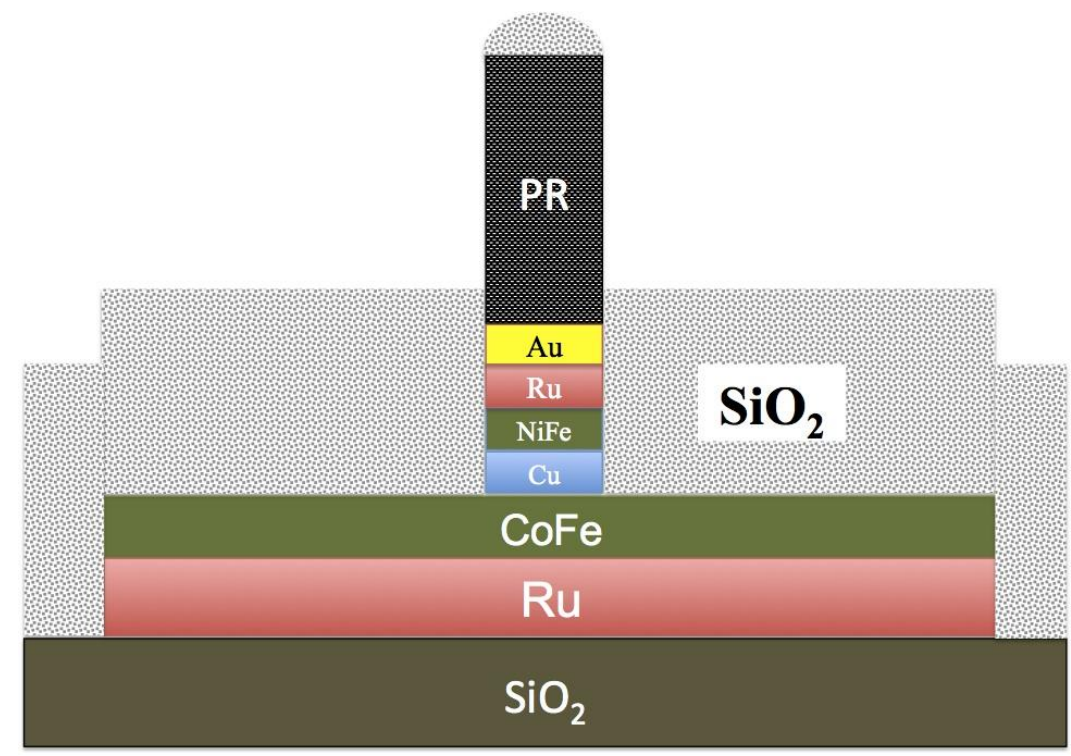

Figure 6.9 Deposition of $\mathrm{SiO}_{2}$ to cover the bottom electrodes and to insulate the sidewall of the device pillar.

\section{$\mathrm{SiO}_{2}$ passivation}

After the bottom contacts and the nano-pillar have been defined, the next step is to surround the pillar with an insulating layer. For the bulk of the insulation layer we deposit a $280 \mathrm{~nm} \mathrm{SiO} 2$ layer with the $\mathrm{AJA} \mathrm{SiO}_{2}$ sputtering system in $\mathrm{UVa}$ clean room. The deposition rate is controlled to be very slow $(1.2 \mathrm{~nm} / \mathrm{min})$ to enable a smooth $\mathrm{SiO}_{2}$ surface and most importantly to produce a high quality insulating layer without pinholes. Figure 6.9 shows a schematic cross-section of the device after passivation. 


\section{Liftoff}

Photoresist, after the previous two steps is especially hard to remove due to that first it has been heated during ion milling and $\mathrm{SiO}_{2}$, secondly, part of the resist on top of the nano-pillar is now surrounded by $\mathrm{SiO}_{2}$ (As shown in the schematic Figure 6.9). As a result a more sophisticated resist striping process has been developed. First the wafer is soaked for 60 minutes in N-Methylpy (NMP) solution that is put on a $120{ }^{\circ} \mathrm{C}$ hotplate with a stirring bar. The photoresist will gradually be dissolved in the NMP solution. After cleaning by DI water, this soaking process is repeated 3-4 times. The wafer is then transferred into a beaker filled with Ethylene glycol solution and a Q-tip is utilized to gently wipe the wafer surface within the beaker so that the photoresist residue can be physically removed. This process is repeated with new Q-tip as needed. After the Q-tip process, the wafer is soaked in NMP again on hot plate for another 60 minutes. Following this, the wafer is removed, thoroughly cleaned with DI water, and blown dry. Finally, the $\mathrm{O}_{2}$ plasma will be applied to further remove the photoresist residue. Figure 6.10 clearly shows the SEM side-view and top-view image of the device after the photoresist has been removed. 
Side View

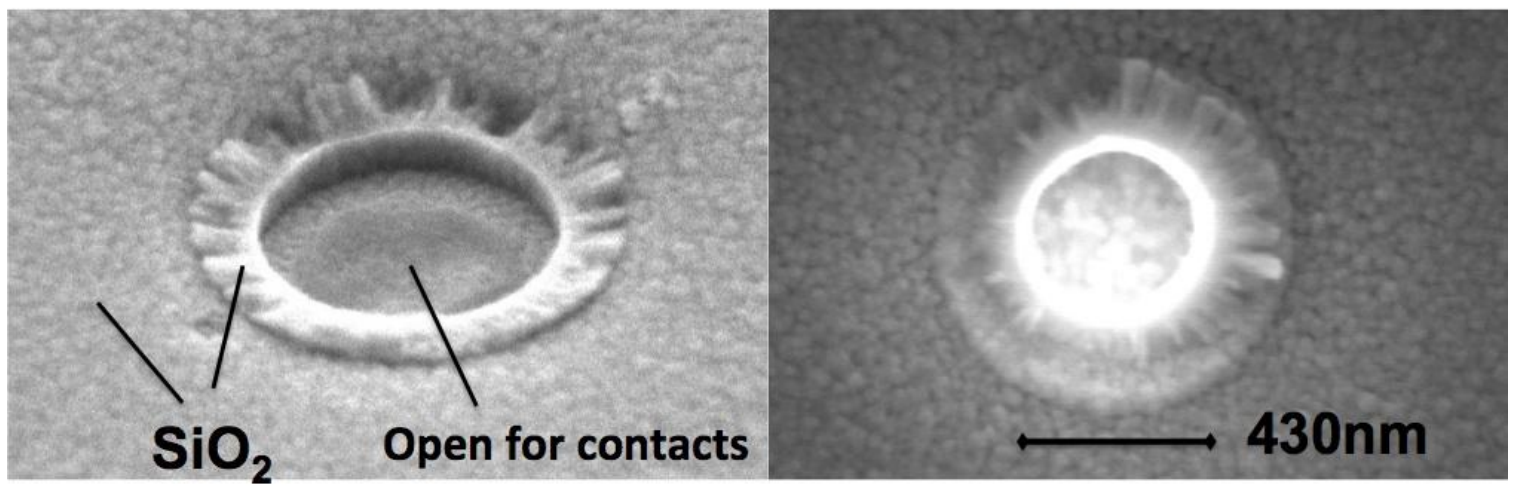

Figure 6.10 Side view and top view showing a crater-shape opening for the device pillar

The brighter circle seen in the top-view is due to the charge accumulation during the SEM scan.

\section{$\mathrm{SiO}_{2}$ via photolithography}

The $\mathrm{SiO}_{2}$ above the bonding pads must be removed to open a hole for the top contacts. A photolithography step is performed to leave unprotected the area above the bonding pads. The mask pattern used for the via is shown in Figure 6.3(c). After copying those patterns to the photoresist, the exposed $\mathrm{SiO}_{2}$ is then removed using buffered oxide etch (1:10) which is primarily 1 part hydrofluoric (HF) acid to 10 parts dilutant. The etch rate in this solution is $\sim 200 \mathrm{~nm} / \mathrm{min}$ so one and half-minute etch is usually sufficient. The photoresist is then removed by acetone and MARCH after wet etching. Figure 6.11 shows the schematic of the via as well as the device at this point in the fabrication process. 


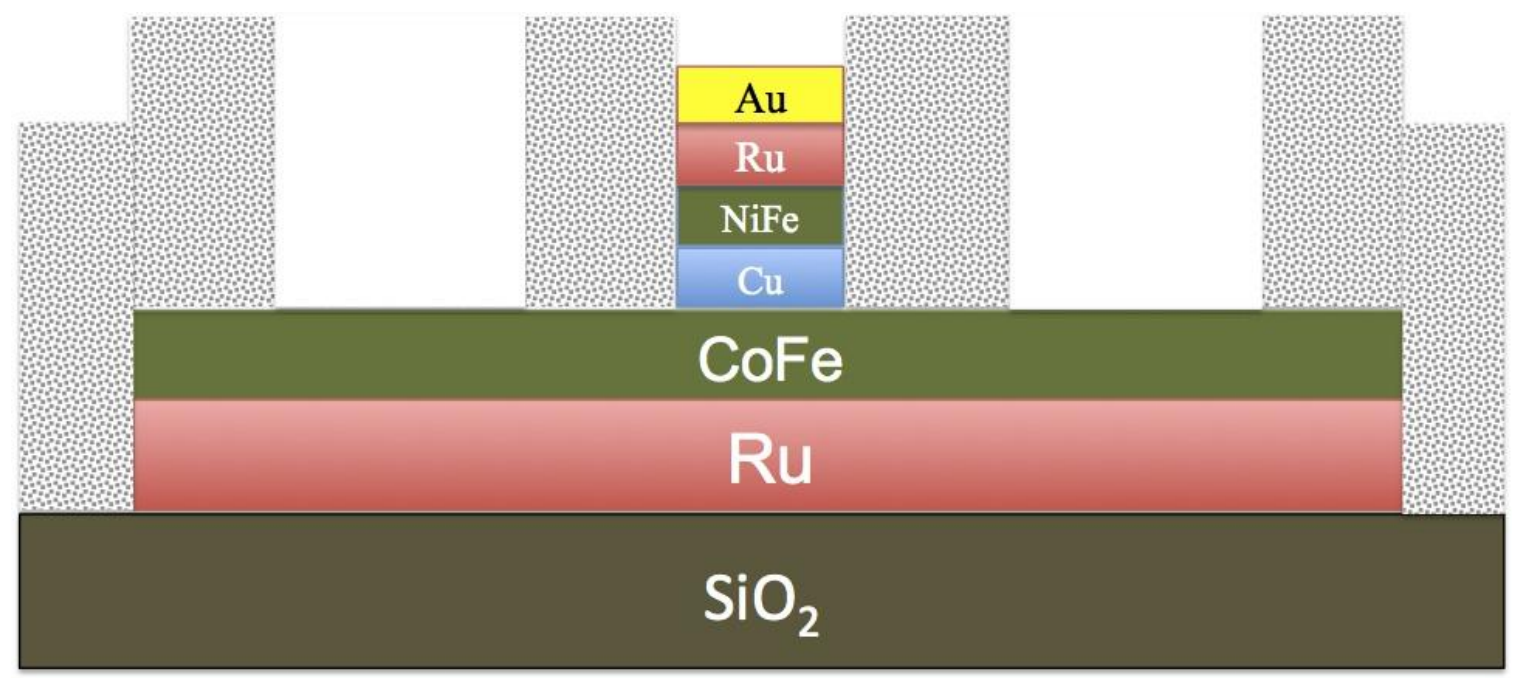

Figure 6.11 Schematic showing the device after the $\mathrm{SiO}_{2}$ over the bonding pads has been removed with a buffered oxide etch (BOE) etch.

\section{Au plating for top contacts}

For the purpose of RF measurement, the top contacts need to be thick (micro meter) to reduce the transmission line attenuation. So instead of using e-beam Au evaporation, I did Au electrochemical plating to deposit a $\sim 4 \mu \mathrm{m}$ Au layer which served as the top contacts. Before the Au plating, a 10nm Ti and $10 \mathrm{~nm} \mathrm{Au} \mathrm{seed} \mathrm{layer} \mathrm{had} \mathrm{been} \mathrm{deposited} \mathrm{across} \mathrm{the}$ whole wafer by sputtering. The seed layer was necessary here as a bottom electrode for the electrodepostion but would later be removed so that the pillar and the bonding pads won't be shorted. Following that, a photolithography was performed based on mask layer 4 (Figure 6.3(d)) to leave the top contacts area unprotected. The wafer was then transferred to a plate station and $4 \mu \mathrm{m}$ thick of Au will be electrodeposited on those unprotected 
contact areas. After Au plating, the resist on the wafer was removed by $\mathrm{O}_{2}$ plasma by leaving the wafer in MARCH overnight with a power of $200 \mathrm{~W}$.

The last step is to remove the seed layer so that the top contacts won't connect to the bottom bonding pads. This is done by selective wet etch for Au and Ti. Our HG-800 Au wet etch is composed of I $10 \mathrm{~g}, \mathrm{INH}_{4} 40 \mathrm{~g}$, DI water $900 \mathrm{ml}$, and ethanol $1100 \mathrm{ml}$. The etchant requires approximately 20 seconds to remove $10 \mathrm{~nm}$ of Au seed layer. Since the plated Au layer is sufficiently thick, so an over-etching will help to completely remove the Au seed layer without affecting the top/bottom bonding pads. The Ti seed layer is then removed by 30 s of BOE wet etch. Finally, we perform a conductivity check to ensure the seed layer has been removed completely. Figure 6.12 shows the completed devices and

Table 6-1 provides a summary listing the major processing steps that were outlined in this section.

\subsubsection{Device tracking and quality control}

Fabricating $500 \mathrm{~nm}$ nanostructures by photolithography is a long and difficult process.

The procedure just described contains 4 photolithography steps, 1 liftoff step, 2 evaporation steps, 2 sputtering steps, 1 ion milling steps and 3 wet etch steps. Clearly, such a long process has the potential to have extremely low yield, so to improve yield, extra precautions must be taken. To insure decent device yields every step of the fabrication 
process must be tested and tracked.

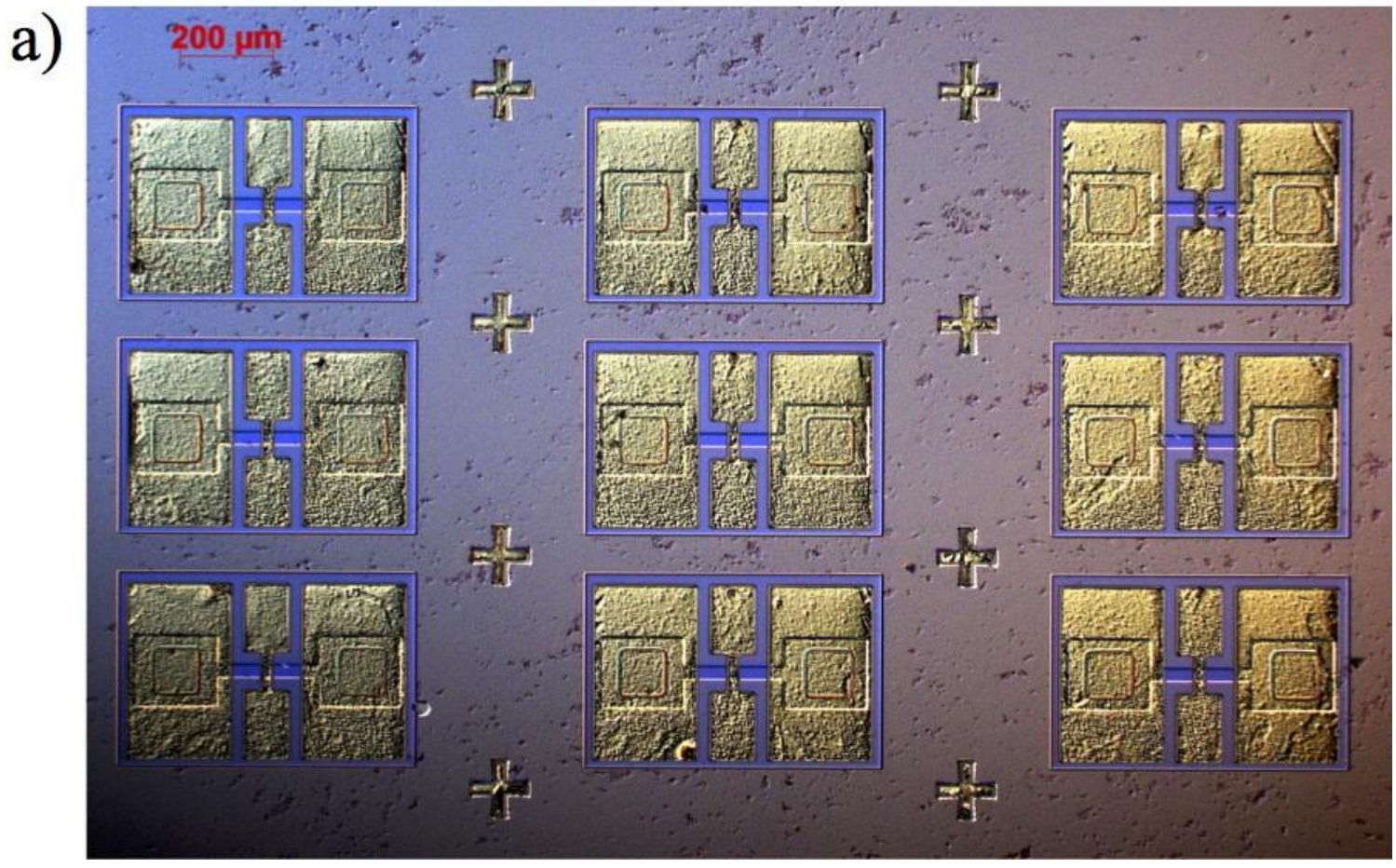

b)

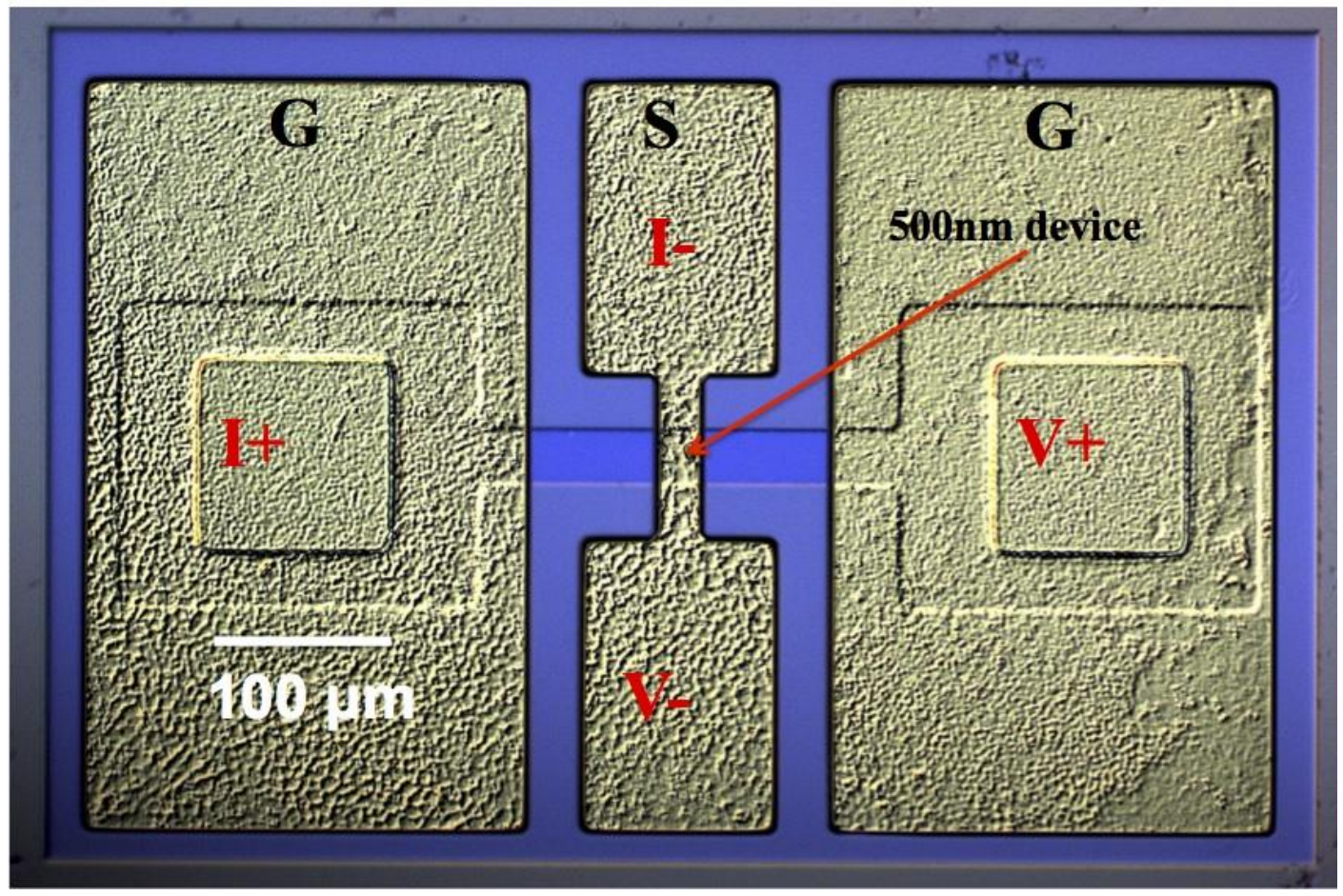

Figure 6.12 (a) A microscope image showing an array of devices after the seed layer have been removed. (b) The STNO device with special designed contact pads. 
Table 6-1 Outline of the steps used to make CPP nano-pillars at UVa

\begin{tabular}{|c|c|}
\hline Step & Process \\
\hline 1 & Sputtering of multilayer structures by BTIBD \\
\hline 2 & Evaporation of $\mathrm{Au} / \mathrm{Ti}$ \\
\hline 3 & Bottom electrode defining photolithography \\
\hline 4 & Ion-milling \\
\hline 5 & Nano-pillar defining photolithography \\
\hline 6 & Ion-milling \\
\hline 7 & $\mathrm{SiO}_{2}$ passivation \\
\hline 8 & Liftoff \\
\hline 9 & $\mathrm{SiO}_{2}$ via etch photolithography \\
\hline 10 & $\mathrm{SiO}_{2}$ via wet etching \\
\hline 11 & Sputtering of seed layer $\mathrm{Au} / \mathrm{Ti}$ \\
\hline 12 & Au plating photolithography \\
\hline 13 & Au plating of top electrode \\
\hline 14 & Liftoff \\
\hline 15 & Seed layer removing by wet etching \\
\hline
\end{tabular}


After patterns are exposed by photolithography, the patterns need to be inspected to ensure alignment and pattern quality. Secondly, etch depths and deposition thicknesses need to be carefully measured and tracked. Also, across-wafer uniformity needs to be measured and tracked.

It is noteworthy to point out that the photoresist residue could severely affect the device performance if it was not removed completely. For example, an extremely prolonged procedure was carried out during the liftoff step in order to eliminate the resist on top of the nano-pillar. However, if not removed completely, the resist will act as an insulating layer between the pillar and the top contacts leading to an open circuit.

The way we test the quality of the fabrication is to measure the electrical transport properties of the devices. First, the full wafer of devices is separated into smaller chips by a dicing saw (7100 HM, Kulicke \& Soffa industries inc.). The devices are labeled based on the columns, rows and chips' number. A preliminary resistivity measurement is carried out by probe station, which gives information including short circuit, open circuit or a resistivity value that is close to the multilayer's material properties $\left(\sim 1.25 \times 10^{-7} \Omega \cdot \mathrm{m}\right)$.

GMR measurement is the most effective way to check the quality of fabrication. In principle, the GMR ratio with CPP patterning should be higher than the GMR measured with CIP geometry. However, a few steps in the processing could lead to a smaller GMR ratio. Firstly, the re-deposition on the sidewall of the nano-pillar during the etching step causes a shunt. As a result, not all the current is channeled through the magnetic 
multi-layers, resulting in low GMR. Secondly, the etching might cause damage to the magnetic materials leading to a low spin-polarization, which lowers the GMR ratio[166]. Finally, the photoresist residue on top of the pillar will increase the resistance of the device and decrease the GMR ratio. For instance, we measured the resistance versus temperature from $300 \mathrm{~K}$ to $50 \mathrm{~K}$ as shown in Figure 6.13. For the devices we have, a linear metallic $\mathrm{R}$ versus T curve shown in Figure 6.13(a) is expected. However, for some of the devices, we observed a significant increase of $\mathrm{R}$ as the temperature decrease, for example the device shown in Figure 6.13(b), which is more of a semiconductor or insulator behavior. A very thin layer of photoresist residue is believed to remain above the pillar resulting in the increase of resistance at lower temperature.
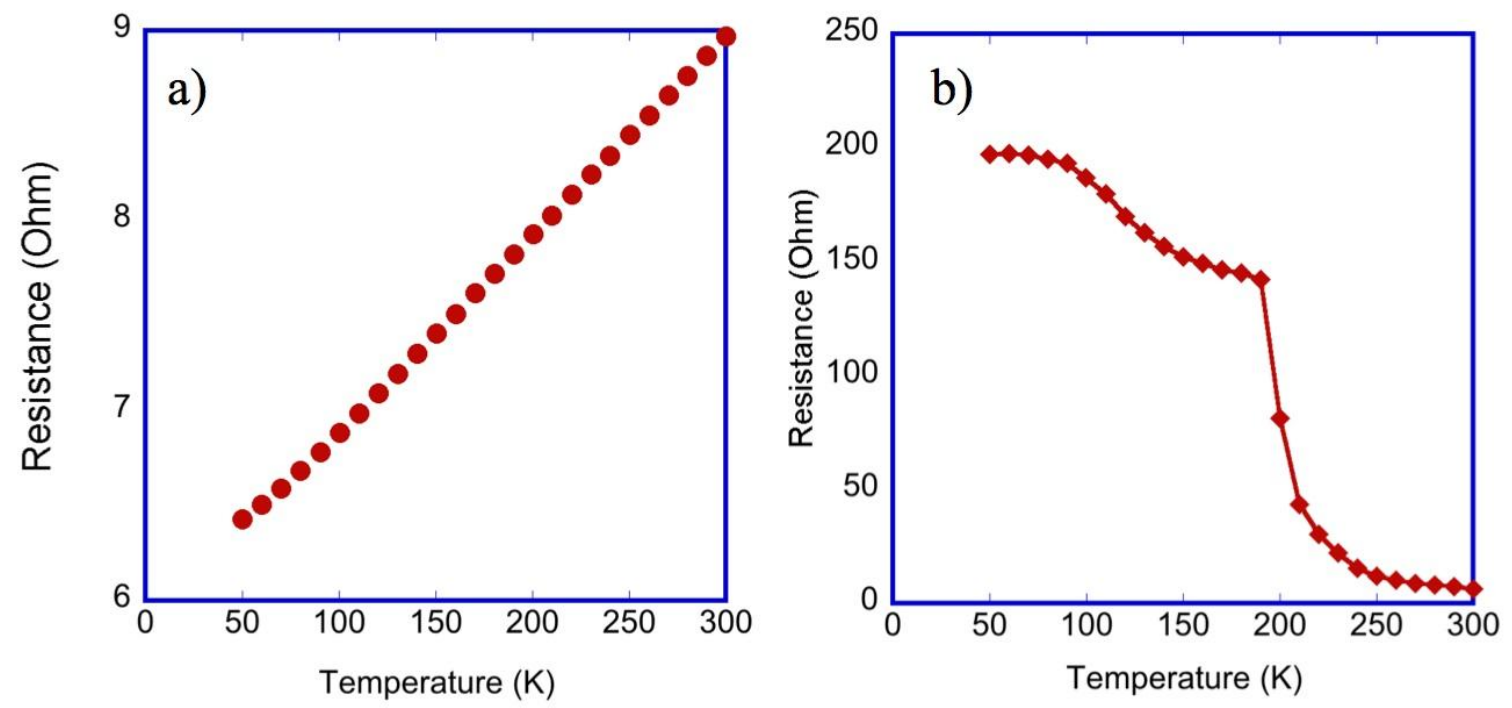

Figure 6.13 Resistance versus temperature curve is used to evaluate the fabrication quality. (a) A linear decreasing curve indicates a metallic structure. (b) The abnormal R-T 
curve could indicate the remaining of PR above the pillar. The sharp jump on $\mathrm{R}$ is coming from the loose contact of wire bonding.

\subsubsection{Another example: CPP patterning of magnetic tunnel junctions}

As mentioned before, the CIP geometry has the limitation that it cannot be applied to measure the TMR. Therefore, a CPP micro-fabrication processing for magnetic tunnel junctions (MTJs) has been developed to examine the TMR through transport measurements. The patterning process was carried out by UV photolithography techniques using a chromium mask as shown in Figure 6.14. The MTJ pillars are defined with various aspect ratios.

Figure 6.15 shows the flow chart for the critical steps in the processes. The bottom contact is defined by a positive photoresist. Then the $\mathrm{Ar}^{+}$ion milling step was carried out to etch the film down to the substrate. The junction was defined, and then a $280 \mathrm{~nm} \mathrm{SiO}_{2}$ insulating layer was deposited by sputtering. This via was defined by Figure 6.14(c) and etched by BOE. Finally, Au contacts were defined using lift-off technique. Figure 6.16 shows the patterned MTJ pillars observed by optical microscopy.

To test the quality of the MTJ fabrication, similar methods to those mentioned in Section 6.2.3 have been carried out. However, we need to take extra precaution to prevent the static charges during the measurement. A pulse shock from a static charge can easily 
break down the MTJ barrier and severely reduce the yield of the fabrication. A switch box for probe-station has been designed to ground the probes before any measurement.

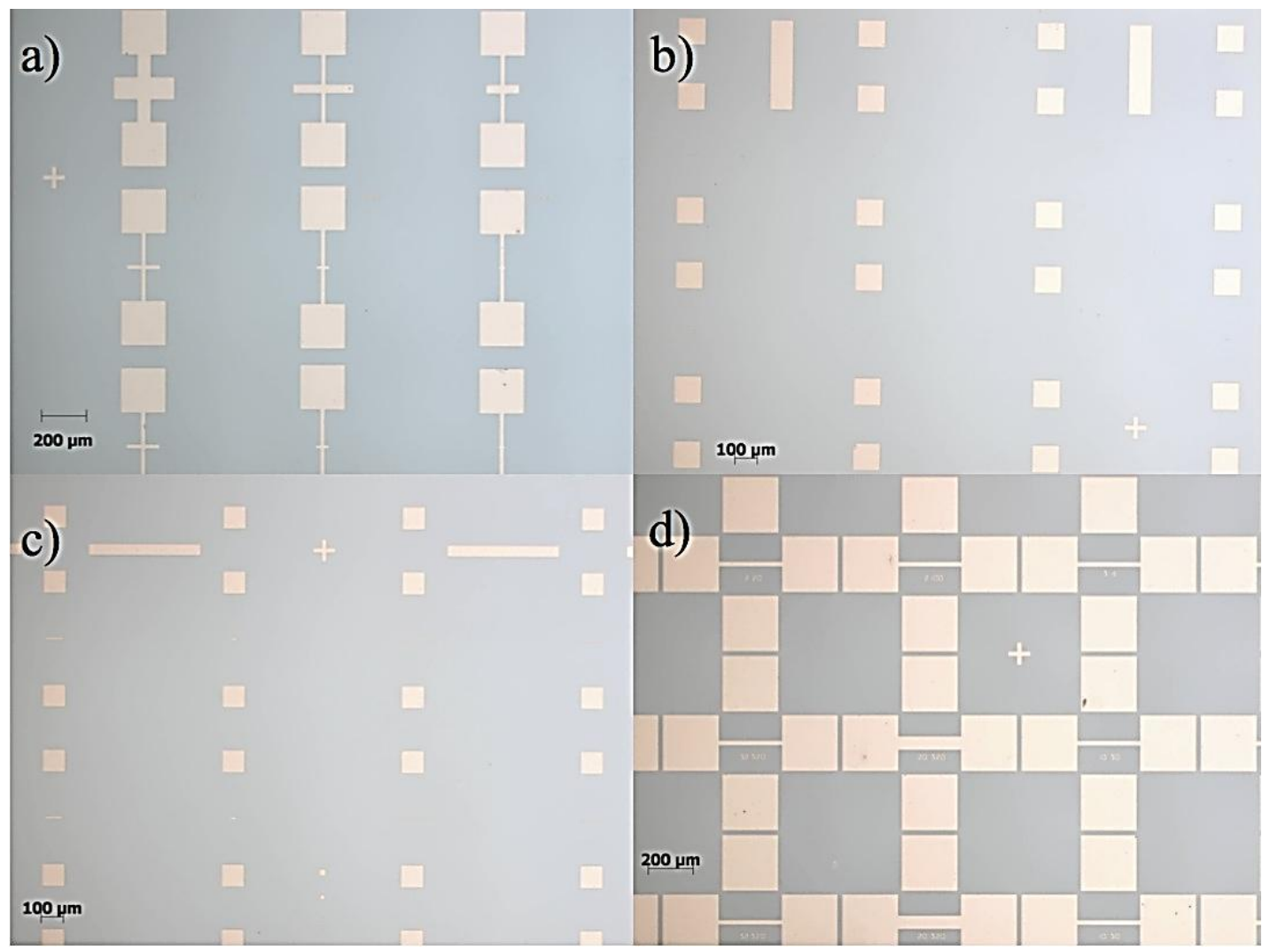

Figure 6.14 MTJ photolithography mask. (a) Layer 1 is to define the bottom contacts. (b) MTJ pillars with various aspect ratios are defined by layer 2. (c) Layer 3 is to open the via area in the passivation layer. (d) Layer 4, to define the top contact. 


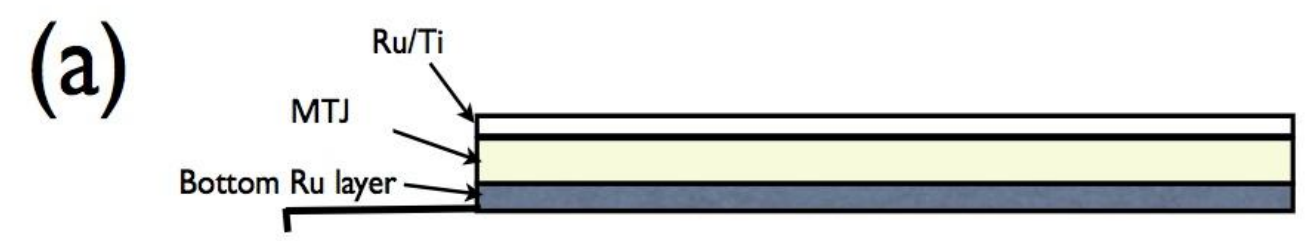

(b)

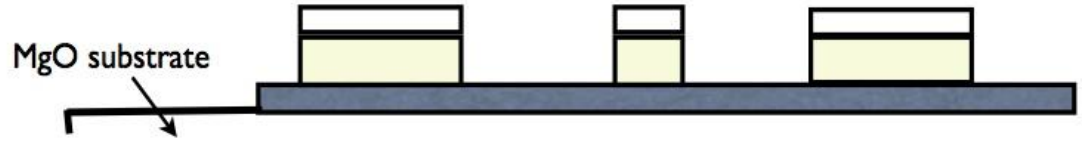

(c)

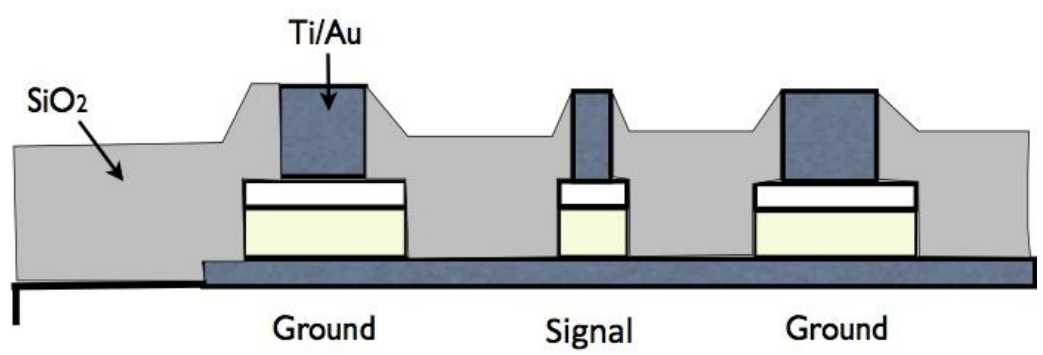

Figure 6.15 Flow chart of MTJ fabrication process: (a) Definition of the bottom contact.

(b) Definition of the junction through ion milling down to the bottom Ru contact layer. (c)

Definition of the via and top contact pads by $\mathrm{SiO}_{2}$ passivation and $\mathrm{Au}$ evaporation. 


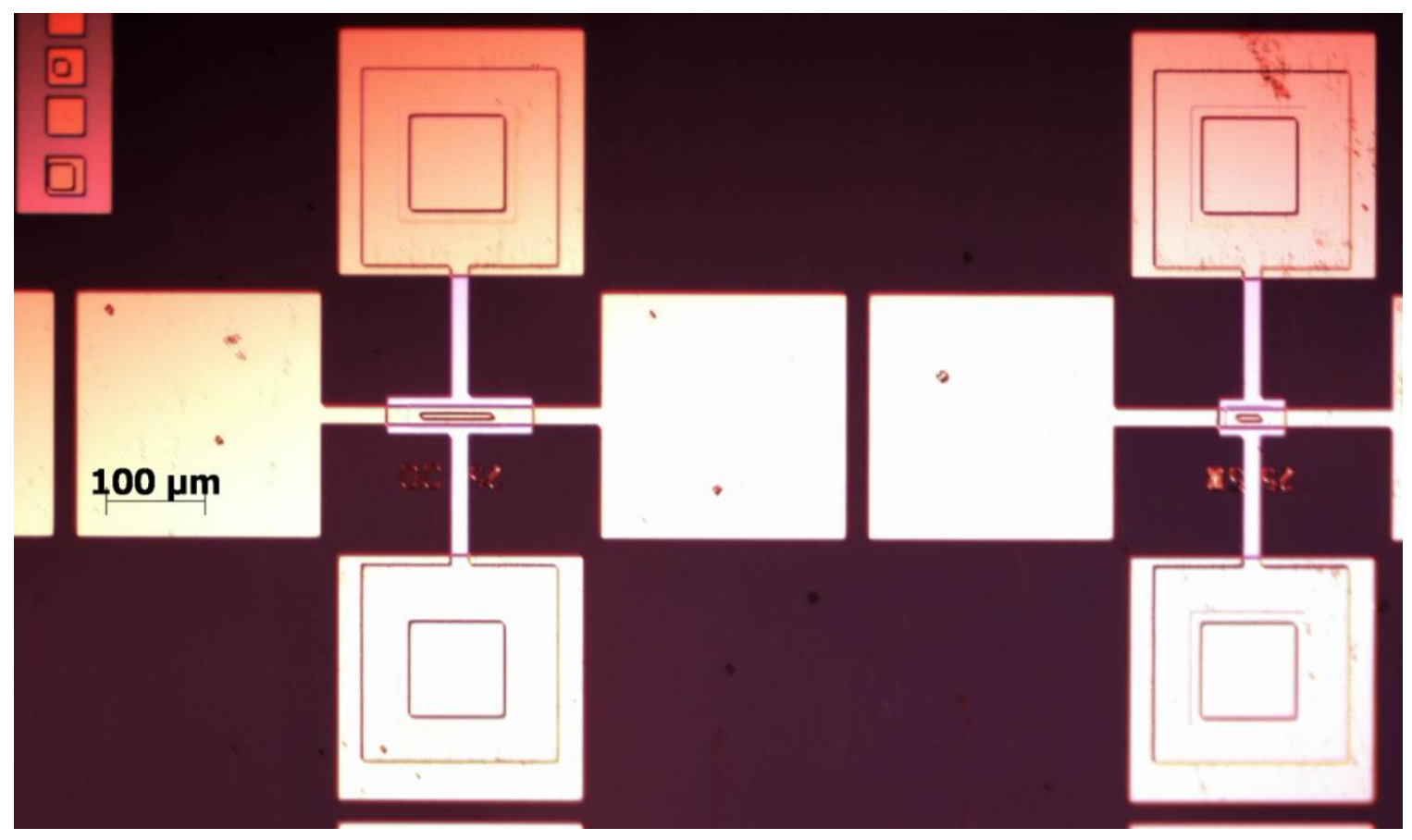

Figure 6.16 Optical image showing elongated MTJ pillars with different aspect ratio of $10 \times 50 \mu \mathrm{m}^{2}($ Left $)$ and $10 \times 20 \mu \mathrm{m}^{2}($ Right $)$.

\subsection{Limitations of the CPP fabrication by photolithography}

This CPP fabrication process contains certain limitations. Firstly, as the size on the mask shrinks below $500 \mathrm{~nm}$, interference presents a challenge to the process. As the mask serves as a diffraction grating, the light that passes through the mask gets split into many separate light beams which start interfering with each other, creating an optical pattern on the wafer surface that differs from the pattern on the mask. Secondly, the fabrication includes different tools and certain complicated steps, which increase the probability for 
non-uniformity.

\subsection{CPP fabrication summary}

In this Chapter, I discussed the fabrication techniques that I used to fabricate $500 \mathrm{~nm}$ diameters CPP magnetic nanostructures in a photolithographic process. Comparing to the conventional nanostructure patterning using electron beam lithography (EBL), our fabrication process is more cost effective and time-efficient. Besides, this fabrication process can be utilized to fabricate nanostructures in a much larger scale ( 2 inch wafer in our case), which is beyond the capability of EBL. The innovated method to make an undercut on the photoresist pillar can be used to effectively prevent the sidewall re-deposition during the ion-milling process.

With these fabrication techniques we can now study CPP transport for magnetic nanostructures. This opens up a whole range of possibilities for the study of spin-transfer torque switching and oscillation. Since the critical current density for the spin transfer

torque effect is on the order of $10^{7} \mathrm{~A} / \mathrm{cm}^{2}$ [4], a small size device could tremendously bring down the magnitude of the critical current. 


\section{Chapter 7 Spin transfer in $\mathrm{Ni}_{80} \mathrm{Fe}_{20}$ based magnetic multilayers}

\subsection{Introduction}

It has been suggested that two nano-contact STNOs in close proximity could mutually phase-lock and increase the output power; however phase-locking of more than two STNOs remains technologically challenging [57, 58, 167-169]. Instead of putting an array of STNOs nano-magnets together, we propose to make use of larger sized magnets in the hope that synchronization of multiple domains could lead to higher output power, and firstly we demonstrated that spin-transfer torque could be used to efficiently induce magnetization switching and oscillation in $500 \mathrm{~nm}$ large size devices. For large size device, our simulation results have shown that the non-uniform oscillations tend to synchronize with each other and generate coherent oscillation. In addition, large sized nano-magnets can be fabricated more cost-effectively through photolithography rather than using electron beam lithography. In this chapter I first discuss the magnetic static properties of the multilayer continuous films and the patterned device. Then I discuss the dynamic properties and demonstrated pure DC current induced magnetic reversal and oscillation on $500 \mathrm{~nm}$ size devices made from those films. Micromagnetic simulations are discussed at the end. 


\section{2 $\mathrm{Ni}_{80} \mathrm{Fe}_{20} / \mathrm{Cu} / \mathrm{Co}_{90} \mathrm{Fe}_{10}$ Multilayer}

A $\mathrm{SiO}_{2}$ (substrate)/5nm $\mathrm{Ru} / 6.5 \mathrm{~nm} \mathrm{Ni}_{80} \mathrm{Fe}_{20}$ (or $2 \mathrm{~nm} \mathrm{Co}_{90} \mathrm{Fe}_{10}$ )/5nm $\mathrm{Ru}$ film was fabricated to characterize the coercive field of $\mathrm{Ni}_{80} \mathrm{Fe}_{20}$ (or $\mathrm{Co}_{90} \mathrm{Fe}_{10}$ ). A pseudo spin valve (PSV) multilayer structure $\mathrm{SiO}_{2}$ (substrate) $/ \mathrm{Ru} / \mathrm{Co}{ }_{90} \mathrm{Fe}_{10} / \mathrm{Cu} / \mathrm{Ni}_{80} \mathrm{Fe}_{20} / \mathrm{Ru}$ was deposited to study the interlayer coupling. The $\mathrm{Cu}$ spacer thicknesses were varied from $1.2 \mathrm{~nm}$ to $10 \mathrm{~nm}$.

\subsubsection{Coercivity tuning for $\mathrm{Cog}_{90} \mathrm{Fe}_{10}$ reference layer}

In this system, we studied how the different thicknesses affect the coercive field $H_{c}$ for $\mathrm{Co}_{90} \mathrm{Fe}_{10}$ so that to optimize the thickness of the PSV reference layer. Figure 7.1(a) shows the hysteresis loops for $\mathrm{Co}_{90} \mathrm{Fe}_{10}$ single layer with thickness varying from $1.2 \mathrm{~nm}$ to $3.1 \mathrm{~nm}$. At thickness $d=1.2 \mathrm{~nm}$, there is no magnetization indicating a magnetic deadlayer due to the interlayer mixing between the ferromagnetic layer and the non-magnetic buffer layer/substrate. At $d=2.2 \mathrm{~nm}$, the hysteresis loop shows the maximum $H_{c} \sim 26$ Oe. With increased of the thickness, the $H_{c}$ decreased and $H_{c}$ eventually stabilized to $\sim 18$ Oe when the thickness was above $3.1 \mathrm{~nm}$ (shown on Figure 7.1(b)). When the film was ultra thin $(d=$ $2.2 \mathrm{~nm}$ ), the interface effect was dominant over the bulk effect. The pinning of domain walls caused by the interface roughness enhanced the coercivity [170]. To distinguish the coercivity of the CoFe layer from the coercivity of the NiFe layer $(H c \sim 5 \mathrm{Oe}), d=2.2 \mathrm{~nm}$ is used for the PSV structure. 

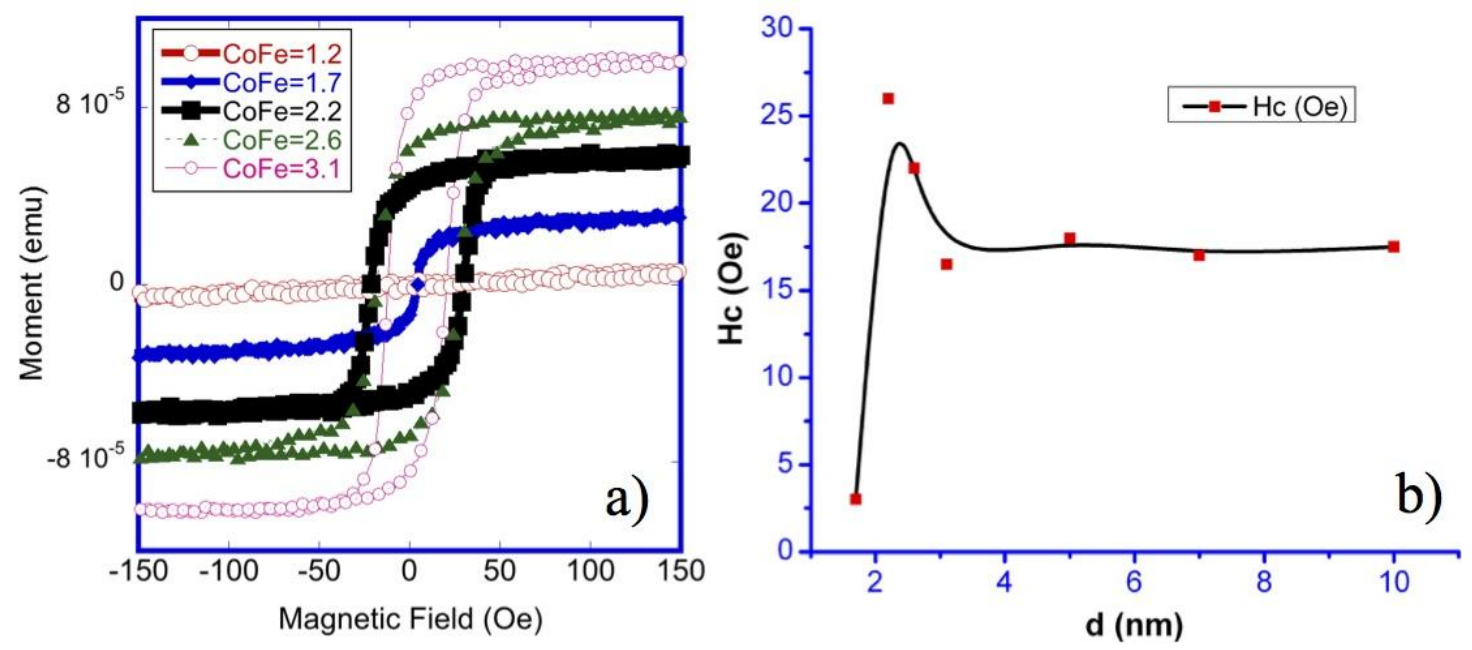

Figure 7.1 (a) The hysteresis loops showing thickness dependent $H_{c}$ for $\mathrm{Co}_{90} \mathrm{Fe}_{10}$ single layers. (b) The plot of coercivie fields for $\mathrm{Co}_{90} \mathrm{Fe}_{10}$ versus the thicknesses.

\subsubsection{Effect of the Cu spacer thickness}

Figure 7.2 shows the experimental magnetic hysteresis loops of the pseudo spin valve samples with different $\mathrm{Cu}$ spacer thicknesses. No clear separation on the $H_{c}$ is observed on samples (a) and (b), indicating the existence of a ferromagnetic coupling below the thickness $t=4 \mathrm{~nm}$. For sample (c) and (d), the larger $H_{c}=25 \mathrm{Oe}$, corresponding to $\mathrm{Co}_{90} \mathrm{Fe}_{10}$, while the smaller one $H_{c}=5$ Oe corresponds to $\mathrm{Ni}_{80} \mathrm{Fe}_{20}$ layer.

The MR ratios of the magnetic multilayers with different $\mathrm{Cu}$ spacer thicknesses are compared in Figure 7.3. An example of the measured $\mathrm{MR}$ with $\mathrm{Cu}=8 \mathrm{~nm}$ is shown in Fig. 7.4(a). The plot of MR ratio vs. Cu thickness is shown in Figure 7.3(b). For films with $\mathrm{Cu}=$ 
$3 \mathrm{~nm}$ and $4 \mathrm{~nm}$, they show much smaller MR ratio $(\sim 0.5 \%)$ due to the coupling between the reference layer and free layer. Note the non-square hysteresis loop indicated a small 90 degree coupling that led to the non-zero MR. The decrease of the MR for $t_{C u} \geq 5 \mathrm{~nm}$ can be understood as follows: the increase of spacer thickness increase the surface roughness, which weakens the spin dependent resistivity leading to a lower MR ratio [100]. Among the samples tested, $\mathrm{Cu}=5 \mathrm{~nm}$ not only shows a good separation on the hysteresis loop but also demonstrated the highest $M R$ ratio. As a result, multilayer $20 \mathrm{~nm} R u / 2.2 \mathrm{~nm}$ $\mathrm{Co}_{90} \mathrm{Fe}_{10} / 5 \mathrm{~nm} \boldsymbol{C u} / 6.5 \mathrm{~nm} \mathrm{Ni}{ }_{80} \mathrm{Fe}_{20} / 5 \mathrm{~nm} \mathrm{Ru}$ is used for the subsequence study. 

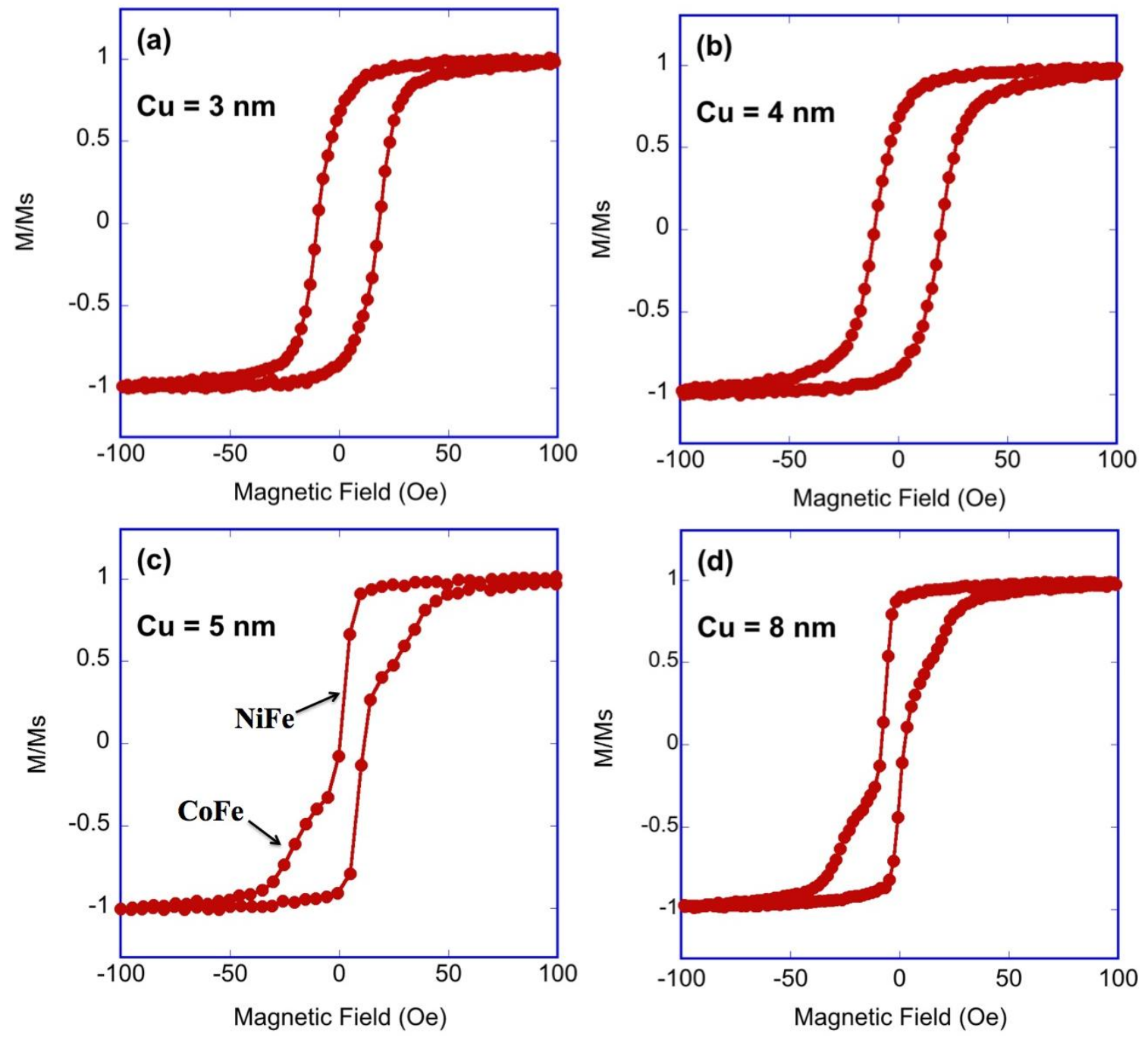

Figure 7.2 Experimental hysteresis loops of samples (a-d) with different $\mathrm{Cu}$ thickness varied from $3 \mathrm{~nm}$ to $8 \mathrm{~nm}$. The samples were measured at room temperature with the magnetic field applied in plane. The arrow marks the transition for $\mathrm{Ni}_{80} \mathrm{Fe}_{20}$ and $\mathrm{Co}_{90} \mathrm{Fe}_{10}$. 

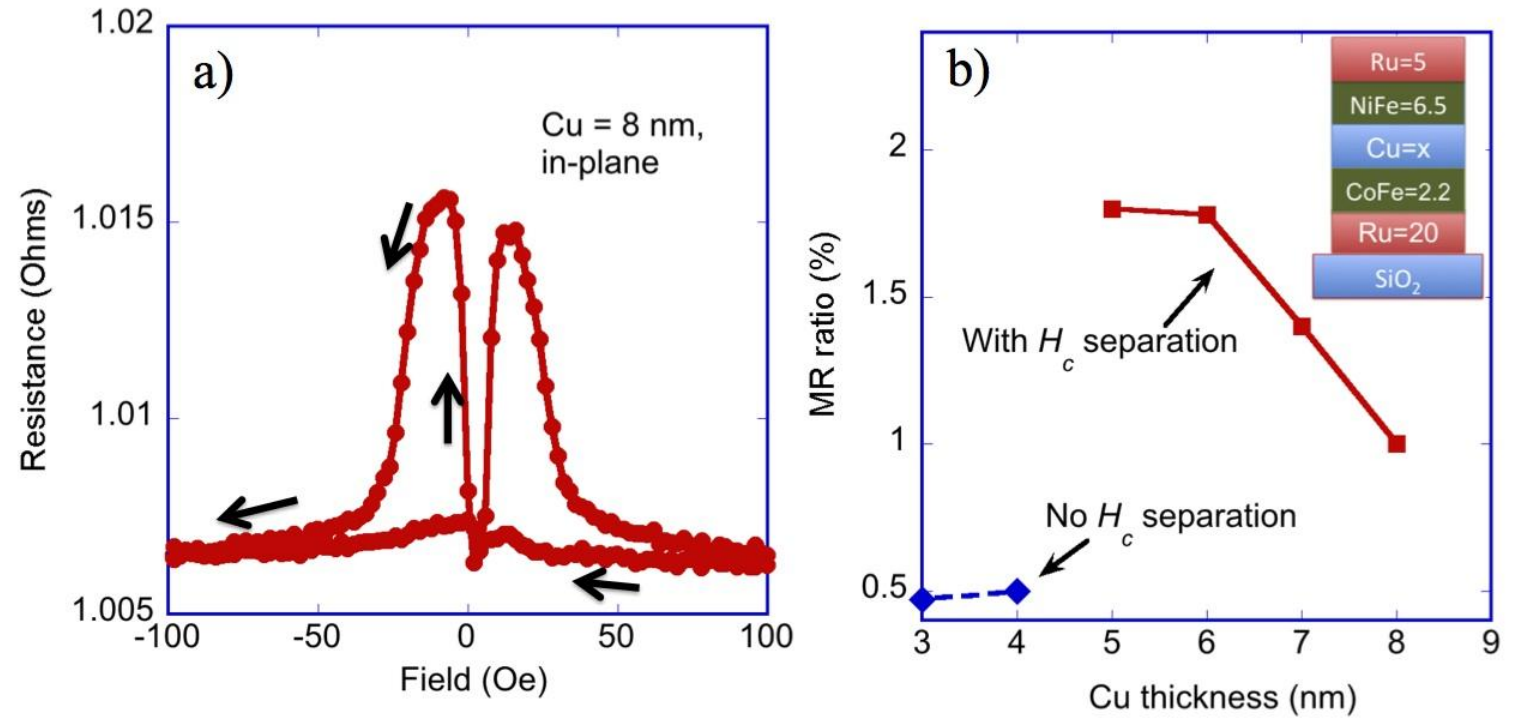

Figure 7.3 (a) Magnetoresistance measured by Van der Pauw configuration on the full film with $\mathrm{Cu}=8 \mathrm{~nm}$. (b) The MR ratios for the PSV (as shown inset) with different $\mathrm{Cu}$ thickness are plotted together for comparison.

\subsubsection{Magneto-transport behaviors of the PSV with out-of-plane}

\section{magnetic fields}

With the in-plane field, the $H_{c}$ separation is too small $(\sim 20 \mathrm{Oe})$ to be used in any practical applications. Besides, an all-in-plane structure gives small-angle oscillation and will limit the output power of the STNOs. To address these issues, we explored the magneto-transport behavior of the PSV with a perpendicular field.

The red curve in Figure 7.4 shows how the MR changes with an out-of-plane magnetic

field at room temperature. There are mainly two pairs of peaks on the curve, the low-field 
peaks and high-field peaks close to the kink point on the out-of-plane hysteresis loop [Figure 7.4 blue]. As for the low-field peaks, we zoom in it [Figure 7.5(a)] and compare it with the MR peaks measured under an in-plane field [Figure 7.5(b)]. By applying the out-of-plane field, a much lager coercive field separation between the $\mathrm{Co}_{90} \mathrm{Fe}_{10}(\sim 800 \mathrm{Oe})$ and $\mathrm{Ni}_{80} \mathrm{Fe}_{20}(\sim 100$ Oe) was achieved. The increase of the coercive fields can be understood in terms of the Stoner-Wohlfarth model [154].

To understand the magnetic configuration of the high-field peaks, we measured the rotation angle curves for both single layer $\mathrm{Ni}_{80} \mathrm{Fe}_{20}$ [Figure 7.5(c) blue] and single layer $\mathrm{Co}_{90} \mathrm{Fe}_{10}$ [Figure 7.5(c) red]. The rotation angle curve basically tells the information how big the angle of the magnetization rotates towards the out-of-plane direction. Both the $\mathrm{Ni}_{80} \mathrm{Fe}_{20}$ and $\mathrm{Co}_{90} \mathrm{Fe}_{10}$ layer' magnetization has been pulled out-of-plane at $30 \mathrm{kOe}$ field. As the field decreases, the $\mathrm{Co}_{90} \mathrm{Fe}_{10}$ layer's magnetization first starts to relax toward in-plane direction due to its large demagnetizing field. At $\sim 9 \mathrm{kOe}$, there is a maximum angle difference $\sim 50^{\circ}$ between the two layers' magnetization. The relative angle of the two layer's magnetization will then decrease with the further decreasing of the field and eventually reach 0 or $180^{\circ}$ as both layers relax back in-plane. We concluded that the high-field peaks were attributed to the maximum misalignment of the two layers' magnetization to the out-of-plane direction.

While both layers' magnetization tends to rotate toward the out-of-plane direction under a perpendicular field, the $\mathrm{Ni}_{80} \mathrm{Fe}_{20}$ layer's magnetization can be pulled more easily 
out of plane due to its smaller demagnetizing field $\left(4 \pi M_{S}\right)$. In comparison, a much larger external field was required to rotate the $\mathrm{Co}_{90} \mathrm{Fe}_{10}$ layer's magnetization. As a result, a pseudo hybrid configuration with an out-of-plane free layer and a near in-plane reference layer could be achieved at high perpendicular fields from $\sim 3 \mathrm{kOe}$ to $\sim 9 \mathrm{kOe}$. The magnetic hybrid configuration has been predicted to be more efficient at generating large amplitude precession and therefore increase the output power in STNOs [17, 171, 172].

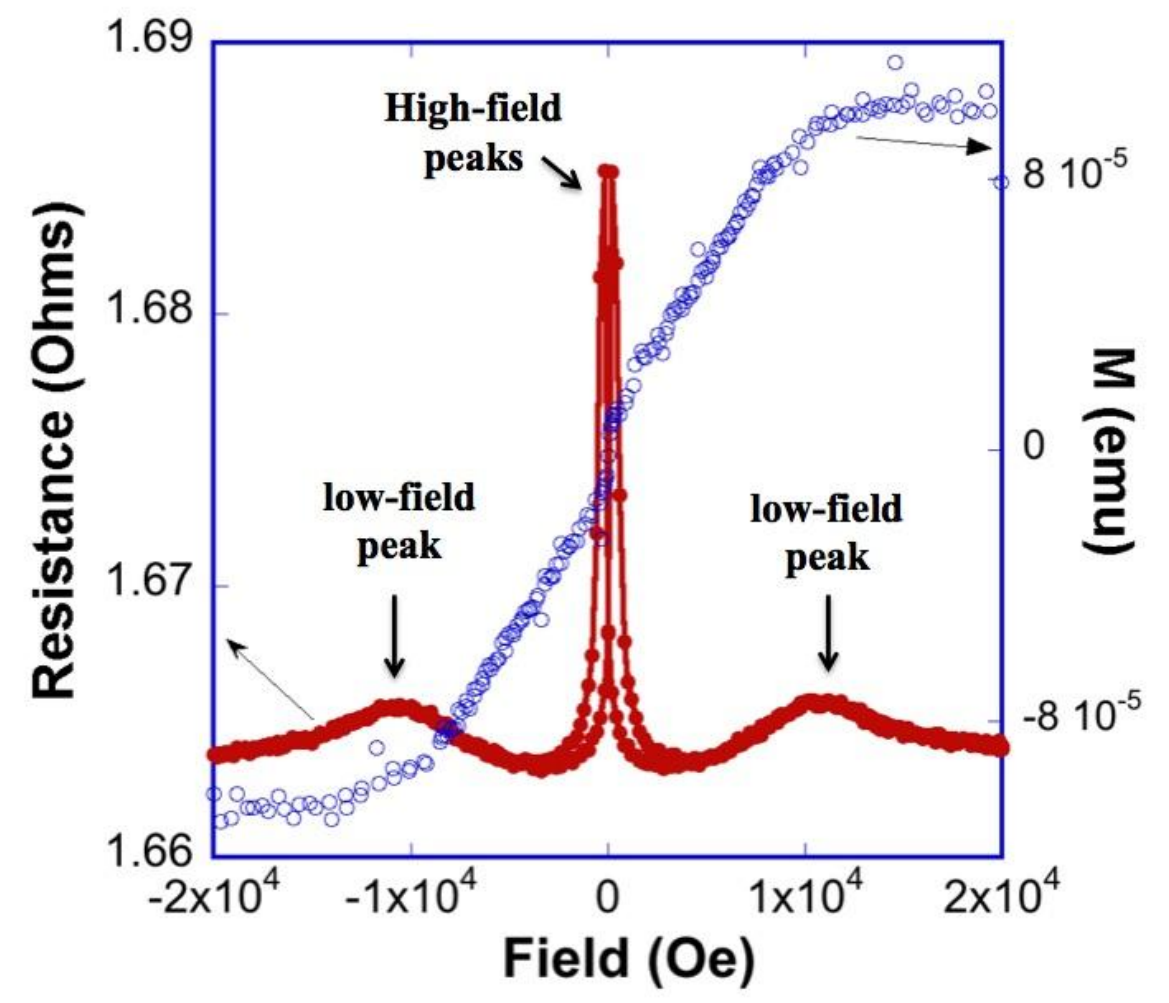

Figure 7.4 RT magnetoresistance curve (solid red) measured on the $20 \mathrm{~nm} \mathrm{Ru} / 2.2 \mathrm{~nm}$ $\mathrm{Co}_{90} \mathrm{Fe}_{10} / 5 \mathbf{n m} \boldsymbol{C u} / 6.5 \mathrm{~nm} \mathrm{Ni}_{80} \mathrm{Fe}_{20} / 5 \mathrm{~nm}$ Ru PSV structure with an out-of-plane magnetic field. The blue curve shows the corresponding hysteresis loop on the out-of-plane direction. 

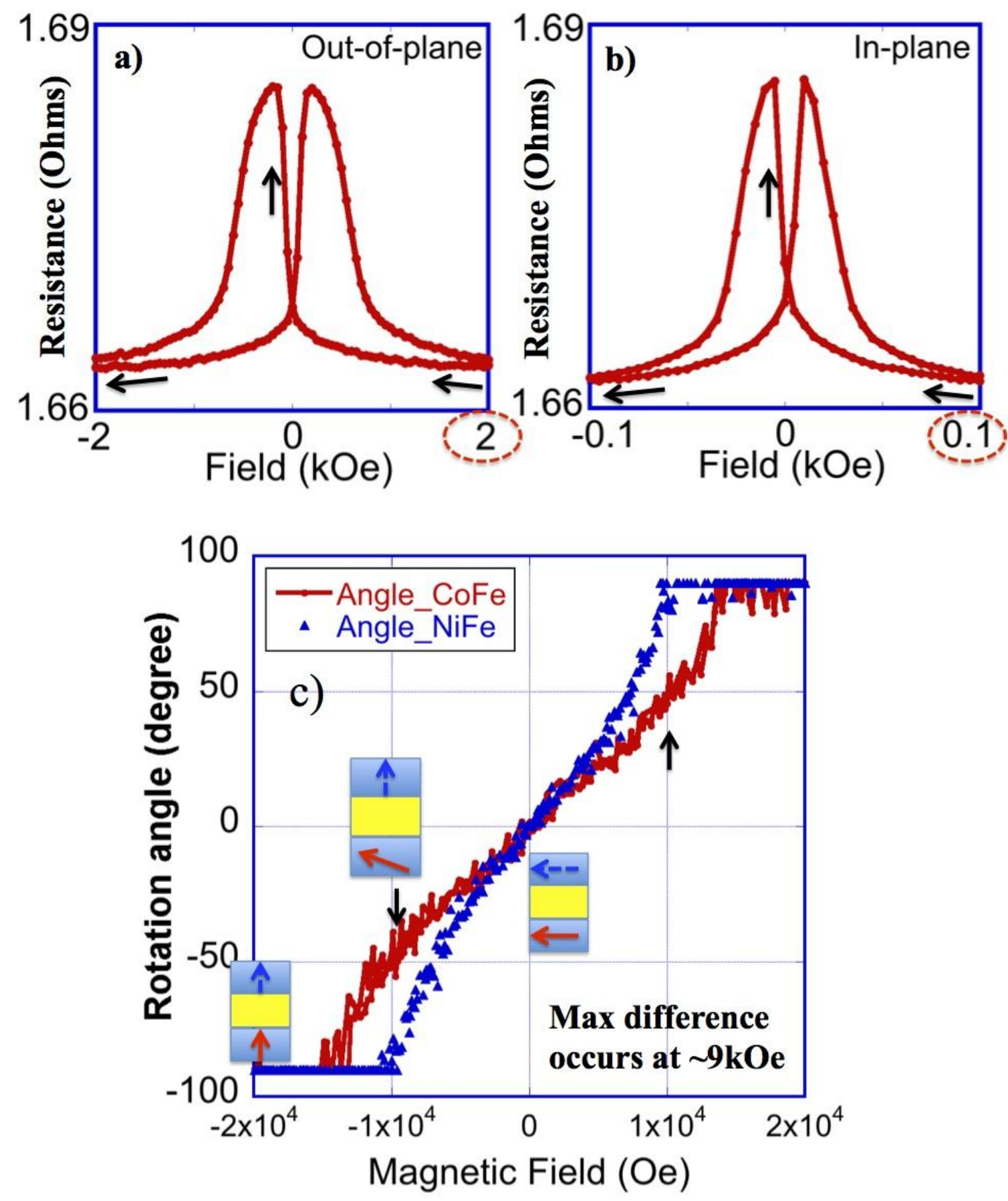

Figure 7.5 (a) Zoom in on the low-field peaks in Figure 7.4. (b) Magnetoresistance curve on the same PSV measured with in-plane field. The arrows indicate the direction of the field sweeping. The dashed circle indicates the scale for the coercive field. (c) 
Rotation angle curves for structure $20 \mathrm{~nm} \mathrm{Ru} / 6.5 \mathrm{~nm} \mathrm{Ni} \mathrm{Fe}_{20} / 5 \mathrm{~nm} \mathrm{Ru}$ (blue triangles) and structure $20 \mathrm{~nm} \mathrm{Ru} / 2.2 \mathrm{~nm} \mathrm{Co} \mathrm{C}_{90} \mathrm{Fe}_{10} / 5 \mathrm{~nm} \mathrm{Ru}$ (red dots). The schematic diagrams show the relative magnetic configuration for the two layers. The black arrows indicate the position for the maximum angle difference between the two layers' magnetization.

\subsection{Spin transfer in nanopillar device}

\subsubsection{Nanostructure of the STT device}

In order to study the spin transfer effect, the film was patterned by photolithography and ion milled to form a round pillar with a cross-section nominal diameter of $500 \mathrm{~nm}$. The details for the device fabrication can be found in Chapter 6. By monitoring the change of thickness and magnetization, the ion milling process could be precisely controlled so that the $\mathrm{Co}_{90} \mathrm{Fe}_{10}$ layer was partially etched (as shown schematically in Figure 7.6(a)). With such a geometry, the $\mathrm{Co}_{90} \mathrm{Fe}_{10}$ fixed layer is relatively insensitive to the spin transfer torques due to its extended volume. Figure 7.6(b) presents a scanning electron microscope image of a $500 \mathrm{~nm}$-diameter size pillar. The final device set-up is shown in the optical microscope image Figure 7.6(c). The differential resistance is measured using a lock-in amplifier circuit in the PPMS system (PPMS 6000, Quantum Design). Positive current is defined so that electrons flow from $\mathrm{Co}_{90} \mathrm{Fe}_{10}$ to $\mathrm{Ni}_{80} \mathrm{Fe}_{20}$. All transport measurements reported in this work were obtained at room temperature. 

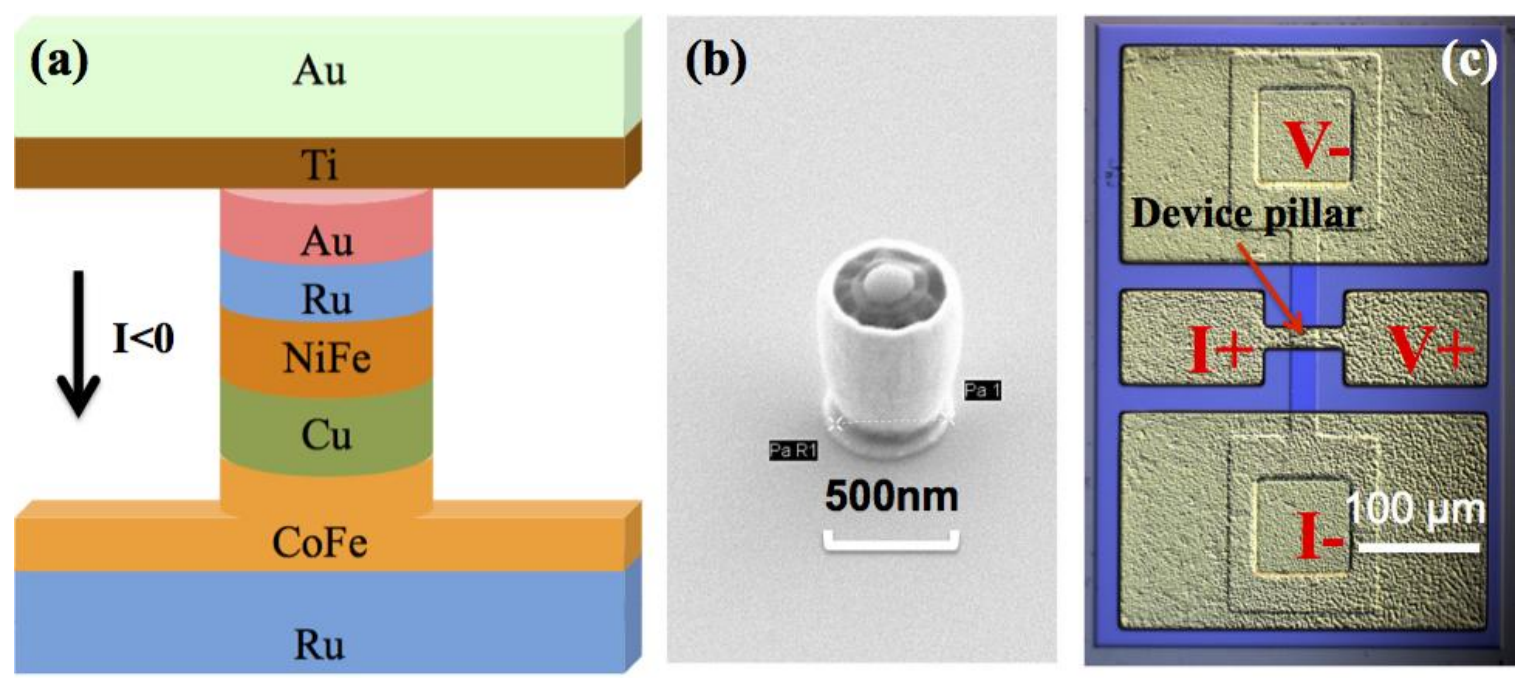

Figure 7.6 (a) Schematic representation of the patterned pseudo-spin-valve sample. The bottom $\mathrm{Co}_{90} \mathrm{Fe}_{10}$ layer is partially patterned. The arrow shows the electron flow direction for negative current. (b) A SEM image of the patterned pillar with a diameter of $500 \mathrm{~nm}$. (c) The optical microscope image of a device with CPW contacts.

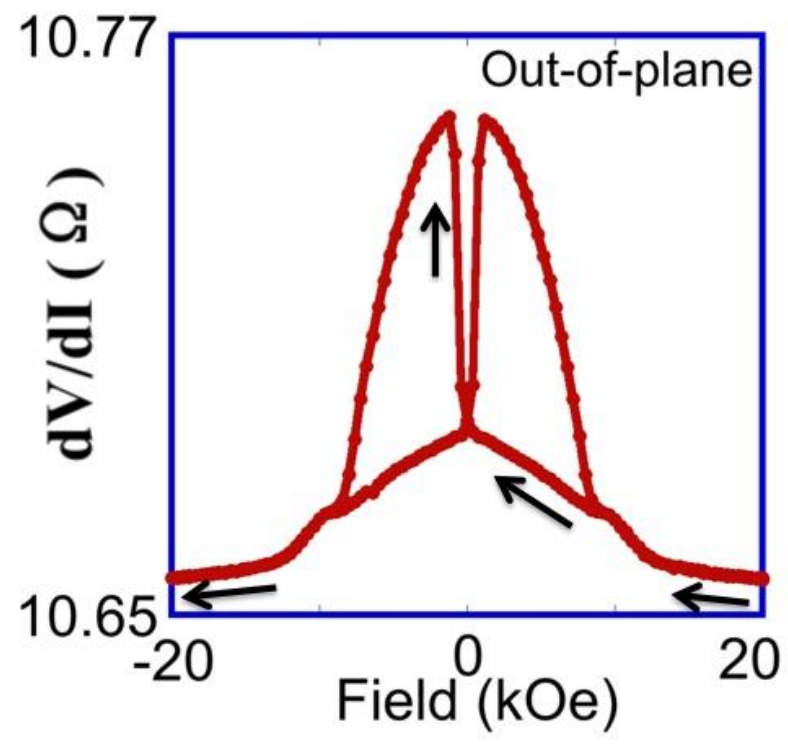

Figure 7.7 Current perpendicular to plane (CPP) MR curve on the patterned sample. The black arrows show the direction of the field sweep. 


\subsubsection{Coercive field of the nanopillar}

The magnetoresistance (MR) of the pseudo spin valve was measured on the full film by current-in-plane method before the patterning process [Figure 7.5 (a)], which is $\sim 1.2 \%$. After CPP patterning, the differential resistance $d V / d I$ versus perpendicular magnetic field $H$ measured on the nano-pillar is plotted in Figure 7.7. The layers switch between antiparallel and parallel alignment with a current perpendicular to the plane (CPP) GMR ratio of $1.0 \%$. The slightly lower GMR ratio after patterning might be due to the lead resistance that was in series with the pillar while it did not contribute to the MR. The lead resistance was mainly coming from the patterned bottom electrod due to its small width $(\sim 20 \mu \mathrm{m})$ and thickness $(\sim 20 \mathrm{~nm})$. The contact resistance was estimated to be around $2.3 \Omega$ based on the original GMR ratio. The estimated value generally agreed with the measured contact resistance of the bottom electrodes $\sim 1.7 \Omega$. The coercive field of both layers has increased significantly over the continuous film values. The $\mathrm{Co}_{90} \mathrm{Fe}_{10}$ reference layer now has a coercive field of $6.56 \mathrm{kOe}$ and the $\mathrm{Ni}_{80} \mathrm{Fe}_{20}$ free layer $1.33 \mathrm{kOe}$. This is in accordance with observations on patterned nano-magnets, where the coercive field increases as it transitions from reversal by means of domain nucleation and domain wall motion in a full film towards coherent rotation in patterned structures [173].

\subsubsection{AMR effect of the nanopillar}

Observing the Figure 7.7, the gradual increase in resistance from $12 \mathrm{kOe}$ to $0 \mathrm{Oe}$ is 
attributed to the anisotropic magnetoresistance (AMR) from the continuous $\mathrm{Co}_{90} \mathrm{Fe}_{10}$ film when the field is reduced. This is confirmed by measuring the magnetoresistance across the two bottom contact pads [between $V$ - and $I$ - as shown in Figure 7.6(c)]. Figure 7.8 shows the results of the sheet resistance $R_{s}$ as a function of the out of plane magnetic field $H$. At high fields, the magnetization of the remaining $\mathrm{Co}_{90} \mathrm{Fe}_{10}$ continuous layer is pulled out of plane; therefore, the magnetization is perpendicular to the current resulting in a low resistance. The zero-field state is a state of high resistance, as the magnetization is parallel or antiparallel to the current due to the in plane uniaxial anisotropy. The field dependence of the resistance shows, to a good approximation, a parabolic behavior as is expected for the AMR when the magnetization rotates coherently [174].

The AMR doesn't affect our study on the spin-transfer effect for three reasons. Firstly, The AMR effect occurs in the bottom layer, while the major spin-transfer effect happens in the $\mathrm{Ni}_{80} \mathrm{Fe}_{20}$ layer. Secondly, we are looking at the differential resistance and the magnetic field is fixed during each current sweeping. Lastly, the GMR is still the dominant effect since the magnitude of GMR is a factor of two larger than that for the AMR effect. 


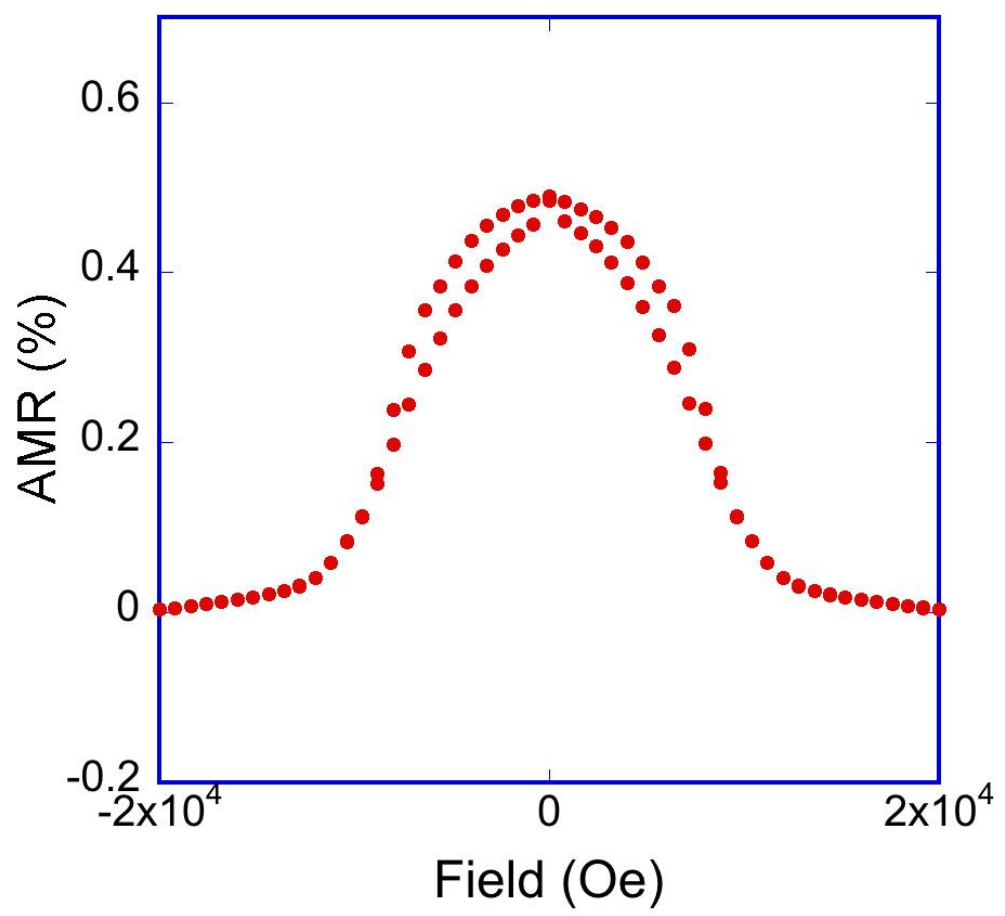

Figure 7.8 Sheet resistance $\mathrm{R}_{\mathrm{S}}$ versus applied magnetic field $\mathrm{H}$ of the continuous $\mathrm{Co}_{90} \mathrm{Fe}_{10}$ layer, measured with the magnetic field perpendicular to the film plane, at room temperature.

\subsubsection{Current induced magnetization reversal in 500nm pillar}

Prior to the magneto-transport measurements, the sample was placed in an out-of-plane magnetic field of $30 \mathrm{kOe}$, and then the field was set to the specific values. In Figure 7.9, we show the variation of the differential resistance $d V / d I$ versus injected direct current at small fields. At $H=0$, starting from the parallel (P) state at zero current, the DC current first decreased toward the negative direction. A jump $\sim 0.1 \Omega$ in the differential 
resistance was observed at $I_{C}^{P-A P}=-48 \mathrm{~mA}$ (current density of $2.45 \times 10^{7} \mathrm{~A} / \mathrm{cm}^{2}$ ) due to the magnetization switching which occurred in the $\mathrm{Ni}_{80} \mathrm{Fe}_{20}$ layer. The curve showed hysteretic behavior because the system remained in the antiparallel (AP) state until the current was swept back to a positive critical current of $I_{C}{ }^{A P-P}=47 \mathrm{~mA}$ (current density of $2.39 \times 10^{7} \mathrm{~A} / \mathrm{cm}^{2}$ ), where the resistance dropped back to the $\mathrm{P}$ state. The corresponding change in the differential resistance was $\sim 95 \mathrm{~m} \Omega$ for both negative and positive switching, which was of the same magnitude of change in resistance shown in the MR minor loop [Figure 7.9 inset]. Therefore, it was certain that the experimental hysteretic curve was caused by spin torque induced magnetization reversal typically observed at zero or low applied magnetic field [4]. The hysteretic reversal here was fundamentally different from the switching by Oersted field, which was self-generated by the current passing through the pillar [175]. The magnitude of the Oersted field was independent of the current direction, which would have resulted in a symmetric $d V / d I$ curve with respect to current. With spin transfer driven magnetization reversal, the effect is not symmetric but depends the direction of the electron flow. Note that the overall parabolic increase in $d V / d I$ can be ascribed to the electron scattering by emissions of phonons and magnons in metallic point contacts [176]. 


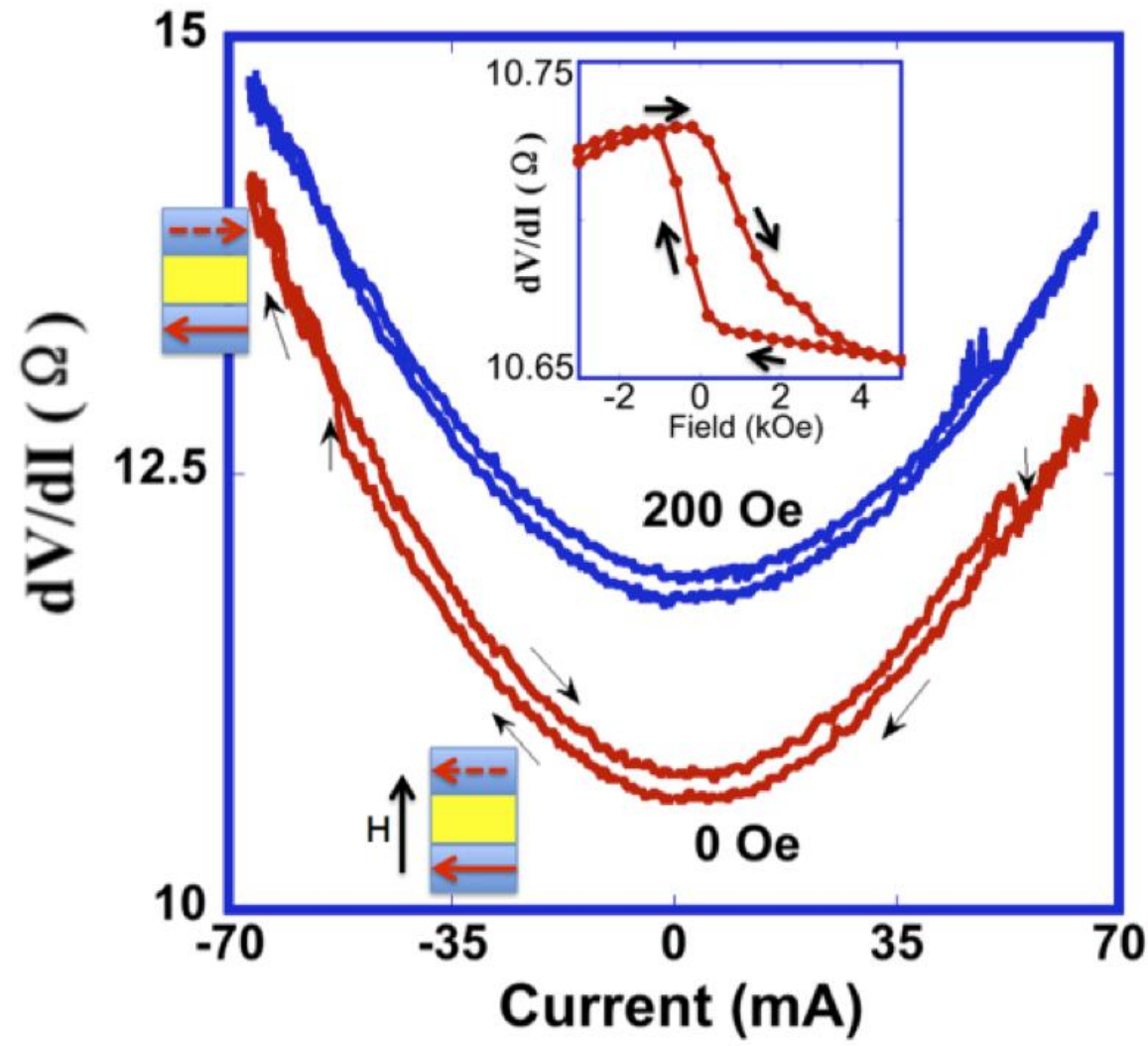

Figure 7.9 Differential resistance versus biased direct current $\left(\mathrm{I}_{\mathrm{B}}\right)$ with out-of-plane magnetic fields of 0 (bottom, red) and 200 Oe (blue). The current sweep starts at $0 \mathrm{~mA}$ and the arrows indicate the direction of the current sweep. The sharp transition happens at $I_{\mathrm{C}}{ }^{\mathrm{P}-\mathrm{AP}}=-48 \mathrm{~mA}\left(\right.$ current density of $\left.2.45 \times 10^{7} \mathrm{~A} / \mathrm{cm}^{2}\right)$ and $I_{\mathrm{C}}{ }^{\mathrm{AP}-\mathrm{P}}=47 \mathrm{~mA}$ (current density of $2.39 \times 10^{7} \mathrm{~A} / \mathrm{cm}^{2}$ ) for $H=0$. At $H=200$ Oe, the switching currents are $I_{\mathrm{C}}{ }^{\text {P-AP }}=$ $-47.2 \mathrm{~mA}$ (current density of $2.40 \times 10^{7} \mathrm{~A} / \mathrm{cm}^{2}$ ) and $I_{\mathrm{C}}{ }^{\text {AP-P }}=46 \mathrm{~mA}$ (current density of $2.35 \times 10^{7} \mathrm{~A} / \mathrm{cm}^{2}$ ). The inset shows the minor MR hysteresis loop for $\mathrm{Ni}_{80} \mathrm{Fe}_{20}$ layer (free layer). The black arrows indicate the direction of the field sweep. 


\subsubsection{Comparison with the Slonczewski model}

I now refer back and make comparison of our experimental results to the Slonczewski model that was outlined in Section 2.4. A series of $d V / d I$ versus $J_{B}$ scans were measured at different fields. The critical current is plotted versus the out-of-plane fields as shown in Figure 7.10. Each symbol in the figure corresponds to a discrete change in the resistance while changing $J_{B}$ at a corresponding field. Under $3.2 \mathrm{kOe}$, we observe hysteretic reversal of magnetization between the AP and P states where the circles (red) correspond to the critical current for AP-P transition $J_{C}{ }^{A P-P}$ and squares (blue) correspond to the critical current for P-AP transition $J_{C}{ }^{P-A P}$.

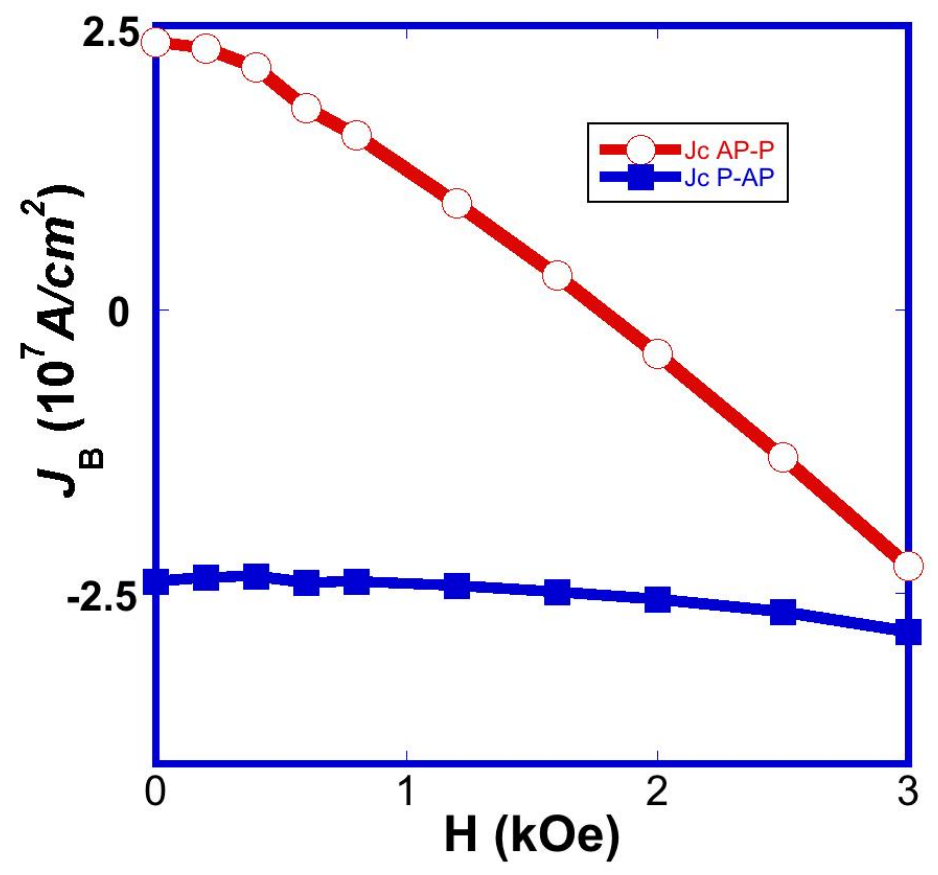

Figure 7.10 Critical current for reversal of magnetization between the AP and P states at different fields, where the circles (red) correspond to the critical current for AP-P transition $J_{C}{ }^{A P-P}$ and squares (blue) correspond to the critical current for P-AP transition $J_{C}{ }^{P-A P}$. 
For $H<3.2 \mathrm{kOe}$, the change of critical current $J_{C}{ }^{A P-P}$ follows a linear $H$ dependence. This agrees with the critical currents equations given by the Slonczewski model $[33,36]$ :

$$
J_{C} \approx \frac{A \alpha M_{s} d}{g(\theta) \mathrm{P}}\left(-H_{K}+4 \pi M_{s}-H_{d i p}-H\right)
$$

where $M_{s}, d$ and $\alpha$ are the saturation magnetization, thickness and Gilbert damping constant for the free layer, respectively. $A$ is a constant coefficient of the order of $10^{11} \mathrm{~mA}$ $\mathrm{Oe}^{-1} \mathrm{emu}^{-1} . P$ is the spin polarization. $g(\theta)$ is a scalar factor depending on $P$ and the relative angle of the reference layer and free layer magnetizations [36]. $H, H_{d i p}$ and $H_{k}$ are the perpendicular field, the dipolar field and the anisotropy field, respectively, while $4 \pi M_{s}$ arises from the demagnetizing field. $J_{\mathrm{C}}{ }^{\mathrm{AP}-\mathrm{P}}$ follows the linear $H$ dependence with a slope $=$ $-3.25 \times 10^{-2} \mathrm{~mA} / \mathrm{Oe}$ and intercepts $J_{B}=0$ near $2 \mathrm{kOe}$, which is roughly the positive coercive field as shown in Figure 7.9(a) inset. By extracting the slope of $J_{\mathrm{C}}{ }^{\text {AP-P }}$ and comparing with the pre-factor in Eq. (7-20), we estimate $\alpha / P=0.42 g(\pi)$. Given that $\alpha=0.009$ and $P=0.4$ [177], then $g(\pi)=0.054$, which is almost an order smaller than the expected range between 0.1 and 0.5 [38]. The difference might be associated with the multiple domains in our large size device. According to the Slonczewski model, Eq. (7-20) only strictly valid for single domain structure at low temperatures [36]. Hence, Eq. (7-20) may not be directly applicable to the possible multi-domain structures in a $500 \mathrm{~nm}$ magnetic nanopillar. 


\subsubsection{DC current induced magnetization oscillation in $500 \mathrm{~nm}$ pillar}

For $H>3.2 \mathrm{kOe}$, discrete switching behavior is no longer observed. Instead, we observe magnetic transitions that are reversible in $I_{B}$ scans and give sharp peaks in $d V / d I$ as shown in Figure 7.11. Regarding the differential resistance measurements $(d V / d I)$, most generally:

$$
\frac{d V}{d I}=\frac{d(I R d . c .)}{d I}=R d . c .+I \frac{d R d . c .}{d I}
$$

Assuming that the system is ohmic such that $d R d_{\text {.c. }} / d I=0$, then $d V / d I=R$ d.c. . However, if there are capacitors or inductors in the circuit, we need to include the second term, $I d R$ d.c. $/ d I$. For the spin torque oscillator, it was equivalent to an inductor-capacitor-resistor (LCR) circuit[178]. Therefore, the second term needed to be included and can add or subtract to $R$ d.c. depending on the sign of the slope $(d R$ d.c. $/ d I)$ and the sign of $I$. In Figure 7.11, the slope of $R$ d.c. versus $I$ (e.g. $d R d . c . / d I$ ) was negative. And the sign of $I$ was also negative giving peaks on $d V / d I$. The amplitude of the peaks was mainly determined by the second term of Eq. (7-21) and reflected the change of $R$ d.c., which was correlated with the spatial trajectories/orbits of the oscillation as explained in the simulation part. The different width of the multiple peaks will be explained later in a micromagnetic simulation part.

Moreover, it is found that these peaks are generated only on the negative currents direction which corresponding to the electron flow direction from $\mathrm{Ni}_{80} \mathrm{Fe}_{20}$ to $\mathrm{Co}_{90} \mathrm{Fe}_{10}$. This feature is also a signature of the spin transfer torque [179]. The change from 
irreversible hysteretic switching to reversible sweeping of the differential curve has been associated in standard nanopillars to the sustained precession of magnetization $[4,180]$. Depending on the external fields, at different current densities, there are different metastable states where the spin transfer torque can balance out the damping leading to such stable precession. Though similar peaks were presented at low temperature $\sim 4 \mathrm{~K}[56$, here all the data presented were taken at room temperature.
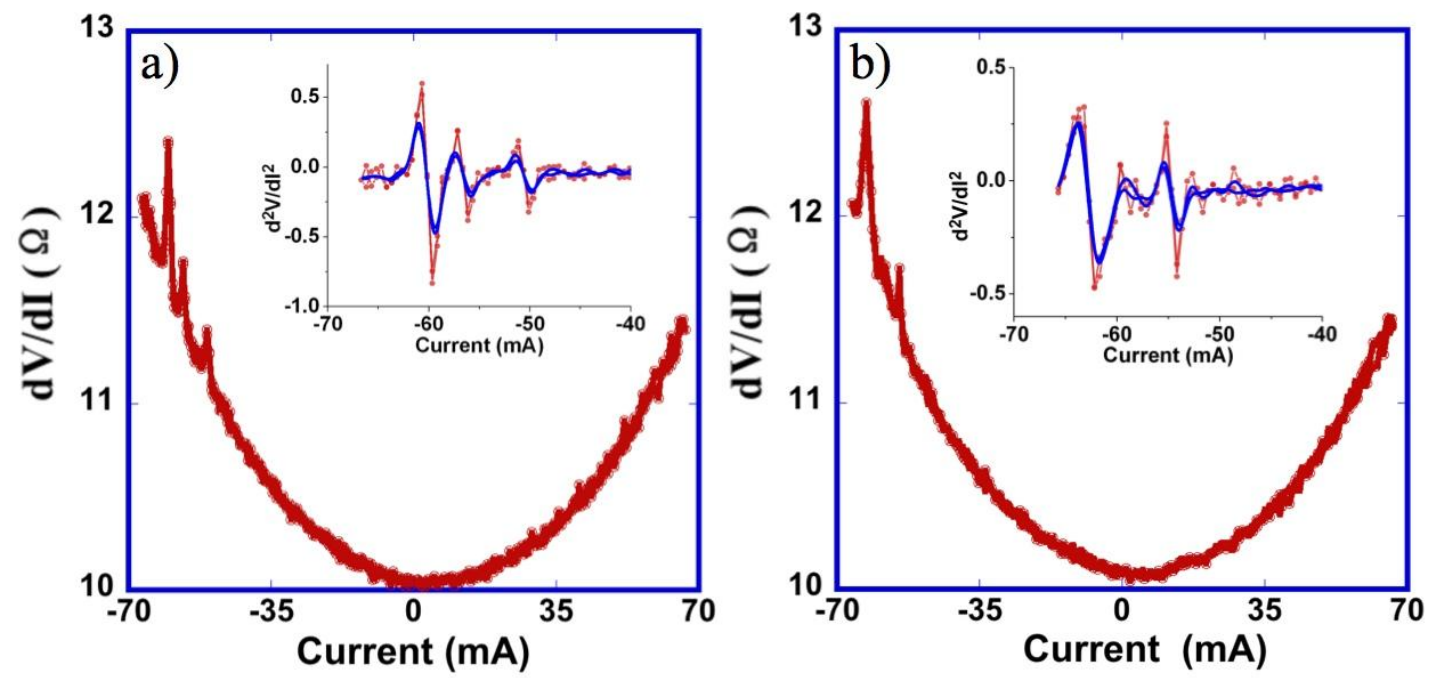

Figure $7.11 d V / d I$ versus $I_{B}$ for (a) $H=3.5 \mathrm{kOe}$ and (b) $H=4.0 \mathrm{kOe}$, the scans are reversible with both current directions. The inset shows the second derivative of $d V / d I$ indicating the relative amplitude, width and position of the peaks. 


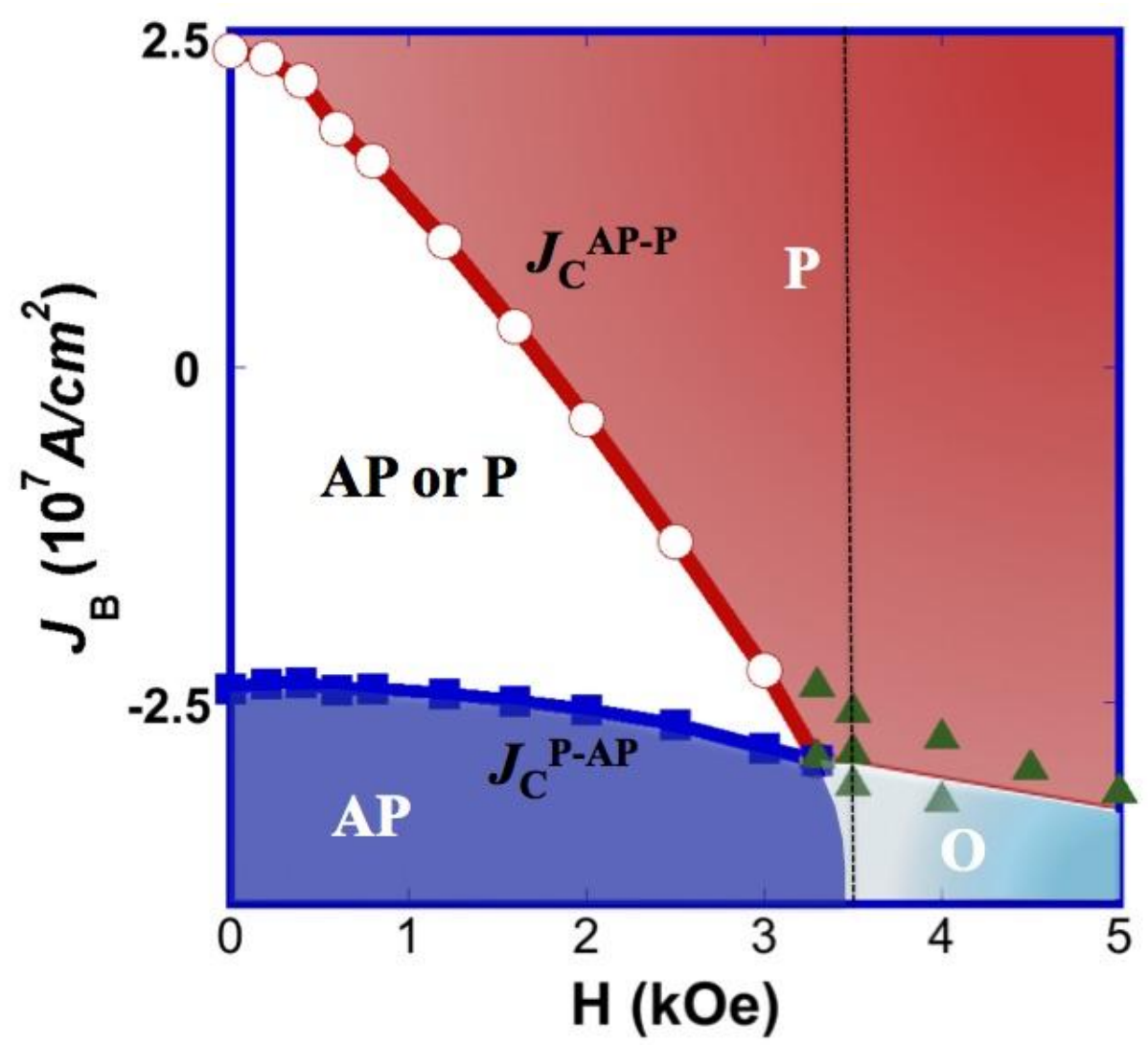

Figure 7.12 Experimentally determined $J_{B}-H$ (Out-of-Plane field) dynamic stability diagram for the patterned sample showing the parallel or antiparallel states where each symbol corresponds to a distinct change in the resistance. Below $3.2 \mathrm{kOe}$, the symbols are from hysteretic switching where the circles (red) are $J_{C}{ }^{A P-P}$ and squares (blue) are $J_{C}$ $P-A P$. For field larger than $3.2 \mathrm{kOe}$, the triangles (green) refer to the peaks on the differential resistance curves. The dashed line corresponds to the reversible peaks at $H=$ 3.5kOe [Figure 7.11(a)].

Starting from parallel alignment of the magnetic layers, a series of $d V / d I$ versus $J_{B}$ scans were measured at different fields to construct the $J_{B^{-}} H$ dynamic stability diagram 
shown in Figure 7.12. Each symbol in the phase diagram corresponds to a discrete change in the resistance while changing $J_{B}$ at a corresponding field. The general features of the dynamic diagram are also seen in $130 \mathrm{~nm} \times 70 \mathrm{~nm} \mathrm{Co} / \mathrm{Cu} / \mathrm{Co}$ devices [4]. Under $3.2 \mathrm{kOe}$, we observe hysteretic reversal of magnetization between the AP and P states where the circles (red) correspond to the critical current for AP-P transition $J_{C}{ }^{A P-P}$ and squares (blue) correspond to the critical current for P-AP transition $J_{C}{ }^{P-A P}$. For $H>3.2 \mathrm{kOe}$ we observe magnetic transitions that are reversible in $I_{B}$ scans and give sharp peaks in $d V / d I$. The reversible change is demonstrated in Figure 7.11(a) with the $d V / d I$ curve recorded at $H=$ $3.5 \mathrm{kOe}$.

In the dynamic stability diagram, the $I_{C}{ }^{P-A P}$ and $I_{C}{ }^{P-A P}$ curves are extrapolated to construct the oscillation regime (the blue regime shown in Figure 7.12). The peaks generally shift to higher current with increasing fields following the trend of the extrapolated curve. Examining Figure 7.11(a), we observe multiple peaks at 3.5 kOe that are corresponding to the marked line in the phase diagram; Furthermore, these peaks have different critical currents and show distinct amplitudes as shown in the second derivative curve Figure 7.11 inset. Given the large size of our device, inhomogeneities, such as multiple domains, exist on the ferromagnetic layers. However, the micromagnetic simulations showed that the multiple domains tended to synchronize with each other under certain currents and led to a harmonic precession as demonstrated in the next section. Based on the simulation, the multiple peaks are likely to be associated with different 
spin-wave modes that are represented by the different precession. A large external field could suppress the number of domains and possibly lead to the single mode precession as shown in the dynamic stability diagram for $H=4.5 \mathrm{kOe}$ and $5 \mathrm{kOe}$, yet, future work needed to be carried out to confirm the spin dynamics experimentally on the frequency domain $[181,182]$.

\subsection{Micromagnetic simulation}

\subsubsection{Micromagnetic simulation of switching in a $500 \mathrm{~nm}$ pillar}

To gain a full understanding of the switching process, micromagnetic simulations were carried out based on the Landau-Lifshitz-Gilbert (LLG) equation including a spin-torque term. Figure 7.13 shows an example of switching from AP to P reproducing the behavior shown in the Figure 7.9 blue curve. For our device, the Oersted field is not strong enough to form a full vortex at the experimental currents used $(\sim 48 \mathrm{~mA})$. The micromagnetic simulation suggests a formation of a full vortex by the Oersted field require a current of at least $\sim 100 \mathrm{~mA}$, the order of which also agrees with reports elsewhere [183]. Instead, a $C$-shape magnetization state tends to form under the Oersted fields, as shown in Figure 7.13. The $C$-state has a majority magnetization pointing along the parallel or anti-parallel direction. Under the action of the spin-transfer torque, the $C$-state rotates toward the opposite direction leading to the switching from antiparallel to parallel. 


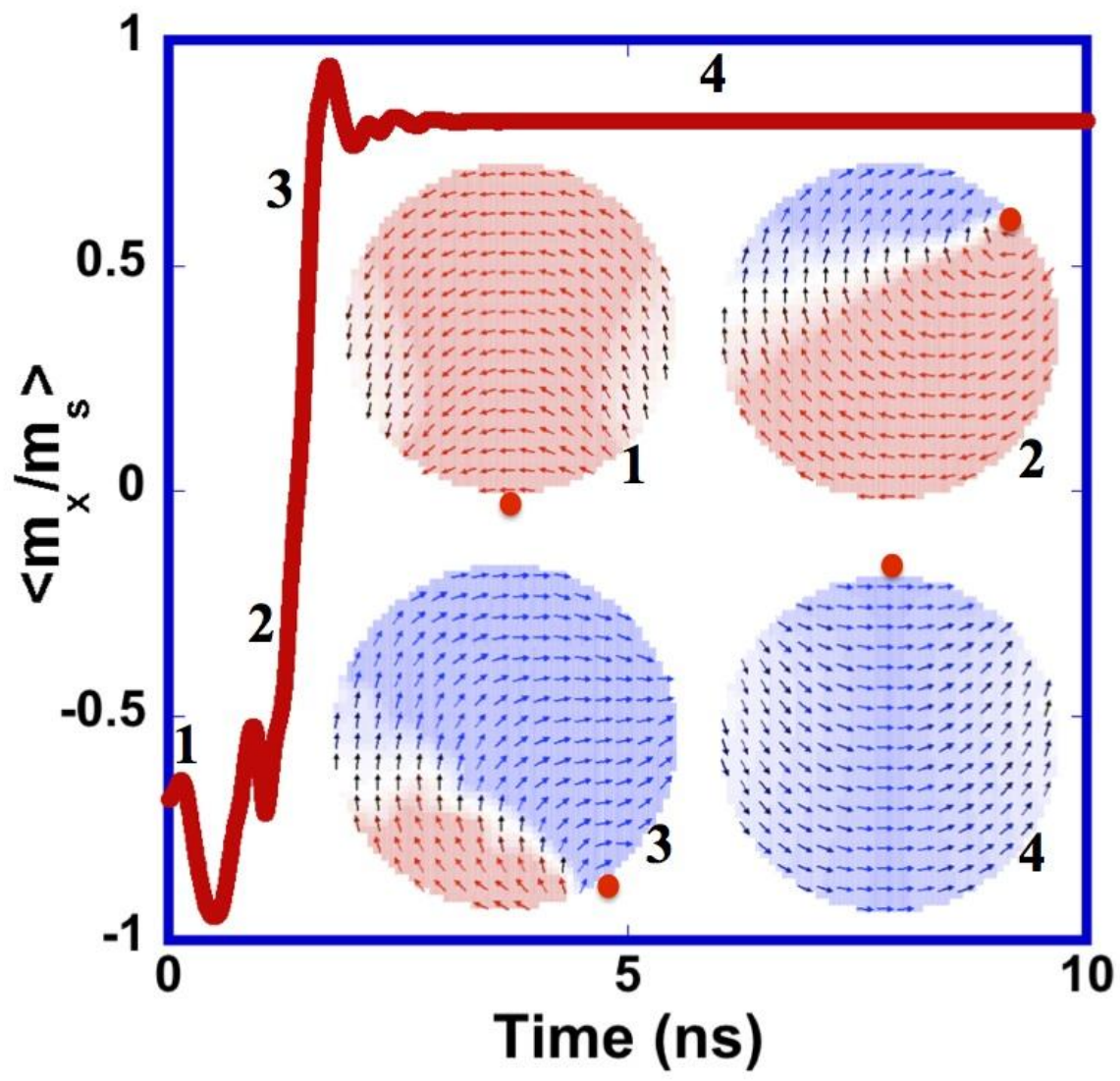

Figure 7.13 Micromagnetic simulation showing the evolution of $\mathrm{m}_{\mathrm{x}}$ component at a current density of $3 \times 10^{7} \mathrm{~A} / \mathrm{cm}^{2}$ with an out-of-plane field $\mathrm{H}=200$ Oe. The flip-over of a $C$-state is demonstrated by the spatial magnetization distribution $1 \rightarrow 2 \rightarrow 3 \rightarrow 4$. The cores are added for illustration purpose.

The critical current density for current-induced magnetization switching in the $500 \mathrm{~nm}$ size pillar was also investigated using numerical simulations. Figure 7.14(a) shows the 
temporal magnetization component evolutions of $\mathrm{m}_{\mathrm{x}}$ with different current densities: $3.0 \times 10^{7} \mathrm{~A} / \mathrm{cm}^{2}$ (red), $2.0 \times 10^{7} \mathrm{~A} / \mathrm{cm}^{2}$ (green) and $1.0 \times 10^{7} \mathrm{~A} / \mathrm{cm}^{2}$ (blue). The results show complete switching from parallel state to antiparallel at higher current density, while no switching is accomplished at lower current density $\sim 1.0 \times 10^{7} \mathrm{~A} / \mathrm{cm}^{2}$. We interpret the results in terms of the energy barrier. The energy barrier between the parallel and antiparallel states mainly comes from the combination of anisotropy energy, dipolar energy and demagnetizing energy. When the current was small (e.g. $1.0 \times 10^{7} \mathrm{~A} / \mathrm{cm}^{2}$ ), the current density was too small to excite the system out of its metastable state. With large enough current density $\left(3.0 \times 10^{7} \mathrm{~A} / \mathrm{cm}^{2}\right)$, the injected spin-polarized current can elevate the total energy of the system and eventually overcome the energy barrier making the system to switch between the parallel and antiparallel states. The simulated evolution of the total energy demonstrated the energy barrier between the P and AP states.
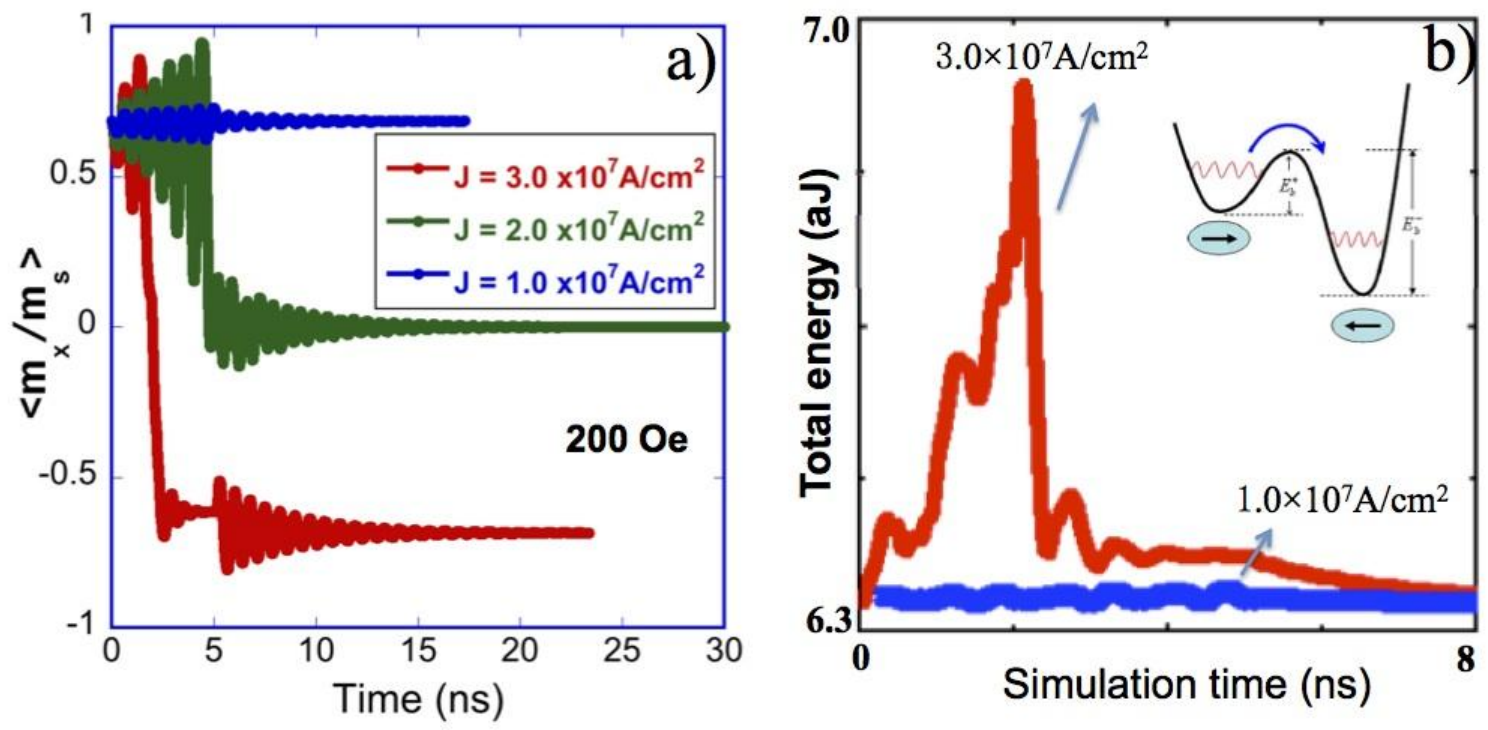
Figure 7.14 (a) The temporal magnetization component evolutions of $m_{x}$ with different current densities: $3.0 \times 10^{7} \mathrm{~A} / \mathrm{cm}^{2}$ (red), $2.0 \times 10^{7} \mathrm{~A} / \mathrm{cm}^{2}$ (green) and $1.0 \times 10^{7} \mathrm{~A} / \mathrm{cm}^{2}$ (blue). (b) The simulated evolution of the total energy for injected current density of $3.0 \times 10^{7} \mathrm{~A} / \mathrm{cm}^{2}$ (red) and $1.0 \times 10^{7} \mathrm{~A} / \mathrm{cm}^{2}$ (blue). The inset shows a schematic diagram of the energy barrier between the parallel and antiparallel state.

\subsubsection{Micromagnetic simulation of oscillations in a $500 \mathrm{~nm}$ pillar}

For $H>3.2 \mathrm{kOe}$, the micromagnetic simulation help us to better understand the intrinsic mechanism for magnetic precession manifested in terms of the multiple peaks in the experimental differential curves. Figure 7.15(a) shows the magneto-static simulation result of magnetization configuration under an out-of-plane field of $3.5 \mathrm{kOe}$. A pseudo hybrid magnetic configuration tends to form under the large field as mentioned in Section 7.2.3. Figure 7.15 (b) shows the magnetization state for the free layer, the $C$-state no longer exist since the external field is dominant than the self-field.

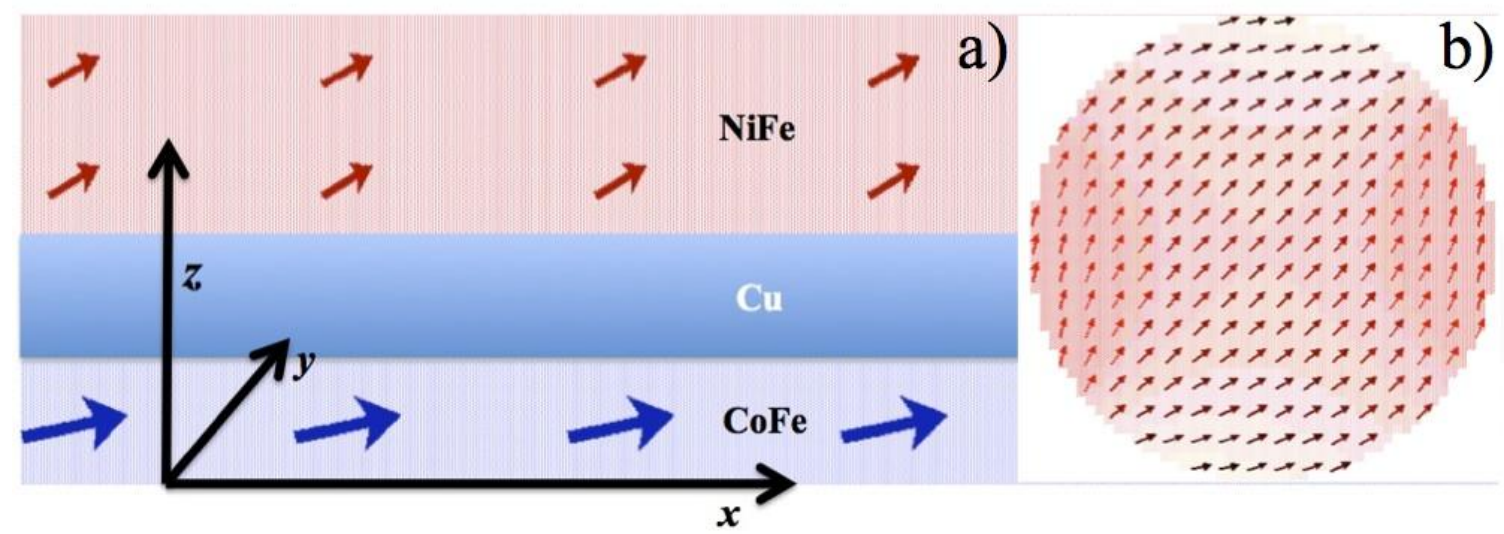

Figure 7.15 The magneto-static simulation results showing the spatial magnetization 
distribution with cross-section view for the whole structure (a) and top view for the free layer (b).
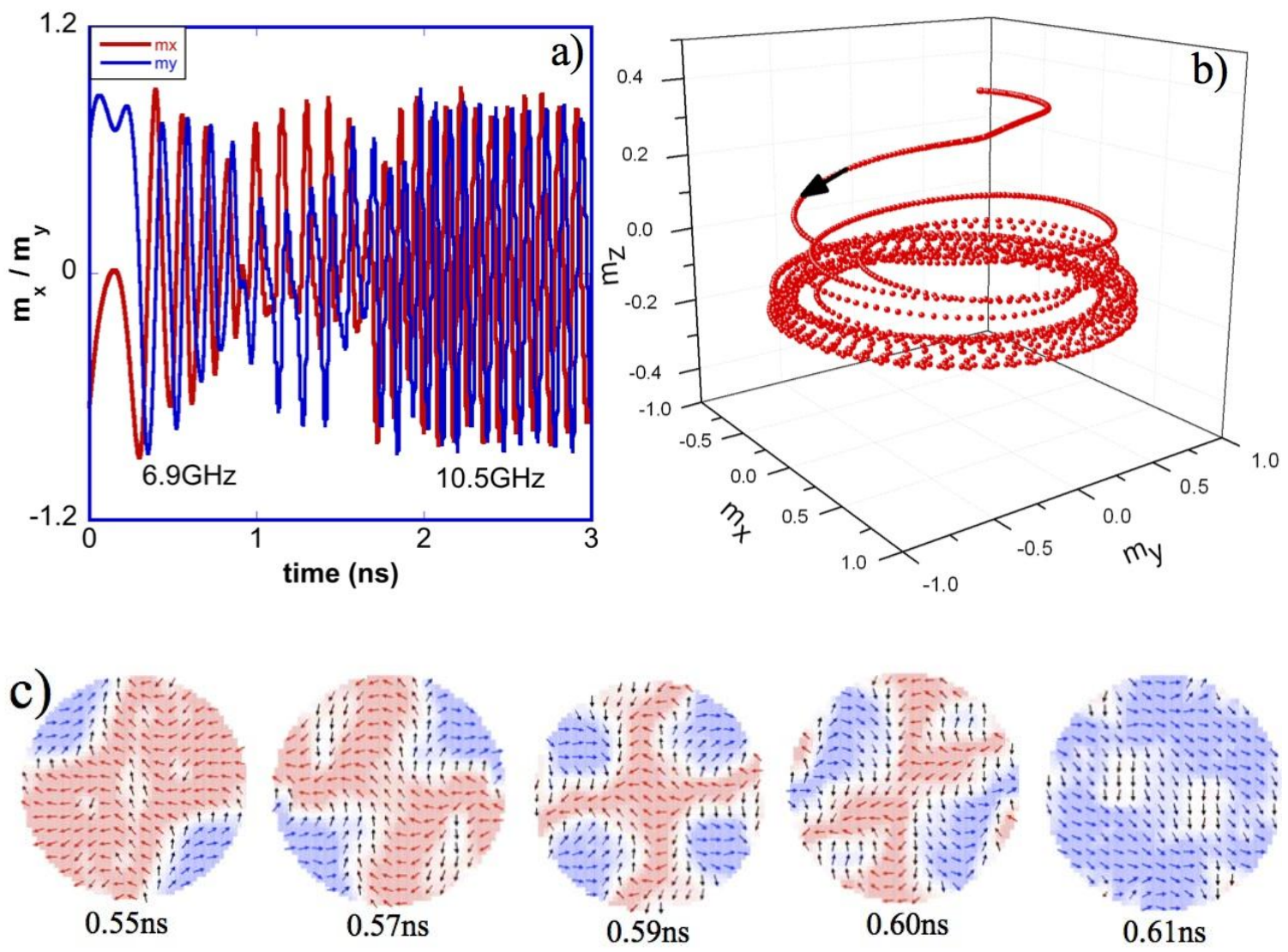

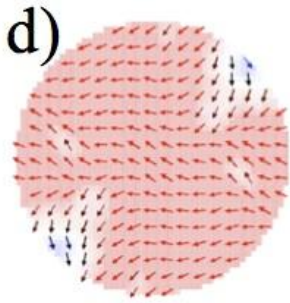

$2.178 \mathrm{~ns}$

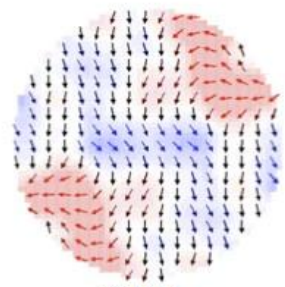

$2.195 \mathrm{~ns}$

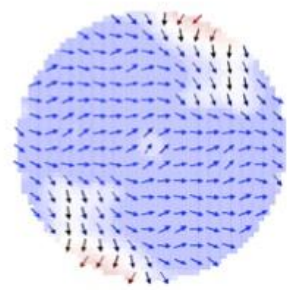

$2.202 \mathrm{~ns}$

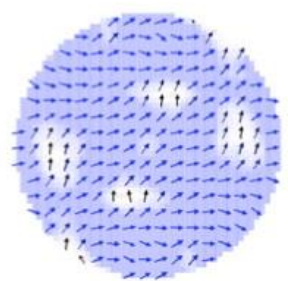

$2.216 \mathrm{~ns}$

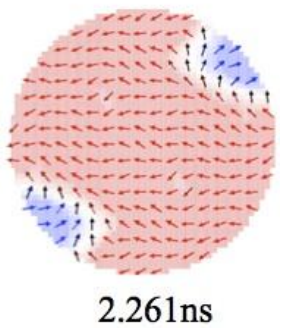

$2.261 \mathrm{~ns}$

Figure 7.16 (a) The temporal magnetization component evolutions of $m_{x}, m_{y}$ under an out-of-plane field of $3.5 \mathrm{kOe}$ and with a constant current density of $3.0 \times 10^{7} \mathrm{~A} / \mathrm{cm}^{2}$. (b) The precession orbits of the free layer magnetization. (c)\&(d) The evolution of the spatial 
magnetization distribution at the corresponding time marked under them. The non-coherent process as shown in (c) features a non-uniform multiple domain oscillation while (d) features a coherent magnetization rotation.

The spin-transfer oscillation was simulated on the structure with an injected current density of $J=3.0 \times 10^{7} \mathrm{~A} / \mathrm{cm}^{2}$, Figure 7.16 (a) shows the evolution of the magnetization components, $m_{x}, m_{y}$. Non-uniform precession presents at the beginning. By looking at the spatial magnetization distribution at the corresponding temporal states[Figure 7.16(c)], it was found that the free layer evolves into a multiple domain states leading to a non-coherent precession. However, these multiple domains eventually lock in with each other and turn into coherent rotation [Figure 7.16(d)]. This is confirmed by looking at the precession orbits as shown in Figure 7.16(b), the moment's orbits is wobbling around the effective field with a relative low frequency of $6.9 \mathrm{GHz}$ at first and gradually reach a stabilized precession with a frequency of $10.5 \mathrm{GHz}$. Based on the spatial orbits as shown in Figure 7.16(b), the different width of the peaks in Figure 7.11(a) can be understood in terms of how quickly the non-coherent precession could be evolved into the coherent oscillation.

Based on the simulation, the multiple peaks in the differential curve is likely to be associated to the different uniform spin-wave modes that are represented by the different precession orbits as shown in Figure 7.17. The orbits shown are numerically calculated 
from the LLGS equation with an out-of-plane applied field of $3.5 \mathrm{kOe}$. At $2.0 \times 10^{7} \mathrm{~A} / \mathrm{cm}^{2}$ (black), the multiple domain non-uniform oscillation is dominant and shows slight variation on the precession orbits. At $3.4 \times 10^{7} \mathrm{~A} / \mathrm{cm}^{2}$ (blue), the non-uniform oscillation quickly evolves into a coherent oscillation featuring with coherent rotation of the magnetization. The coherent oscillation leads to a single orbit precession. The bigger radius of precession orbits at large current densities yields larger GMR change. This also agrees with the experimental curve in terms of that the magnitude of the peak at higher current is bigger than the one with smaller current.

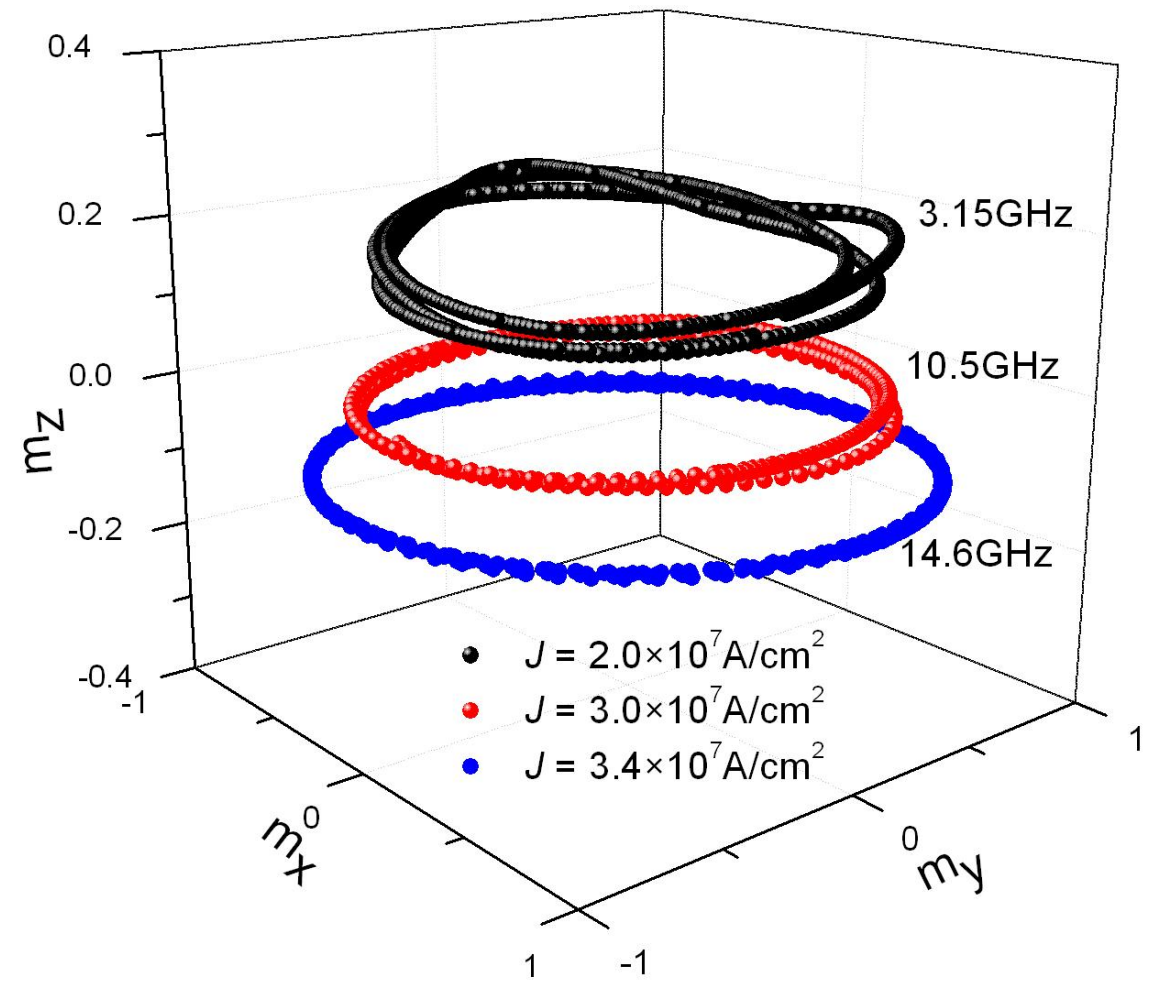

Figure 7.17 At $H=3.5 \mathrm{kOe}$, different current density leads to distinct stabilized precession orbits and frequencies. The main precession frequency $f$ is determined by 
taking the Fourier transforms of $m_{x}$. At $2.0 \times 10^{7} \mathrm{~A} / \mathrm{cm}^{2}$ (black), $3.0 \times 10^{7} \mathrm{~A} / \mathrm{cm}^{2}$ (red) and $3.4 \times 10^{7} \mathrm{~A} / \mathrm{cm}^{2}$ (blue), the simulated oscillation has the frequency of $3.15 \mathrm{GHz}, 10.5 \mathrm{GHz}$ and $14.6 \mathrm{GHz}$ respectively.

It was shown on the dynamic stability diagram (Figure 7.12) that the oscillation modes generally decreased when the field increased (e.g. at $H=3.5 \mathrm{kOe}$, three modes and at $H=$ $4.5 \mathrm{kOe}$ and $5 \mathrm{kOe}$, single mode). To demonstrate the oscillation process at higher external fields, simulation was carried out with an applied field of $H=5 \mathrm{kOe}$ as shown in Figure 7.18. Based on the simulation results, the large external fields can suppress the number of domains and make the oscillation quickly evolved into a coherent oscillation with a major frequency $\sim 22 \mathrm{GHz}$. It is noteworthy to point out that the simulated precession frequency also agrees with the prediction of the Kittel equation[53]. This again suggests that the synchronization of multiple-domain oscillation could be achieved on $500 \mathrm{~nm}$ large sized pillars. 

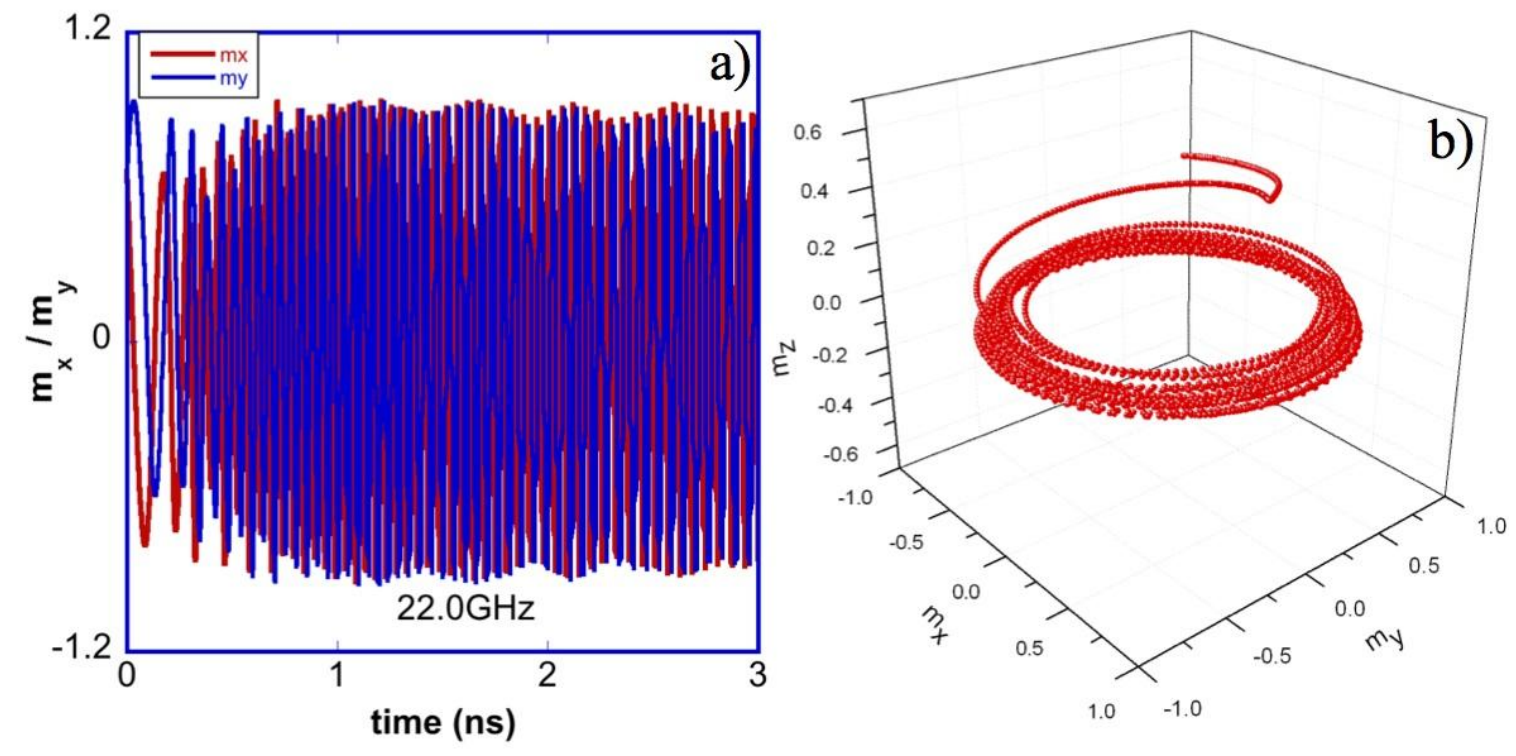

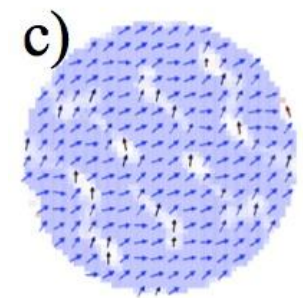

$2.732 \mathrm{~ns}$

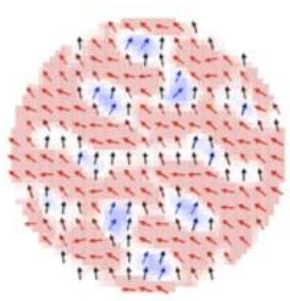

$2.742 \mathrm{~ns}$

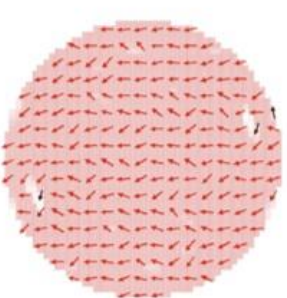

$2.748 \mathrm{~ns}$

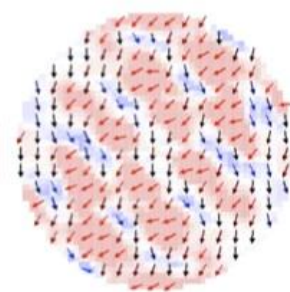

$2.759 \mathrm{~ns}$

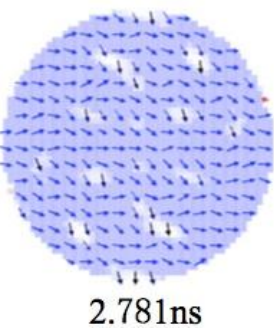

$2.781 \mathrm{~ns}$

Figure 7.18 (a) The temporal magnetization component evolutions of $m_{x}, m_{y}$ under an out-of-plane field of $5.0 \mathrm{kOe}$ and with a constant current density of $3.0 \times 10^{7} \mathrm{~A} / \mathrm{cm}^{2}$.

The precession orbits of the free layer magnetization. (c) The evolution of the spatial magnetization distribution at the corresponding time marked under them.

\subsection{Summary}

In summary, in this chapter I first outlined the reasons why we chose the unique $\mathrm{Co}_{90} \mathrm{Fe}_{10} / \mathrm{Cu} / \mathrm{Ni}_{80} \mathrm{Fe}_{20}$ pseudo spin valve for the study of spin transfer effect. Particularly, a 
hybrid magnetic configuration could be achieved in the structure with perpendicular external fields $>3 \mathrm{kOe}$.

DC current induced magnetization reversal and magnetization oscillation was observed in $500 \mathrm{~nm}$ large size $\mathrm{Co}_{90} \mathrm{Fe}_{10} / \mathrm{Cu} / \mathrm{Ni}_{80} \mathrm{Fe}_{20}$ pillars. A perpendicular external field enhanced the coercive field separation between the reference layer $\left(\mathrm{Co}_{90} \mathrm{Fe}_{10}\right)$ and free layer $\left(\mathrm{Ni}_{80} \mathrm{Fe}_{20}\right)$ in the pseudo spin valve, allowing a large window of external magnetic field for exploring the free-layer reversal. The magnetization precession was manifested in terms of the multiple peaks on the differential resistance curves. Depending on the bias current and applied field, the regions of magnetic switching and magnetization precession on a dynamical stability diagram has been discussed in detail. In contrast to the dynamic diagrams of standard nanopillars, the switching regime of our devices show deviation from the Slonsczewski model, which might be due to the multiple domains of our large size pillar.

Finally, to better interpret the experimental data from the large size pillar. Micromagnetic simulations were carried out. The simulation reproduces well the magnetic reversal process and helps to understand the magnetic switching in terms of the energy barrier. The simulation suggests that the multiple peaks could come from the different spin-wave modes with independent precession orbits. The simulation also indicates that the non-uniform oscillation tends to synchronize with each other and creates large amplitude coherent oscillation at certain current densities. The spin torque oscillation in 
large size devices could lead to potential applications that require enhanced power of the STNOs. 


\section{Chapter 8 Conclusion and future plans}

\subsection{Research Summary}

To summarize, this work explored different magnetic multilayers both from the material perspective and the device aspect. The spin transfer switching and oscillations were demonstrated on $500 \mathrm{~nm}$ large sized pillars. The spin torque oscillation in large size devices could lead to potential applications that require enhanced power of the STNOs.

The major task of this dissertation was to establish the benchmark for spin-torque nano-oscillators fabricated using the BTIBD system and the photolithography techniques in the UVa clean-room facility. To achieve the goal, I studied magnetic multilayer systems deposited using the BTIBD including CFA based multilayers, $\mathrm{L} 1_{0}$ MnAl based multilayers and $\mathrm{NiFe}$ based multilayer system. In the meanwhile, CPP patterning was carried out in clean room facilities to fabricate micro-size and nano-size junctions for the study of spin transfer effect. The most significant spin-transfer effect was observed and discussed in details in the $500 \mathrm{~nm}$ size CPP patterned device made from NiFe based magnetic multilayers.

I evaluated the $\mathrm{Co}_{2} \mathrm{FeAl}$ based magnetic pseudo spin-valve (PSV) structures in terms of the magnetic static and magneto transport properties for their usefulness for STNOs. Starting from the CFA/Cr/CFA trilayers, a very small MR ratio $\sim 0.07 \%$ was observed at room temperature. The small MR ratio is most likely related to the small spin-diffusion 
length for the CFA layer. A small MR ratio will severely affect the output voltage and impede its application on spin-transfer oscillation devices. Optimizations were made on $\mathrm{CFA} / \mathrm{Cu} / \mathrm{CFA}$ and NOL/CFA/Cu/CFA PSVs to improve the MR ratio. Significant enhancement of MR ratio was observed in the NOL/CFA/Cu/CFA PSV due to the specular reflection from the NOL.

I also probed the origin of the magnetoresistance in single layer $\mathrm{L} 1_{0} \mathrm{MnAl}$ by linking the resistivity change to the DW scattering of charge carriers. Quantitative analysis on remanent states' MFM images and the corresponding resistivity confirmed the contribution of DWs to the electric resistivity of MnAl.

The all-perpendicular configuration is considered to be an ideal geometry to produce coherent oscillations without the need for external magnetic field, which makes the integration of large-scale array of STNOs possible [62]. By taking advantage of the strong perpendicular magnetic anisotropy in $\mathrm{L}_{0} \mathrm{MnAl}$, I have successfully fabricated the magnetic hybrid configuration PSV. Magnetic static and magneto transport properties for the structure have been investigated. Low room temperature MR ratio of $\sim 0.05 \%$ was observed on the hybrid PSV possibly due to the small spin-polarization in the thin MnAl films. Further experiments need to be carried out to improve the MR ratio for its application to STNOs.

To obtain a current density on the order of $10^{6} \sim 10^{7} \mathrm{~A} / \mathrm{cm}^{2}$, I have developed the CPP fabrication technique that can be used to fabricate $500 \mathrm{~nm}$ diameters nano-pillars in UVa 
clean-room facility. This process provides cost-effective device preparation and speedy fabrication in large scale. The innovation of introducing an undercut on the photoresist pillar turned out to be very effective in preventing the sidewall re-deposition, hence prevented shunting on the device edge. I also presented the CPP patterning process for magnetic tunnel junction. As introduced in Chapter 2, the MTJ has the major advantage that it provides higher signal to noise ratio due to the significant enhancement of MR by the tunneling effect. Besides, the MTJ can also provide a read-out layer for our pseudo spin-valve devices. Therefore, the successful fabrication and patterning of magnetic tunnel junction films is essential for the STNOs application.

Finally, I demonstrated the magnetization reversal and magnetization oscillation on $500 \mathrm{~nm}$ large sized devices. The magnetization precession was manifested in terms of the multiple peaks on the differential resistance curves. Micromagnetic simulations accurately reproduced the experimental results and provide insight for synchronization of inhomogenieties in large sized pillars.

\subsection{Future plans}

\subsubsection{Preliminary results for all-perpendicular magnetic structure}

In Chapter 5, $\mathrm{L} 1_{0} \mathrm{MnAl}$ was shown to have strong PMA $\left(K_{U^{\sim}} 5.3 \times 10^{6} \mathrm{erg} / \mathrm{cm}^{3}\right)$ due to the uniaxial crystal anisotropy. It had also been reported that PMA $\left(K_{U} \sim 1.6 \times 10^{6} \mathrm{erg} / \mathrm{cm}^{3}\right)$ 
can be achieved on a $\mathrm{Co}_{2} \mathrm{FeAl} / \mathrm{MgO}$ interface when the CFA layer is sufficiently thin (< 2nm) [72]. As a result, all-perpendicular pseudo spin valve structure utilizing $\mathrm{MnAl}$ as the reference layer and $\mathrm{Co}_{2} \mathrm{FeAl}$ as the free layer have been fabricated.

The multilayer films were deposited by BTIBD on a $\mathrm{MgO}$ (001) single crystal substrate with a stacking structure of $\mathrm{Cr}(40 \mathrm{~nm}) / \mathrm{MnAl}(20 \mathrm{~nm}) / \mathrm{Cr}(3 \mathrm{~nm}) / \mathrm{Co}_{2} \mathrm{Fe} \mathrm{Al}(1.7 \mathrm{~nm})$ / $\mathrm{MgO}(2.3 \mathrm{~nm})$. The first three layers were deposited at $200{ }^{\circ} \mathrm{C}$ to improve the chemical ordering of the MnAl layer. The substrate was then cooled down to room temperature and additional layers were deposited, by such a way the interlayer diffusion between $\mathrm{Cr} / \mathrm{Co}_{2} \mathrm{FeAl}$ and $\mathrm{Co}_{2} \mathrm{FeAl} / \mathrm{MgO}$ was decreased.

It is worth to mention that both the $\mathrm{MnAl}$ and $\mathrm{Co}_{2} \mathrm{FeAl}$ layers are epitaxially grown on the $\mathrm{MgO}$ substrate thanks to the lattice match with the $\mathrm{Cr}$ seeding layer. $\mathrm{Cr}$ has a lattice constant $\left(\mathrm{a}_{\mathrm{Cr}}\right) \sim 2.89 \AA$ with a small mismatch to $\mathrm{a}_{\mathrm{MnAl}}(\sim 3 \%)$ and $\mathrm{a}_{\mathrm{Co} 2 \mathrm{FeAl}} / 2(\sim 1 \%)$. Epitaxial growth is important to form an ordered crystal structure, which is necessary for obtaining perpendicular magnetic anisotropy and high magnetization for MnAl as mentioned in Chapter 5.

Figure 8.1 shows the in-plane and out-of-plane magnetic hysteresis loops for the all-perpendicular pseudo-spin-vlave. The out-of-plane M-H loop indicates the successful establishment of PMA for both $\mathrm{MnAl}$ and $\mathrm{Co}_{2} \mathrm{FeAl}$ layers. The sharp magnetization transition near 0 field corresponds to the switch of the $\mathrm{Co}_{2} \mathrm{FeAl}$ layer. The $\mathrm{MnAl}$ reference layer has a much higher coercivity $\sim 0.6 \mathrm{~T}$. Based on the $M_{s}$ for $\mathrm{Co}_{2} \mathrm{FeAl} \sim 1009 \mathrm{emu} / \mathrm{cm}^{3}$ 
and the magnetization change on the $M-H$ loop, an estimated $\sim 0.8 \mathrm{~nm}$ "dead" layer is presented. The "dead" layer is inactive magnetically, and is likely due to the intermixing at the $\mathrm{Co}_{2} \mathrm{FeAl} / \mathrm{Cr}$ interface or the partial oxidation at the $\mathrm{Co}_{2} \mathrm{FeAl} / \mathrm{MgO}$ interface.

The sample was patterned into a Hall bar shape for the study of CIP-GMR. The MR curve indicates a dominant MR contribution from the MnAl layer due to the domain wall scattering. To exclude the DW contribution from MnAl, the sample was CPP-patterned into a $10 \mu \mathrm{m} \times 20 \mu \mathrm{m}$ rectangular cuboid with bottom and top contact pads. The CPP patterned sample shows a relative high resistance $(\sim 26 \Omega)$ due to the presence of an $\mathrm{MgO}$ barrier layer between the top contact pad and the junction itself. No GMR was observed on the CPP-patterned samples.
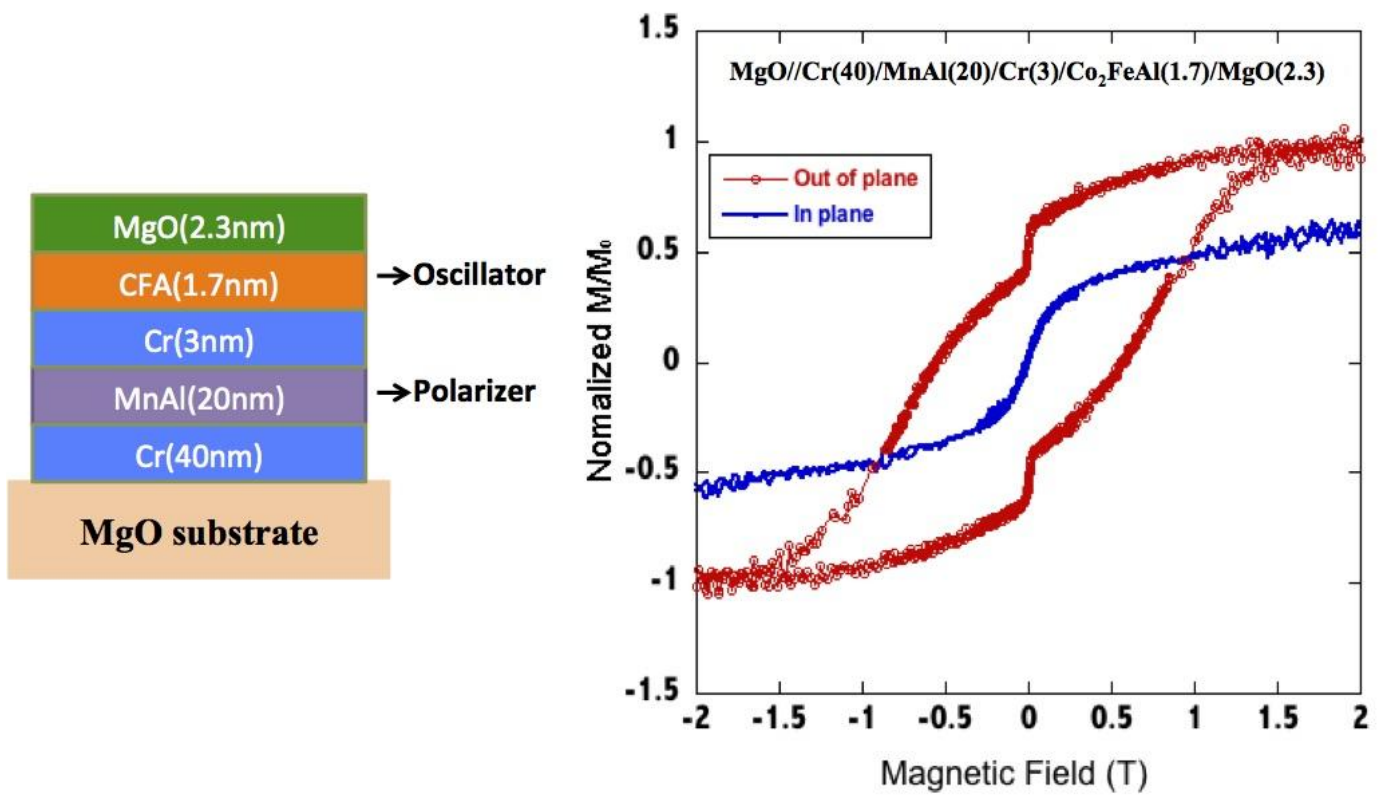

Figure 8.1 Schematic diagram of the all-perpendicular structure and the corresponding In-plane (blue) and out-of-plane (red) $M-H$ loops for the all-perpendicular pseudo spin-valve. The $M-H$ curves are measured at room temperature. 
The MR for the all-perpendicular configuration is expected to be smaller than the MR ratio for the hybrid configuration, e.g. $\sim 0.02 \%$ at room temperature (note: the hybrid structure was discussed in Chapter 5). For one thing, the spin-polarization is likely to be smaller due to the existence of the "dead layer". The "dead" layer suggests that the $\mathrm{Co}_{2} \mathrm{FeAl}$ layer does not have an ideal $\mathrm{B} 2$ structure, which is necessary for obtaining a high spin-polarization. For another, the $\mathrm{MgO}$ layer greatly increases the lead resistance which is in series with the trilayer structure and it does not contribute to the GMR, therefore the effective MR ratio is further decreased. Such a small MR ratio is hard to detect since it is comparable to the noise level. Similar to the $\mathrm{Co}_{2} \mathrm{FeAl}$ based SVs, the MR ratio for the MnAl based multilayers was very small and presented a serious limitation on its applicability for STNOs.

\subsubsection{Future work}

The all-perpendicular configuration is considered to be an ideal geometry to produce

coherent oscillations without the need for external magnetic field, which makes the integration of large-scale array of STNOs possible [62]. However, the all-perpendicular PSV made of $\mathrm{MnAl}$ and $\mathrm{Co}_{2} \mathrm{FeAl}$ electrodes has a MR ratio $<0.02 \%$. Such small a GMR 
will limit the output power of the STNO [4]. To detect such small oscillation signal, an additional read-out layer is required through the magnetic tunneling effect [62]. This additional layer has the drawback of complicating the spin-dependent transport in the device. To improve, future work will be done to fabricate PMA materials based all-perpendicular magnetic tunnel junction structures. MTJ oscillators with large MR ratio ( $>50 \%$ and can be as high as $400 \%[184,185]$ ) at room temperature could provide larger microwave signals than metallic spin-valve oscillators with MR ratios lower than $10 \%$ [58, $61,186]$. The highest output power $(\sim 0.28 \mu \mathrm{W})$ achieved so far was demonstrated in a hybrid MTJ structure with a TMR ratio 100\% [186]. In our system, the TMR ratio for a $\mathrm{Co}_{2} \mathrm{FeAl}$ based in-plane MTJ was characterized to be $\sim 40 \%$. The combination of $\mathrm{Co}_{2} \mathrm{FeAl}$ and NOL is expected to increase the MR ratio according to the discussion in Chapter 4. Further improvement including the utilization of PMA materials such as $\mathrm{Co} / \mathrm{Ni}$ multilayers as a magnetic fixed layer with higher spin-polarization $(P \sim 0.4)$ and reasonable damping $\operatorname{constant}(\alpha=0.025)[171,187]$.

From the device perspective, the spin torque induced switching and oscillation 
features obtained in the $500 \mathrm{~nm}$ pillar strongly indicate that large sized STT devices with reasonable critical current density are achievable. According to our micromagnetic simulation, the multiple domain oscillation on these large devices tends to synchronize into coherent oscillation and is expected to provide higher output power than smaller devices. Future plans will be focused on the experiment confirmation of the synchronization and measurement of the output power using RF techniques. In Ref.[181], the authors demonstrated the in-situ imaging of the magnetization switching in a $100 \times 300 \mathrm{~nm}^{2}$ size pillar by using the time-resolved scanning transmission x-ray microscopy(STXM). The technique was able to detect domain change with both short-time (70 ps) and high-spatial $(25 \mathrm{~nm})$ resolutions. We will consider utilizing such tools to observe and to confirm the synchronization process on our $500 \mathrm{~nm}$ device that had been demonstrated by micromagnetic simulation. Moreover, NiFe pillar devices with different diameters varying from $50 \mathrm{~nm}$ to $500 \mathrm{~nm}$ will be fabricated using electron beam-lithography technique, then the output powers of those different sized STNOs will be characterized using RF techniques and be directly compared. 


\section{References:}

1. S. Wolf et al., Spintronics: a spin-based electronics vision for the future. Science 294, 1488-1495 (2001).

2. J. W. Lu, E. Chen, M. Kabir, M. R. Stan, S. A. Wolf, Spintronics technology: past, present and future. International Materials Reviews 61, 456-472 (2016).

3. Z. Diao et al., Spin transfer switching in dual $\mathrm{MgO}$ magnetic tunnel junctions. Applied Physics Letters 90, 132508 (2007).

4. S. I. Kiselev et al., Microwave oscillations of a nanomagnet driven by a spin-polarized current. Nature 425, 380-383 (2003).

5. W. H. Rippard, M. R. Pufall, S. Kaka, S. E. Russek, T. J. Silva, Direct-Current Induced Dynamics in CoFe/NiFe Point Contacts. Physical Review Letters 92, 027201 (2004).

6. P. Villard et al., A GHz Spintronic-Based RF Oscillator. IEEE Journal of Solid-State Circuits 45, 214-223 (2010).

7. S. E. Russek, W. H. Rippard, T. Cecil, R. Heindl, Handbook of Nanophysics: Functional Nanomaterial ch.38 p.1. Handbook of Nanophysics: Functional Nanomaterial (CRC Press, 2010).

8. M. Kabir, M. Stan, in Spintronics-based Computing, W. Zhao, G. Prenat, Eds. (Springer International Publishing, Cham, 2015), pp. 231-250.

9. Z. Zeng et al., High-Power Coherent Microwave Emission from Magnetic Tunnel Junction Nano-oscillators with Perpendicular Anisotropy. ACS Nano 6, 6115-6121 (2012).

10. M. Nakayama et al., Spin transfer switching in $\mathrm{TbCoFe} / \mathrm{CoFeB} / \mathrm{MgO} / \mathrm{CoFeB} / \mathrm{TbCoFe}$ magnetic tunnel junctions with perpendicular magnetic anisotropy. Journal of Applied Physics 103, 07A710-707A710 (2008).

11. A. Kent, B. Özyilmaz, E. Del Barco, Spin-transfer-induced precessional magnetization reversal. Applied Physics Letters 84, 3897-3899 (2004).

12. K. J. Lee, O. Redon, B. Dieny, Analytical investigation of spin-transfer dynamics using a perpendicular-to-plane polarizer. Applied Physics Letters 86, 022505 
(2005).

13. M. Kabir, M. Stan, in Design Automation Conference (DAC), 2014 51st ACM/EDAC/IEEE. (2014), pp. 1-6.

14. V. S. Pribiag et al., Magnetic vortex oscillator driven by d.c. spin-polarized current. Nature Physics 3, 498-503 (2007).

15. S. M. Mohseni et al., High frequency operation of a spin-torque oscillator at low field. physica status solidi (RRL) - Rapid Research Letters 5, 432-434 (2011).

16. D. B. Gopman et al., Switching field distributions with spin transfer torques in perpendicularly magnetized spin-valve nanopillars. Physical Review B 89, (2014).

17. Z. Zeng et al., Ultralow-current-density and bias-field-free spin-transfer nano-oscillator. Sci Rep 3, 1426 (2013).

18. S. Parkin, Origin of enhanced magnetoresistance of magnetic multilayers: Spin-dependent scattering from magnetic interface states. Physical review letters 71, 1641 (1993).

19. M. N. Baibich et al., Giant magnetoresistance of (001) Fe/(001) Cr magnetic superlattices. Physical review letters 61, 2472 (1988).

20. G. Binasch, P. Grünberg, F. Saurenbach, W. Zinn, Enhanced magnetoresistance in layered magnetic structures with antiferromagnetic interlayer exchange. Physical review B 39, 4828 (1989).

21. R. S. A. o. Sciences, Scientific background on the Nobel Prize in Physics 2007:"The Discovery of Giant Magnetoresistance. Royal Swedish Academy of Sciences (2007).

22. I. Mazin, How to define and calculate the degree of spin polarization in ferromagnets. Physical Review Letters 83, 1427 (1999).

23. P. Grünberg, R. Schreiber, Y. Pang, M. Brodsky, H. Sowers, Layered magnetic structures: evidence for antiferromagnetic coupling of $\mathrm{Fe}$ layers across $\mathrm{Cr}$ interlayers. Physical Review Letters 57, 2442 (1986).

24. B. Dieny et al., Giant magnetoresistive in soft ferromagnetic multilayers. Physical Review B 43, 1297-1300 (1991). 
25. T. Shinjo, Nanomagnetism and spintronics, p.34. (Elsevier, 2013).

26. J. Nogués, I. K. Schuller, Exchange bias. J Magn Magn Mater 192, 203-232 (1999).

27. R. C. O'handley, Modern magnetic materials p.439. (Wiley, 2000).

28. J. Fujikata, K. Hayashi, H. Yamamoto, M. Nakada, Thermal fluctuation aftereffect of exchange coupled films for spin valve devices. Journal of applied physics $\mathbf{8 3}$, 7210-7212 (1998).

29. W. Chen, Structual and material exploration for magnetic tunnel junction, Phd thesis. University of Virginia (2010).

30. M. Julliere, Tunneling between ferromagnetic films. Physics letters A 54, 225-226 (1975).

31. X. Zhang, B.-Z. Li, G. Sun, F.-C. Pu, Spin-polarized tunneling and magnetoresistance in ferromagnet/insulator(semiconductor) single and double tunnel junctions subjected to an electric field. Physical Review B 56, 5484-5488 (1997).

32. L. Berger, Emission of spin waves by a magnetic multilayer traversed by a current. Physical Review B 54, 9353 (1996).

33. J. C. Slonczewski, Current-driven excitation of magnetic multilayers. J Magn Magn Mater 159, L1-L7 (1996).

34. L. Berger, Emission of spin waves by a magnetic multilayer traversed by a current. Physical Review B 54, 9353-9358 (1996).

35. F. J. Albert, The Fabrication and Measurement of Current Perpendicular to the Plane Magnetic Nanostructures for the Study of the Spin Transfer Effect p.92-100. Cornell Univ., (2003).

36. J. C. Slonczewski, Currents and torques in metallic magnetic multilayers. J Magn Magn Mater 247, 324-338 (2002).

37. M. Donahue, D. Porter, Oommf user's guide, version 1.0, interagency report nistir 6376. National Institute of Standards and Technology, (1999).

38. S. Mangin et al., Current-induced magnetization reversal in nanopillars with perpendicular anisotropy. Nat Mater 5, 210-215 (2006). 
39. R. Sbiaa et al., Reduction of switching current by spin transfer torque effect in perpendicular anisotropy magnetoresistive devices (invited). Journal of Applied Physics 109, 07 C707 (2011).

40. E. Myers, D. Ralph, J. Katine, R. Louie, R. Buhrman, Current-induced switching of domains in magnetic multilayer devices. Science 285, 867-870 (1999).

41. K. Yagami, A. A. Tulapurkar, A. Fukushima, Y. Suzuki, Low-current spin-transfer switching and its thermal durability in a low-saturation-magnetization nanomagnet. Applied Physics Letters 85, 5634-5636 (2004).

42. G. D. Fuchs et al., Adjustable spin torque in magnetic tunnel junctions with two fixed layers. Applied Physics Letters 86, 152509 (2005).

43. T. Ochiai et al., Distinctive current-induced magnetization switching in a current-perpendicular-to-plane giant-magnetoresistance nanopillar with a synthetic antiferromagnet free layer. Applied Physics Letters 86, 242506 (2005).

44. Y. Jiang et al., Effective Reduction of Critical Current for Current-Induced Magnetization Switching by a Ru Layer Insertion in an Exchange-Biased Spin Valve. Physical Review Letters 92, 167204 (2004).

45. X. Guo et al., Reduction of magnetic damping constant of FeCo films by rare-earth Gd doping. Applied Physics Letters 105, 072411 (2014).

46. O. Ozatay et al., Spin transfer by nonuniform current injection into a nanomagnet. Applied Physics Letters 88, 202502 (2006).

47. M. C. Wu et al., Reduction in critical current of current induced switching in an inhomogeneous nanomagnet. Applied Physics Letters 94, 122511 (2009).

48. W. J. Gallagher, S. S. Parkin, Development of the magnetic tunnel junction MRAM at IBM: from first junctions to a 16-Mb MRAM demonstrator chip. IBM Journal of Research and Development 50, 5-23 (2006).

49. X. Wang, Y. Chen, H. Li, D. Dimitrov, H. Liu, Spin torque random access memory down to $22 \mathrm{~nm}$ technology. IEEE transactions on magnetics 44, 2479-2482 (2008).

50. X. Zhu, J.-G. Zhu, Spin torque and field-driven perpendicular MRAM designs scalable to multi-Gb/chip capacity. IEEE transactions on magnetics $\mathbf{4 2}$, 2739-2741 (2006). 
51. S. A. Wolf, A. Y. Chtchelkanova, D. M. Treger, Spintronics-A retrospective and perspective. IBM journal of research and development 50, 101-110 (2006).

52. M. Tsoi et al., Excitation of a magnetic multilayer by an electric current. Physical Review Letters 80, 4281 (1998).

53. C. Kittel, On the Theory of Ferromagnetic Resonance Absorption. Physical Review 73, 155-161 (1948).

54. K.-J. Lee, A. Deac, O. Redon, J.-P. Nozieres, B. Dieny, Excitations of incoherent spin-waves due to spin-transfer torque. Nature Materials 3, 877-881 (2004).

55. D. Houssameddine et al., Spin-torque oscillator using a perpendicular polarizer and a planar free layer. Nat Mater 6, 441-447 (2007).

56. W. H. Rippard, M. R. Pufall, T. J. Silva, Quantitative studies of spin-momentum-transfer-induced excitations in $\mathrm{Co} / \mathrm{Cu}$ multilayer films using point-contact spectroscopy. Applied Physics Letters 82, 1260-1262 (2003).

57. F. B. Mancoff, N. D. Rizzo, B. N. Engel, S. Tehrani, Phase-locking in double-point-contact spin-transfer devices. Nature 437, 393-395 (2005).

58. A. M. Deac et al., Bias-driven high-power microwave emission from MgO-based tunnel magnetoresistance devices. Nature Physics 4, 803-809 (2008).

59. S. Kaka et al., Mutual phase-locking of microwave spin torque nano-oscillators. Nature 437, 389-392 (2005).

60. T. Shinjo, Nanomagnetism and spintronics. (Elsevier, 2013), pp. 132.

61. D. Houssameddine et al., Spin transfer induced coherent microwave emission with large power from nanoscale $\mathrm{MgO}$ tunnel junctions. Applied Physics Letters 93, 2505 (2008).

62. M. Kabir, M. Stan, Computing with Hybrid CMOS/STO Circuits. 1-6 (2014).

63. W. H. Meiklejohn, C. P. Bean, New magnetic anisotropy. Physical review 102, 1413 (1956).

64. S. Ikeda et al., A perpendicular-anisotropy $\mathrm{CoFeB}-\mathrm{MgO}$ magnetic tunnel junction. Nature materials 9, 721-724 (2010).

65. B. D. Cullity, C. D. Graham, Introduction to magnetic materials, p.199-202. (John Wiley \& Sons, 2011). 
66. W. Sucksmith, J. E. Thompson, in Proceedings of the Royal Society of London A: Mathematical, Physical and Engineering Sciences. (The Royal Society, 1954), vol. 225, pp. 362-375.

67. C. Chappert, P. Bruno, Magnetic anisotropy in metallic ultrathin films and related experiments on cobalt films. Journal of applied physics 64, 5736-5741 (1988).

68. B. D. Cullity, C. D. Graham, Introduction to magnetic materials , p.52-55. (John Wiley \& Sons, 2011).

69. M. Johnson, P. Bloemen, F. Den Broeder, J. De Vries, Magnetic anisotropy in metallic multilayers. Reports on Progress in Physics (1996), vol. 59, pp. 1409.

70. Z. Wen, H. Sukegawa, S. Mitani, K. Inomata, Perpendicular magnetization of $\mathrm{Co} 2 \mathrm{FeAl}$ full-Heusler alloy films induced by $\mathrm{MgO}$ interface. Applied Physics Letters 98, 2507 (2011).

71. L. Néel et al., Magnetic surface anisotropy and superlattice formation by orientation. Anisotropie magnétique superficielle et surstructures d'orientation p. 225. Journal de Physique et le Radium 15, (1954).

72. Y. Cui et al., Interfacial perpendicular magnetic anisotropy and damping parameter in ultra thin Co2FeAl films. Applied Physics Letters 102, 162403 (2013).

73. L. Luo et al., Magneto-transport and domain wall scattering in epitaxy L10 MnAl thin film. Journal of Applied Physics 119, 103902 (2016).

74. B. D. Cullity, C. D. Graham, Introduction to magnetic materials, p.225. (John Wiley \& Sons, 2011).

75. S. Mizukami et al., Low damping constant for Co2FeAl Heusler alloy films and its correlation with density of states. Journal of Applied Physics 105, 07 D306 (2009).

76. M. I. Katsnelson, V. Y. Irkhin, L. Chioncel, A. I. Lichtenstein, R. A. de Groot, Half-metallic ferromagnets: From band structure to many-body effects. Reviews of Modern Physics 80, 315-378 (2008).

77. Y. Miura, K. Nagao, M. Shirai, Atomic disorder effects on half-metallicity of the full-Heusler alloys Co2Cr1-xFexAl: A first-principles study. Physical Review B 69, 144413 (2004).

78. M. Sargolzaei et al., Spin and orbital magnetism in full Heusler alloys: A density 
functional theory study of Co2 YZ(Y=Mn or Fe;Z=Al, Si, Ga, Ge). Physical Review $B$ 74, 224410 (2006).

79. T. Oikawa et al., Microstructure and magnetic properties of $\mathrm{CoPtCr}-\mathrm{SiO}<\mathrm{sub}>2</ \mathrm{sub}>$ perpendicular recording media. Magnetics, IEEE Transactions on 38, 1976-1978 (2002).

80. S. Mangin et al., Current-induced magnetization reversal in nanopillars with perpendicular anisotropy. Nature Materials 5, 210-215 (2006).

81. S. Mizukami et al., Fast magnetization precession observed in L1[sub 0]-FePt epitaxial thin film. Applied Physics Letters 98, 052501 (2011).

82. M. Hosoda et al., Fabrication of L10-MnAl perpendicularly magnetized thin films for perpendicular magnetic tunnel junctions. Journal of Applied Physics 111, $07 \mathrm{~A} 324$ (2012).

83. A. J. J. Koch, P. Hokkeling, M. G. v. d. Steeg, K. J. de Vos, New Material for Permanent Magnets on a Base of Mn and Al. Journal of Applied Physics 31, S75-S77 (1960).

84. H. Saruyama, M. Oogane, Y. Kurimoto, H. Naganuma, Y. Ando, Fabrication of L10-Ordered MnAl Films for Observation of Tunnel Magnetoresistance Effect. Japanese Journal of Applied Physics 52, 063003 (2013).

85. D. C. Ralph et al., Spin-transfer torque in nanoscale magnetic devices. Philosophical Transactions of the Royal Society of London A: Mathematical, Physical and Engineering Sciences 369, 3617-3630 (2011).

86. S. S. P. Parkin, M. Hayashi, L. Thomas, Magnetic Domain-Wall Racetrack Memory. Science 320, 190-194 (2008).

87. P. M. Levy, S. Zhang, Resistivity due to Domain Wall Scattering. Physical Review Letters 79, 5110-5113 (1997).

88. M. Viret et al., Spin scattering in ferromagnetic thin films. Physical Review B 53, 8464-8468 (1996).

89. J. F. Gregg et al., Giant Magnetoresistive Effects in a Single Element Magnetic Thin Film. Physical Review Letters 77, 1580-1583 (1996).

90. U. Rüdiger, J. Yu, L. Thomas, S. S. P. Parkin, A. D. Kent, Magnetoresistance, 
micromagnetism, and domain-wall scattering in epitaxial hcp Co films. Physical Review B 59, 11914-11918 (1999).

91. J. Yu et al., Magnetotransport and magnetic properties of molecular-beam epitaxy L1[sub 0] FePt thin films. Journal of Applied Physics 87, 6854 (2000).

92. M. Viret et al., Anisotropy of Domain Wall Resistance. Physical Review Letters 85, 3962-3965 (2000).

93. A. Sakuma, Electronic Structure and Magnetocrystalline Anisotropy Energy of MnAl. Journal of the Physical Society of Japan 63, 1422-1428 (1994).

94. H. Kōno, On the Ferromagnetic Phase in Manganese-Aluminum System. Journal of the Physical Society of Japan 13, 1444-1451 (1958).

95. Y. Cui, Material Exploration for spin transfer torque-magnetic random access memory, PhD thesis. University of Virginia (2013).

96. X. W. Zhou, H. N. G. Wadley, Mechanisms of inert gas impact induced interlayer mixing in metal multilayers grown by sputter deposition. Journal of Applied Physics 90, 3359-3366 (2001).

97. J. Quan, Low Energy Ion Beam Assisted Growth of Metal Multilayers, Phd Thesis. (2007).

98. T. L. Hylton et al., Thin film processing by biased target ion beam deposition. IEEE Transactions on Magnetics 36, 2966-2971 (2000).

99. D. M. C. Nicholson et al., Magnetic structure of the spin valve interface. Journal of Applied Physics 76, 6805-6807 (1994).

100. H. Wadley, X. Zhou, J. Quan, Biased Target Ion Beam Deposition of Spin-Valves in Proceedings of the Non-Volatile Memory Technology Symposium. San Diego, 12-11 (2003).

101. J. J. Quan, S. A. Wolf, H. N. G. Wadley, Low energy ion beam assisted deposition of a spin valve. Journal of Applied Physics 101, 074302 (2007).

102. H. Todokoro, M. Ezumi. (Google Patents, 1999).

103. Generating a SEM image. http://www.ammrf.org.au/.

104. B. Tompkins. (Digital Instruments Veeco Metrology Group, Santa Barbara, 1999).

105. J. Z. Sun, Spin angular momentum transfer in current-perpendicular nanomagnetic 
junctions. IBM journal of research and development 50, 81-100 (2006).

106. G. Bertotti et al., Magnetization switching and microwave oscillations in nanomagnets driven by spin-polarized currents. Physical review letters 94, 127206 (2005).

107. X. Xu, J. Zhang, L. Sha, D. Zhang, Y. Jiang, A clear oscillation of the interlayer exchange coupling in $\mathrm{Co} 2 \mathrm{Fe} \mathrm{Al} / \mathrm{Cr} / \mathrm{Co} 2 \mathrm{FeAl}$ structure with $\mathrm{MgO}$ capping layer. Journal of Applied Physics 112, 073904 (2012).

108. S. O. Demokritov, A. B. Drovosekov, D. I. Kholin, N. M. Kreines, Temperature dependence of interlayer coupling in a $\mathrm{Fe} / \mathrm{Cr} / \mathrm{Fe}$ wedge sample. MOKE and MBLS studies. J Magn Magn Mater 258-259, 391-393 (2003).

109. E. E. Fullerton, K. T. Riggs, C. H. Sowers, S. D. Bader, A. Berger, Suppression of Biquadratic Coupling in Fe $\$ / \$ C r(001)$ Superlattices below the Nl'eel Transition of Cr. Physical Review Letters 75, 330-333 (1995).

110. E. E. Fullerton, S. D. Bader, Temperature-dependent biquadratic coupling in antiferromagnetically coupled $\mathrm{Fe} / \mathrm{FeSi}$ multilayers. Physical Review $B$ 53, 5112-5115 (1996)

111. M. Schäfer et al., Investigation of $90^{\circ}$ coupling in $\mathrm{Fe} / \mathrm{Ag} / \mathrm{Fe}$ structures: "Loose spins" and fluctuation mechanism. Journal of Applied Physics 77, 6432-6438 (1995).

112. H. Wang et al., Oscillatory interlayer exchange coupling in epitaxial Co2MnSi/Cr/Co2MnSi trilayers. Applied physics letters 90, 2510 (2007).

113. H. Wang et al., Numerical simulation of magnetization process in epitaxial $\mathrm{Co} 2 \mathrm{MnSi} / \mathrm{Cr} / \mathrm{Co} 2 \mathrm{MnSi}$ trilayers with oscillatory interlayer coupling. Journal of applied physics 101, (2007).

114. F. J. Albert, N. C. Emley, E. B. Myers, D. C. Ralph, R. A. Buhrman, Quantitative Study of Magnetization Reversal by Spin-Polarized Current in Magnetic Multilayer Nanopillars. Physical Review Letters 89, 226802 (2002).

115. W. Wang et al., Thickness-dependent structural, magnetic and transport properties of epitaxial Co2FeAl Heusler alloy thin films. ArXiv e-prints. 2012.

116. Z. Wen et al., A 4-Fold-Symmetry Hexagonal Ruthenium for Magnetic 
Heterostructures Exhibiting Enhanced Perpendicular Magnetic Anisotropy and Tunnel Magnetoresistance. Advanced Materials 26, 6483-6490 (2014).

117. Y. C. Wang et al., High-coercivity Co-ferrite thin films on (100)-SiO2 substrate. Applied Physics Letters 84, 2596-2598 (2004).

118. W. Wang, H. Sukegawa, R. Shan, S. Mitani, K. Inomata, Giant tunneling magnetoresistance up to $330 \%$ at room temperature in sputter deposited $\mathrm{Co} 2 \mathrm{Fe} \mathrm{Al} / \mathrm{MgO} / \mathrm{CoFe}$ magnetic tunnel junctions. Applied Physics Letters 95, 182502 (2009).

119. W. Wang, H. Sukegawa, K. Inomata, Temperature dependence of tunneling magnetoresistance in epitaxial magnetic tunnel junctions using a $\mathrm{Co} 2 \mathrm{FeAl}$ Heusler alloy electrode. Physical Review B 82, 092402 (2010).

120. F. Shen et al., A specular spin valve with discontinuous nano-oxide layers. Applied Physics Letters 80, 4410-4412 (2002).

121. D. L. Zhang, X. G. Xu, Y. Wu, J. Miao, Y. Jiang, Effect of nano-oxide layers on giant magnetoresistance in pseudo-spin-valves using Co2FeAl electrodes. J Magn Magn Mater 323, 631-634 (2011).

122. H. Sakakima, E. Hirota, Y. Kawawake, Enhanced GMR in $[\mathrm{NiFe} / \mathrm{Cu} / \mathrm{Co}]$ with specular reflective capping layers. J Magn Magn Mater 184, 49-54 (1998).

123. W. F. Egelhoff et al., Specular electron scattering in giant magnetoresistance spin valves. IEEE Transactions on Magnetics 33, 3580-3582 (1997).

124. W. F. Egelhoff et al., Oxygen as a surfactant in the growth of giant magnetoresistance spin valves. Journal of Applied Physics 82, 6142-6151 (1997).

125. A. N. Dobrynin et al., Observation of $\mathrm{Co} / \mathrm{CoO}$ nanoparticles below the critical size for exchange bias. Journal of Applied Physics 101, 113913 (2007).

126. M. Gierlings, M. Prandolini, H. Fritzsche, M. Gruyters, D. Riegel, Change and asymmetry of magnetization reversal for a $\mathrm{Co} / \mathrm{CoO}$ exchange-bias system. Physical Review B 65, 092407 (2002).

127. M. Gruyters, D. Riegel, Strong exchange bias by a single layer of independent antiferromagnetic grains: The CoO/Co model system. Physical Review B 63, 052401 (2000). 
128. M. Stiles, R. McMichael, Temperature dependence of exchange bias in polycrystalline ferromagnet-antiferromagnet bilayers. Physical Review B 60, 12950 (1999).

129. A. Khapikov, Theory of the magnetization reversal of ultrathin Fe films on a $\mathrm{Cr}$ substrate. Physical review letters 80, 2209 (1998).

130. B. Jack, P. P. William, Jr., Spin-diffusion lengths in metals and alloys, and spin-flipping at metal/metal interfaces: an experimentalist's critical review. Journal of Physics: Condensed Matter 19, 183201 (2007).

131. A. Manchon, N. Strelkov, A. Deac, A. Vedyayev, B. Dieny, Interpretation of relationship between current perpendicular to plane magnetoresistance and spin torque amplitude. Physical Review B 73, (2006).

132. T. M. Nakatani et al., Bulk and interfacial scatterings in current-perpendicular-to-plane giant magnetoresistance with $\mathrm{Co} 2 \mathrm{Fe}(\mathrm{A} 10.5 \mathrm{Si0} .5)$ Heusler alloy layers and Ag spacer. Applied Physics Letters 96, 212501 (2010).

133. T. Shinjo, Nanomagnetism and spintronics, p.96. (Elsevier, 2013).

134. H. Bremers, J. Hesse, H. Ahlers, J. Sievert, D. Zachmann, Order and magnetic properties of Fe89-xMn11Alx alloys: magnetization measurements and X-ray diffraction. Journal of Alloys and Compounds 366, 67-75 (2004).

135. E. Y. Huang, M. H. Kryder, Fabrication of MnAl thin films with perpendicular anisotropy on Si substrates. Journal of Applied Physics 117, 17E314 (2015).

136. Y. Cui, W. Yin, W. Chen, J. Lu, S. A. Wolf, Epitaxial $\tau$ phase MnAl thin films on $\mathrm{MgO}$ (001) with thickness-dependent magnetic anisotropy. Journal of Applied Physics 110, 103909 (2011).

137. J. H. Park et al., Saturation magnetization and crystalline anisotropy calculations for MnAl permanent magnet. Journal of Applied Physics 107, 09A731 (2010).

138. H. Tanigawa et al., Domain wall resistance in FePt wire with perpendicular magnetic anisotropy. Journal of Applied Physics 99, 08G520 (2006).

139. N. Nagaosa, J. Sinova, S. Onoda, A. H. MacDonald, N. P. Ong, Anomalous Hall effect. Reviews of Modern Physics 82, 1539-1592 (2010).

140. N. Anuniwat, Y. Cui, S. A. Wolf, J. Lu, B. D. Weaver, Recovery of the chemical 
ordering in L10 MnAl epitaxial thin films irradiated by $2 \mathrm{MeV}$ protons. Applied Physics Letters 102, 102406 (2013).

141. A. Milner, A. Gerber, B. Groisman, M. Karpovsky, A. Gladkikh, Spin-Dependent Electronic Transport in Granular Ferromagnets. Physical Review Letters 76, 475-478 (1996).

142. K. Ryu, H. Lee, S. Shin, H. Akinaga, The pinning role of nonferromagnetic $\beta$ phase in the domain wall motion of ferromagnetic MnAs film on GaAs(001). Journal of Applied Physics 103, 07B106 (2008).

143. S. Chikazumi, Physics of magnetism p. 286. Wiley series on the science and technology of materials (Wiley, New York, 1964), pp. xii, 554 p. : ill. ; $523 \mathrm{~cm}$.

144. T. G. S. M. Rijks, S. K. J. Lenczowski, R. Coehoorn, W. J. M. de Jonge, In-plane and out-of-plane anisotropic magnetoresistance in $\$\{\backslash \text { mathrm }\{\mathrm{Ni}\}\}_{-}\{80\}\{\backslash \text { mathrm }\{\mathrm{Fe}\}\}_{-}\{20\} \$$ thin films. Physical Review B 56, 362-366 (1997).

145. L. Berger, Side-Jump Mechanism for the Hall Effect of Ferromagnets. Physical Review B 2, 4559-4566 (1970).

146. C. Sirisathitkul, H. Longkullabutra, P. Kirdtongmee, S. Aslam, J. Gregg, magnetoresistance due to the lorentz force in silicon membrane. Journal of Science and Technology 24, 305 (2002).

147. N. A. Porter, J. C. Gartside, C. H. Marrows, Scattering mechanisms in textured FeGe thin films: Magnetoresistance and the anomalous Hall effect. Physical Review B 90, (2014).

148. A. Hubert, R. Scha $\square$ fer, Magnetic domains: the analysis of magnetic microstructures p. 499. (Springer, Berlin, 2009), pp. xxvi, 686 pages : illustrations (some color); $624 \mathrm{~cm}$.

149. I. Horcas et al., WSXM: A software for scanning probe microscopy and a tool for nanotechnology. Review of Scientific Instruments 78, 013705 (2007).

150. J. C. Slonczewski, Theory of domain-wall motion in magnetic films and platelets. Journal of Applied Physics 44, 1759-1770 (1973).

151. D. Navas, F. Ilievski, C. A. Ross, CoCrPt antidot arrays with perpendicular 
magnetic anisotropy made on anodic alumina templates. Journal of Applied Physics 105, 113921 (2009).

152. M. A. Angadi, V. Thanigaimani, Thickness dependence of the Curie temperature of MnAl films. J Mater Sci Lett 11, 1213-1216 (1992).

153. D. Hinzke et al., Domain wall properties of FePt: From Bloch to linear walls. Physical Review B 77, (2008).

154. C. Tannous, J. Gieraltowski, The Stoner-Wohlfarth model of ferromagnetism. European Journal of Physics 29, 475 (2008).

155. T. Moriyama et al., Magnetic tunnel junctions with L10-ordered FePt alloy electrodes. Journal of applied physics 95, 6789-6791 (2004).

156. C. Zha, S. Bonetti, J. Persson, Y. Zhou, J. Åkerman, Pseudo-spin-valve with L10 (111)-oriented FePt fixed layer. Journal of Applied Physics 105, 07E910 (2009).

157. S. Mitani, K. Tsukamoto, T. Seki, T. Shima, K. Takanashi, Fabrication and characterization of $\mathrm{L} 10$-ordered $\mathrm{FePt} / \mathrm{AlO} / \mathrm{FeCo}$ magnetic tunnel junctions. IEEE transactions on magnetics 41, 2606-2608 (2005).

158. K. M. Seemann et al., Diffusive and ballistic current spin polarization in magnetron-sputtered L 1 0-ordered epitaxial FePt. Physical Review B 76, 174435 (2007).

159. A. Sakuma, First principle calculation of the magnetocrystalline anisotropy energy of FePt and CoPt ordered alloys. Journal of the Physical Society of Japan 63, 3053-3058 (1994).

160. Y. Kurtulus, R. Dronskowski, Electronic structure, chemical bonding, and spin polarization in ferromagnetic MnAl. Journal of Solid State Chemistry 176, 390-399 (2003).

161. L. R. Harriott, Limits of lithography. Proceedings of the IEEE 89, 366-374 (2001).

162. J. Grollier et al., Spin-polarized current induced switching in $\mathrm{Co} / \mathrm{Cu} / \mathrm{Co}$ pillars. Applied Physics Letters 78, 3663-3665 (2001).

163. K. Seshan, Handbook of Thin-Film Deposition Processes and Techniques Principles, Methods, Equipment and Applications (2nd Edition). (William Andrew Publishing/Noyes). 
164. S. K. J. Lenczowski, R. J. M. v. d. Veerdonk, M. A. M. Gijs, J. B. Giesbers, H. H. J. M. Janssen, Current-distribution effects in microstructures for perpendicular magnetoresistance experiments. Journal of Applied Physics 75, 5154-5159 (1994).

165. Y. J. Chuang, F. G. Tseng, W. K. Lin, Reduction of diffraction effect of UV exposure on SU-8 negative thick photoresist by air gap elimination. Microsystem Technologies 8, 308-313 (2002).

166. C. W. Chien et al., Scaling Properties of Step-Etch Perpendicular Magnetic Tunnel Junction With Dual-CoFeB/MgO Interfaces. IEEE Electron Device Letters 35, 738-740 (2014).

167. A. Ruotolo et al., Phase-locking of magnetic vortices mediated by antivortices. Nature Nanotechnology 4, 528-532 (2009).

168. S. Kaka et al., Mutual phase-locking of microwave spin torque nano-oscillators. Nature 437, 389-392 (2005).

169. S. Sani et al., Mutually synchronized bottom-up multi-nanocontact spin-torque oscillators. Nature communications 4, (2013).

170. C. H. Chang, M. H. Kryder, Effect of substrate roughness on microstructure, uniaxial anisotropy, and coercivity of $\mathrm{Co} / \mathrm{Pt}$ multilayer thin films. Journal of Applied Physics 75, 6864-6866 (1994).

171. W. H. Rippard et al., Spin-transfer dynamics in spin valves with out-of-plane magnetized CoNi free layers. Physical Review B 81, 014426 (2010).

172. H. Kubota et al., Spin-torque oscillator based on magnetic tunnel junction with a perpendicularly magnetized free layer and in-plane magnetized polarizer. Applied Physics Express 6, 103003 (2013).

173. G. Hu, T. Thomson, C. T. Rettner, S. Raoux, B. D. Terris, Magnetization reversal in CoPd nanostructures and films. Journal of Applied Physics 97, 10J702 (2005).

174. T. G. Rijks, S. Lenczowski, R. Coehoorn, W. De Jonge, In-plane and out-of-plane anisotropic magnetoresistance in Ni 80 Fe 20 thin films. Physical Review B 56, 362 (1997).

175. K. Bussmann, G. A. Prinz, S.-F. Cheng, D. Wang, Switching of vertical giant magnetoresistance devices by current through the device. Applied Physics Letters 
75, 2476-2478 (1999).

176. A. G. M. Jansen, A. P. v. Gelder, P. Wyder, Point-contact spectroscopy in metals. Journal of Physics C: Solid State Physics 13, 6073 (1980).

177. A. S. Jenkins et al., Spin-torque resonant expulsion of the vortex core for an efficient radiofrequency detection scheme. Nat Nanotechnol 11, 360-364 (2016).

178. M. R. Stan, M. Kabir, S. Wolf, J. Lu, in Proceedings of the 2014 IEEE/ACM International Symposium on Nanoscale Architectures. (ACM, 2014), pp. 37-38.

179. M. Tsoi et al., Excitation of a Magnetic Multilayer by an Electric Current. Physical Review Letters 80, 4281-4284 (1998).

180. W. H. Rippard, M. R. Pufall, S. Kaka, S. E. Russek, T. J. Silva, Direct-Current Induced Dynamics in $\$\{\backslash \text { mathrm }\{\mathrm{C}\} \backslash \text { mathrm }\{\mathrm{o}\}\}_{-}\{90\}\{\backslash \operatorname{mathrm}\{\mathrm{F}\} \backslash \operatorname{mathrm}\{\mathrm{e}\}\}_{-}\{10\} /\{\backslash \operatorname{mathrm}\{\mathrm{N}\}$ $\backslash$ mathrm $\{\mathrm{i}\}\}_{-}\{80\}\{\backslash \text { mathrm }\{\mathrm{F}\} \backslash \mathrm{mathrm}\{\mathrm{e}\}\}_{-}\{20\} \$$ Point Contacts. Physical Review Letters 92, 027201 (2004).

181. D. P. Bernstein et al., Nonuniform switching of the perpendicular magnetization in a spin-torque-driven magnetic nanopillar. Physical Review B 83, 180410 (2011).

182. Y.-T. Cui et al., Single-shot time-domain studies of spin-torque-driven switching in magnetic tunnel junctions. Physical review letters 104, 097201 (2010).

183. J. P. Strachan et al., Direct observation of spin-torque driven magnetization reversal through nonuniform modes. Phys Rev Lett 100, 247201 (2008).

184. S. Yuasa, A. Fukushima, H. Kubota, Y. Suzuki, K. Ando, Giant tunneling magnetoresistance up to $410 \%$ at room temperature in fully epitaxial $\mathrm{Co} / \mathrm{MgO} / \mathrm{Co}$ magnetic tunnel junctions with bcc Co (001) electrodes. Applied Physics Letters 89, (2006).

185. J. Hayakawa, S. Ikeda, Y. M. Lee, F. Matsukura, H. Ohno, Effect of high annealing temperature on giant tunnel magnetoresistance ratio of $\mathrm{CoFeB} / \mathrm{MgO} / \mathrm{CoFeB}$ magnetic tunnel junctions. arXiv preprint cond-mat/0610526, (2006).

186. Z. Zeng et al., High-power coherent microwave emission from magnetic tunnel junction nano-oscillators with perpendicular anisotropy. Acs Nano 6, 6115-6121 (2012). 
187. H.-S. Song et al., Observation of the intrinsic Gilbert damping constant in Co/Ni multilayers independent of the stack number with perpendicular anisotropy. Applied Physics Letters 102, 102401 (2013). 